\title{
Discretização de problemas semilineares dissipativos parabólicos e hiperbólicos em domínios unidimensionais
}

Simone Mazzini Bruschi

Orientador: Prof. Dr. Alexandre Nolasco de Carvalho

Tese apresentada ao Instituto de Ciências Matemáticas e de Computação - ICMC-USP, como parte dos requisitos para obtenção do título de Doutor em Ciências - Área: Matemática. 
$\grave{A}$ minha familia e ao Caio 


\section{Agradecimentos ${ }^{1}$}

Agradeço ao Alexandre pela disponibilidade, pela segurança e paciência com que me orientou neste trabalho.

À minha família e ao Caio pela compreensão, apoio e estímulo que recebi.

Aos meus amigos e às minhas irmãs que tornaram esse período mais agradável.

Aos professores do ICMC-USP que participaram de alguma forma desta etapa e à todos os professores do Departamento de Matemática de Rio Claro - UNESP.

À Beth, à Laura e a Marília pela atenção que nos atenderam durante este período.

À Elisa e à Ana que sempre ajudaram para resolver as questões burocráticas.

Aos funcionários do ICMC-USP e do IGCE-UNESP pela disposição que sempre nos atenderam.

\footnotetext{
${ }^{1}$ Este trabalho teve suporte financeiro parcial da FAPESP e da CAPES
} 


\section{Resumo}

Neste trabalho estudamos a redução do estudo da dinâmica assintótica de problemas de evolução semilineares em espaços de dimensão infinita ao estudo da dinâmica assintótica de problemas de evolução semilineares em espaços de dimensão finita. Mais especificamente, estaremos lidando com os problemas da condução de calor e de ondas.

A forma escolhida para a redução da dimensão é a discretização. Neste sentido, estudamos:

1. A relação entre as dinâmicas assintóticas da equação do calor semilinear em domínios unidimensionais e sua respectira discretização. Mostramos que, para passos suficientemente pequenos, os fluxos sobre os atratores são topologicamente equivalentes, e

2. A relação entre as dinâmicas assintóticas da equação semilinear de ondas amortecidas em domínios unidimensionais e sua respectiva discretização. Neste caso não foi possível obter a equivalência topológica entre os fluxos nos atratores, mas ainda é possível obter relaçōes entre as dinâmicas assintóticas dadas pela semicontinuidade superior e inferior dos atratores. 


\begin{abstract}
In this work we study the reduction of the study of the asymptotic dynamics of semilinear evolution problems in infinite dimensional spaces to the study of the asymptotic dynamics of semilinear evolution problems in finite dimensional spaces. More specifically we will dealing with the heat conduction and wave problems.

The tool choosen for the reduction of dimension is the discretization. In this way, we study:

1. The relationship between the asymptotic dynamics of the semilinear heat equation in one-dimensional domains and its discretization. We prove that for sufficiently small step-size, the flows on the attractors are topologically equivalente, and

2. The relationship between the asymptotic dynamics of semilinear damped wave equations in one-dimensional domains and its discretization. In this case it was not possible to obtain the topological equivalence between the flows in the attractors, however it is still possible to obtain a relationship between the asymptotic dynamics given by the upper and lower semicontinuity of attractors.
\end{abstract}




\section{Índice}

Introduçāo 1

1 Preliminares $\quad 9$

1.1 Introdução . . . . . . . . . . . . . . . . . . . 9

1.2 A equação do calor e sua discretização . . . . . . . . . . . . . . 11

1.3 Problemas de ondas fortemente amortecidas . . . . . . . . . . . . 17

1.3.1 Propriedades espectrais de $C_{\eta} \ldots \ldots \ldots \ldots$

1.3.2 Existência de solução local, global, regularidade e existência de atrator em $Y^{0} \ldots \ldots \ldots \ldots \ldots$

1.4 Discretização do problema de ondas fortemente amortecidas . . . . . . . . 21

1.4.1 Propriedades espectrais de $C_{n n} \ldots \ldots \ldots 22$

1.4.2 Existência de atrator para a discretização do problema de ondas fortemente amortecidas . . . . . . . . . . . . . . . . 24

1.5 Variedades Invariantes . . . . . . . . . . . . . . . . 26

1.6 Semi-continuidade superior . . . . . . . . . . . . . 37

1.7 Teoremas de semi-continuidade inferior . . . . . . . . . . 38

2 Problema Parabólico $\quad 57$

2.1 Discretização . . . . . . . . . . . . . . . . . . . . 59

2.2 O Problema Contínuo . . . . . . . . . . . . . 62

2.3 Convergência Espectral Uniforme . . . . . . . . . . . . . 64

2.3.1 Convergência Uniforme dos Autovalores . . . . . . . . . 65

2.3.2 Convergência Uniforme das Autofunções . . . . . . . . . . . . 66

2.4 Comparação dos Campos Vetoriais . . . . . . . . . . . . . 66 
3 Semi-continuidade superior e inferior de atratores em $\eta$ para o problema contínuo

3.1 Semi-continuidade superior . . . . . . . . . . . . . . 70

3.1.1 Limitação. uniforme em $\eta$ : dos atratores na norma $Y^{0} \ldots \ldots .72$

3.1.2 Limitação. uniforme em $\eta$. dos atratores na norma de $Y^{\prime 1} \ldots \ldots 75$

3.1.3 Semicontinuidade superior dos atratores $\mathcal{A}_{\eta}$ em $\eta=0 \ldots \ldots .79$

3.2 Semi-continuidade inferior dos atratores . . . . . . . . . . . 81

4 Semi-continuidade superior e inferior em $\eta$ para cada $n \quad 89$

4.1 Semi-continuidade superior . . . . . . . . . . . . . . . 90

4.1.1 Limitação uniforme em $n$ e em $\eta$ dos atratores $\mathcal{A}_{\eta n}$ na norma de $Y_{n}^{0} 91$

4.1.2 Limitação uniforme em $n$ e em $\eta$ dos atratores $\mathcal{A}_{\eta n}$ na norma de $Y_{n}^{1} 95$

4.1.3 Semi-continuidade superior dos atratores $\mathcal{A}_{\eta n}$ em $\eta=0$, para cada $n .98$ 4.2 Semi-continuidade inferior . . . . . . . . . . . . . . 100

5 Semi-continuidade superior de atratores dos problemas da onda com $\begin{array}{ll}\text { atrito forte e sua respectiva equação discretizada } & 107\end{array}$

5.1 Comparação dos Semigrupos Lineares . . . . . . . . . . . . . . . 110

5.2 Comparação dos semigrupos não lineares . . . . . . . . . . . . . 118

5.3 Semi-continuidade superior dos atratores $\mathcal{A}_{\eta}$ e $i\left(\mathcal{A}_{\eta n}\right)$ em $H^{1} \times L^{2} \quad \ldots 119$

A Convergência em $n$ e $\eta$ dos autovalores do problema linearizado $\quad 121$ A.1 Algumas estimativas . . . . . . . . . . . . . . . . . . 121

A.2 Convergência de autovalores em $n \ldots \ldots \ldots \ldots \ldots \ldots$

A.3 Convergência de autoralores em $\eta$ e $n \ldots \ldots \ldots \ldots 131$

$\begin{array}{ll}\text { Referências Bibliográficas } & 136\end{array}$ 


\section{Introdução}

Neste trabalho estudaremos dois problemas relacionados à discretização de equações de evolução semilineares, um deles relacionado à equação do calor e o outro relacionado à equação de onda amortecida, ambos em domínio unidimensional. Nos dois casos, nosso estudo se refere à relação entre a dinâmica assintótica dos problemas contínuo e sua respectiva discretizacão.

Assim, nosso trabalho tem por objetivo o estudo das seguintes conjecturas

Conjectura 1. As dinâmicas assintóticas da equação do calor e sua respectiva discretização espacial são "equivalentes".

$\mathrm{e}$

Conjectura 2. As dinâmicas assintóticas da equação da onda com amortecimento e sua respectiva discretização espacial são "equivalentes".

O termo "equivalente" terá significado diferente em cada uma das conjecturas acima e será tornado claro posteriormente.

No que segue passamos a descrever com mais precisão as conjecturas acima e quanto de cada uma fomos capazes de solucionar.

Relativamente a primeira conjectura, consideramos o seguinte problema parabólico escalar

$$
\begin{aligned}
& u_{t}=a u_{x x}+f(u), \quad 0<x<1, t>0 \\
& u_{x}(0)=u_{x}(1)=0, t>0,
\end{aligned}
$$

onde $a>0$ e $f: \mathbb{R} \rightarrow \mathbb{R}$ é uma função de classe $C^{2}$ satisfazendo a seguinte condição de dissipatividade

$$
\limsup _{|u| \rightarrow \infty} f(u) / u<-\delta
$$


para algum $\delta>0$. Também, consideramos a discretização semi-implícita (0.1) com $p$ passos igualmente espaçados

$$
\dot{U}=-a \Delta_{p} U+f(U)
$$

onde $\Delta_{p}$ é a matriz $p \times p$ dada por

$$
\Delta_{p}=p^{2}\left[\begin{array}{ccccccc}
1 & -1 & 0 & \cdots & 0 & 0 & 0 \\
-1 & 2 & -1 & \cdots & 0 & 0 & 0 \\
0 & -1 & 2 & \cdots & 0 & 0 & 0 \\
\vdots & \vdots & \vdots & \ddots & \vdots & \vdots & \vdots \\
0 & 0 & 0 & \cdots & 2 & -1 & 0 \\
0 & 0 & 0 & \cdots & -1 & 2 & -1 \\
0 & 0 & 0 & \cdots & 0 & -1 & 1
\end{array}\right],
$$

$\mathrm{f}(U)=\left(f\left(u_{1}\right), \cdots, f\left(u_{p}\right)\right)^{\top}$ e $U=\left(u_{1}, \cdots, u_{p}\right)^{\top}$.

As hipóteses sobre $f$ garantem a existência de um atrator global $\mathcal{A}$ para (0.1) e um atrator global $\mathcal{A}_{p}$ para (0.3).

Para (0.1) e (0.3), nosso objetivo é mostrar que, se $p$ suficientemente grande, as dinâmicas assintóticas são topologicamente equivalentes, isto é, para passos suficientemente pequenos as dinâmicas assintóticas de (0.1) e (0.3) são topologicamente equivalentes.

Para ilustrar as diferenças que podem ocorrer entre as dinâmicas de (0.1) e (0.3) quando os passos não são suficientemente pequenos, consideramos o caso $p=2 \mathrm{em}(0.3)$; se nós escrevemos, $x_{1}=1 / 4, x_{2}=3 / 4$ e denotamos por $u_{1}(t)=u\left(x_{1}, t\right)$ e $u_{2}(t)=u\left(x_{2}, t\right)$, então temos (já com as condições de fronteira incorporadas) que a equação (0.3) torna-se:

$$
\begin{aligned}
& \dot{u}_{1}=-4 a\left(u_{1}-u_{2}\right)+f\left(u_{1}\right), \\
& \dot{u}_{2}=4 a\left(u_{1}-u_{2}\right)+f\left(u_{2}\right) .
\end{aligned}
$$

Se consideramos $f(u)=u-u^{3}$, observamos que para qualquer valor de $a$ a equação (0.5) tem no máximo nove pontos de equilíbrio enquanto o problema (0.1), para valores pequenos de $a$, pode ter um número arbitrariamente grande de pontos de equilíbrio (ver 
[CI]). Além disso, para $4 a<1 / 3$ nós temos a existência de pontos de equilíbrio para (0.5) os quais são estáveis e da forma que $U=\left(u_{1}, u_{2}\right)$ onde $u_{1} \neq u_{2}$. Se a dinâmica de $(0.5)$ fosse equivalente a dinâmica de $(0.1)$ os pontos de equilíbrio $U$ corresponderiam a pontos de equilíbrio estáveis e não constantes de (0.1); o que é um padrão. É bem conhecido (ver [Ch, $\mathrm{CHo}]$ ) que padrões não existem para o problema (0.1). Mesmo para valores de $a$ não tão pequenos a dinâmica da equação discretizada pode diferir significativamente do problema contínuo. Isto foi mostrado previamente em [Ro]. É claro que um argumento similar poderia ser desenvolvido para valores maiores de $p$. A vantagem de $p=2$ é a possibilidade de calcular todos os pontos de equilíbrio de (0.5) o qual dá um descrição completa do atrator.

Para este problema nós provamos o seguinte resultado comparando as dinấmicas de $(0.1)$ e $(0.3)$.

Teorema 0.1. Para $p$ suficientemente grande, existe um homeomorfismo $H: \mathcal{A} \rightarrow \mathcal{A}_{p} o$ qual leva órbitas em órbitas preservando orientação.

A prova deste resultado requer a imersão do espaço de fase do problema discreto no espaço de fase do problema contínuo. Desde que o problema contínuo é de dimensão infinita, o primeiro passo é reduzi-lo a um problema num espaço de dimensão finita e isto é feito utilizando o Teorema da Variedade Invariante. Infelizmente, se nós consideramos o problema contínuo sobre uma variedade invariante de dimensão finita fixa $n$ e consideramos a discretização com tamanho de passo $n^{-1}$, não somos capazes de provar que os campos de vetores, do problema contínuo e do discreto, são $C^{1}$ próximos (devido ao fato que os autovalores de $-\Delta_{p}$ e os do operador Laplaciano $\mathfrak{H}$ d com condição de Neumann não são uniformemente próximos). Mantendo o problema contínuo sobre uma variedade fixa, a proximidade dos campos de vetores proveniente de $-\Delta_{p}$ e da projeção do Laplaciano na Variedade Invariante ocorre quando o tamanho do passo é muito pequeno e assim a dimensão do problema discreto excede a dimensão da variedade. Nós poderíamos agora projetar o problema discreto sobre uma variedade invariante com a mesma dimensão da variedade do problema contínuo. Fazendo isso, trataríamos da parte dos campos provenientes das projeções dos operadores Laplaciano e de $-\Delta_{p}$, mas isso nos traz complicações pois teríamos que estudar. a convergência das não linearidades projetadas sobre as variedades invariantes. 
Para evitar esta complicação, permitimos que as dimensōes de ambas as variedades invariantes cresçam de tal forma que ambas sejam $C^{1}$ pequenas e portanto muito próximas uma da outra na topologia $C^{1}$. Então, desde que nós já sabemos que os atratores estão contidos em subvariedades de dimensão fixa, nós temos que os campos de vetores são $C^{1}$ próximos nestas subvariedades. A equivalência topológica é consequência da estabilidade estrutural para o problema contínuo.

Existem vários trabalhos na literatura onde esta equivalência topológica está enunciada. Entre eles nós podemos citar [Ha1, FR]. No entanto não existe na literatura nenhuma prova rigorosa de tais resultados.

No que se refere a segunda conjectura, consideramos o seguinte problema escalar hiperbólico

$$
\begin{aligned}
& u_{t t}+2 a u_{t}=u_{x x}+f(u), \quad 0<x<1, t>0 \\
& u_{x}(0)=u_{x}(1)=0, t>0,
\end{aligned}
$$

onde $a>0$ e $f: \mathbb{R} \rightarrow \mathbb{R}$ é uma função de classe $C^{2}$, satisfazendo a condição de dissipatividade (0.2).

Também consideramos a discretização semi-implícita de (0.6) com $n$ passos igualmente espaçados. Denotando $U=\left(u_{1}, \cdots, u_{n}\right)^{\top}$ escrevemos a seguinte equação

$$
\ddot{U}+2 a \dot{U}=-\Delta_{n} U+\mathrm{f}(U)
$$

onde $\Delta_{n}$ é a matriz $n \times n$ dada por $(0.4) \operatorname{com} p=n$ e $\mathrm{f}(U)=\left(f\left(u_{1}\right), \cdots, f\left(u_{n}\right)\right)^{\top}$.

As suposiçōes sobre $f$ garantem a existência de um atrator global, $\mathcal{A}$ para (0.6) e um atrator global $\mathcal{A}_{n}$ para $(0.7)$

Como não há na literatura qualquer resultado garantindo que a propriedade MorseSmale vale genericamente para (0.6), isto, aliado ao fato de não podermos garantir a existência de variedade invariante finito-dimensional faz com que não trabalhemos com equivalência topológica como no caso parabólico. Nosso objetivo, neste caso, será mostrar que as dinâmicas assintóticas são próximas num sentido a ser especificado posteriormente.

Observamos que a equação (0.6) pode ser reescrita como:

$$
\frac{d^{2} u}{d t^{2}}+2 a \frac{d u}{d t}=-A u_{x x}+\frac{\delta}{2} u+f(u)
$$


onde $A: D(A) \subset L^{2} \rightarrow L^{2}$ dado por $A u=-u_{x x}+\frac{\delta}{2} u$. Voltamos a denotar novamente por $f$ a função $f(u)+\frac{\delta}{2} u$.

Reescrevemos a equação (0.6) como um problema abstrato da seguinte forma:

$$
\frac{d}{d t}\left[\begin{array}{l}
u \\
v
\end{array}\right]=C\left[\begin{array}{l}
u \\
v
\end{array}\right]+h\left(\left[\begin{array}{l}
u \\
v
\end{array}\right]\right)
$$

onde

$$
C=\left[\begin{array}{cc}
0 & I \\
-A & -2 a
\end{array}\right], \quad h\left(\left[\begin{array}{l}
u \\
v
\end{array}\right]\right)=\left[\begin{array}{c}
0 \\
f^{e}(u)
\end{array}\right]
$$

e $f^{e}: H^{1} \rightarrow L^{2}$ dada por $f^{e}(u)(x)=f(u(x))$. Observamos que o operador $-C: D(-C) \subset$ $H^{1} \times L^{2} \rightarrow H^{1} \times L^{2}$ não é um operador setorial, pois sobre a reta $\operatorname{Re} \lambda=-a$ há um número infinito de autovalores complexos de $C$ e a parte imaginária desses autovalores não fica limitada. Este fato dificulta a resolução do problema de forma direta pois os resultados utilizados em geral necessitam que o semigrupo de operadores lineares apresente algum tipo de regularização, isto também inviabiliza a utilização do teorema da variedade invariante. Observamos que existem resultados em $[\mathrm{M}]$ que garantem a existência de variedade invariante de dimensão finita quando o amortecimento, ou seja, o parâmetro $a$, é suficientemente grande. Isso nos dá a possibilidade de que a técnica utilizada para o caso parabólico seja empregada neste caso. Como nosso interesse é trabalhar com qualquer valor positivo do parâmetro $a$ e também é sabido que para amortecimento suficientemente pequeno não há existência de variedade invariante de dimensão finita de classe $C^{1}$ (ver [MSM]), nós não abordamos o problema desta forma.

Assim, surgiu a necessidade de trabalharmos com duas outras equaçōes auxiliares as quais teriam boas propriedades. Estas equações auxiliares seriam a equação de onda com amortecimento forte e sua "discretização", e o termo discretização utilizado aqui será explicado oportunamente.

A equação da onda com amortecimento forte, para $0<\theta \leq 1$, é dada por

$$
u_{t t}+2 \eta\left(-\Delta+\frac{\delta}{2}\right)^{\theta} u_{t}+2 a u_{t}=-\left(-\Delta+\frac{\delta}{2}\right) u+f(u),
$$

Reescrevendo o problema (0.11) na forma abstrata temos:

$$
\left.\frac{d}{d t}\left[\begin{array}{l}
u \\
v
\end{array}\right]=C_{\eta, \theta}\left[\begin{array}{l}
u \\
v
\end{array}\right]+h\left(\begin{array}{l}
u \\
v
\end{array}\right]\right)
$$


onde

$$
C_{\eta, \theta}=\left[\begin{array}{cc}
0 & I \\
-A & -2\left(A^{\theta}+a\right)
\end{array}\right]
$$

Por [CT1], temos que o operador $C_{\eta, \theta}$ gera um semigrupo analítico sobre $Y^{0}$ desde que $\theta \geq \frac{1}{2}$, e se $\theta=1$ o resolvente de $C_{\eta, \theta}$ não é compacto. Nossa escolha foi $\theta=\frac{1}{2}$ assim temos que $-C_{\eta, \theta}$ é setorial, tem resolvente compacto e além disso, por [CT2] temos que o domínio de $C_{\eta, \theta}$ é $Y^{1}=X^{1} \times X^{\frac{1}{2}}$ (para outros valores de $\theta$ o domínio envolve uma certa interação entre $u$ e $v$ e não pode ser escrito como um produto cartesiano de potências fracionárias de $X$ ).

Quanto a "discretização", optamos por adotar a discretização do operador $A$, ou seja, $A_{n}=\Delta_{n}+\frac{\delta}{2} I_{n}$ e, desta forma, para $A^{\frac{1}{2}}$, teremos a $A_{n}^{\frac{1}{2}}=\left(\Delta_{n}+\frac{\delta}{2} I_{n}\right)^{\frac{1}{2}}$. Assim, a equação da onda com amortecimento forte discretizada é dada por

$$
\ddot{U}+2 \eta\left(A_{n}\right)^{\frac{1}{2}} \dot{U}+2 a \dot{U}=-A_{n} U+\mathrm{f}(U) .
$$

Reescrevendo o problema (0.14) como um sistema de equações de $1^{a}$ ordem temos:

$$
\frac{d}{d t}\left[\begin{array}{l}
U \\
V
\end{array}\right]=C_{\eta, n}\left[\begin{array}{l}
U \\
V
\end{array}\right]+H\left(\left[\begin{array}{l}
U \\
V
\end{array}\right]\right)
$$

onde

$$
C_{\eta, n}=\left[\begin{array}{cc}
0 & I \\
-A_{n} & -2\left(A_{n}^{\frac{1}{2}}+a\right)
\end{array}\right]
$$

e $H\left(\left[\begin{array}{l}U \\ V\end{array}\right]\right)=\left[\begin{array}{c}0 \\ f(U)\end{array}\right]$

As hipóteses sobre $f$ asseguram a existência de um atrator global $\mathcal{A}_{\eta}$ para $(0.12)$ e um atrator global $\mathcal{A}_{\eta_{n}}$ para $(0.15)$.

Com estas novas equações auxiliares temos os seguintes objetivos:

1. Mostrar a continuidade da família de atratores $\mathcal{A}_{\eta}$ em $\eta=0, \mathcal{A}$.

2. Mostrar a continuidade da família de atratores $\mathcal{A}_{\eta n}$, em $\eta=0, \mathcal{A}_{n}$ uniformemente em $n$, para $n$ suficientemente grande.

3. Mostrar a semi-continuidade superior da família de atratores $\mathcal{A}_{\eta n}$ em $n=\infty$ para cada $\eta>0$. 


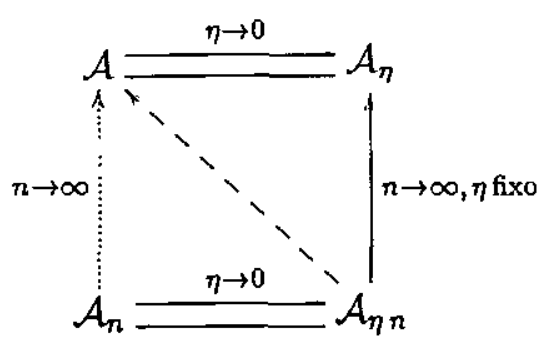

De acordo com o diagrama acima, com estes objetivos alcançados o que nós obteríamos seria a semi-continuidade superior dos atratores $\mathcal{A}_{n}$ em $n=\infty, \mathcal{A}$.

Da mesma forma que o caso parabólico, precisamos considerar em $\mathbb{R}^{n} \times \mathbb{R}^{n}$ normas equivalentes as normas de $Y^{0}$ e $Y^{1}$. Estas normas são dadas de forma análoga ao caso contínuo, através dos operadores $A_{n}$ e $A_{n}^{\frac{1}{2}}$, assim, denotaremos por $Y_{n}^{0}$ e $Y_{n}^{1}$ o espaço $\mathbb{R}^{n} \times \mathbb{R}^{n}$ munido com as respectivas normas de $Y^{0}$ e $Y^{1}$ "discretizadas". Observamos que sempre estamos utilizando o produto interno $L^{2}$ discretizado, induzido pelo produto interno $L^{2}$.

Com relação a semi-continuidade superior em $\eta=0$, tanto para o problema contínuo quanto para o problema discreto, a técnica utilizada é mostrarmos estimativas de limitação uniforme dos atratores nas normas $Y^{0}, Y^{1}$ e $Y_{n}^{0}, Y_{n}^{1}$ respectivamente para os problemas contínuo e discreto. E utilizando imersão compacta e o Teorema de Arzelà-Ascoli obtemos a semi-continuidade superior.

Para a semi-continuidade inferior em $\eta=0$ para os problemas contínuo e discreto utilizamos resultados contidos em [Ha2].

Para a semi-continuidade superior de atratores em $n$ para cada $\eta>0$ fixado, o raciocínio é análogo à [ACR2]. Primeiramente fazemos a comparação dos semigrupos lineares, depois fazemos a comparação dos semigrupos não lineares e finalmente mostramos a semi-continuidade superior dos atratores. Observamos que a limitações uniformes em $\eta$ e $n$ obtidas para os atratores $\mathcal{A}_{\eta n}$ são muito importantes para a comparação dos semigrupos.

Os objetivos 1 e 3 foram alcançados com sucesso. O objetivo 2 foi alcançado parcialmente, pois a semi-continuidade inferior foi obtida apenas para cada $n$ fixado. Como provamos, nos teoremas utilizados para obtermos a semi-continuidade inferior, todas as hipóteses são obtidas de maneira uniforme no parâmetro $n$ e, como observaremos de forma mais profunda no decorrer do trabalho, temos um certo número na demonstração da semi-continuidade inferior que não foi possível obter de maneira uniforme e isto faz 
com que mesmo as hipóteses sendo uniformes não foi possível provar a semi-continuidade inferior de maneira uniforme.

Neste ponto é importante ressaltar que embora não tenhamos mostrado a semi-continuidade superior de $\left\{\mathcal{A}_{n}, \mathcal{A}\right\}$ em $n=\infty$, obtivemos a semi-continuidade superior da família $\left\{\mathcal{A}_{\eta n}, \mathcal{A}\right\}$ em $\eta=0$ e $n=\infty$, o que fornece uma certa redução à dimensão finita do problema $(0.6)$. 


\section{Capítulo 1}

\section{Preliminares}

\subsection{Introdução}

Neste capítulo nós estudaremos alguns resultados básicos necessários ao desenvolvimento do trabalho.

Primeiramente, mostraremos que os problemas semilineares envolvidos e suas respectivas discretizaçōes, geram semigrupos. Desde que estamos interessados em mostrar resultados relativos ao comportamento assintótico destes semigrupos é essencial que estes possuam atratores globais. Assim, o passo seguinte é mostrar que os semigrupos envolvidos possuem propriedades de regularidade e dissipação e consequentemente possuem atratores globais. Algumas estimativas para os atratores dos problemas discretizados já são obtidas aqui independentes do parâmetro $n$ (número de passos).

Quanto ao problema parabólico e sua discretização nosso objetivo é mostrar a equivalência topológica dos problemas contínuo e discreto sobre os atratores e neste caso fazemos uso da Teoria de Variedades Invariantes numa forma adaptada ao resultado que pretendemos. Também utilizamos o fato que o problema parabólico estudado é MorseSmale, genericamente. As definições e os resultados envolvidos estão contidos nas seçōes (1.2) e (1.5).

Quanto ao problema hiperbólico, às equações auxiliares e suas discretizações nosso trabalho está relacionado com a semi-continuidade inferior e superior dos atratores. Neste caso, discutimos os fatos necessários para a obtençāo da semi-continuidade superior. Para a semi-continuidade inferior, enunciamos e provamos resultados que asseguram a sua 
obtenção.

Antes de passarmos a tratar especificamente problemas parabólicos e hiperbólicos semilineares relembraremos alguns conceitos e resultados gerais que serão empregados por todo o texto.

Seja $X$ um espaço de Banach.

Definiçāo 1.1. Uma familia de aplicações $T(t): X \rightarrow X, t \geq 0$, é um $C^{r}$-semigrupo, $r \geq 0$, se:

i) $T(0)=I$;

ii) $T(t+s)=T(t) T(s), t \geq 0, s \geq 0$;

iii) $T(t) x$ é contínuo em $(t, x)$, assim como suas derivadas de Fréchet em relação a $x$ até a ordem $r$ para $(t, x) \in \mathbb{R}^{+} \times X$.

Definição 1.2. Sejam $A$ e $B$ subconjuntos de $X$. Uma distância, $\delta(A, B)$, entre os subconjuntos $A$ e $B$, é dada por

$$
\delta(A, B)=\sup _{y \in A} \inf _{x \in B} d(x, y)
$$

Dizemos que um conjunto $B \subset X$ atrai um conjunto $C \subset X$ sob $T(t)$ se

$$
\delta(T(t) C, B) \rightarrow 0, \quad t \rightarrow \infty
$$

Definição 1.3. Um conjunto $S \subset X$ é invariante se para qualquer $x \in S$ temos que $T(t) x \in S$ para todo $t$.

Definição 1.4. Dizemos que um $C^{r}$-semigrupo, $T(t): X \rightarrow X, t \geq 0$ e $r \geq 0$ é assintoticamente suave se para cada conjunto $B \subset X$, nâo vazio, fechado e limitado para o qual $T(t) B \subset B$, para todo $t \geq 0$, existe um compacto $J \subset B$ que atrai $B$.

Definição 1.5. Um $C^{r}$-semigrupo $T(t): X \rightarrow X: t \geq 0, r \geq 1$ é um sistema gradiente se satisfaz as seguintes condições:

i) Cada órbita positiva é relativamente compacta;

ii) Existe uma função de Lyapunov para $T(t), t \geq 0$, ou seja: existe uma função contínua $V: X \rightarrow \mathbb{R}$ com as seguintes propriedades 
1. $V(x)$ é limitada inferiormente,

2. $V(x) \rightarrow \infty$ quando $|x| \rightarrow \infty$,

3. $V(T(t)) x$ é não crescente em $t$ para cada $x \in X$,

4 Se $T(t) x$ está definido para todo $t \in \mathbb{R}$ e $V(T(t) x)=V(x)$ para $t \in \mathbb{R}$, então $x$ é um ponto de equilibrio.

Definição 1.6. Um conjunto compacto invariante maximal para um $C^{r}$-semigrupo, $T(t)$ é chamado atrator global para $T(t)$ se atrai cada subconjunto limitado de $X$.

Definição 1.7. Um $C^{r}$-semigrupo $T(t): X \rightarrow X, t \geq 0, r>0$ é ponto dissipativo se existe um conjunto $B \subset X$ que atrai cada ponto de $X$ sob $T(t)$.

Teorema 1.1. Se um $C^{r}$-semigrupo $T(t): X \rightarrow X, t \geq 0, r \geq 0$ é assintoticamente suave, ponto dissipativo e órbitas de conjuntos limitados são limitadas, então $T(t), t \geq 0$ possui atrator global.

\subsection{A equação do calor e sua discretização}

Nesta seção mostraremos as propriedades necessárias para o desenvolvimento do trabalho no que se refere à equação do calor e sua discretização.

- Primeiramente, sem perda de generalidade, vamos trabalhar com o parâmetro $a=1$ na equação (0.1). Assim, consideremos a equação do calor

$$
\begin{aligned}
& u_{t}=u_{x x}+f(u), \quad 0<x<1, t>0 \\
& u_{x}(0)=u_{x}(1)=0, t>0
\end{aligned}
$$

onde $f: \mathbb{R} \rightarrow \mathbb{R}$ é uma função de classe $C^{2}$ satisfazendo a seguinte condição de dissipatividade

$$
\limsup _{|u| \rightarrow \infty} f(u) / u<-\delta
$$

para algum $\delta>0$. Reescrevemos a equação (1.2) na forma abstrata dada por

$$
\frac{d u}{d t}=-A u+f^{e} u
$$

onde $A: D(A) \subset X \rightarrow X$ dado por $A u=-u_{x x}$ e $D(A)=\left\{u \in H^{2}(0,1) \mid u^{\prime}(0)=u^{\prime}(1)=\right.$ $0\}: f^{e}: X^{\frac{1}{2}} \rightarrow X^{0}$ dada por $f^{e}(u)(x)=f(u(x))$ e $X=X^{0}=L^{2}(0,1)$ e $X^{\frac{1}{2}}=H^{1}(0,1)$. 
Vamos mostrar que o problema (1.4) gera um semigrupo e este possui atrator global. Temos que $f^{e}$ é localmente lipschitziana sobre subconjuntos limitados de $X^{\frac{1}{2}}$, além disso, $f^{e}$ é de classe $C^{1} \mathrm{e}-A$ é gerador infinitesimal de um $C^{0}$-semigrupo em $X^{0}$, logo dado $u_{0} \in X^{\frac{1}{2}}$ existe uma única solução fraca $u\left(t, u_{0}\right):\left(0, \tau_{0}\right) \rightarrow X^{\frac{1}{2}}$, tal que $u\left(0, u_{0}\right)=u_{0} \mathrm{e}$ $\left(0, \tau_{0}\right)$ é o intervalo maximal de existência.

Agora mostraremos que o intervalo maximal de existência é $(0, \infty)$ para qualquer $u_{0} \in X^{\frac{1}{2}}$. Para isso, mostraremos que $\left\|u\left(t, u_{0}\right)\right\|_{X^{\frac{1}{2}}}<\infty$ para qualquer $t \in\left[0, t_{m}\right)$.

Seja

$$
V(\phi)=\int_{0}^{1} \frac{1}{2}\left|\phi^{\prime}(x)\right|^{2}-\tilde{F}(\phi(x)) d x
$$

onde $\tilde{F}(u)=\int_{0}^{u} f(s) d s$. Pela condição de dissipatividade, temos que existe $c>0$ tal que $\bar{F}(u) \leq-\frac{\delta}{2}|u|^{2}+c$, para todo $u \in \mathbb{R}$. Assim

$$
V(\phi) \geq \frac{1}{2}\left\|\phi^{\prime}\right\|^{2}+\frac{\delta}{2}\|\phi\|^{2}-c \geq m\|\phi\|_{H^{1}}^{2}-c .
$$

Também, se $\|\phi\|_{H^{1}} \leq r$ então

$$
V(\phi) \leq \frac{1}{2}\|\phi\|_{H^{1}}^{2}+K(r) .
$$

Por resultado de regularidade, temos que $u\left(., u_{0}\right) \in C^{1}\left((0, \tau), X^{\frac{1}{2}}\right)$ e $u\left(t, u_{0}\right) \in D(A)$, para $t \in(0, \tau)$ e para todo $u_{0} \in X^{\frac{1}{2}}$. Então,

$$
\frac{d}{d t} V\left(u\left(t, u_{0}\right)\right)=\left\langle A u(t), u^{\prime}(t)\right)-\left\langle f^{e}(u), u^{\prime}(t)\right\rangle=-\left\|u^{\prime}(t)\right\|^{2} \leq 0
$$

Logo: $V\left(u\left(t, u_{0}\right)\right) \leq V\left(u_{0}\right)$, para todo $u_{0} \in X^{\frac{1}{2}}$. Portanto. a solução é limitada em qualquer intervalo $\left[0, t_{m}\right)$.

Assim, temos o seguinte resultado

Teorema 1.2. Definimos $T(t): X^{\frac{1}{2}} \rightarrow X^{\frac{1}{2}}$ dado por $T(t)\left(u_{0}\right)=u\left(t, u_{0}\right)$. Então, $\{T(t)$, $t \geq 0\}$ é um $C^{1}$-semigrupo, assintoticamente suave e é um sistema gradiente. Adicionalmente, o conjunto dos seus pontos de equilibrio é limitado e portanto possui atrator global.

Demonstração: $T(t)$ define um $C^{1}$ semigrupo sobre $X^{\frac{1}{2}}$ pois $f^{e}$ é de classe $C^{1}$. $\mathrm{O}$ operador $A$ é setorial e positivo e o resolvente de $A$ é compacto. Assim, temos que o semigrupo $T(t)$ é compacto e portanto assintoticamente suave. 
Mostraremos agora que o conjunto dos pontos de equilíbrio é limitado em $X^{\frac{1}{2}}$. Seja $\phi$ um ponto de equilíbrio de $T(t)$, então $\phi$ é solução de

$$
\phi^{\prime \prime}+f(\phi)=0
$$

Assim, basta mostrar que $E=\left\{\phi ; \phi^{\prime \prime}+f(\phi)=0\right\}$ é limitado em $H^{1}$. Multiplicando por $\phi$ e integrando por partes, temos $\int_{0}^{1}\left(\phi^{\prime}\right)^{2} d x=\int_{0}^{1} f(\phi) \phi d x$. Pela suposição sobre a $f$ temos que para $\delta / 2$, existe $M>0$ tal que $\frac{f(u)}{u} \leq-\frac{\delta}{2}$ para todo $u$ tal que $|u| \geq M$. Assim, separamos o intervalo $[0,1]$ em $I_{1}=\{x \in[0,1] ;|\phi(x)|<M\}$ e $I_{2}=[0,1] \backslash I_{1}$. Como $f$ é contínua em $\mathbb{R}$ então existe uma constante $K$ tal que $|f(y)| \leq K$ para $|y| \leq M$ e assim temos:

$$
\int_{0}^{1}\left(\phi^{\prime}\right)^{2} d x=\int_{I_{1}} f(\phi) \phi d x+\int_{I_{2}} f(\phi) \phi d x \leq K M-\frac{\delta}{2}\|\phi\|_{L^{2}}
$$

Assim, $\|\phi\|_{H^{1}} \leq K M$. E portanto, $T(t)$ é ponto dissipativo.

Agora mostraremos que $T(t)$ é sistema gradiente. Pela desigualdade (1.6) temos que órbita positiva de conjunto limitado é limitada. Desde que $\overline{\gamma^{+}(\phi)}$ é limitado, fechado e positivamente invariante e, pelo fato que o semigrupo é assintoticamente suave então existe um subconjunto $C \subset \overline{\gamma^{+}(\phi)} \subset X^{\frac{1}{2}}$ que atrai $\overline{\gamma^{+}(\phi)}$. Assim, $\overline{\gamma^{+}(\phi)}$ é pré-compacto. Pela desigualdade (1.5) temos que $V$ é limitada inferiormente e $|V(\phi)| \rightarrow \infty$ quando $\|\phi\| \rightarrow \infty$. Pela desigualdade (1.7) temos que $V$ é não crescente ao longo de órbitas e além disso, se $V(T(t) \phi)=V(\phi)$, então $u_{t}(t, \phi)=0$ logo $u(t, \phi)=\phi$, ou seja, $\phi$ é ponto de equilíbrio.

Consideremos agora

$$
\dot{U}=-\Delta_{p} U+\mathrm{f}(U)
$$

a equação do calor discretizada com $p$ passos igualmente espaçados, onde $\Delta_{p}$ é a matriz 
$p \times p$ dada por

$$
\Delta_{p}=p^{2}\left[\begin{array}{ccccccc}
1 & -1 & 0 & \cdots & 0 & 0 & 0 \\
-1 & 2 & -1 & \cdots & 0 & 0 & 0 \\
0 & -1 & 2 & \cdots & 0 & 0 & 0 \\
\vdots & \vdots & \vdots & \ddots & \vdots & \vdots & \vdots \\
0 & 0 & 0 & \cdots & 2 & -1 & 0 \\
0 & 0 & 0 & \cdots & -1 & 2 & -1 \\
0 & 0 & 0 & \cdots & 0 & -1 & 1
\end{array}\right]
$$

ef $f(U)=\left(f\left(u_{1}\right), \cdots, f\left(u_{p}\right)\right)^{\top}$ e $U=\left(u_{1}, \cdots, u_{p}\right)^{\top}$.

Teorema 1.3. Os autovalores de $\Delta_{p}$ são dados por $\lambda_{k}^{p}=4 p^{2} \operatorname{sen}^{2} \frac{k \pi}{2 p}$ e os áutovetores associados são $w_{k}^{p}=\left(\cos k \pi x_{1}, \cdots, \cos k \pi x_{p}\right)$ para $k=0, \cdots, p-1$.

Além disso, $\lim _{p \rightarrow \infty} \lambda_{k}^{p}=\lambda_{k}$, onde $\lambda_{k}=(k \pi)^{2}$ é $o(k+1)$-ésimo autovetor do operador $-\Delta$ com condição de fronteira homogênea de Neumann. valur

Para obtermos os autovalores e os autovetores de $\Delta_{p}$ procedemos da seguinte forma: sabemos que as autofunções de $-\Delta$ com condição de fronteira de Neumann são dadas por $w_{k}(x)=\cos (k \pi x)$, para $k=0, \cdots$, assim, consideramos os pontos médios $x_{i}$ dos intervalos $\left[\frac{i-1}{p}, \frac{i}{p}\right]$, para $i=1, \cdots, p$ e discretizamos as $p$ primeiras autofunções $w_{k}(x)$ nos pontos $x_{i}$, obtemos $w_{k}^{p}$. Considerando, na definição de autovalor e autovetor, o vetor $w_{k}^{p}$ obtemos como autovalor correspondente $\lambda_{k}^{p}=4 p^{2} \operatorname{sen}^{2} \frac{k \pi}{2 p}$.

Para sermos capazes de comparar a dinâmica do problema discreto com a dinâmica do problema contínuo nós devemos associar a $\mathbb{R}^{p}$ uma norma a qual é compatível com a norma adotada para o problema contínuo. Isto nos conduz a definir em $\mathbb{R}^{p}$ o produto interno: $\langle X, Y\rangle=\frac{1}{p} \sum_{i=1}^{p} x_{i} y_{i}$, o qual é herdado do produto interno $L^{2}$ e será referido como o produto interno $L^{2}$ discretizado. E a norma é dada por $\|U\|^{2}=\left\langle\Delta_{p} U, U\right)+\langle U, U\rangle$, esta norma é a correspondente a norma de $H^{1}$. A norma correspondente a de $L^{2}$ é dada por $\langle U, U\rangle$.

Vamos mostrar que o problema (1.9) gera um semigrupo de classe $C^{2}$ e este possui atrator global. Temos que $f$ é de classe $C^{2} \mathrm{e}-\Delta_{p}$ é gerador infinitesimal de um semigrupo analítico em $\mathbb{R}^{p}$; logo dado $U_{0} \in \mathbb{R}^{p}$ existe uma única solução $U\left(t, U_{0}\right):\left(0, \tau_{0}\right) \rightarrow \mathbb{R}^{p}$, tal que $U\left(0, U_{0}\right)=U_{0}$ e $\left(0, \tau_{0}\right)$ é o intervalo maximal de existência. 
Agora mostraremos que o intervalo maximal de existência é $(0, \infty)$ para qualquer $U_{0} \in \mathbb{R}^{p}$. Para isso, mostraremos que $\left\|U\left(t, U_{0}\right)\right\|<\infty$ para qualquer $t \in\left[0, t_{m}\right)$.

Seja

$$
V(U)=\frac{1}{2}\left\langle\Delta_{p} U, U\right\rangle-\langle\overline{1}, \bar{F}(U)\rangle
$$

onde $\bar{F}(U)=\left(\tilde{F}\left(u_{1}\right), \tilde{F}\left(u_{2}\right), \cdots, \tilde{F}\left(u_{p}\right)\right)$ e $\overline{1}=(1,1, \cdots, 1)$. Como no caso contínuo temos,

$$
V(U) \geq \frac{1}{2}\left\langle\Delta_{p} U, U\right\rangle+\frac{\delta}{2}\langle U, U\rangle-c \geq m\|U\|^{2}-c
$$

Também, para $\|U\| \leq r$ temos

$$
V(U) \leq \frac{1}{2}\|U\|^{2}+K(r)
$$

Temos que, para todo $U_{0}$,

$$
\frac{d}{d t} V\left(U\left(t, U_{0}\right)\right)=\left\langle\Delta_{p} U(t), U^{\prime}(t)\right\rangle-\left\langle\mathrm{f}(U), U^{\prime}(t)\right\rangle=-\left\|U^{\prime}\right\|^{2} \leq 0
$$

Logo: $V\left(U\left(t, U_{0}\right)\right) \leq V\left(U_{0}\right)$, para todo $U_{0} \in \mathbb{R}^{p}$. Portanto, a solução é limitada em qualquer intervalo $\left[0, t_{m}\right)$.

Assim, temos o seguinte resultado

Teorema 1.4. Definimos $T_{p}(t): \mathbb{R}^{p} \rightarrow \mathbb{R}^{p}$ dado por $T_{p}(t)\left(U_{0}\right)=U\left(t, U_{0}\right)$. Então, $\left\{T_{n}(t), t \geq 0\right\}$ é um $C^{2}$-semigrupo, assintoticamente suave e é um sistema gradiente. Adicionalmente, o conjunto dos pontos de seus equilibrio é limitado e portanto possui atrator global.

Demonstração: $T_{p}(t)$ define um $C^{2}$ semigrupo sobre $\mathbb{R}^{p}$. Desde que estamos trabalhando com um espaço de dimensão finita, temos que o semigrupo $T_{p}(t)$ é assintoticamente suave. Mostraremos agora que o conjunto dos pontos de equilíbrio é limitado em $\mathbb{R}^{p}$. Seja $U$ um ponto de equilíbrio de $T_{p}(t)$, então $U^{\prime}(t)=0$ e assim, $U$ é solução de

$$
-\Delta_{p} U+\mathrm{f}(U)=0
$$

Assim, basta mostrar que. $E=\left\{U ;-\Delta_{p} U+\mathrm{f}(U)=0\right\}$ é limitado em $\mathbb{R}^{p}$. Fazendo o produto interno por $U$, temos $\left\langle-\Delta_{p} U, U\right\rangle-\langle\mathrm{f}(U), U\rangle=0$. Pela suposição sobre a $f$ 
temos que para $\delta / 2$, existe $\sigma>0$ tal que $f(u) u \leq-\frac{\delta}{2} u^{2}+\sigma$ para todo $u$. Assim, $\left\langle\Delta_{p} U, U\right\rangle=\langle\mathrm{f}(U), U\rangle \leq-\frac{\delta}{2}\|U\|_{L_{d}^{2}}^{2}+\sigma$ e portanto temos:

$$
\|U\|_{H_{d}^{1}} \leq \sigma m
$$

E portanto, $T_{p}(t)$ é ponto dissipativo.

Agora mostraremos que $T_{p}(t)$ é sistema gradiente. Pela desigualdade (1.12) temos que órbita positiva é limitada. Desde que $\overline{\gamma^{+}(U)}$ é limitado, fechado e positivamente invariante e, pelo fato que o semigrupo é assintoticamente suave então existe um subconjunto $C \subset$ $\overline{\gamma^{+}(U)}$ que atrai $\overline{\gamma^{+}(\bar{U})}$. Assim, $\overline{\gamma^{+}(U)}$ é pré-compacto. Pela desigualdade (1.11) temos que $V$ é limitada inferiormente e $|V(U)| \rightarrow \infty$ quando $\|U\| \rightarrow \infty$. Pela desigualdade ( temos que $V$ é não crescente ao longo de órbitas e além disso, se $V\left(T(t) U_{0}\right)=V\left(U_{0}\right)$, então $\dot{U}\left(t, U_{0}\right)=0$ e portanto $U\left(t, U_{0}\right)=U_{0}$, ou seja, $U_{0}$ é ponto de equilíbrio.

Para o trabalho realizado sobre o problema parabólico também é necessário a propriedade Morse-Smale. Como referência temos [HMO].

Sejam $X, Y$ e $Z$ espaços de Banach.

Definição 1.8. Seja $C^{r}(Y, Z), r \geq 1$ o espaço das aplicações de $Y$ em $Z$ que são limitadas e uniformemente contínuas juntamente com derivadas até ordem r. Para cada $f \in C^{r}(Y, Z)$, seja $T_{f}(t): X \rightarrow X$ um semigrupo fortemente contínuo de transformações de $X$. Para cada $x \in X$, nós supomos que $T_{f}(t) x$ está definido para $t \geq 0$ e é de classe $C^{r}$ em $x$.

Seja

$$
A(f)=\left\{x \in X: T_{f}(t) x \text { está definido e é limitado para } t \in \mathbb{R}\right\}
$$

Temos que se o conjunto $A(f)$ é compacto e todas as órbitas tem fecho compacto então $A(f)$ é um atrator global.

Para $f, g \in C^{r}(Y, Z)$ nós dizemos que $f$ é equivalente a $g, f \sim g$, se existe um homeomorfismo $h: A(f) \rightarrow A(g)$ tal que $h T_{f}=T_{g} h$.

Nós dizemos que $f$ é A-estável se existe uma vizinhança $V$ de $f$ em $C^{r}\left(Y^{*}: Z\right)$ tal que $f \sim g$ para todo $g \in V \cap C^{r}(Y, Z)$.

$O$ conjunto dos pontos não errantes, $\Omega(f)$, de $f$ em $C^{r}(X, X)$ é o conjunto dos pontos $z \in A(f)$ tais que, dada um vizinhança, $U$, de $z$ em $A(f)$ e $T>0$ existe um $t=t(U, T)>$ 
$T$ e $w \in U$ tal que $T_{f}(t)(w) \in U$

Agora estamos em condições de definir Morse-Smale.

Definição 1.9. Um sistema dinâmico $\left\{T_{f}(t), t \geq 0\right\}$ é Morse-Smale se

i. $D T_{f}$ é injetor sobre o espaço tangente de $X$ nos pontos de $A(f)$;

ii. $\Omega(f)$ é a união de um número finito de pontos de equilibrio e órbitas periódicas, todos hiperbólicos com variedades instáveis de dimensão finita;

iii. A variedade estável local e a variedade instável global de todos os pontos de equilibrio e das órbitas periódicas se interceptam transversalmente.

\subsection{Problemas de ondas fortemente amortecidas}

Considere a equação da onda com amortecimento forte

$$
\begin{aligned}
& u_{t t}+2 \eta\left(-\Delta+\frac{\delta}{2}\right)^{1 / 2} u_{t}+2 a u_{t}=u_{x x}+f(u), 0<x<1, t>0 \\
& u_{x}(0)=u_{x}(1)=0, t>0,
\end{aligned}
$$

onde $\eta>0$, $a>0$ e $f: \mathbb{R} \rightarrow \mathbb{R}$ é uma função de classe $C^{2}$, satisfazendo a seguinte condição de dissipatividade (1.3).

Vamos reescrever o problema da seguinte forma:

$$
u_{t t}+2 \eta\left(-\Delta+\frac{\delta}{2}\right)^{1 / 2} u_{t}+2 a u_{t}=-\left(-\Delta+\frac{\delta}{2}\right) u+\frac{\delta}{2} u+f(u) .
$$

Observamos que

$$
\limsup _{|u| \rightarrow \infty} f(u) / u+\frac{\delta}{2}<-\delta / 2 \text {. }
$$

Voltaremos a denotar por $f$ a não linearidade $\frac{\delta}{2} u+f(u)$.

Reescrevendo o problema (1.16) na forma abstrata sobre o espaço $Y^{\prime}=Y^{0}=X^{1 / 2} \times X^{0}$

$$
\begin{aligned}
& \frac{d}{d t}\left[\begin{array}{l}
u \\
v
\end{array}\right]=C_{\eta}\left[\begin{array}{l}
u \\
v
\end{array}\right]+h\left(\left[\begin{array}{l}
u \\
v
\end{array}\right]\right) \\
& {\left[\begin{array}{l}
u \\
v
\end{array}\right]_{t=0}=\left[\begin{array}{l}
u_{0} \\
v_{0}
\end{array}\right]}
\end{aligned}
$$


onde $A=-\Delta+\frac{\delta}{2} I: D(A) \subset X \rightarrow X, D(A)=\left\{u \in H^{2} ; \frac{d u}{d x}(0)=\frac{d u}{d x}(1)=0\right\}, X=L^{2}=$ $X^{0}$,

$$
C_{\eta}=\left[\begin{array}{cc}
0 & I \\
-A & -2\left(\eta A^{1 / 2}+a\right)
\end{array}\right] \text { e } h\left(\left[\begin{array}{l}
u \\
v
\end{array}\right]\right)=\left[\begin{array}{c}
0 \\
f^{e}(u)
\end{array}\right]
$$

e $D\left(C_{\eta}\right)=X^{1} \times X^{\frac{1}{2}}$

Observamos que o operador $A$ é positivo e auto-adjunto, portanto $A^{1 / 2}$ também é. Consideremos o operador $B=2 \eta A^{1 / 2}+B_{1}$ onde $B_{1}=2 a I$. O operador $B$ é auto-adjunto, positivo e satisfaz

$$
\rho_{1} A^{\frac{1}{2}} \leq B \leq \rho_{2} A^{\frac{1}{2}}
$$

De fato, é claro que $B \geq 2 \eta A^{\frac{1}{2}}$ e também, $B \leq\left(2 \eta+2 a \lambda_{1}^{\frac{1}{2}}\right) A^{\frac{1}{2}}$ onde $\lambda_{1}$ é o primeiro autovalor de $A$. Por [CT1], temos que o operador $C_{\eta}$ gera um semigrupo analítico sobre $Y^{0}$ e é de contrações dado que $B$ é dissipativo. De forma mais geral, por [CT2], mesmo no caso onde $B_{1}$ não é tal que $B$ seja comparável com $A^{\frac{1}{2}}$ mas $D\left(A^{\alpha_{1}}\right) \subset D(B)$ para algum $\alpha_{1}<\frac{1}{2}$ então $C_{\eta}$ é gerador de um semigrupo analítico, e para $B_{1}$ dissipativo temos que o semigrupo gerado é de contraçōes. Também, por [CT1], $\beta=-\sup \operatorname{Re} \sigma\left(C_{\eta}\right)>0$, onde $\sigma\left(C_{\eta}\right)$ é o espectro de $C_{\eta}$, e $\left\|e^{C_{\eta} t}\right\| \leq e^{-\beta t}$.

\subsubsection{Propriedades espectrais de $C_{\eta}$}

Sejam $\nu_{k}=(k \pi)^{2}+\frac{\delta}{2}, k=0,1, \cdots$ os autovalores de $A$ então os autovalores, $\lambda_{ \pm k}$, de $C_{\eta}$ são soluções de

$$
\lambda^{2}+\left(2 \eta \nu_{k}^{\frac{1}{2}}+2 a\right) \lambda+\nu_{k}=0 .
$$

e são dados por:

$$
\begin{aligned}
\lambda_{ \pm k} & =-\left(\eta \nu_{k}^{1 / 2}+a\right) \pm \sqrt{\left(\eta \nu_{k}^{1 / 2}+a\right)^{2}-\nu_{k}} \\
& =-\left(\left[\eta+a \nu_{k}^{-1 / 2}\right] \pm \sqrt{\left.\left[\eta+a \nu_{k}^{-1 / 2}\right]^{2}-1\right)} \nu_{k}^{1 / 2}\right.
\end{aligned}
$$

Analisando o sinal de $\left(\eta \nu_{k}^{1 / 2}+a\right)^{2}-\nu_{k}$ obtemos que para cada $\eta>0$ existe um $k_{0}=k_{0}(\eta) \geq 0$ tal que $\lambda_{ \pm k}$ é um número real para $k<k_{0}$ e $\lambda_{ \pm k}$ é um número complexo para $k \geq k_{0}$.

Outro fato é

$$
\lim _{k \rightarrow \infty} \frac{\left|\operatorname{Im}\left(\lambda_{ \pm k}\right)\right|}{\left|\operatorname{Re}\left(\lambda_{ \pm k}\right)\right|}=\frac{\sqrt{1-\eta^{2}}}{\eta}
$$


Assim,

$$
\lim _{\eta \rightarrow 0} \lim _{k \rightarrow \infty} \frac{\left|\operatorname{Im}\left(\lambda_{ \pm k}\right)\right|}{\left|\operatorname{Re}\left(\lambda_{ \pm k}\right)\right|}=\infty
$$

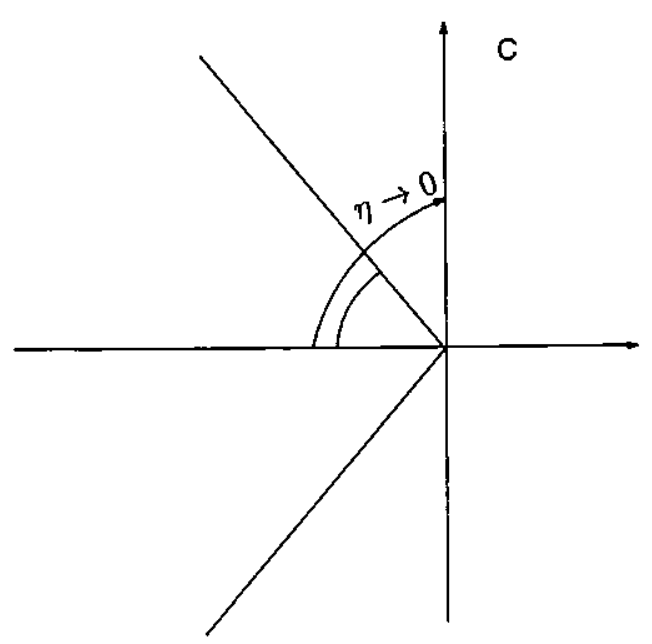

Observamos também que $\lim _{k \rightarrow \infty} \operatorname{Re}\left(\lambda_{ \pm k}\right)=-\infty$

Os correspondentes autovetores são dados por:

$$
\phi_{ \pm k}=\left[\begin{array}{c}
e_{k} \\
\lambda_{ \pm k} e_{k}
\end{array}\right]
$$

onde $e_{k}=\cos (k \pi x)$ é o autovetor de $A$ correspondente ao autovalor $\nu_{k}$. Se $\lambda_{ \pm k}$ é um autovalor duplo então $\psi_{k}=\left[\begin{array}{l}0 \\ e_{k}\end{array}\right]$ é um autovetor generalizado associado a $\lambda_{ \pm k}$. Se $\lambda_{ \pm k}$ é um autovalor complexo então consideraremos os seguintes vetores $\psi_{+k}=\operatorname{Re}\left(\phi_{ \pm k}\right)$ e $\psi_{-k}=$ $\operatorname{Im}\left(\phi_{ \pm k}\right)$, no autoespaço real associado a $\lambda_{ \pm k}$. Então temos as seguintes propriedades:

1) a família $\left(\dot{\phi}_{+k}\right)_{k=0}^{k_{0}},\left(\psi_{+k}\right)_{k=k_{0}}^{\infty}$ é ortogonal sobre $Y^{0}$;

2) a família $\left(\phi_{-k}\right)_{k=0}^{k_{0}},\left(\psi_{-k}\right)_{k=k_{0}}^{\infty}$ é ortogonal sobre $Y^{0}$;

3) $\left\langle\phi_{-i}, \phi_{+j}\right)_{Y^{0}}=0,\left(\psi_{-i}, \psi_{+j}\right\rangle_{Y^{0}}=0,\left(\phi_{-i}, \psi_{+j}\right\rangle_{Y^{0}}=0,\left(\psi_{-i}, \phi_{+j}\right)_{Y^{0}}=0$ se $i \neq j$.

\subsubsection{Existência de solução local, global, regularidade e existên- cia de atrator em $Y^{0}$}

Vamos mostrar que o problema (1.17) gera um semigrupo e este possui atrator global. Temos que $h$ é localmente lipschitziana sobre subconjuntos limitados de $Y^{0}$ e $C_{\eta}$ é gerador infinitesimal de um $C^{0}$-semigrupo em $Y^{0}$, logo dado $\left(u_{0}, v_{0}\right) \in Y^{0}$ existe uma única solu- 
ção fraca $\left[\begin{array}{l}u\left(t, u_{0}, v_{0}\right) \\ v\left(t, u_{0}, v_{0}\right)\end{array}\right]:\left(0, \tau_{0}\right) \rightarrow Y^{0}$, tal que $\left[\begin{array}{l}u\left(0, u_{0}, v_{0}\right) \\ v\left(0, u_{0}, v_{0}\right)\end{array}\right]=\left[\begin{array}{l}u_{0} \\ v_{0}\end{array}\right]$ e $\left(0, \tau_{0}\right)$ é o intervalo maximal de existência.

Agora mostraremos que o intervalo maximal de existência é $(0, \infty)$ para qualquer $\left(u_{0}, v_{0}\right) \in$ $Y^{0}$, para isso, mostraremos que $\left\|\left[\begin{array}{l}u\left(t, u_{0}, v_{0}\right) \\ v\left(t, u_{0}, v_{0}\right)\end{array}\right]\right\|_{Y^{0}}<\infty$ para qualquer $t \in\left[0, t_{m}\right)$.

Seja

$$
V(\phi, \psi)=\frac{1}{2}(A \phi, \phi\rangle+\frac{1}{2}(\psi, \psi\rangle-\int_{0}^{1} \tilde{F}(\phi(x)) d x
$$

onde $\tilde{F}(u)=\int_{0}^{u} f(s) d s$. Pela condição de dissipatividade, temos que existe $c>0$ tal que $\tilde{F}(u) \leq-\frac{\delta}{4}|u|^{2}+c$, para todo $u \in \mathbb{R}$. Assim

$$
V(\phi, \psi) \geq \frac{1}{2}\left(\left\|\phi^{\prime}\right\|^{2}+\frac{\delta}{2}\|\phi\|^{2}\right)+\frac{1}{2}\|\psi\|^{2}+\frac{\delta}{4}\|\phi\|^{2}-c \geq m\left(\|\phi\|_{H^{2}}^{2}+\|\psi\|^{2}\right)-c .
$$

Por um resultado de regularidade, temos que se $\left(u_{0}, v_{0}\right) \in D\left(C_{\eta}\right)$, então $\left[\begin{array}{l}u\left(t, u_{0}, v_{0}\right) \\ v\left(t, u_{0}, v_{0}\right)\end{array}\right] \in$ $C^{1}\left((0, \tau), D\left(C_{\eta}\right)\right)$. Então, para $\left(u_{0}, v_{0}\right) \in D\left(C_{\eta}\right)$ temos

$$
\begin{array}{r}
\frac{d}{d t} V\left(u\left(t, u_{0}, v_{0}\right), v\left(t, u_{0}, v_{0}\right)\right)=(A u(t), v(t)\rangle+\left\langle v_{t}, v\right)+\left\langle f^{e}\langle u), v\right) \\
=\langle A u, v)+\left\langle-2 \eta A^{\frac{1}{2}} v, v\right\rangle-2 a\langle v, v\rangle-\langle A u, v\rangle+\left\langle f^{e}\langle u), v\right\rangle \\
-\left\langle f^{e}(u), v\right)=-2 \eta\left\|A^{1 / 4} v\right\|_{L^{2}}^{2}-2 a\|v\|_{L^{2}}^{2} \leq 0
\end{array}
$$

Logo: $V\left(u\left(t, u_{0}, v_{0}\right), v\left\langle t, u_{0}, v_{0}\right)\right) \leq V\left(u_{0}, v_{0}\right)$. Por densidade de $D\left(C_{\eta}\right)$ em $Y^{0}$ temos que $V\left\langle u\left(t, u_{0}, v_{0}\right) \cdot v\left(t, u_{0}, v_{0}\right)\right) \leq V\left(u_{0}, v_{0}\right)$, para todo $\left(u_{0}, v_{0}\right) \in Y^{0}$.

Assim,

$$
m\left(\left\|\left[\begin{array}{l}
u\left\langle t, u_{0}, v_{0}\right) \\
v\left(t, u_{0}, v_{0}\right)
\end{array}\right]\right\|\right)_{H^{2} \times L^{2}}^{2}-c \leq V\left(u\left(t, u_{0}, v_{0}\right), v\left(t, u_{0}, v_{0}\right)\right) \leq V\left(u_{0}, v_{0}\right) .
$$

Portanto, a solução é limitada em qualquer intervalo $\left[0, t_{m}\right)$.

Assim, temos o seguinte resultado

Teorema 1.5. Definimos $T_{\eta}(t): Y^{0} \rightarrow Y^{0}$ dado por $T_{\eta}(t)\left(u_{0}, v_{0}\right)=\left(u\left(t,\left(u_{0}, v_{0}\right)\right), v(t\right.$, $\left.\left.\left(u_{0}, v_{0}\right)\right)\right)$. Então, $\left\{T_{\eta}(t), t \geq 0\right\}$ é um $C^{1}$-semigrupo, assintoticamente suave e é um sistema gradiente. Adicionalmente, o conjunto dos seus pontos de equilibrio é limitado e portanto, possui atrator global. 
Demonstração: $T_{\eta}(t)$ define um $C^{1}$ semigrupo sobre $Y^{0}$, pois $h$ é de classe $C^{1}$. Por [CT1], o operador $C_{\eta}$ é setorial e positivo e o resolvente de $C_{\eta}$ é compacto. Assim, temos que o semigrupo $T_{\eta}(t)$ é compacto e portanto assintoticamente suave.

Mostraremos agora que o conjunto dos pontos de equilíbrio é limitado em $Y^{0}$. Seja $(\phi, \psi)$ um ponto de equilíbrio de $T_{\eta}(t)$, então $(\phi, \psi)$ é solução de

$$
\begin{aligned}
& \psi=0 \\
& \phi^{\prime \prime}+f(\phi)=0
\end{aligned}
$$

Como já foi mostrado para o problema parabólico, temos que $E=\left\{\phi ; \phi^{\prime \prime}+f(\phi)=0\right\}$ é limitado em $H^{1}$. E assim está provado que os pontos de equilíbrio são limitaḍos na norma de $Y^{0}$.

Agora mostraremos que $T_{\eta}(t)$ é sistema gradiente. Pela desigualdade (1.24) temos que órbita positiva é limitada. Desde que $\overline{\gamma^{+}(\phi, \psi)}$ é limitado, fechado e positivamente invariante e, pelo fato que o semigrupo é assintoticamente suave então existe um subconjunto $C \subset \overline{\gamma^{+}(\phi, \psi)} \subset Y^{0}$ que atrai $\overline{\gamma^{+}(\phi, \psi)}$. Assim, $\overline{\gamma^{+}(\phi, \psi)}$ é pré-compacto. Pela desigualdade (1.20) temos que $V$ é limitada inferiormente e $|V(\phi, \psi)| \rightarrow \infty$ quando $\|(\phi, \psi)\| \rightarrow \infty$. Pela desigualdade (1.23) temos que $V$ é não crescente ao longo de órbitas e além disso, se $V\left(T_{\eta}(t)(\phi, \psi)\right)=V(\phi, \psi)$, entāo $v(t,(\phi, \psi))=u_{t}(t,(\phi, \psi))=0$, logo $u(t,(\phi, \psi))=\phi$ e $\psi=0$ e portanto $T_{\eta}(t)(\phi, \psi)=(\phi, 0)$, ou seja, $(\phi, \psi)$ é ponto de equilíbrio.

\subsection{Discretização do problema de ondas fortemente amortecidas}

No intervalo $[0,1]$ consideramos os pontos $x_{j}=\frac{j-\frac{1}{2}}{n}, j=1, \cdots, n$ e denotamos $u_{j}(t)=$ $u\left(x_{j}, t\right)$. Nós temos, pelo processo de discretização semi-implícito de Euler, que o operador Laplaciano com condição de Neumann $u_{1}=u_{0}, u_{n+1}=u_{n}$ é a matriz $n \times n,-\Delta_{n}$ onde $\Delta_{n}$ é dada por 


$$
\Delta_{n}=n^{2}\left[\begin{array}{ccccccc}
1 & -1 & 0 & \cdots & 0 & 0 & 0 \\
-1 & 2 & -1 & \cdots & 0 & 0 & 0 \\
0 & -1 & 2 & \cdots & 0 & 0 & 0 \\
\vdots & \vdots & \vdots & \ddots & \vdots & \vdots & \vdots \\
0 & 0 & 0 & \cdots & 2 & -1 & 0 \\
0 & 0 & 0 & \cdots & -1 & 2 & -1 \\
0 & 0 & 0 & \cdots & 0 & -1 & 1
\end{array}\right] \text {, }
$$

Denotando $U=\left(u_{1}, \cdots, u_{p}\right)^{\top}$, consideramos a discretização da equação de ondạ fortemente amortecida (1.16) dada por:

$$
\ddot{U}+2 \eta A_{n}^{1 / 2} \dot{U}+2 a \dot{U}=-A_{n} U+\mathrm{f}(U)
$$

onde $A_{n}$ é a matriz $n \times n$ dada por $A_{n}=\Delta_{n}+\frac{\delta}{2} I$ e $\mathrm{f}(U)=\left(f\left(u_{1}\right), \cdots, f\left(u_{p}\right)\right)^{\top}$.

Novamente, colocando em um sistema de equações de $1^{a}$ ordem, obtemos

$$
\begin{aligned}
& \frac{d}{d t}\left[\begin{array}{l}
U \\
V
\end{array}\right]=C_{\eta n}\left[\begin{array}{l}
U \\
V
\end{array}\right]+H\left(\left[\begin{array}{l}
U \\
V
\end{array}\right]\right) \\
& {\left[\begin{array}{l}
U \\
V
\end{array}\right]_{t=0}=\left[\begin{array}{l}
U_{0} \\
V_{0}
\end{array}\right]}
\end{aligned}
$$

onde

$$
C_{\eta n}=\left[\begin{array}{cc}
0 & I_{n} \\
-A_{n} & -2\left(\eta A_{n}^{\mathrm{I} / 2}+a\right)
\end{array}\right] \text { e } H\left(\left[\begin{array}{l}
U \\
V
\end{array}\right]\right)=\left[\begin{array}{c}
0 \\
\mathrm{f}(U)
\end{array}\right]
$$

\subsubsection{Propriedades espectrais de $C_{\eta n}$.}

Os autovalores de $A_{n}$ são dados por $\nu_{k}^{n}=4 n^{2} \sin ^{2} \frac{k \pi}{2 n}+\delta / 2$ e os autovetores associados são $e_{k}^{n}=\left(\cos k \pi x_{1}, \cdots, \cos k \pi x_{n}\right)$ for $k=0, \cdots, n-1$. Assim temos o seguinte teorema:

Teorema 1.6. Os autovalores, $\lambda_{ \pm k}^{n}$, de $C_{\eta n}$ são soluções da equação $\lambda^{2}+\left(2 \eta\left(\nu_{k}^{n}\right)^{\frac{1}{2}}+\right.$ $2 a) \lambda+\nu_{k}^{n}=0$ e são dados por:

$$
\begin{aligned}
\lambda_{ \pm k}^{n} & =-\left(\eta\left(\nu_{k}^{n}\right)^{1 / 2}+a\right) \pm \sqrt{\left(\eta\left(\nu_{k}^{n}\right)^{1 / 2}+a\right)^{2}-\nu_{k}^{n}} \\
& =-\left(\left[\eta+a\left(\nu_{k}^{n}\right)^{-1 / 2}\right] \pm \sqrt{\left[\eta+a\left(\nu_{k}^{n}\right)^{-1 / 2}\right]^{2}-1}\right)\left(\nu_{k}^{n}\right)^{1 / 2}
\end{aligned}
$$


Os correspondentes autovetores são dados por:

$$
\phi_{ \pm k}^{n}=\left[\begin{array}{c}
e_{k}^{n} \\
\lambda_{ \pm k}^{n} e_{k}^{n}
\end{array}\right]
$$

onde $e_{k}^{n}$ é o autovetor de $A_{n}$ correspondente ao autovalor $\nu_{k}^{n}$. Se $\lambda_{ \pm k}^{n}$ é um autovalor duplo então $\psi_{k}^{n}=\left[\begin{array}{c}0 \\ e_{k}^{n}\end{array}\right]$ é um autovetor generalizado associado a $\lambda_{ \pm k}^{n}$

Analisando o sinal de $\left(\eta\left(\nu_{k}^{n}\right)^{1 / 2}+a\right)^{2}-\left(\nu_{k}^{n}\right)$, obtemos que para cada $\eta>0$ e $n>0$ existe um $k_{0}=k_{0}(\eta, n) \geq 0$ tal que $\lambda_{ \pm k}^{n}$ é um número real para $k<k_{0}$ e $\lambda_{ \pm k}^{n}$ é um número complexo para $k_{0}<k<n$.

Como nosso objetivo é comparar os atratores dos problemas (1.17) e (1.27) precisamos associar a $\mathbb{R}^{n} \times \mathbb{R}^{n}$ uma norma compatível com o espaço $Y^{0}$. Assim, definimós em $\mathbb{R}^{n} \times \mathbb{R}^{n}$ o seguinte produto interno:

$$
\left(\left[\begin{array}{l}
U \\
V
\end{array}\right],\left[\begin{array}{c}
W \\
Z
\end{array}\right]\right)=\left\langle A_{n} U, W\right)_{\mathbb{R}^{n}}+\langle V, Z\rangle_{\mathbb{R}^{n}}
$$

onde $\langle U, W\rangle_{\mathbb{R}^{n}}=\sum_{i=1}^{n} \frac{1}{n} u_{i} w_{i}$ o qual é herdado do produto escalar $L^{2}$ e será referido como produto interno $L^{2}$ discretizado. Denotaremos por $Y_{n}^{0}$ o espaço $\mathbb{R}^{n} \times \mathbb{R}^{n}$ com o produto interno dado acima. Também associaremos a $\mathbb{R}^{n} \times \mathbb{R}^{n}$ o produto interno compatível com o produto interno de $Y^{1}$, ou seja,

$$
\left\langle\left[\begin{array}{l}
U \\
V
\end{array}\right],\left[\begin{array}{c}
W \\
Z
\end{array}\right]\right\rangle=\left\langle A_{n} U, A_{n} W\right\rangle_{\mathbb{R}^{n}}+\left\langle A_{n} V, Z\right\rangle_{\mathbb{R}^{n}}
$$

Denotaremos por $Y_{n}^{1}$ o espaço $\mathbb{R}^{n} \times \mathbb{R}^{n}$ com o produto interno dado acima. A distinção entre um produto interno e o outro será feita através do índice referente ao espaço.

Um cálculo simples mostra que

$$
\left\langle A_{n} U, W\right\rangle_{\mathbb{R}^{n}}=\sum_{i=1}^{n-1} n\left(u_{i+1}-u_{i}\right)\left(w_{i+1}-w_{i}\right)+\delta / 2 \sum_{i=1}^{n} \frac{1}{n} u_{i} w_{i}
$$

A norma em $\mathbb{R}^{n} \times \mathbb{R}^{n}$ é definida por $\left\|\left[\begin{array}{l}U \\ V\end{array}\right]\right\|_{\mathbb{P}^{n} \times \mathbb{R}^{n}}=\left\langle\left[\begin{array}{l}U \\ V\end{array}\right],\left[\begin{array}{l}U \\ V\end{array}\right]\right)^{1 / 2}$.

Observamos que chamaremos a $\|U\|_{H_{d}^{1}}=\left\langle\mathcal{A}_{n} U, U\right\rangle_{\mathbb{R}^{n}}^{1 / 2}$ de norma $H^{1}$ discretizada e a $\|U\|_{H_{d}^{2}}=\left\langle A_{n} U, A_{n} U\right\rangle_{\mathbb{R}^{n}}^{1 / 2}$ de norma $H^{2}$ discretizada. Para evitar confusão denotaremos, 
separadamente, por $\left\|U^{\prime}\right\|_{L_{d}^{2}}=\left\langle\Delta_{n} U, U\right\rangle^{\frac{1}{2}}$ a norma $L^{2}$ discretizada da derivada discretizada.

Se $\lambda_{ \pm k}^{n}$ é um autovalor complexo então consideraremos os seguintes vetores $\psi_{+k}^{n}=\operatorname{Re}\left(\phi_{ \pm k}^{n}\right)$ e $\psi_{-k}^{n}=\operatorname{Im}\left(\phi_{ \pm k}^{n}\right)$, no autoespaço real associado a $\lambda_{ \pm k}$. Sobre os autovetores de $C_{\eta n}$ temos as seguintes propriedades:

1) a família $\left(\phi_{+k}^{n}\right)_{k=0}^{k_{0}},\left(\psi_{+k}^{n}\right)_{k=k_{0}}^{n}$ é ortogonal sobre $Y_{n}^{0}$;

2) a família $\left(\phi_{-k}^{n}\right)_{k=0}^{k_{0}},\left(\psi_{-k}^{n}\right)_{k=k_{0}}^{n}$ é ortogonal sobre $Y_{n}^{0}$

3) $\left(\phi_{-i}^{n}, \phi_{+j}^{n}\right\rangle_{Y_{n}^{0}}=0,\left\langle\psi_{-i}^{n}, \psi_{+j}^{n}\right\rangle_{Y_{n}^{0}}=0,\left(\phi_{-i}^{n}, \psi_{+j}^{n}\right\rangle_{Y_{n}^{0}}=0,\left\langle\psi_{-i}^{n}, \phi_{+j}^{n}\right\rangle_{Y_{n}^{0}}=0$ se $i \neq j$.

\subsubsection{Existência de atrator para a discretização do problemá de ondas fortemente amortecidas}

Vamos mostrar que o problema (1.27) gera um semigrupo e este possui atrator global. Temos que $H$ é de classe $C^{2}$ e portanto, localmente lipschitziana sobre subconjuntos limitados de $\mathbb{R}^{n} \times \mathbb{R}^{n}$ e $C_{\eta_{n}}$ é gerador infinitesimal de um $C^{0}$-semigrupo em $\mathbb{R}^{n} \times \mathbb{R}^{n}$, logo dado $\left(U_{0}, V_{0}\right) \in \mathbb{R}^{n} \times \mathbb{R}^{n}$ existe uma única solução $\left[\begin{array}{l}U\left(t, U_{0}, V_{0}\right) \\ V\left(t, U_{0}, V_{0}\right)\end{array}\right]:\left[0, \tau_{0}\right) \rightarrow \mathbb{R}^{n} \times \mathbb{R}^{n}$, tal que $\left[\begin{array}{l}U\left(0, U_{0}, V_{0}\right) \\ V\left(0, U_{0}, V_{0}\right)\end{array}\right]=\left[\begin{array}{l}U_{0} \\ V_{0}\end{array}\right]$ e $\left[0, \tau_{0}\right)$ é o intervalo maximal de existência. Além disso, $\left[\begin{array}{l}U\left(t, U_{0}, V_{0}\right) \\ V\left(t, U_{0}, V_{0}\right)\end{array}\right]$ é de classe $C^{2}$ em $t$ e $\left(U_{0}, V_{0}\right) \in \mathbb{R}^{n} \times \mathbb{R}^{n}$

Agora mostraremos que o intervalo maximal de existência é $[0, \infty)$ para qualquer $\left(U_{0}, V_{0}\right) \in$ $\mathbb{R}^{n} \times \mathbb{R}^{n}$, para isso, mostraremos que $\left\|\left[\begin{array}{l}U\left(t, U_{0}, V_{0}\right) \\ V\left(t, U_{0}, V_{0}\right)\end{array}\right]\right\|<\infty$ para qualquer $t \in\left[0, t_{m}\right)$. Seja

$$
V_{n}(U, V)=\frac{1}{2}\left(A_{n} U, U\right\rangle+\frac{1}{2}(V, V\rangle-\langle\overline{1}, \bar{F}(U)\rangle
$$

onde $\overline{1}=(1,1, \cdots, 1), \bar{F}=\left(\tilde{F}\left(u_{1}\right), \tilde{F}\left(u_{2}\right), \cdots, \tilde{F}\left(u_{1}\right)\right)$ e $\tilde{F}(u)=\int_{0}^{u} f(s) d s$. Como anteriormente, pela condição de dissipatividade, temos que existe $c>0$ tal que $\tilde{F}(u) \leq$ $-\frac{\delta}{4}|u|^{2}+c$, para todo $u \in \mathbb{R}$. Assim

$$
V_{n}(U, V) \geq \frac{1}{2}\left(\left\|U^{\prime}\right\|_{L_{d}^{2}}^{2}+\frac{\delta}{2}\|U\|_{L_{d}^{2}}^{2}\right)+\frac{1}{2}\|V\|_{L_{d}^{2}}^{2}+\frac{\delta}{4}\|U\|_{L_{d}^{2}}^{2}-c \geq m\left(\|U\|_{H_{d}^{1}}^{2}+\|V\|_{L_{d}^{2}}^{2}\right)-c .
$$


Se $\left[\begin{array}{l}U\left(t, U_{0}, V_{0}\right) \\ V\left(t, U_{0}, V_{0}\right)\end{array}\right]$ é solução de $(1.27)$, então para $\left(U_{0}, V_{0}\right) \in \mathbb{R}^{n} \times \mathbb{R}^{n}$ temos

$$
\begin{aligned}
& \frac{d}{d t} V_{n}\left(U\left(t, U_{0}, V_{0}\right), V\left(t, U_{0}, V_{0}\right\rangle\right\rangle=\left\langle A_{n} U(t\rangle, V(t)\right\rangle+\left\langle V_{t}, V\right\rangle+(F(U), V) \\
& =\left\langle A_{n} U, V\right\rangle+\left(-2 \eta A_{n}^{\frac{1}{2}} V, V\right\rangle-2 a\langle V, V\rangle-\left\langle A_{n} U, V\right\rangle+\langle F(U), V\rangle \\
& -\langle F(U), V)=-2 \eta\left\|A_{n}^{1 / 4} V\right\|_{L_{d}^{2}}^{2}-2 a\|V\|_{L_{d}^{2}}^{2} \leq 0
\end{aligned}
$$

Logo: $V_{n}\left(U\left(t, U_{0}, V_{0}\right), V\left(t, U_{0}, V_{0}\right)\right) \leq V_{n}\left(U_{0}, V_{0}\right)$.

Assim,

$$
m\left(\left\|\left[\begin{array}{l}
U\left(t, U_{0}, V_{0}\right) \\
V\left(t, U_{0}, V_{0}\right)
\end{array}\right]\right\|\right)_{\mathbb{R}^{n} \times \mathbb{R}^{n}}^{2}-c \leq V_{n}\left(U\left(t, U_{0}, V_{0}\right), V\left(t, U_{0}, V_{0}\right)\right\rangle \leq V_{n}\left(U_{0}, V_{0}\right)
$$

Portanto, a solução é limitada em qualquer intervalo $\left[0, t_{m}\right)$.

Assim, temos o seguinte resultado

Teorema 1.7. Definimos $T_{\eta n}(t): \mathbb{R}^{n} \times \mathbb{R}^{n} \rightarrow \mathbb{R}^{n} \times \mathbb{R}^{n}$ dado por $T_{\eta n}(t)\left(U_{0}, V_{0}\right)=(U(t$, $\left.\left.\left(U_{0}, V_{0}\right\rangle\right), V\left(t,\left(U_{0}, V_{0}\right)\right)\right)$. Então, $\left\{T_{\eta n}(t), t \geq 0\right\}$ é um $C^{2}$-semigrupo, assintoticamente suave e é um sistema gradiente. Adicionalmente, o conjunto dos seus pontos de equilibrio é limitado, uniformemente em $n$, e portanto possui atrator global.

Demonstração: $T_{\eta n}(t)$ define um $C^{2}$ semigrupo sobre $\mathbb{R}^{n} \times \mathbb{R}^{n}$. Desde que o semigrupo está definido sobre $\mathbb{R}^{n} \times \mathbb{R}^{n}$ que é de dimensão finita, temos que o semigrupo $T_{\eta}(t)$ é assintoticamente suave.

Mostraremos agora que o conjunto dos pontos de equilíbrio é limitado em $\mathbb{R}^{n} \times \mathbb{R}^{n}$. Seja $\left(U_{0}, V_{0}\right)$ um ponto de equilíbrio de $T_{\eta n}(t)$, então $\left(U_{0}, V_{0}\right)$ é solução de

$$
\begin{aligned}
& V_{0}=0 \\
& A_{n} U_{0}+\mathrm{f}\left(U_{0}\right)=0
\end{aligned}
$$

Como, para a equação do calor discretizada, já foi mostrado que $E=\left\{U ; A_{n} U+\mathrm{f}(U)=0\right\}$ é limitado em $\mathbb{R}^{n}$ na norma $H_{d}^{1}$ uniformemente em $n$, então o conjunto dos pontos de equilíbrio é limitado em $\mathbb{R}^{n} \times \mathbb{R}^{n}$ uniformemente em $n$. E portanto, $T_{\eta n}(t)$ é ponto dissipativo.

Agora mostraremos que $T_{\eta n}(t)$ é sistema gradiente. Pela desigualdade (1.34) temos que órbita positiva é limitạda e desde que o espaço é de dimensão finita, é pré-compacto. Pela desigualdade (1.32) temos que $V_{n}$ é limitada inferiormente e $\left.\mid V_{n}(U . V)\right\} \rightarrow \infty$ quando 
$\|(U, V)\| \rightarrow \infty$. Pela desigualdade (1.33) temos que $V_{n}$ é não crescente ao longo de órbitas e além disso, se $V_{n}\left(T_{\eta n}(t)(U, V)=V_{n}(U, V)\right.$, então $V(t,(U, V))=U_{t}(t,(U, V))=0$, logo $U(t,(U, V))=U$ e $V=0$ e portanto $T_{\eta n}(t)(U, V)=(U, 0)$, ou seja, $(U, V)$ é ponto de equilíbrio.

Temos também o seguinte teorema:

Teorema 1.8. $O C^{2}$-semigrupo $\left\{T_{\eta n}, t \geq 0\right\}$ é possui atrator global o qual é limitado independentemente de $n$ e de $\eta$.

Demonstração: Pelo teorema anterior, temos que o semigrupo $T_{\eta n}$ possui atrator global $\mathcal{A}_{\eta n}$ dado por

$$
\mathcal{A}_{\eta n}=W^{u}\left(E_{\eta n}\right)
$$

onde $E_{\eta n}$ é o conjunto dos pontos de equilíbrio de $T_{\eta n}$. Dado $\epsilon>0$ seja $\overline{N_{\epsilon}\left(E_{\eta n}\right)}$. Pelo observado no teorema anterior, se $(U, V) \in \overline{N_{\epsilon}\left(E_{\eta n}\right)}$ então $\|(U, V)\| \leq \sigma+\epsilon$ e $\|U\|_{\infty} \leq$ $\sqrt{2}(\sigma+\epsilon)$. E assim, se $(U, V) \in \overline{N_{\epsilon}\left(E_{\eta n}\right)}$ então

$$
\left|V_{n}(U, V)\right| \leq \frac{1}{2}\left(\|U\|_{H_{d}^{1}}+\|V\|_{L_{d}^{2}}\right)+\max \left\{\left|f(s)\|\| U \|_{L_{d}^{2}} ;\right| s \mid \leq \sqrt{2}(\sigma+\epsilon)\right\}=K\left(\overline{N_{\epsilon}\left(E_{\eta n}\right)}\right)
$$

Observamos que, se $(U, V) \in \mathcal{A}_{\eta_{n}}=W^{u}\left(E_{\eta n}\right)$ então existem $t_{1} \geq 0$ e $\left(U_{0}, V_{0}\right) \in \overline{N_{\epsilon}\left(E_{\eta n}\right)}$ tal que $(U, V)=T_{\eta n}\left(t_{1}\right)\left(U_{0}, V_{0}\right)$. Logo, pelas desigualdades (1.32) e (1.33) temos que

$$
\|(U, V)\|^{2} \leq m-1\left(V_{n}(U, V)+c\right) \leq m^{-1}\left(V_{n}\left(U_{0}, V_{0}\right)+c\right) \leq m^{-1}\left(K\left(\overline{N_{\epsilon}\left(E_{\eta n}\right)}\right)+c\right) .
$$

Desde que as constantes envolvidas não dependem de $\eta$ nem de $n$, o resultado está provado.

\subsection{Variedades Invariantes}

Nesta seção nós enunciamos e provamos um Teorema de Variedade Invariante (1.9) e um lema de Gronwall Generalizado. O teorema é reproduzido do resultado clássico de variedade invariante como em [He2]. A demonstração é adaptada dado que neste teorema é permitido a mudança do espaço (inclusive da sua dimensão) com a mudança do parâmetro e assim também, a dependência da variedade invariante sobre este parâmetro.

Sejam $X$ e $Y$ espaços de Banach, $-A: D(A) \subset X \rightarrow X$ um operador setorial tal que $\operatorname{Re} \sigma(-A)>0$ e $B: D(B) \subset Y \rightarrow Y$ e gerador de um $C^{0}$-grupo de operadores 
lineares limitados $\{S(t), t \geq 0\}$ sobre $Y$. Seja $\{T(t) t \geq 0\}$ o semigrupo analítico de operadores lineares limitados gerado por $A$ e denote por $(-A)^{\alpha}$ a $\alpha$ potência fracionária de $A$ e $X^{\alpha}=D\left((-A)^{\alpha}\right)$ com a norma do gráfico.

Definição 1.10. Sejam $f: X^{\alpha} \times Y^{\alpha} \rightarrow X, g: X^{\alpha} \times Y^{\alpha} \rightarrow Y$ funções contínuas localmente Lipschitz. Um conjunto $S \subset X^{\alpha} \times Y^{\alpha}$ é variedade invariante para uma equação diferencial

$$
\begin{aligned}
& \dot{x}=A x+f(x, y) \\
& \dot{y}=B y+g(x, y),
\end{aligned}
$$

se existe $\sigma: Y^{\alpha} \rightarrow X^{\alpha}$ tal que $S=\left\{(x, y) \in X^{\alpha} \times Y^{\alpha}: x=\sigma(y)\right\}$ e, para qualquer $\left(x_{0}, y_{0}\right) \in S$, existe uma solução $(x(\cdot), y(\cdot))$ da equação diferencial sobre $\mathbb{R}$ tál que $(x(t), y(t)) \in S \forall t \in \mathbb{R}$. Uma variedade invariante $S$ é dita exponencialmente atratora se existem constantes positivas $\gamma$ e $K$ tais que

$$
\|x(t)-\sigma(y(t))\|_{X^{\alpha}} \leq K e^{-\gamma t}\|x(0)-\sigma(y(0))\|_{X^{\alpha}}
$$

sempre que $(x(t), y(t))$ é uma solução da equação differencial.

Teorema 1.9. Sejam $X_{n}, Y_{n}$ uma sequência de espaços de Banach, $A_{n}: D\left(A_{n}\right) \subset X_{n} \rightarrow$ $X_{n}$ uma sequência de operadores setoriais e $B_{n}: D\left(B_{n}\right) \subset Y_{n} \rightarrow Y_{n}$ uma sequência de geradores de $C^{0}$-grupos de operadores lineares limitados. Suponha que $f_{n}: X_{n}^{\alpha} \times Y_{n}^{\alpha} \rightarrow X_{n}$ e $g_{n}: X_{n}^{\alpha} \times Y_{n}^{\alpha} \rightarrow Y_{n}$ são uma sequência de funções satisfazendo:

$$
\begin{aligned}
& \left\|f_{n}(x, y)-f_{n}(z, w)\right\|_{X_{n}} \leq L_{f}\left(\|x-z\|_{X_{n}^{\alpha}}+\|y-w\|_{Y_{n}^{\alpha}}\right) \\
& \left\|f_{n}(x, y)\right\|_{X_{n}} \leq N_{f}
\end{aligned}
$$

para todo $(x, y),(z, w)$ em $X_{n}^{\alpha} \times Y_{n}^{\alpha} e$

$$
\begin{aligned}
& \left\|g_{n}(x, y)-g_{n}(z, w)\right\|_{Y_{n}} \leq L_{g}\left(\|x-z\|_{X_{n}^{\alpha}}+\|y-w\|_{Y_{n}^{\alpha}}\right) \\
& \left\|g_{n}(x, y)\right\|_{Y_{n}} \leq N_{g}
\end{aligned}
$$

para todo $(x, y),(z, w)$ em $X_{n}^{\alpha} \times Y_{n}^{\alpha}$. Assuma que

$$
\begin{aligned}
& \left\|e^{-A_{n} t} w\right\|_{X_{n}^{\alpha}} \leq M_{a} e^{-\beta(n) t}\|w\|_{X_{n}^{\alpha}}, t \geq 0 \\
& \left\|e^{-A_{n} t} w\right\|_{X_{n}^{\alpha}} \leq M_{a} t^{-\alpha} e^{-\beta(n) t}\|w\|_{X_{n}}, \quad t>0, \\
& \left\|e^{-B_{n} t} z\right\|_{Y_{n}^{\alpha}}=\left\|e^{B_{n}(-t)} z\right\|_{Y_{n}^{\alpha}} \leq M_{b} e^{-\rho(n) t} t\|z\| Y_{n}^{\alpha}, t \leq 0, \\
& \left\|e^{-B_{n} t} z\right\|_{Y_{n}^{\alpha}} \leq M_{b}(-t)^{-\alpha} e^{-\rho(n) t}\|z\|_{Y_{n}^{\prime}}, t<0,
\end{aligned}
$$


para qualquer $w \in X_{n}^{\alpha}$ e $z \in Y_{n}^{\alpha}$, onde $\beta(n)-\rho(n) \rightarrow+\infty$ quando $n \rightarrow \infty$. Considere $o$ sistema fracamente acoplado

$$
\left\{\begin{array}{l}
\dot{x}=-A_{n} x+f_{n}(x, y) \\
\dot{y}=-B_{n} y+g_{n}(x, y)
\end{array}\right.
$$

Então, para $n$ suficientemente grande, existe uma variedade invariante exponenciamente atratora para (1.35)

$$
S=\left\{(x, y): x=\sigma_{n}(y), y \in Y_{n}^{\alpha}\right\}
$$

onde $\sigma_{n}: Y_{n}^{\alpha} \rightarrow X_{n}^{\alpha}$ satisfaz

$$
\begin{aligned}
& s(n)=\sup _{\left\{y \in Y_{n}^{\alpha}\right\}}\left\|\sigma_{n}(y)\right\|_{X_{n}^{\alpha}}, \\
& \left\|\sigma_{n}(y)-\sigma_{n}(z)\right\|_{X_{n}^{\alpha}} \leq l(n)\|y-z\|_{Y_{n}^{\alpha}},
\end{aligned}
$$

com $s(n), l(n) \rightarrow 0$ quando $n \rightarrow \infty$. Se $f, g$ são suaves; então, $\sigma_{n}$ é suave e sua derivada $D \sigma_{n}$ satisfaz

$$
\sup _{y \in Y_{n}}\left\|D \sigma_{n}(y)\right\|_{L\left(Y_{n}^{\alpha}, X_{n}^{\alpha}\right)} \leq l(n)
$$

No caso em que nós aplicaremos o teorema da variedade invariante contido no Teorema 1.9, o parâmetro é um número natural $n$. Pois nós separamos o espaço de fase em um espaço gerado pelas $n$ primeiras autofunções do problema e seu complemento ortogonal. Depois projetamos a equação do calor sobre estes espaços e isto produz o par de equações que aparece na afirmação do lema. (Teor mo)

Observamos que o Teorema da Variedade Invariante é utilizado tanto para reduzir o problema a um espaço de dimensão finita, bem como para mostrar a proximidade dos campos vetoriais envolvidos.

Antes de começarmos a demonstração do Teorema 1.9 nós precisamos estabelecer uma versāo generalizada do lema de Gronwall. Isto requer que nós estudemos a convergência das séries

$$
E_{\beta}(z)=\sum_{k=0}^{\infty} \frac{z^{\beta k}}{\Gamma(k \beta+1)}, \quad \tilde{E}_{\beta}(z)=\sum_{k=0}^{\infty} \frac{z^{\beta k}}{\Gamma((k+1) \beta)}
$$

Não é difícil ver que $E_{\beta}$ e $\tilde{E}_{\beta}$ são funções inteiras e seguindo [E] nós podemos obter que existe uma constante $c$ tal que

$$
E_{\beta}(z) \leq c e^{z}
$$


e que dado $d>1$ existe $c^{\prime}>0$ tal que

$$
\tilde{E}_{\beta}(z) \leq c^{\prime} e^{d z}
$$

Lema 1.1 (Lema de Gronwall Generalizado). Sejam $t<r, \phi:[t, r] \rightarrow \mathbb{R}^{+}$uma função contínua, $a:[t, r] \rightarrow \mathbb{R}^{+}$uma função integrável, $b>0$ e $0<\beta \leq 1$. Assuma que

$$
\phi(t) \leq a(t)+b \int_{t}^{\tau}(s-t)^{\beta-1} \phi(s) d s, \quad t \leq r .
$$

Então,

$$
\phi(t) \leq \sum_{k=0}^{\infty}\left(B^{k} a\right)(t)
$$

com

$$
B^{k} a(t)=\int_{t}^{r} \frac{(b \Gamma(\beta))^{k}}{\Gamma(k \beta)}(s-t)^{k \beta-1} a(s) d s .
$$

Além disso, se $a(t) \equiv a=$ const então nós temos que

$$
\phi(t) \leq a E_{\beta}\left((b \Gamma(\beta))^{1 / \beta}(r-t)\right) \leq a c e^{(b \Gamma(\beta))^{1 / \beta}(\tau-t)},
$$

se $a(t)=c_{0} \int_{t}^{\tau}(s-t)^{-\alpha} e^{\rho(s-r)} d s, \rho>0$, então nós temos que

$$
\phi(t) \leq \frac{c_{0}}{b}\left[E_{\beta}\left((b \Gamma(\beta))^{1 / \beta}(r-t)\right)-1\right] \leq \frac{c_{0}}{b}\left[c e^{(b \Gamma(\beta))^{1 / \beta}(r-t)}-1\right]
$$

e finalmente, se $\psi:[t, r] \rightarrow \mathbb{R}^{+}$é uma função contínua e $a(t)=c_{0} \int_{t}^{r}(s-t)^{-\alpha} e^{\rho s} \psi(s) d s$ $\rho>0$ então nós temos que

$$
\phi(t) \leq c_{0} c^{\prime} \Gamma(\beta) \int_{t}^{\tau}(s-t)^{\beta-1} e^{\rho s} e^{d(b \Gamma(\beta))^{1 / \beta}(s-t)} \psi(s) d s .
$$

\section{Demonstraçāo do Lema de Gronwall Generalizado.}

Seja

$$
(B \phi)(t)=b \int_{t}^{r}(s-t)^{\beta-1} \phi(s) d s
$$

Segue de (1.36) que

$$
\begin{aligned}
\phi(t) \leq a(t)+(B \phi)(t) \leq & a(t)+(B a)(t)+\left(B^{2} \phi\right)(t) \\
& \leq \sum_{k=0}^{n-1}\left(B^{k} a\right)(t)+\left(B^{n} \phi\right)(t)
\end{aligned}
$$


Suponha por indução que

$$
\left(B^{n-1} \phi\right)(t)=\int_{t}^{r} \frac{(b \Gamma(\beta))^{n-1}}{\Gamma((n-1) \beta)}(s-t)^{(n-1) \beta-1} \phi(s) d s
$$

então

$$
\begin{aligned}
B\left(B^{n-1} \phi\right)^{(t)} & =b \int_{t}^{r}(s-t)^{\beta-1}\left[\int_{s}^{r} \frac{(b \Gamma(\beta))^{n-1}}{\Gamma((n-1) \beta)}(\theta-s)^{(n-1) \beta-1} \phi(\theta) d \theta\right] d s \\
& =\frac{b^{n} \Gamma(\beta)^{n-1}}{\Gamma((n-1) \beta)} \int_{t}^{r} \phi(\theta)\left[\int_{t}^{\theta}(s-t)^{\beta-1}(\theta-s)^{(n-1) \beta-1} d s\right] d \theta \\
& =\frac{b^{n} \Gamma(\beta)^{n-1}}{\Gamma((n-1) \beta)} \int_{t}^{r} \phi(\theta)\left[\int_{0}^{\theta-t} u^{\beta-1}(\theta-t-u)^{(n-1) \beta-1} d u\right] d \theta
\end{aligned}
$$

Fazendo $z=\frac{u}{\theta-t}$ temos

$$
\begin{aligned}
\left(B^{n} \phi\right) x_{i} & =\frac{b^{n} \Gamma(\beta)^{n-1}}{\Gamma((n-1) \beta)} \int_{t}^{r} \phi(\theta)(\theta-t)^{\beta}\left[\int_{0}^{1} z^{\beta-1}(\theta-t)^{(n-1) \beta-1}(1-z)^{(n-1) \dot{\beta-1}} d z\right] d \theta \\
& =\frac{b^{n} \Gamma(\beta)^{n-1}}{\Gamma((n-1) \beta)} \int_{t}^{r} \phi(\theta)(\theta-t)^{n \beta-1} d \theta \int_{0}^{1} z^{\beta-1}(1-z)^{(n-1) \beta-1} d z
\end{aligned}
$$

Lembrando que

$$
\int_{0}^{1} z^{\beta-1}(1-z)^{(n-1) \beta-1} d z=B(\beta,(n-1) \beta)=\frac{\Gamma(\beta) \Gamma((n-1) \beta)}{\Gamma(n \beta)}
$$

então

$$
\left(B^{n} \phi\right)(t)=\frac{b^{n} \Gamma(\beta)^{n}}{\Gamma(n \beta)} \int_{t}^{\tau} \phi(\theta)(\theta-t)^{n \beta-1} d \theta
$$

Vamos estudar a convergência da série

$$
\sum_{n=0}^{\infty} \frac{z^{n}}{\Gamma(n \beta)}
$$

Então, $a_{n}=\Gamma(n \beta)^{-1}$ e assim,

$$
\frac{a_{n+1}}{a_{n}}=\frac{\Gamma(n \beta)}{\Gamma((n+1) \beta)}=\frac{B(\beta, n \beta)}{\Gamma(\beta)}=\frac{1}{\Gamma(\beta)} \int_{0}^{1} t^{\beta-1}(1-t)^{n \beta-1} d t
$$

Portanto, pelo Teorema da Convegência Dominada de Lebesgue, temos que

$$
\frac{a_{n+1}}{a_{n}} \rightarrow 0
$$

quando $n \rightarrow \infty$.

Segue que $B^{n} \phi(t) \rightarrow 0$ quando $n \rightarrow \infty \mathrm{e}$

$$
\phi(t) \leq \sum_{k=0}^{\infty}\left(B^{k} a\right)(t)
$$


Se $a(t) \equiv a=$ const então

$$
\begin{aligned}
\left(B^{n} a\right)(t) & =\int_{t}^{r} \frac{b^{n} \Gamma(\beta)^{n}}{\Gamma(n \beta)}(s-t)^{n \beta-1} a d s \\
& =\frac{a b^{n} \Gamma(\beta)^{n}}{n \beta \Gamma(n \beta)}(r-t)^{n \beta}=\frac{a b^{n} \Gamma(\beta)^{n}}{\Gamma(n \beta+1)}(r-t)^{n \beta}
\end{aligned}
$$

Segue que

$$
\begin{array}{r}
\phi(t) \leq a E_{\beta}\left((b \Gamma(\beta))^{1 / \beta}(r-t)\right) \\
\leq a c e^{(b \Gamma(\beta))^{1 / \beta}(r-t)} .
\end{array}
$$

Se

$$
a(t)=c_{0} \int_{t}^{r}(s-t)^{-\alpha} e^{\rho(s-r)} d s, \quad \operatorname{com} \alpha=1-\beta
$$

então

$$
\begin{aligned}
\left(B^{n} a\right)(t) & =c_{0} \frac{b^{n} \Gamma(\beta)^{n}}{\Gamma(n \beta)} \int_{t}^{\tau}(s-t)^{n \beta-1}\left[\int_{s}^{\tau}(\theta-s)^{-\alpha} e^{\rho(\theta-r)} d \theta\right] d s \\
& =c_{0} \frac{b^{n} \Gamma(\beta)^{n}}{\Gamma(n \beta)} \int_{t}^{r} e^{\rho(\theta-r)}\left[\int_{t}^{\theta}(s-t)^{n \beta-1}(\theta-s)^{-\alpha} d s\right] d \theta \\
& =c_{0} \frac{b^{n} \Gamma(\beta)^{n}}{\Gamma(n \beta)} \int_{t}^{r} e^{\rho(\theta-r)}\left[\int_{0}^{\theta-t} u^{n \beta-1}(\theta-t-u)^{-\alpha} d u\right] d \theta
\end{aligned}
$$

Fazendo $v=\frac{u}{\theta-t}$ obtemos:

$$
\begin{aligned}
B^{n} a(t) & =c_{0} \frac{b^{n} \Gamma(\beta)^{n}}{\Gamma(n \beta)} \int_{t}^{r} e^{\rho(\theta-r)}\left[\int_{0}^{1} v^{n \beta-1}(\theta-t)^{n \beta-1}(\theta-t)^{1-\alpha}(1-v)^{-1+\beta} d v\right] d \theta \\
& =c_{0} \frac{b^{n} \Gamma(\beta)^{n}}{\Gamma(n \beta)} \int_{t}^{r} e^{\rho(\theta-r)}(\theta-t)^{(n+1) \beta-1} d \theta B(\beta, n \beta) \\
& =c_{0} \frac{b^{n} \Gamma(\beta)^{n+1}}{\Gamma((n+1) \beta)} \int_{t}^{r} e^{\rho(\theta-r)}(\theta-t)^{(n+1) \beta-1} d \theta \\
& \leq c_{0} \frac{b^{n} \Gamma(\beta)^{n+1}}{\Gamma((n+1) \beta)} \int_{0}^{r-t} s^{(n+1) \beta-1} d s
\end{aligned}
$$

Assim,

$$
\begin{aligned}
\sum_{n=0}^{\infty}\left(B^{n} a\right)(t) & \leq c_{0} \sum_{n=0}^{\infty} \frac{b^{n} \Gamma(\beta)^{n+1}}{\Gamma((n+1) \beta)} \int_{0}^{r-t} s^{(n+1) \beta-1} d s \\
& =c_{0} \sum_{n=0}^{\infty} \frac{b^{n} \Gamma(\beta)^{n}(r-t)^{(n+1) \beta}}{\Gamma((n+1) \beta+1)} \phi^{\prime} s \\
& =\frac{c_{0}}{b} \sum_{n=0}^{\infty} \frac{\left(b^{1 / \beta} \Gamma(\beta)^{1 / \beta}(r-t)\right)^{(n+1) \beta}}{\Gamma((n+1) \beta+1)} d / s \\
& =\frac{c_{0}}{b}\left[E_{\beta}\left(b^{1 / \beta} \Gamma(\beta)^{1 / \beta}(r-t)\right)-1\right]
\end{aligned}
$$


Segue que

$$
\begin{aligned}
\phi(t) & \leq \frac{c_{0}}{b}\left[E_{\beta}\left(b^{1 / \beta} \Gamma(\beta)^{1 / \beta}(r-t)\right)-1\right] \\
& \leq \frac{c_{0}}{b}\left[e^{b^{1 / \beta} \Gamma(\beta)^{1 / \beta}(r-t)}-1\right]
\end{aligned}
$$

Se $a(t)=c_{0} \int_{t}^{r}(s-t)^{-\alpha} e^{\rho s} \psi(s) d s$ então

$$
\begin{aligned}
\left(B^{n} a\right)(t) & =c_{0} \int_{t}^{r} \frac{b^{n} \Gamma(\beta)^{n}}{\Gamma(n \beta)}(s-t)^{n \beta-1}\left[\int_{s}^{\mathcal{\tau}}(\theta-s)^{-\alpha} e^{\rho \theta} \psi(\theta) d \theta\right] d s \\
& =c_{0} \int_{t}^{r} \frac{b^{n} \Gamma(\beta)^{n}}{\Gamma(n \beta)} e^{\rho \theta} \psi(\theta)\left[\int_{t}^{\theta}(s-t)^{n \beta-1}(\theta-s)^{-\alpha} d s\right] d \theta \\
& =c_{0} \int_{t}^{r} \frac{b^{n} \Gamma(\beta)^{n}}{\Gamma(n \beta)} e^{\rho \theta} \psi(\theta)(\theta-t)^{(n+1) \beta-1} B(\beta, n \beta) d \theta \\
& =c_{0} \frac{b^{n} \Gamma(\beta)^{n+1}}{\Gamma((n+1) \beta)} \int_{t}^{r} e^{\rho \theta} \psi(\theta)(\theta-t)^{(n+1) \beta-1} d \theta
\end{aligned}
$$

Assim,

$$
\begin{aligned}
\phi(t) & \leq \sum_{n=0}^{\infty}\left(B^{n} a\right)(t)=c_{0} \int_{t}^{\tau} \sum_{n=0}^{\infty} \frac{b^{n} \Gamma(\beta)^{n+1}}{\Gamma((n+1) \beta)} e^{\rho \theta} \psi(\theta)(\theta-t)^{(n+1) \beta-1} d \theta \\
& =c_{0} \Gamma(\beta) \int_{t}^{\tau} \sum_{n=0}^{\infty} \frac{b^{n} \Gamma(\beta)^{n}(\theta-t)^{n \beta}}{\Gamma((n+1) \beta)} e^{\rho \theta} \psi(\theta)(\theta-t)^{\beta-1} d \theta \\
& =c_{0} \Gamma(\beta) \int_{t}^{\tau} \tilde{E}_{\beta}\left((b \Gamma(\beta))^{1 / \beta}(\theta-t)\right) e^{\rho \theta} \psi(\theta)(\theta-t)^{\beta-1} d \theta \\
& \leq c_{0} c^{\prime} \Gamma(\beta) \int_{t}^{\tau} e^{d(b \Gamma(\beta))^{1 / \beta}(\theta-t)} e^{\rho \theta} \psi(\theta)(\theta-t)^{\beta-1} d \theta
\end{aligned}
$$

Demonstração do Teorema 1.9. O primeiro passo é provar a existência da variedade invariante. Dados $D>0$ e $\Delta>0$, seja $\sigma_{n}: Y_{n}^{\alpha} \rightarrow X_{n}^{\alpha}$ satisfazendo

$$
\|\| \sigma_{n}\left\|:=\sup _{y \in Y_{n}^{\circ}}\right\| \sigma_{n}(y)\left\|_{X_{n}^{\alpha}} \leq D, \quad\right\| \sigma_{n}(y)-\sigma_{n}\left(y^{\prime}\right)\left\|_{X_{n}^{a}} \leq \Delta\right\| y-y^{\prime} \|_{Y_{n}^{\alpha}} .
$$

Seja $y(t)=\psi\left(t, \tau, \eta, \sigma_{n}\right)$ a solução de

$$
\frac{d y}{d t}=-B_{n} y+g_{n}\left(\sigma_{n}(y), y\right), \quad \text { for } \quad t<\tau, y(\tau)=\eta,
$$

e defina

$$
G\left(\sigma_{n}\right)(\eta)=\int_{-\infty}^{\tau} e^{-A_{n}(\tau-s)} f_{n}\left(\sigma_{n}(y(s)): y(s)\right) d s
$$


Note que

$$
\left\|G\left(\sigma_{n}\right)(\cdot)\right\|_{X_{n}^{\alpha}} \leq \int_{-\infty}^{\tau} N_{f} M_{a}(\tau-s)^{-\alpha} e^{-\beta(n)(\tau-s)} d s
$$

Seja $n_{0}$ tal que, para $n \geq n_{0},\left\|G\left(\sigma_{n}\right)(\cdot)\right\|_{X_{n}^{\alpha}} \leq D$. A seguir, suponha que $\sigma_{n}$ e $\sigma_{n}^{\prime}$ são funções satisfazendo (1.43), $\eta, \eta^{\prime} \in Y_{n}^{\alpha}$ e denote $y(t)=\psi\left(t, \tau, \eta, \sigma_{n}\right), y^{\prime}(t)=\psi\left(t, \tau, \eta^{\prime}, \sigma_{n}^{\prime}\right)$. Então,

$$
\begin{aligned}
y(t)-y^{\prime}(t) & =e^{-B_{n}(t-\tau)} \eta \\
& +\int_{\tau}^{t} e^{-B_{n}(t-s)} g_{n}\left(\sigma_{n}(y), y\right) d s-e^{-B_{n}(t-\tau)} \eta^{\prime}-\int_{\tau}^{t} e^{-B_{n}(t-s)} g_{n}\left(\sigma_{n}^{\prime}\left(y^{\prime}\right), y^{\prime}\right) d s .
\end{aligned}
$$

E

$$
\begin{aligned}
& \left\|y_{n}(t)-y_{n}^{\prime}(t)\right\|_{Y_{n}^{\alpha}} \leq M_{b} e^{\rho(n)(\tau-t)}\left\|\eta-\eta^{\prime}\right\|_{Y_{n}^{\alpha}} \\
& +M_{b} \int_{t}^{\tau}(s-t)^{-\alpha} e^{\rho(n)(s-t)}\left\|g_{n}\left(\sigma_{n}\left(y_{n}\right), y_{n}\right)-g_{n}\left(\sigma_{n}^{\prime}\left(y_{n}^{\prime}\right), y_{n}^{\prime}\right)\right\|_{Y_{n}} d s \\
& \leq\left. M_{b} e^{\rho(n)(\tau-t)}\left\|\eta-\eta^{\prime}\right\|\right|_{n} ^{\alpha} \\
& +M_{b} L_{g} \int_{t}^{\tau}(s-t)^{-\alpha} e^{-\rho(n)(t-s)}\left(\left\|\sigma_{n}\left(y_{n}\right)-\sigma_{n}^{\prime}\left(y_{n}^{\prime}\right)\right\|_{X_{n}^{\alpha}}+\left\|y_{n}-y_{n}^{\prime}\right\|_{Y_{n}^{\alpha}}\right) d s \\
& \leq M_{b} e^{\rho(n)(\tau-t)}\left\|\eta-\eta^{\prime}\right\|_{Y_{n}^{\alpha}} \\
& +M_{b} L_{g} \int_{t}^{\tau}(s-t)^{-\alpha} e^{\rho(n)(s-t)}\left(\left\|\sigma_{n}\left(y_{n}^{\prime}\right)-\sigma_{n}^{\prime}\left(y_{n}^{\prime}\right)\right\|_{X_{n}^{\alpha}}+(1+\Delta)\left\|y_{n}-y_{n}^{\prime}\right\|_{Y_{n}^{\alpha}}\right) d s \\
& \leq M_{b} e^{\rho(n)(\tau-t)}\left\|\eta-\eta^{\prime}\right\|_{Y_{n}^{\alpha}} \\
& +M_{b} L_{g} \int_{t}^{\tau}(s-t)^{-\alpha} e^{\rho(n)(s-t)}\left((1+\Delta)\left\|y_{n}-y_{n}^{\prime}\right\|_{Y_{n}^{\alpha}}+\left\|\sigma_{n}-\sigma_{n}^{\prime}\right\|_{X_{n}^{\alpha}}\right) d s \\
& \leq M_{b} e^{\rho(n)(\tau-t)}\left\|\eta-\eta^{\prime}\right\|_{Y_{n}^{\alpha}}+M_{b} L_{g}(1+\Delta) \int_{t}^{\tau}(s-t)^{-\alpha} e^{\rho(n)(s-t)}\left\|y_{n}-y_{n}^{\prime}\right\|_{Y_{n}^{\alpha}} d s \\
& +M_{b} L_{g}\left\|\sigma_{n}-\sigma_{n}^{\prime}\right\| \|_{\left.X_{n}^{\alpha}\right)} \int_{t}^{\tau}(s-t)^{-\alpha} e^{\rho(n)(s-t)} d s .
\end{aligned}
$$

Seja

$$
\phi(t)=e^{\rho(n)(t-\tau)}\left\|y_{n}(t)-y_{n}^{\prime}(t)\right\|_{Y_{n}^{\alpha}}
$$

Então,

$$
\begin{aligned}
\phi(t) \leq M_{b}\left[\left\|\eta-\eta^{\prime}\right\|_{Y_{n}^{\alpha}}\right. & +L_{g} \int_{t}^{\tau}(s-t)^{-\alpha} e^{\rho(n)(s-\tau)} d s\left[\left\|\sigma_{n}-\sigma_{n}^{\prime}\right\|_{X_{n}^{\alpha}}\right) \\
& +M_{b} L_{g}(1+\Delta) \int_{t}^{\tau}(s-t)^{-\alpha} \phi(s) d s .
\end{aligned}
$$

Pelo Lema de Gronwall Generalizado

$$
\left\|y_{n}(t)-y_{n}^{\prime}(t)\right\|_{Y_{n}^{\alpha}} \leq\left[c_{1}\left\|\eta-\eta^{\prime}\right\|_{Y_{n}^{\alpha}}+c_{2}\|\| \sigma_{n}-\sigma_{n}^{\prime} \|_{X_{n}^{\alpha}}\right] e^{\left[\rho(n)+c_{r}\right](\tau-t)}
$$


onde $c_{\Gamma}=\left(M_{b} L_{g}(1+\Delta) \Gamma(1-\alpha)\right)^{\frac{1}{1-\alpha}}$.

Assim,

$$
\begin{aligned}
\| G\left(\sigma_{n}\right)(\eta) & -G\left(\sigma_{n}^{\prime}\right)\left(\eta^{\prime}\right) \|_{X_{n}^{\alpha}} \\
& \leq M_{a} \int_{-\infty}^{\tau}(\tau-s)^{-\alpha} e^{-\beta(n)(\tau-s)}\left\|f_{n}\left(\sigma_{n}(y), y\right)-f_{n}\left(\sigma_{n}^{\prime}\left(y^{\prime}\right), y^{\prime}\right)\right\|_{X_{n}} d s \\
& \leq M_{a} \int_{-\infty}^{\tau}(\tau-s)^{-\alpha} e^{-\beta(n)(\tau-s)}\left(L_{f}\left\|\sigma_{n}(y)-\sigma_{n}^{\prime}\left(y^{\prime}\right)\right\|_{X_{n}^{\alpha}}+L_{f}\left\|y-y^{\prime}\right\|_{Y_{n}^{\alpha}}\right) d s \\
& \leq M_{a} \int_{-\infty}^{\tau}(\tau-s)^{-\alpha} e^{-\beta(n)(\tau-s)} L_{f}\left((1+\Delta)\left\|y-y^{\prime}\right\|_{Y_{n}^{\alpha}}+\left\|\sigma_{n}-\sigma_{n}^{\prime}\right\| \|\right) d s \\
& \leq M_{a} \int_{-\infty}^{\tau}(\tau-s)^{-\alpha} e^{-\beta(n)(\tau-s)} L_{f}\left(1+c_{2}(1+\Delta) e^{\left[\rho(n)+c_{\Gamma}\right](\tau-s)}\right) d s\left\|\sigma_{n}-\sigma_{n}^{\prime}\right\| \| \\
& +c_{1} M_{a} L_{f}(1+\Delta) \int_{-\infty}^{\tau}(\tau-s)^{-\alpha} e^{-\left[\beta(n)-\rho(n)-c_{\Gamma}\right](\tau-s)} d s\left\|\eta-\eta^{\prime}\right\|_{Y_{n}^{\alpha} .} .
\end{aligned}
$$

Sejam

$$
I_{\sigma}(n)=M_{a} L_{f} \int_{-\infty}^{\tau}(\tau-s)^{-\alpha} e^{-\beta(n)(\tau-s)}\left(1+c_{2}(1+\Delta) e^{\left[\rho(n)+c_{\Gamma}\right](\tau-s)}\right) d s
$$

e

$$
I_{\eta}(n)=c_{1} M_{a} L_{f}(1+\Delta) \int_{-\infty}^{\tau}(\tau-s)^{-\alpha} e^{-\left[\beta(n)-\rho(n)-c_{\Gamma}\right](\tau-s)} d s .
$$

É fácil ver que, dado $\theta<1$, existe um $n_{0}$ tal que, para $n \geq n_{0}, I_{\sigma}(n) \leq \theta$ e $I_{\eta}(n) \leq \Delta$ e

$$
\left\|G\left(\sigma_{n}\right)(\eta)-G\left(\sigma_{n}^{\prime}\right)\left(\eta^{\prime}\right)\right\|_{X_{n}^{\alpha}} \leq I_{\eta}(n)\left\|\eta-\eta^{\prime}\right\|_{Y_{n}^{o}}+I_{\sigma}(n)\left\|\sigma_{n}-\sigma_{n}^{\prime}\right\| .
$$

As desigualdades (1.46) e (1.47) implicam que $G$ é uma contração da classe de funções que satsiffaz (1.43) nela mesma. Portanto, tem um único ponto fixo $\sigma_{n}^{*}=G\left(\sigma_{n}^{*}\right)$ nesta classe.

$$
\left\{\left(\sigma_{n}^{*}, y\right), y\right) \quad y \in i^{*} ;
$$

Resta provar que $S=\left\{\left(y, \sigma_{n}^{*}(y)\right): y \in Y_{n}^{\alpha}\right\}$ é uma variedade invariante para (1.35). Seja $\left(x_{0}, y_{0}\right) \in S, x_{0}=\sigma_{n}^{*}\left(y_{0}\right)$. Denote por $y_{n}^{*}(t)$ a solução do seguinte problema do valor inicial

$$
\frac{d y}{d t}=-B_{n} y+g_{n}\left(\sigma_{n}^{*}(y), y\right), \quad y(0)=y_{0}
$$

Isto define uma curva $\left(\sigma_{n}^{*}\left(y_{n}^{*}(t)\right), y_{n}^{*}(t)\right) \in S, \quad t \in \mathbb{R}$. Mas a única solução de

$$
\dot{x}=-A_{n} x+f_{n}\left(\sigma_{n}^{*}\left(y_{n}^{*}(t)\right), y_{n}^{*}(t)\right)
$$

que permanece limitada quando $t \rightarrow-\infty$ é

$$
x^{*}(t)=\int_{-\infty}^{t} e^{-A_{n}(t-s)} f_{n}\left(\sigma_{n}^{*}\left(y_{n}^{*}(s)\right) y_{n}^{*}(s)\right) d s=\sigma_{n}^{*}\left(y_{n}^{*}(t)\right) .
$$


Portanto, $\left(\sigma_{n}^{*}\left(y_{n}^{*}(t)\right), y_{n}^{*}(t)\right)$ é uma solução de (1.35) através de $\left(x_{0}, y_{0}\right)$ e a invariância está provada.

De (1.46) é claro que $s(n) \rightarrow 0$ quando $n \rightarrow \infty$ e de (1.47) que $l(n) \rightarrow 0$ quando $n \rightarrow \infty$.

O próximo passo é provar que, para $n$ suficientemente grande, a variedade invariante $S$ é exponencialmente atratora. Especificamente, se $\left(x_{n}(t), y_{n}(t)\right)$ é uma solução de (1.35), existem constantes positivas $\gamma$ e $K$ tais que

$$
\left\|x_{n}(t)-\sigma_{n}^{*}\left(y_{n}(t)\right)\right\|_{X_{n}^{\alpha}} \leq K e^{-\gamma t}\left\|x_{n}\left(t_{0}\right)-\sigma_{n}^{*}\left(y_{n}\left(t_{0}\right)\right)\right\|_{X_{n}^{\alpha}} .
$$

Sejam $\xi(t)=x_{n}(t)-\sigma_{n}^{*}\left(y_{n}(t)\right)$ e $y_{n}^{*}(s, t), s \leq t$ solução de

$$
\begin{aligned}
& \frac{d y_{n}^{*}}{d s}=-B_{n} y_{n}^{*}+g_{n}\left(\sigma_{n}^{*}\left(y_{n}^{*}\right), y_{n}^{*}\right), \quad s \leq t \\
& y_{n}^{*}(t, t)=y_{n}(t), \quad s=t .
\end{aligned}
$$

Entāo,

$$
\begin{aligned}
& \left\|y_{n}^{*}(s, t)-y_{n}(s)\right\|_{Y_{n}^{\alpha}} \\
& =\| e^{-B_{n}(s-t)} y_{n}^{*}(t, t)+\int_{t}^{s} e^{-B_{n}(s-\theta)} g_{n}\left(\sigma_{n}^{*}\left(y_{n}^{*}(\theta, t)\right), y_{n}^{*}(\theta, t)\right) d \theta \\
& -e^{-B_{n}(s-t)} y_{n}(t)-\int_{t}^{s} e^{-B_{n}(\stackrel{\dot{\rho}-\theta}{\theta-s})} g_{n}\left(x_{n}(\theta), y_{n}(\theta)\right) d \theta \|_{Y_{n}^{\alpha}} \\
& \leq M_{b} \int_{s}^{t}(\theta-s)^{-\alpha} e^{\rho(n)(\theta-s)}\left\|g_{n}\left(\sigma_{n}^{*}\left(y_{n}^{*}(\theta, t)\right), y_{n}^{*}(\theta, t)\right)-g_{n}\left(x_{n}(\theta), y_{n}(\theta)\right)\right\|_{Y_{n}} d \theta \\
& \leq M_{b} L_{g} \int_{s}^{t}(\theta-s)^{-\alpha} e^{\rho(n)(\theta-s)}\left(\left\|\sigma_{n}^{*}\left(y_{n}^{*}(\theta, t)\right)-x_{n}(\theta)\right\|_{X_{n}^{\alpha}}+\left\|y_{n}^{*}(\theta, t)-y_{n}(\theta)\right\|_{Y_{n}^{\alpha}}\right) d \theta \\
& \leq M_{b} L_{g} \int_{s}^{t}(\theta-s)^{-\alpha} e^{\rho(n)(\theta-s)}\left(\left\|\sigma_{n}^{*}\left(y_{n}(\theta)\right)-x_{n}(\theta)\right\|_{X_{n}^{\alpha}}+(1+\Delta)\left\|y_{n}^{*}(\theta, t)-y_{n}(\theta)\right\|_{Y_{n}^{\alpha}}\right) d \theta \\
& \leq M_{b} L_{g} \int_{s}^{t}(\theta-s)^{-\alpha} e^{\rho(n)(\theta-s)}\left((1+\Delta)\left\|y_{n}^{*}(\theta, t)-y_{n}(\theta)\right\|_{Y_{n}^{a}}+\|\xi(\theta)\|_{\lambda_{n}^{\alpha}}\right) d \theta \text {. }
\end{aligned}
$$

Portanto,

$$
z(s) \leq M_{b} L_{g}(1+\Delta) \int_{s}^{t}(\theta-s)^{-\alpha} z(\theta) d \theta+M_{b} L_{g} \int_{s}^{t}(\theta-s)^{-\alpha} e^{\rho(n) \theta}\|\xi(\theta)\|_{\lambda_{n}^{\circ}} d \theta
$$

onde $z(s)=e^{\rho(n) s}\left\|y_{n}^{*}(s, t)-y_{n}(t)\right\|_{Y_{n}^{\alpha}}$. Pelo Lema de Gronwall Generalizado,

$$
\left\|y_{n}^{*}(s, t)-y_{n}(s)\right\|_{Y_{n}^{\alpha}} \leq c_{3} \int_{s}^{t}(\theta-s)^{-\alpha} e^{\left[\rho(n)+c_{\Gamma}\right](\theta-s)}\|\xi(\theta)\|_{X_{n}^{\alpha}} d \theta, \quad s \leq t
$$


Seja $s \leq t_{0} \leq t$. No que segue, estimativas para $\left\|y_{n}^{*}(s, t)-y_{n}^{*}\left(s, t_{0}\right)\right\|_{Y_{n}^{\alpha}}$ são obtidas.

$$
\begin{aligned}
\| y_{n}^{*}(s, t) & -y_{n}^{*}\left(s, t_{0}\right)\left\|_{Y_{n}^{\alpha}} \leq\right\| e^{-B_{n}\left(s-t_{0}\right)}\left[y_{n}^{*}\left(t_{0}, t\right)-y_{n}\left(t_{0}\right)\right] \|_{Y_{n}^{\alpha}} \\
& +\left\|\int_{t_{0}}^{s} e^{-B_{n}(s-\theta)}\left[g_{n}\left(\sigma_{n}^{*}\left(y_{n}^{*}(\theta, t)\right), y_{n}^{*}(\theta, t)\right)-g_{n}\left(\sigma_{n}^{*}\left(y_{n}^{*}\left(\theta, t_{0}\right)\right), y_{n}^{*}\left(\theta, t_{0}\right)\right)\right] d \theta\right\|_{Y_{n}^{\alpha}} \\
& \leq c_{3} M_{b} e^{\rho(n)\left(t_{0}-s\right)} \int_{t_{0}}^{t}\left(\theta-t_{0}\right)^{-\alpha} e^{\left[\rho(n)+c_{\Gamma}\right]\left(\theta-t_{0}\right)}\|\xi(\theta)\|_{X_{n}^{\alpha}} d \theta \\
& +M_{b} L_{g}(1+\Delta) \int_{s}^{t_{0}}(\theta-s)^{-\alpha} e^{\rho(n)(\theta-s)}\left\|y_{n}^{*}(\theta, t)-y_{n}^{*}\left(\theta, t_{0}\right)\right\|_{Y_{n}^{\alpha}} d \theta
\end{aligned}
$$

e pelo Lema de Gronwall Generalizado

$$
\left\|y_{n}^{*}(s, t)-y_{n}^{*}\left(s, t_{0}\right)\right\|_{Y_{n}^{\alpha}} \leq c_{4} \int_{t_{0}}^{t}\left(\theta-t_{0}\right)^{-\alpha} e^{\left[\rho(n)+c_{\Gamma}\right](\theta-s)}\|\xi(\theta)\|_{X_{n}^{\alpha}} \cdot d \theta
$$

A seguir, as estimativas acima são utilizadas para estimar $\|\xi(t)\|_{X_{n}^{\alpha}}$.

$$
\begin{aligned}
\xi(t) & -e^{-A_{n}\left(t-t_{0}\right)} \xi\left(t_{0}\right)=x_{n}(t)-\sigma_{n}^{*}\left(y_{n}(t)\right)-e^{-A_{n}\left(t-t_{0}\right)}\left(x_{n}\left(t_{0}\right)-\sigma_{n}^{*}\left(y_{n}\left(t_{0}\right)\right)\right) \\
& =\int_{t_{0}}^{t} e^{-A_{n}(t-s)} f_{n}\left(x_{n}(s), y_{n}(s)\right) d s-\sigma_{n}^{*}\left(y_{n}(t)\right)+e^{-A_{n}\left(t-t_{0}\right)} \sigma_{n}^{*}\left(y_{n}\left(t_{0}\right)\right) \\
& =\int_{t_{0}}^{t} e^{-A_{n}(t-s)} f_{n}\left(x_{n}(s), y_{n}(s)\right) d s-\int_{-\infty}^{t} e^{-A_{n}(t-s)} f_{n}\left(\sigma_{n}^{*}\left(y_{n}^{*}(s, t), y_{n}^{*}(s, t)\right) d s\right. \\
& +e^{-A_{n}\left(t-t_{0}\right)} \int_{-\infty}^{t_{0}} e^{-A_{n}\left(t_{0}-s\right)} f_{n}\left(\sigma_{n}^{*}\left(y_{n}^{*}\left(s, t_{0}\right), y_{n}^{*}\left(s, t_{0}\right)\right) d s\right. \\
& =\int_{t_{0}}^{t} e^{-A_{n}(t-s)}\left[f_{n}\left(x_{n}(s), y_{n}(s)\right)-f_{n}\left(\sigma_{n}^{*}\left(y_{n}^{*}(s, t)\right) y_{n}^{*}(s, t)\right)\right] d s \\
& -\int_{-\infty}^{t_{0}} e^{-A_{n}(t-s)}\left[f_{n}\left(\sigma_{n}^{*}\left(y_{n}^{*}(s, t)\right), y_{n}^{*}(s, t)\right)-f_{n}\left(\sigma_{n}^{*}\left(y_{n}^{*}\left(s, t_{0}\right)\right) y_{n}^{*}\left(s, t_{0}\right)\right)\right] d s
\end{aligned}
$$




$$
\begin{aligned}
z(t) & =\left\|\xi(t)-e^{-A_{n}\left(t-t_{0}\right)} \xi\left(t_{0}\right)\right\|_{X_{n}^{\alpha}} \\
& \leq M_{a} L_{f} \int_{t_{0}}^{t}(t-s)^{-\alpha} e^{-\beta(n)(t-s)}\left(\left\|x_{n}(s)-\sigma_{n}^{*}\left(y_{n}^{*}(s, t)\right)\right\|_{X_{n}^{\alpha}}+\left\|y_{n}(s)-y_{n}^{*}(s, t)\right\|_{Y_{n}^{\alpha}}\right) d s \\
& +M_{a} L_{f}(1+\Delta) \int_{-\infty}^{t_{0}}(t-s)^{-\alpha} e^{-\beta(n)(t-s)}\left\|y_{n}^{*}\left(s, t_{0}\right)-y_{n}^{*}(s, t)\right\|_{Y_{n}^{\alpha}} d s \\
& \leq M_{a} L_{f} \int_{t_{0}}^{t}(t-s)^{-\alpha} e^{-\beta(n)(t-s)}\|\xi(s)\|_{X_{n}^{\alpha}} d s \\
& +c_{3} M_{a} L_{f}(1+\Delta) \int_{t_{0}}^{t}(t-s)^{-\alpha} e^{-\beta(n)(t-s)} \int_{s}^{t}(\theta-s)^{-\alpha} e^{\left[\rho(n)+c_{\Gamma}\right](\theta-s)}\|\xi(\theta)\|_{X_{n}^{\alpha}} d \theta d s \\
& +c_{4} M_{a} L_{f}(1+\Delta) \int_{-\infty}^{t t_{0}}(t-s)^{-\alpha} e^{-\beta(n)(t-s)} \int_{t_{0}}^{t}\left(\theta-t_{0}\right)^{-\alpha} e^{\left[\rho(n)+c_{\Gamma}\right](\theta-s)}\|\xi(\theta)\|_{X_{n}^{\alpha}} d \theta d s \\
& \leq M_{a} L_{f} \int_{t_{0}}^{t}(t-s)^{-\alpha} e^{-\beta(n)(t-s)}\|\xi(s)\|_{X_{n}^{\alpha}} d s \\
& +c_{5} \int_{t_{0}}^{t}(t-\theta)^{-\alpha} e^{-\beta(n)(t-\theta)}\|\xi(\theta)\|_{X_{n}^{\alpha}} \int_{t_{0}}^{\theta}(\theta-s)^{-\alpha} e^{-\left[\beta(n)-\left(\rho(n)+c_{\Gamma}\right)\right](\theta-s)} d s d \theta \\
& +c_{6} \int_{t_{0}}^{t}\left(\theta-t_{0}\right)^{-\alpha} e^{\left[\rho(n)+c_{\Gamma}\right](\theta-t)}\|\xi(\theta)\|_{X_{n}^{\alpha}} \int_{-\infty}^{t}(t-s)^{-\alpha} e^{-\left[\beta(n)-\left[\rho(n)+c_{\Gamma}\right]\right](t-s)} d s d \theta \\
& \leq\left[M_{a} L_{f}+\frac{c_{5} \Gamma(1-\alpha)}{\left[\beta(n)-\rho(n)-c_{\Gamma}\right]^{1-\alpha}}\right] \int_{t_{0}}^{t}(t-s)^{-\alpha} e^{-\beta(n)(t-s)}\|\xi(s)\|_{X_{n}^{\alpha} d s} d s \\
& +\frac{c_{6} \Gamma(1-\alpha)}{\left[\beta(n)-\rho(n)-c_{\Gamma}\right]^{1-\alpha}} \int_{t_{0}}^{t}\left(\theta-t_{0}\right)^{-\alpha} e^{\left[\rho(n)+c_{\Gamma}\right](\theta-t)}\|\xi(\theta)\|_{X_{n}^{\alpha}} d \theta
\end{aligned}
$$

Assim,

$$
\begin{aligned}
& \|\xi(t)\|_{X_{n}^{\alpha}} \leq M_{\alpha} e^{-\beta(n)\left(t-t_{0}\right)}\left\|\xi\left(t_{0}\right)\right\|_{X_{n}^{\alpha}} \\
& +\left[M_{\alpha} L_{f}+\frac{c_{5} \Gamma(1-\alpha)}{\left[\beta(n)-\rho(n)-c_{\Gamma}\right]^{1-\alpha}}\right] \int_{t_{0}}^{t}(t-s)^{-\alpha} e^{-\beta(n)(t-s)}\|\xi(s)\|_{X_{n}^{\alpha}} d s \\
& +\frac{c_{6} \Gamma(1-\alpha)}{\left[\overline{\left.\beta(n)-\rho(n)-c_{\Gamma}\right]^{1-\alpha}}\right.} \int_{t_{0}}^{t}\left(s-t_{0}\right)^{-\alpha} e^{\left[\rho(n)+c_{\Gamma}\right](s-t)}\|\xi(s)\|_{X_{n}^{\alpha}} d s
\end{aligned}
$$

e, se $w(t)=\sup \left\{\|\xi(s)\|_{X_{n}^{\alpha}}, t_{0} \leq s \leq t\right\}$, então

$$
e^{\beta(n)\left(t-t_{0}\right)}\|\xi(t)\|_{X_{n}^{\alpha}} \leq M_{a}\left\|\xi\left(t_{0}\right)\right\|_{X_{n}^{\circ}}+\gamma(n) e^{\beta(n)\left(t-t_{0}\right)} w(t)
$$

onde

$$
\gamma(n)=\frac{\Gamma(1-\alpha)}{\beta(n)^{1-\alpha}}\left[M_{a} L_{f}+\frac{c_{5} \Gamma(1-\alpha)}{\left[\beta(n)-\rho(n)-c_{\Gamma}\right]^{1-\alpha}}\right]+\frac{c_{6} \Gamma(1-\alpha) K}{\left[\beta(n)-\rho(n)-c_{\Gamma}\right]^{1-\alpha}}
$$

onde $K=\sup _{\eta \geq 0}\left(\int_{0}^{\eta} u^{-\alpha} e^{\left[\rho(n)+c_{\Gamma}\right](u-\eta)} d u\right)$. Escolha $n_{0}>0$ tal que $\gamma(n) \leq \frac{1}{2}$ para todo $n \geq n_{0}$. Portanto

$$
e^{\beta(n)\left(t-t_{0}\right)}\|\xi(t)\|_{X_{n}^{\alpha}} \leq e^{\beta(n)\left(t-t_{0}\right)} w(t) \leq M_{a}\left\|\xi\left(t_{0}\right)\right\|_{X_{n}^{\alpha}}+\gamma(n) e^{\beta(n)\left(t-t_{0}\right)} w(t)
$$


e

$$
\|\xi(t)\|_{X_{n}^{\alpha}} \leq 2 M_{a}\left\|\xi\left(t_{0}\right)\right\|_{X_{n}^{\alpha}} e^{-\beta(n)\left(t-t_{0}\right)} .
$$

A suavidade de $\sigma_{n}^{*}$ é provada da mesma forma que em [He2] e a estimativa para as derivadas segue da estimativa para suas constantes de Lipschitz. Isto conclui a demonstração.

\subsection{Semi-continuidade superior}

Primeiramente relembraremos a definição de semi-continuidade superior.

Definição 1.11. Seja $\Lambda$ um espaço métrico, $X$ um espaço métrico completo e $J_{\lambda}$ para $\lambda \epsilon$ $\Lambda$ uma familia de subconjuntos de $X$ então dizemos que $J_{\lambda}$ é semi-contínuo superiormente em $\lambda_{0} \in \Lambda$ se $\delta\left(J_{\lambda}, J_{\lambda_{0}}\right) \rightarrow 0$ quando $\lambda \rightarrow \lambda_{0}$, onde $\delta$ é a distância definida em (1.1)

Existem teoremas que nos dão condições suficientes para que a semi-continuidade superior dos atratores ocorra. Estes teoremas podem ser encontrados em [HR2] e [Ha2].

Em nosso estudo não fazemos uso destes teoremas.

Utilizamos uma condição suficiente através de convergência de soluções.

Seja

$$
\frac{d w}{d t}=B_{\lambda} w+f_{\lambda}(w)
$$

uma família de equações, $\operatorname{com} \lambda \in \Lambda$. A família de problemas gera uma família de semigrupos $T_{\lambda}(t): X \rightarrow X$ os quais possuem atrator global $\mathcal{A}_{\lambda}$. Nesta condições temos

Lema 1.2. Seja $\lambda_{k}$ uma sequência de números positivos tal que $\lim _{k \rightarrow \infty} \lambda_{k}=\bar{\lambda}$ e seja $w_{k}(t)$ uma sequência de soluções de (1.48) com $\lambda=\lambda_{k}$ e tal que $w_{k}(t) \in \mathcal{A}_{\lambda_{k}}$ para todo $t \geq 0$. Se existe uma subsequência de $\lambda_{k}$, tal que $w_{k_{j}}(0)$ converge para $\bar{w} \in \mathcal{A}_{\bar{\lambda}}$ em $X$ então a familia de atratores $\mathcal{A}_{\lambda}$ é semi-contínua superiormente em $\lambda=\bar{\lambda}, \mathcal{A}_{\bar{\lambda}}$.

Demonstraçāo: De fato, suponha que a semi-continuidade superior dos atratores não se verifique. Então existe uma constante $\epsilon_{0}$, uma sequência de números positivos $\lambda_{k}$ tal que $\lim _{k \rightarrow \infty} \lambda_{k}=\bar{\lambda}$ e uma correspondente sequência $w_{0 k} \in \mathcal{A}_{\eta_{k}}$ tal que

$$
\delta_{X}\left(w_{0 k}, \mathcal{A}_{\bar{\lambda}}\right) \geq \epsilon_{0}>0, \quad \forall k
$$


Considere $w_{k}(t)$ as soluçōes de (1.48) com $\lambda=\lambda_{k}$ e $w_{k}(0)=w_{0 k}$. Como $w_{k}(t) \in \mathcal{A}_{\lambda_{k}}$ então isto contradiz a hipótese.

Portanto, pelo Lema 1.2, a abordagem utilizada para obter a semi-continuidade superior dos atratores é obter convergência de soluções sobre os atratores. Para isso, primeiramente mostramos estimativas de limitação uniforme dos atratores em um espaço $X$, depois mostramos estimativas de limitação uniforme para os atratores em um espaço $Y$, onde o espaço $Y$ está imerso compactamente no espaço $X$. Com mais uma estimativa uniforme sobre a limitação da derivada em relação ao tempo, podemos fazer uso do Teorema de Arzelà-Ascoli e mostrarmos a convergência de soluções sobre os atratores. A função obtida através desse limite está definida em todo $\mathbb{R}$ e é limitada. Mostramos que esta função é solução do problema limite e portanto está contida no atrator do problema limite. Isto assegura a semi-continuidade superior.

\subsection{Teoremas de semi-continuidade inferior}

Antes de enunciarmos os teoremas de semi-continuidade inferior ramos relembrar a definição

Definição 1.12. Seja $\Lambda$ um espaço métrico, $X$ um espaço métrico completo e $J_{\lambda}$ para $\lambda \in$ $\Lambda$ uma familia de subconjuntos de $X$ então dizemos que $J_{\lambda}$ é semi-contínuo inferiormente em $\lambda_{0} \in \Lambda$ se $\delta\left(J_{\lambda_{0}}, J_{\lambda}\right) \rightarrow 0$ quando $\lambda \rightarrow \lambda_{0}$, onde $\delta$ é a distância definida em (1.1).

Dizemos também que $J_{\lambda}$ é contínua em $\lambda_{0} \in \Lambda$ se $J_{\lambda}$ é semi-contínua superior $e$ inferiormente.

O teorema a seguir é devido a J. Hale e G. Raugel e apareceu pela primeira vez em [HR2]. A demonstração deste teorema segue os argumentos de Hale [Ha2].

Seja $X$ um espaço de Banach e, para $0 \leq \lambda \leq \Lambda$. seja $T_{\lambda}(t) . t \geq 0$ uma família de semigrupos sobre $X$.

Teorema 1.10. Suponhamos que sejam válidas as seguintes hipótese sobre o semigrupo $T_{0}(t)$ para $t \geq 0$

$(H .1)_{0} T_{0}(t), t \geq 0$, é um $C^{1}$ sistema gradiente $e$ assintoticamente suave e órbitas de conjuntos limitados é limitada; 
$(H .2)_{0} O$ conjunto dos pontos de equilibrio, $E_{0}$, de $T_{0}(t)$ é limitado;

$(H .3)_{0}$ Cada equilibrio, $\phi_{j} \in E_{0}$ é hiperbólico

As hipóteses $(H .1)_{0}$ e $(H .2)_{0}$ nos garantem que existe um atrator global $\mathcal{A}_{0}$ e, por $(H .3)_{0}$ temos que $E_{0}=\left\{\phi_{1}, \phi_{2}, \ldots, \phi_{N}\right\} e$

$$
\mathcal{A}_{0}=\bigcup_{j=1}^{N} W^{u}\left(\phi_{j}\right),
$$

onde $W^{u}\left(\phi_{j}\right)$ é a variedade instável de $\phi_{j}$.

Suponhamos que sejam válidas as seguintes hipóteses relativas a continuidade de $T_{\lambda}(t)$ $\operatorname{com} \lambda \rightarrow 0$

$(H .4)_{\lambda} T_{\lambda}(t), t \geq 0$, é um $C^{1}$ semigrupo, é assintoticamente suave e existe uma vizinhança $U_{0}$ de $\mathcal{A}_{0}$ independente de $\lambda$ tal que $T_{\lambda}(t)$ tem um atrator local $\mathcal{A}_{\lambda}$ que atrai $U_{0} ;$

$(H .5)_{\lambda}$ Seja $E_{\lambda}$ o conjunto dos pontos de equilibrio de $T_{\lambda}(t)$. Existe uma vizinhança aberta $W_{0}$ de $E_{0}$ tal que $W_{0} \bigcap E_{\lambda}=\left\{\phi_{1, \lambda}, \phi_{2, \lambda}, \cdots, \phi_{3, \lambda}\right\}, \phi_{j \lambda}$ é hiperbólico e $\phi_{j, \lambda} \rightarrow \phi_{j}$ quando $\lambda \rightarrow 0$

Consideremos as variedades instáveis locais dos pontos de equilibrio, $W_{l o c, \lambda}^{u}\left(\phi_{j, \lambda}\right)$. E suponhamos que $\exists r>0$ tal que $W^{u}\left(\phi_{j, \lambda}\right) \cap B_{3 r / 2}\left(\phi_{j, \lambda}\right) \subset K_{\text {loc, }}^{u}\left(\varphi_{j, \lambda}\right)$. Suponhamos também que

$(H .6)_{\lambda} \delta_{X}\left(W_{l o c, 0}^{u}\left(\phi_{j, 0}\right), W_{l o c, \lambda}^{u}\left(\phi_{j, \lambda}\right)\right) \rightarrow 0$, quando $\lambda \rightarrow 0$

$(H .7)_{\lambda}$ Dados $\eta>0, \tau>0$ e $t_{0}^{*}>0$, existem um número real $\delta^{*}=\delta\left(\eta, \tau, t_{0}^{*}\right)>0$ e um $\lambda_{0}>0$ tal que

$$
\left\|T_{\lambda}(t) y_{\lambda}-T_{0}(t) x\right\|_{X} \leq \eta
$$

para $t_{0}^{*} \leq t \leq \tau, x \in \mathcal{A}, y_{\lambda} \in \mathcal{A}_{\lambda},\left\|x-y_{\lambda}\right\|_{X} \leq \delta^{*} e \lambda \leq \lambda_{0}$.

então temos $\bar{\delta}_{\lambda}\left(\mathcal{A}, \mathcal{A}_{\lambda}\right) \rightarrow 0$ quando $\lambda \rightarrow 0$

Demonstração: Consideramos a decomposiçāo de Norse do atrator $\mathcal{A}_{0}$ para $T_{0}(t)$.

Seja $E_{0}=\left\{o_{1}, \phi_{2}, \cdots, \phi_{N}\right\}$ o conjunto dos pontos de equilíbrio de $T_{0}(t)$, e sejam $\imath^{1}>$ $r^{2}>\cdots>q^{M}$ os pontos distintos do conjunto $\left\{I^{*}\left(\dot{\phi}_{1}\right), V\left(\dot{\phi}_{2}\right) \cdots, \Gamma^{*}\left(\phi_{N}\right)\right\}$. Definimos

$$
E_{0}^{j}=\left\{x \in E_{0}: \Upsilon^{\mathcal{V}}(x)=v^{j}\right\}
$$




$$
\begin{gathered}
A_{0}^{k}=\bigcup\left\{W^{u}(x) ; x \in \bigcup_{j=k}^{M} E_{0}^{j}\right\} \\
U_{0}^{k}=\left\{x \in X, V(x)<v^{k}\right\} .
\end{gathered}
$$

Se $\phi_{j}$ é um equilíbrio hiperbólico de $T_{0}(t)$, então existe um $r>0$ tal que $N_{r}\left(\phi_{j}\right) \cap W^{u}\left(\phi_{j}\right)$ $\subset W_{l o c}^{u}\left(\phi_{j}\right)$ e se

$$
\Gamma_{j}^{r}=W_{l o c}^{u}\left(\phi_{j}\right) \bigcap \partial N_{r}\left(\phi_{j}\right)
$$

então

$$
W^{u}\left(\phi_{j}\right)=W_{l o c}^{u}\left(\phi_{j}\right) \bigcup\left(\bigcup_{t \geq 0} T_{0}(t) \Gamma_{j}^{r}\right)
$$

Escolhemos $r$ tal que $N_{2 r}\left(\phi_{j}\right) \bigcap N_{2 r}\left(\phi_{k}\right)=\emptyset$ para $j \neq k$. Desde que $W_{l o c}^{u}\left(\phi_{j}\right)$ é de dimensão finita então os conjuntos $\Gamma_{j}^{r}, j=1, \cdots N$ são compactos. Da definição de sistema gradiente, para qualquer inteiro $k, 1 \leq k \leq M-1$ existe um $t_{k}>0$ tal que, para qualquer $\phi_{j k} \in E_{0}^{k}$,

$$
V\left(T_{0}(t) \Gamma_{j k}^{r}\right) \leq v^{k+1}+\frac{d}{2}
$$

para $t \geq t_{k}$, onde $d=\min \left\{v^{i-1}-v^{i}, i=2, \cdots, M\right\}$.

Seja

$$
t_{0}=\max \left\{t_{k}, k=1, \cdots M\right\}
$$

Dado $\epsilon>0$, é claro que se para qualquer $x \in \mathcal{A}_{0}$, temos

$$
\inf _{y \in \mathcal{A}_{\lambda}}\left\|T_{0}(t) x-y\right\|_{X} \leq \epsilon, \quad t \geq 0
$$

então, em particular, para $t=0 . \mathrm{E}$ assim o teorema está provado.

Logo, mostraremos que para qualquer $x \in \mathcal{A}_{0}$, temos

$$
\inf _{y \in \mathcal{A}_{\lambda}}\left\|T_{0}(t) x-y\right\|_{X} \leq \epsilon, \quad t \geq 0
$$

Para $x \in \mathcal{A}_{0}$ temos duas possibilidades.

i) Se $x \in E_{0}$ então $x=\phi_{j}$ para algum $j$. Por $(H 5)_{\lambda}$, temos que $\left\|\phi_{j, \lambda}-\phi_{j}\right\| \leq \epsilon$ para $\epsilon \leq \epsilon_{0}$ e $\phi_{j, \lambda} \in E_{\lambda} \subset \mathcal{A}_{\lambda}$.

ii) Se $x \notin E_{0}$ então $x \in W^{u}\left(\phi_{j}\right)$ para algum $\phi_{j} \in E_{0}$. Desde que $W^{u}\left(\phi_{j}\right)=W_{l o c}^{u}\left(\phi_{j}\right) \bigcup$ $\left(\bigcup_{t \geq 0} T_{0}(t) \Gamma_{j}^{r}\right)$ então existe $s_{j}$ tal que $x=T_{0}\left(s_{j}\right) \phi_{j}^{*}$ onde $\phi_{j}^{*} \neq \phi_{j}$ e $\phi_{j}^{*} \in W_{l o c}^{u}\left(\phi_{j}\right) \cap N_{r}\left(\phi_{j}\right)$. 


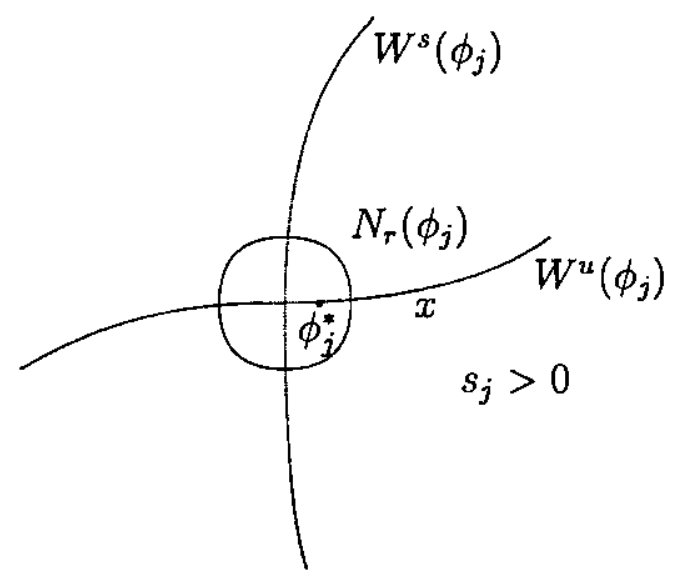

Assim, se $s_{j}$ dado acima é tal que $s_{j} \geq 0$, ou seja, $x \notin W_{l o c}^{u}\left(\phi_{j}\right) \bigcap N_{r}\left(\phi_{j}\right)$ então

$$
\inf _{y \in \mathcal{A}_{\lambda}}\left\|T_{0}(t) \phi_{j}^{*}-y\right\|_{X} \leq \epsilon, \quad t \geq 0
$$

implica

$$
\inf _{y \in \mathcal{A}_{\lambda}}\left\|T_{0}(t) x-y\right\|_{X} \leq \epsilon, \quad t \geq 0
$$

Se $s_{j} \leq 0$

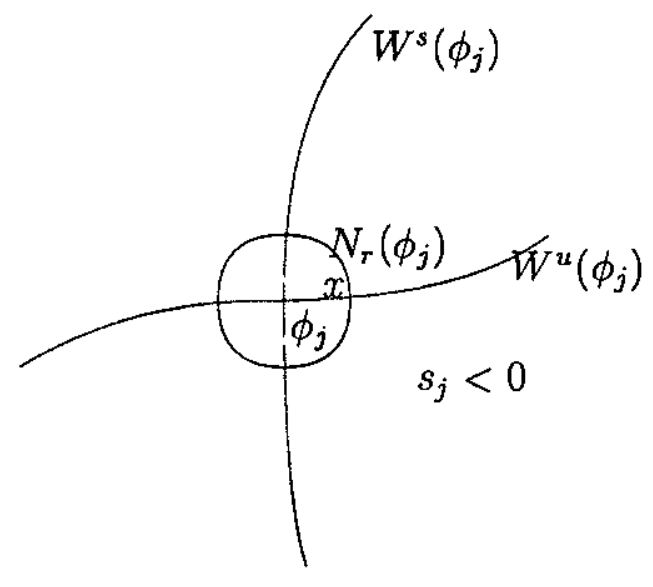

então $x \in K_{l o c}^{u}\left(\phi_{j}\right) \bigcap N_{r}\left(\phi_{j}\right)$.

Logo, por $(H 6)_{\lambda}$, existe um número real $\lambda_{1} \leq \lambda_{0}$ tal que, para $0 \leq \lambda \leq \lambda_{1}$ nós temos para $1 \leq j \leq N$

$$
\delta_{X}\left(W_{l o c}^{u}\left(\phi_{j}\right), W_{l o c}^{u}\left(\phi_{j, \lambda}\right)\right) \leq \epsilon
$$


e assim,

$$
\inf _{y \in \mathcal{A}_{\lambda}}\left\|T_{0}(t) x-y\right\|_{X} \leq \epsilon, \quad 0 \leq t \leq-s_{j}
$$

e assim, basta mostrar

$$
\inf _{y \in \mathcal{A}_{\lambda}}\left\|T_{0}(t) \phi_{j}^{*}-y\right\|_{X} \leq \epsilon, \quad t \geq 0
$$

Portanto, em qualquer dos casos é suficiente provar que, para $1 \leq j \leq N$ e para qualquer $\phi_{j}^{*} \in \Gamma_{j}^{r}$ temos:

$$
\inf _{y \in \mathcal{A}_{\lambda}}\left\|T_{0}(t) \phi_{j}^{*}-y\right\|_{X} \leq \epsilon, \quad t \geq 0
$$

Sem perda de generalidade, é suficiente fazer a demonstração de (1.58) somente para o caso em que $\phi_{j}=\phi_{j 1} \in E^{1}$ e $\phi_{j 1}^{*} \in \Gamma_{j 1}^{r}$. A demonstração é feita por indução e consiste de no máximo $M$ passos pois vamos identificar em qual dos $M$ níveis de energia o conjunto $\omega$-limite de $\phi_{j 1}^{*}, \omega\left(\phi_{j 1}^{*}\right)$, estará.

Primeiramente vamos fazer algumas consideraçōes.

Desde que $\mathcal{A}_{0}$ é compacto e $V$ é continua então $V$ é uniformemente continua sobre $\mathcal{A}_{0}$, ou seja, existe uma constante $\epsilon_{d}$ tal que, para quaisquer $x_{1}, x_{2} \in \mathcal{A}_{0}$ com $\left\|x_{1}-x_{2}\right\| \leq \epsilon_{d}$ temos

$$
\left|V\left(x_{1}\right)-V\left(x_{2}\right)\right| \leq \frac{d}{4}
$$

Defina $B_{k}=\left\{x \in \mathcal{A}_{0} ; V(x) \leq v^{k}-\frac{d}{4}\right\}$ e fixe uma constante $t_{0}^{*}>0$. Construiremos duas sequências de números reais:

$$
\begin{aligned}
& \epsilon_{M-1}>\epsilon_{M-2}>\cdots>\epsilon_{0} \\
& \lambda_{M-1}>\lambda_{M-2}>\cdots>\lambda_{0}
\end{aligned}
$$

da seguinte forma. Seja $\epsilon_{M-1}=\min \left\{\frac{\epsilon}{2}, \frac{\epsilon_{d}}{2}, \frac{\tau}{2}\right\}$ e escolha $\lambda_{M-1}<\lambda_{0}^{*}$ tal que $\left\|\phi_{j}-\phi_{j, \lambda}\right\|<$ $\epsilon_{M-1}$ para $1 \leq j \leq N$ e $\lambda \leq \lambda_{M-1}$.

Suponha $k \geq 1$ e assuma que $\epsilon_{M-1}>\epsilon_{M-2}>\cdots>\epsilon_{k}$ e $\lambda_{M-1}>\lambda_{M-2}>\cdots>\lambda_{k}$ tenham sido escolhidos. Como $\mathcal{A}_{0}^{k+1}$ é um atrator para $T_{0}(t)$ restrito a $U_{0}^{k}$ e $B_{k} \subset U_{0}^{k}$, então, existe um $\tau_{k}$ tal que para $t \geq \tau_{k}$ temos que

$$
T_{0}(t) B_{k} \subset N_{\epsilon_{k}}\left(\mathcal{A}_{0}^{k+1}\right)
$$


Da representação de $\mathcal{A}_{0}^{k+1}$, nós deduzimos que, para qualquer $\phi \in B_{k}$, existe um $\psi \in$ $\bigcup_{j=k+1}^{M} E^{j}$ tal que

$$
\delta_{X}\left(T_{0}\left(\tau_{k}\right) \phi, W^{u}(\psi)\right) \leq \epsilon_{k}
$$

Seja $t_{0}$ o número definido pela fórmula (1.50). Desde que $T_{0}(t)$ é um $C^{0}$-semigrupo, existe um $\epsilon_{k-1}^{*}>0$ tal que

$$
\left\|T_{0}(t) x-T_{0}(t) x^{\prime}\right\|_{X}<\epsilon_{k}, \text { para } 0 \leq t \leq\left(t_{0} \tau_{k}\right)+2 t_{0}^{*}
$$

para $x, x^{\prime} \in \mathcal{A}_{0}$ e $\left\|x-x^{\prime}\right\|_{X} \leq 2 \epsilon_{k-1}^{*}$.

$\operatorname{Por}(H 7)_{\lambda}$, existem números reais positivos $\epsilon_{k-1} \leq \min \left\{\epsilon_{k-1}^{*}, \frac{\epsilon_{k}}{2}\right\}$ e $\lambda_{k-1}^{*}<\lambda_{k}$ tal que, para $0<\lambda \leq \lambda_{k-1}^{*}$, temos:

$$
\left\|T_{\lambda}(t) y_{\lambda}-T_{0}(t) x\right\|_{X} \leq \epsilon_{k}, \quad \text { para } t_{0}^{*} \leq t \leq\left(t_{0}+\tau_{k}\right)+2 t_{0}^{*}
$$

desde que $x \in \mathcal{A}_{0}, y_{\lambda} \in \mathcal{A}_{\lambda}$ e $\left\|y_{\lambda}-x\right\| \leq 2 \epsilon_{k-1}$.

Por $(H 6)_{\lambda}$, nós podemos escolher $0<\lambda_{k-1}<\lambda_{k-1}^{*}$ tal que, para $0<\lambda<\lambda_{k-1}$,

$$
\delta_{X}\left(W_{l o c}^{u}\left(\phi_{j}\right), W_{l o c}^{u}\left(\phi_{j, \lambda}\right) \leq \epsilon_{k-1}, \quad \text { para } 1 \leq j \leq N\right.
$$

Isto completa a construção das sequências $\epsilon_{k}$ e $\lambda_{k}$.

Nós agora provaremos por indução que, para $0<\lambda \leq \lambda_{0}$ e para qualquer $\phi_{j 1} \in E^{1} \mathrm{e}$ $\phi_{j h 1}^{*} \in \Gamma_{j 1}^{r}$ temos 1.58 , ou seja,

$$
\inf _{y \in \mathcal{A}_{\lambda}}\left\|T_{0}(t) \phi_{j}^{*}-y\right\|_{X} \leq \epsilon, \quad t \geq 0
$$

$1^{\circ}$ passo:

Por (1.49), temos $T\left(t_{1}\right) \Gamma_{j 1}^{r} \subset B_{1}$ e por (1.61) temos $T_{0}\left(t_{1}+t\right) \Gamma_{j 1}^{r} \subset N_{\epsilon_{1}}\left(\mathcal{A}_{0}^{2}\right)$ para $t \geq \tau_{1}$. Seja $\bar{\phi}_{j 1}^{*} \in W_{l o c}^{u}\left(\phi_{j 1}\right)$ tal que $T_{0}\left(t_{0}\right) \bar{\phi}_{j 1}^{*}=\phi_{j 1}^{*}$. Então, por (1.64) temos que existe um $\tilde{\phi}_{j 1, \lambda}^{*} \in W_{l o c, \lambda}^{u}\left(\phi_{j 1, \lambda}\right)$ tal que

$$
\left\|\tilde{\phi}_{j 1, \lambda}^{*}-\tilde{\phi}_{j 1}^{*}\right\| \leq \lambda_{0}
$$

e por (1.63), para $t_{0}^{*}=t_{0}$, temos

$$
\left\|T_{0}\left(t_{0}+t\right) \tilde{\phi}_{j 1}^{*}-T_{\lambda}\left(t_{0}+t\right) \tilde{\phi}_{j 1, \lambda}^{*}\right\| \leq \epsilon_{1}, \quad \text { para } 0 \leq t \leq\left(t_{1}+\tau_{1}\right)+t_{0}^{*} .
$$

Como, $T_{0}\left(t_{0}\right) \tilde{\phi}_{j 1}^{*}=\phi_{j 1}^{*}$ e $T_{\lambda}\left(t_{0}+t\right) \tilde{\phi}_{j 1, \lambda}^{*} \in \mathcal{A}_{\lambda}$ para todo $0 \leq t \leq\left(t_{1}+\tau_{1}\right)+t_{0}^{*}$ então, escrevendo $s_{1}=t_{1}+\tau_{1}$, temos:

$$
\inf _{y \in \mathcal{A}_{\lambda}}\left\|T_{0}(t) \phi_{j 1}^{*}-y\right\|_{X} \leq \epsilon_{1}, \quad 0 \leq t \leq s_{1}+t_{0}
$$


e $T_{0}\left(s_{1}+s\right) \phi_{j 1}^{*} \in N_{\epsilon_{1}}\left(\mathcal{A}^{2}\right)$ para $s \geq 0$

$k^{o}$ passo:

Vamos assumir que existe um $s_{k-1}>0$ tal que

$$
T_{0}\left(s_{1}+s\right) \phi_{j 1}^{*} \in N_{\epsilon_{1}}\left(\mathcal{A}^{2}\right), \text { para } s \geq 0
$$

e

$$
\inf _{y \in \mathcal{A}_{\lambda}}\left\|T_{0}(t) \phi_{j 1}^{*}-y\right\|_{X} \leq \epsilon_{k-1}, \text { para } 0 \leq t \leq s_{k-1}+t_{0}
$$

Por (1.66) para $t=s_{k-1}$ temos que existe um $y_{\lambda} \in \mathcal{A}_{\lambda}$ tal que $\left\|T_{0}\left(s_{k-1}\right) \phi_{j 1}^{*}-y_{\lambda}\right\|_{X} \leq 2 \epsilon_{k-1}$ e por (1.63), temos que

$$
\left\|T_{0}\left(t+s_{k-1}\right) \phi_{j 1}^{*}-T_{\lambda}(t) y_{\lambda}\right\|_{X} \leq \epsilon_{k}, \quad \text { para } t_{0} \leq t \leq\left(t_{0}+\tau_{k}\right)+2 t_{0} .
$$

Logo,

$$
\inf _{y \in \mathcal{A}_{\lambda}}\left\|T_{0}\left(t+s_{k-1}\right) \phi_{j 1}^{*}-y\right\|_{X} \leq \epsilon_{k}, \quad \text { para } \quad t_{0} \leq t \leq\left(t_{0}+\tau_{k}\right)+2 t_{0} .
$$

Portanto,

$$
\inf _{y \in \mathcal{A}_{\lambda}}\left\|T_{0}(t) \phi_{j 1}^{*}-y\right\|_{X} \leq \epsilon_{k}, \quad \text { para } \quad t_{0}+s_{k-1} \leq t \leq s_{k-1}+\left(t_{0}+\tau_{k}\right)+2 t_{0}
$$

A assim, por (1.66) e por (1.67) temos

$$
\inf _{y \in \mathcal{A}_{\lambda}}\left\|T_{0}(t) \phi_{j 1}^{*}-y\right\|_{X} \leq \epsilon_{k}, \quad \text { para } 0 \leq t \leq s_{k-1}+\left(t_{0}+\tau_{k}\right)+2 t_{0}
$$

De (1.65) temos que para cada $s \geq 0$ existe um $\phi_{j k}^{s} \in \bigcup_{j=k}^{M} E^{j}$ e $\tilde{\phi}_{j k}^{s} \in W^{u}\left(\phi_{j k}^{s}\right)$ tal que

$$
\left\|T_{0}\left(s_{k-1}+s\right) \phi_{j 1}^{*}-\tilde{\phi}_{j k}^{s}\right\| \leq \epsilon_{k-1}
$$

Vamos por primeiro $s=0$.

Se $\tilde{\phi}_{j k}^{0} \in W_{l o c}^{u}\left(\phi_{j k}^{0}\right) \cap\left\{y \in X ;\left\|y-\phi_{j k}^{0}\right\| \leq r\right\}$ então temos

$$
\left\|T_{0}\left(s_{k-1}\right) \phi_{j 1}^{*}-\phi_{j k}^{0}\right\| \leq \epsilon_{k-1}+r \leq \frac{3 r}{2}
$$

e existe um número real $\sigma_{0}>0$ tal que $T_{0}\left(s_{k-1}+s\right) \phi_{j 1}^{*} \in N_{2 r}\left(\phi_{j k}^{0}\right)$ para $0 \leq s \leq$ $\sigma_{0}$. Além disso, se para um $s$ em $\left(0, \sigma_{0}\right]$, o elemento $\tilde{\phi}_{j k}^{s}$ dado em $(1.69)$ : pertence a $W_{l o c}^{u}\left(\phi_{j k}^{s}\right) \bigcap N_{r}\left(\phi_{j k}^{s}\right)$ então $\phi_{j k}^{s}=\phi_{j k} 0$ desde que $N_{2 r}\left(\phi_{j}\right) \bigcap N_{2 r}\left(\phi_{j}\right)=\emptyset$ para $j \neq k$. Com esta propriedade temos somente duas possibilidades: 
ou a) $\tilde{\phi}_{j k}^{s} \in W_{l o c}^{u}\left(\phi_{j k}^{0}\right) \bigcap N_{r}\left(\phi_{j k}^{0}\right)$ para todo $s \geq 0$, ou seja, se o $\omega$-limite de $\phi_{j 1}^{*}, \omega\left(\phi_{j 1}^{*}=\phi_{j k}^{0}\right.$. Então, por (1.64), temos que para $t \geq s_{k-1}$

$$
\inf _{y \in \mathcal{A}_{\lambda}}\left\{T_{0}(t) \phi_{j 1}^{*}-y\right\} \leq 2 \epsilon_{k-1}
$$

e a hipótese de indução (1.66) nos dá para $t \geq 0$ que

$$
\inf _{y \in \mathcal{A}_{\lambda}}\left\{T_{0}(t) \phi_{j 1}^{*}-y\right\} \leq 2 \epsilon_{k-1} .
$$

ou b) existe um primeiro tempo $\sigma \geq 0$ tal que o elemento $\tilde{\phi}_{j k}^{\sigma}$ dado em (1.69) não pertence a $W_{l o c}^{u}\left(\phi_{j k}^{0}\right) \bigcap N_{r}\left(\phi_{j k}^{0}\right)$ e se, $\sigma>0, \tilde{\phi}_{j k}^{s} \in W_{l o c}^{u}\left(\phi_{j k}^{0}\right) \bigcap N_{r}\left(\phi_{j k}^{0}\right)$ para $0 \leq s<\sigma$ e $\tilde{\phi}_{j k}^{\sigma} \in$ $W^{u}\left(\phi_{j k}^{0}\right) \cap \Gamma^{r}\left(\phi_{j k}^{0}\right)$.

Assim, para $s \geq t_{k}, V\left(T_{0}(s) \tilde{\phi}_{j k}^{\sigma}\right) \leq v^{k+1}+\frac{d}{2}$. De (1.69) temos

$$
\left\|T_{0}\left(s_{k-1}+s\right) \phi_{j 1}^{*}-\tilde{\phi}_{j k}^{s}\right\| \leq \epsilon_{k-1}
$$

para $s \geq 0$ e assim, para $s=\sigma$ e por (1.62) temos

$$
\left\|T_{0}\left(s_{k-1}+\sigma+t\right) \phi_{j 1}^{*}-T_{0}(t) \tilde{\phi}_{j k}^{s}\right\| \leq \epsilon_{k}, \quad \text { para } 0 \leq t \leq t_{k}
$$

pois $t_{k} \leq\left(t_{0}+\tau_{k}\right)+2 t_{0}$.

Como $\epsilon_{k} \leq \epsilon_{d}$ então para $t=t_{k}$ temos por (1.59) temos

$$
\left|V\left(\mid T_{0}\left(s_{k-1}+\sigma+t_{k}\right) \phi_{j 1}^{*}\right)-V\left(T_{0}(t) \tilde{\phi}_{j k}^{s}\right)\right| \leq \frac{d}{4}
$$

e assim, $V\left(T_{0}\left(s_{k-1}+\sigma+t_{k}\right) \phi_{j 1}^{*}\right) \leq V\left(T_{0}(t) \tilde{\phi}_{j k}^{s}\right)+\frac{d}{4} \leq v^{k}-\frac{d}{4}$. Logo, $V\left(T_{0}\left(s_{k-1}+\sigma+t_{k}\right) \phi_{j 1}^{*}\right) \in$ $B_{k}$ e desde que $V$ é não crescente ao longo de órbitas então $V\left(T_{0}\left(s_{k-1}+\sigma+t\right) \phi_{j 1}^{*}\right) \in B_{k}$ para $t \geq t_{k}$. E portanto, por (1.61)

$$
\left.T_{0}\left(s_{k}+s\right) \phi_{j 1}^{*}\right) \in N_{\epsilon_{k}}\left(\mathcal{A}_{0}^{k+1}\right), \text { para } s \geq 0
$$

onde $s_{k}=s_{k-1}+\sigma+t_{k}+\tau_{k}$.

$E$ assim, a hipótese de indução (1.65) está provada.

Resta provar que (1.66) com $k-1$ trocado por $k$, ou seja,

$$
\inf _{y \in \mathcal{A}_{\lambda}}\left\|T_{0}(t) \phi_{j 1}^{*}-y\right\|_{X} \leq \epsilon_{k}, \text { para } 0 \leq t \leq s_{k}+t_{0}
$$

Primeiramente, observamos que se $\sigma \leq t_{0}$ então (1.73) é o mesmo que (1.68). Assim, supomos que $\sigma \geq t_{0}$. Por (1.64), para $0 \leq s<\sigma$, existe $\tilde{\phi}_{j k, \lambda}^{s} \in W_{l o c, \lambda}^{u}\left(\phi_{j k, \lambda}^{0}\right)$ tal que

$$
\left\|\tilde{\phi}_{j k, \lambda}^{s}-\tilde{\phi}_{j k}^{s}\right\| \leq \epsilon_{k-1}
$$


Por (1.69) temos que $\exists \tilde{\phi}_{j k}^{s} \in W_{l o c}^{u}\left(\phi_{j k}^{s}\right)$ tal que $\left\|T_{0}\left(s_{k-1}+s\right) \phi_{j 1}^{*}-\tilde{\phi} s_{j k}\right\| \leq \epsilon_{k-1}$. E por (1.74), temos $\left\|\tilde{\phi} s_{j k, \lambda}-\tilde{\phi} s_{j k}\right\| \leq \epsilon_{k-1} \log 0$

$$
\left\|\tilde{\phi}_{j k, \lambda}^{s}-T_{0}\left(s_{k-1}+s\right) \phi_{j 1}^{*}\right\| \leq 2 \epsilon_{k-1} \leq \epsilon_{k}
$$

Como consequência de (1.63) e (1.75) temos para $s=\sigma-t_{0}>0$ que

$$
\left\|T_{0}\left(s_{k-1}+\sigma-t_{0}+t\right) \phi_{j 1}^{*}-T_{\lambda}(t) \tilde{\phi}_{j k, \lambda}^{\sigma-t_{0}}\right\| \leq \epsilon_{k}, \quad \text { para } t_{0} \leq t \leq\left(t_{0}+\tau_{k}\right)+2 t_{0}
$$

E assim, para $0 \leq t \leq s_{k-1}+t_{0}$ temos pela (1.66), que $\inf _{y \in \mathcal{A}_{\lambda}}\left\|T_{0}(t) \phi_{j 1}^{*}-y\right\|_{X} \leq \epsilon_{k}$. Para $s_{k-1}+t_{0} \leq t \leq s_{k-1}+\sigma$ temos, por (1.75) que $\inf _{y \in \mathcal{A}_{\lambda}}\left\|T_{0}(t) \phi_{j 1}^{*}-y\right\|_{X} \leq \epsilon_{k}$ e finalmente, para $s_{k-1}+t_{0} \leq t \leq s_{k}+t_{0}$, por (1.76) temos $\inf _{y \in \mathcal{A}_{\lambda}}\left\|T_{0}(t) \phi_{j 1}^{*}-y\right\|_{X} \leq \epsilon_{k}$. Logo, a hipótese de indução (1.73) está provada.

$M^{\circ}$ Passo: Finalmente, argumentando como anteriormente $M-2$ vezes no máximo, mostra-se que:

ou existe um $k_{0} \leq M-1$ tal que $\omega\left(\phi_{j 1}^{*}\right) \subset E^{k_{0}}$ e então (1.71) é assegurado para $k=k_{0}$; ou, pela hipótese de indução, existe $s_{M-1}>0$ tal que $T_{0}\left(s_{M-1}+s\right) \phi_{j 1}^{*} \in N_{\epsilon_{M-1}}\left(E^{M}\right)$ para $s \geq 0$ e (1.71) é assegurado. Mas $W_{l o c}^{u}\left(\phi_{j M}\right)=\phi_{j M}$ onde $\phi_{j M} \in E^{M}$. Assim, por (1.64), temos

$$
\inf _{y_{\lambda} \in \mathcal{A}_{\lambda}}\left\|T_{0}\left(s_{M-1}+s\right) \phi_{j 1}^{*}-y_{\lambda}\right\| \leq 2 \epsilon_{M-1} \leq \epsilon_{M} \leq \epsilon
$$

para $s \geq 0$.

OBSERVAÇŌES: Se no teorema tivermos dois parâmetros $\lambda$ e $n$ e desejarmos analisar a semi-continuidade inferior relativamente a um parâmetro, $\lambda$, mas uniforme no segundo parâmetro, $n$, então a uniformidade das hipóteses do teorema relativamente ao segundo parâmetro não é suficiente para garantir o resultado pois, como pode ser observado na demonstração, a construção das sequências $\epsilon_{k}$ e $\lambda_{k}$ dependem também do $t_{0}$ definido em (1.50) e este está vinculado ao decrescimento do funcional $T^{\circ}$ : o qual não está envolvido nas hipóteses quando existe apenas um parâmetro.

Logo, para obtermos a semi-continuidade inferior uniforme em outro parâmetro, com hipóteses já uniformes, é também necessário que o $t_{0}$ seja escolhido independente deste novo parâmetro. 
O próximo teorema nos dá condições suficientes para que a hipótese $(H 6)_{\lambda}$ seja verificada. Como referência para este teorema temos $[\mathrm{CH}]$.

Fixamos um ponto de equilíbrio $\left(\phi_{j, 0}\right)$ e transladamos todos os equilíbrios de forma que o equilíbrio fixado seja a origem. Continuaremos a denotar o equilíbrio transladado por $\phi_{j, \lambda}$. Para cada $\lambda \geq 0$ e $\phi_{j, \lambda}$ sejam $S_{j \lambda}$ e $U_{j \lambda}$ as variedades estável e instável de $\phi_{j \lambda}$ do semigrupo linear (gerado pela linearização em $\phi_{j, \lambda}$ ), respectivamente. Sejam $P_{j \lambda}$ e $Q_{j \lambda}$ as projeções espectrais sobre $U_{j \lambda}$ e $S_{j \lambda}$ respectivamente. E seja $W_{j \lambda}$ a variedade instável de $\phi_{j \lambda}$ do semigrupo não linear. Consideremos a vizinhança $N_{j \lambda}$ de $\phi_{j \lambda}$ e um homeomorfismo $\sigma_{j \lambda}: U_{j \lambda} \cap N_{j \lambda} \rightarrow N_{j \lambda} \cap W_{j \lambda}$. Como observado em [CH], temos que a distância entre o fluxo e a variedade instável é limitada superiormente pela distância do fluxo a variedade instável na direção da variedade estável, isto é, $\left\|Q_{j \lambda} T_{\lambda}(t) x_{0}-\sigma\left(P_{j \lambda} T_{\lambda}(t) x_{0}\right)\right\|$ :

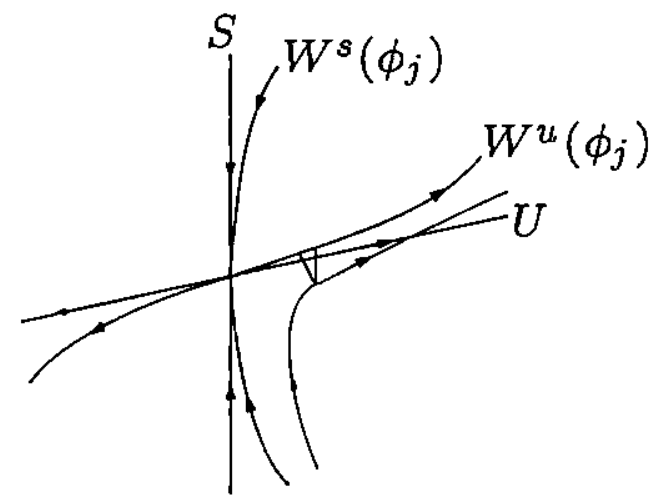

Com estas considerações temos o seguinte teorema:

Teorema 1.11. Suponhamos que $T_{\lambda}(t)$ satisfaz as seguintes hipóteses

(J1) Dado $\eta>0, \tau>0$ e $t_{0}^{*}>0$, existe um $\lambda_{0}>0$ tal que

$$
\left\|T_{\lambda}(t) x-T_{0}(t) x\right\|_{X} \leq \eta
$$

para $t_{0}^{*} \leq t \leq \tau, x \in \mathcal{A}$ e $\lambda \leq \lambda_{0}$

(J2) Cada $\phi_{j, \lambda}$ é hiperbólico e $\phi_{j, \lambda} \rightarrow \phi_{j, 0}$ quando $\lambda \rightarrow 0$, ou seja, dado $\eta>0$, existe um $\lambda_{0}$ tal que $\left\|\phi_{j, \lambda}-\phi_{j, 0}\right\|_{\lambda} \leq \eta$

(J3) Existem números reais positivos $p$ e $q$ tais que $\left\|P_{j \lambda}\right\| \leq p$ e $\left\|Q_{j \lambda}\right\| \leq q$, para $\lambda \leq \lambda_{0}$,

(J4) As constantes de Lipschitz de $\sigma_{j \lambda}, L_{j \lambda}$, são independentes de $\lambda$, 
(J5) Existe $u m \delta_{1}>0$ independente de $\lambda$ tal que $B_{\delta_{1}}\left(\phi_{j \lambda}\right) \subset N_{j \lambda}$ para $\lambda \leq \Lambda$

(J6) Existem um $\delta_{2}$ e um $M$ tais que para $\lambda \leq \Lambda$ temos

$$
\| Q_{j \lambda} T_{\lambda}(t) x_{0}-\sigma_{j \lambda}\left(P_{j \lambda} T_{\lambda}(t) x_{0}\left\|\leq M e^{-\beta t}\right\| Q_{j \lambda} x_{0}-\sigma_{j \lambda}\left(P_{j \lambda} x_{0}\right) \|\right.
$$

onde $\beta>0$ e pode depender de $\lambda$.

sejam $\delta=\min \left\{\delta_{1}, \delta_{2}\right\}$ e $r=\max \{1, p\}$ e definamos $\rho=\frac{\delta}{2 r}$. Então as variedades $V_{j \lambda}=$ $W_{j \lambda} \bigcap B_{\rho}\left(\phi_{j \lambda}\right)$ são semi-contínuas inferiormente em $\lambda=0$.

Demonstração: Desde que $\phi_{j 0}$ está fixado então omitimos o índice $j$. Desde que $W_{0}$ é de dimensão finita e $V_{0}=W_{0} \bigcap B_{\rho}\left(\phi_{0}\right)$ então $V_{0}$ é compacto. Se dado $\eta>0$ e $y \in V_{0}$ existe um $\bar{\lambda}$ tal que $y \in N_{\eta}\left(V_{\lambda}\right)$ para $\lambda \leq \bar{\lambda}$. Então como $V_{0}$ é compacto, escolhemos $\lambda_{0}$ independente de $y \in V_{0}$, ou seja, escolhemos $\lambda_{0}$ tal que $V_{0} \subset N_{\eta}\left(V_{\lambda}\right)$ para $\lambda \leq \lambda_{0}$.

Dado $\eta>0$ defina $\bar{\eta}=\min \left\{\frac{\eta}{K}, \frac{\rho}{2}\right\}$ onde $K=1+M q+2 M p L_{\sigma}+M$. Escolhemos $\tau>0$ tal que $T_{0}(-\tau) V_{0} \subset B_{\bar{\eta}}\left(\phi_{0}\right)$. Pela hipótese (J1) existe $\lambda_{1}$ tal que $\left\|T_{\lambda}(t) x-T_{0}(t) x\right\|_{X} \leq \bar{\eta}$ para $0 \leq t \leq \tau, x \in \mathcal{A}$ e $\lambda \leq \lambda_{1}$. E pela hipótese (J2), existe um $\lambda_{2}$ tal que $\phi_{\lambda} \in B_{\bar{\eta}}\left(\phi_{0}\right)$ para todo $\lambda \leq \lambda_{2}$. Consideramos $\lambda=\min \left\{\lambda_{1}, \lambda_{2}\right\}$. Para cada $y \in V_{0}$, definamos $x=$ $T_{0}(-\tau) y$. Então:

$$
\left.d\left(y, V_{\lambda}\right) \leq d\left(y, T_{\lambda}(\tau) x\right)+d\left(T_{\lambda}(\tau) x\right) . \overline{V_{\lambda}}\right)
$$

Mas,

$$
\left.d\left(T_{\lambda}(\tau) x\right), V_{\lambda}\right) \leq\left\|Q_{\lambda} T_{\lambda}(t) x-\sigma_{\lambda}\left(P_{\lambda} T_{\lambda}(t) x_{0}\right)\right\|
$$

como observamos anteriormente.

Desde que $x \in B_{\bar{\eta}}\left(\phi_{0}\right)$ e $\phi_{\lambda} \in B_{\tilde{\eta}}\left(\phi_{0}\right)$ então $x \in B_{\rho}\left(\phi_{\lambda}\right)$ e portanto $\left\|P_{\lambda} x-\phi_{\lambda}\right\|=$ $\left\|P_{\lambda} x-P_{\lambda} \phi_{\lambda}\right\| \leq p\left\|x-\phi_{\lambda}\right\| \leq p \rho \leq \delta$, ou seja. $P_{\lambda} x \in B_{\delta}\left(\phi_{\lambda}\right)$.

Também, por $(\mathrm{J} 1),\left\|T_{\lambda}(\tau) x-T_{0}(\tau) x\right\| \leq \frac{\delta}{4 r}$ e $T_{0}(\tau) x \in B_{\frac{\delta}{2 \tau}}\left(\phi_{0}\right)$ então $T_{\lambda}(\tau) x \in B_{\frac{3 \delta}{4 r}}\left(\phi_{0}\right)$. Logo $\left\|T_{\lambda}(\tau) x-\phi_{\lambda}\right\| \leq \frac{3 \delta}{4 r}+\bar{\eta} \leq \delta$, ou seja, $T_{\lambda}(\tau) x \in B_{\delta}\left(\phi_{\lambda}\right)$. 
Portanto, por (J1), (J3), (J4), (J6) e (1.78) temos de (1.77) que

$$
\begin{aligned}
d\left(y, V_{\lambda}\right) & \leq\left\|T_{0}(\tau) x-T_{\lambda}(\tau) x\right\|+\| Q_{\lambda} T_{\lambda}(t) x-\sigma_{\lambda}\left(P_{\lambda} T_{\lambda}(t) x_{0} \|\right. \\
& \leq\left\|T_{0}(\tau) x-T_{\lambda}(\tau) x\right\|+M \| Q_{j \lambda} x_{0}-\sigma_{j \lambda}\left(P_{j \lambda} x_{0} \|\right. \\
& \leq \bar{\lambda}+M\left\|Q_{\lambda} x\right\|+M\left\|\sigma_{\lambda}\left(P_{\lambda} x\right)-\sigma_{\lambda}\left(P_{\lambda} \phi_{\lambda}\right)\right\|+M\left\|\sigma_{\lambda}\left(P_{\lambda} \phi_{\lambda}\right)\right\| \\
& \leq \bar{\lambda}+M q\|x\|+M L_{\sigma}\left\|\left(P_{\lambda} x\right)-\left(P_{\lambda} \phi_{\lambda}\right)\right\|+M\left\|\left(P_{\lambda} \phi_{\lambda}\right)\right\| \\
& \leq \bar{\lambda}+M q\|x\|+M p L_{\sigma}\left\|x-\phi_{\lambda}\right\|+M\left\|\phi_{\lambda}\right\|
\end{aligned}
$$

Na estimativa acima, estamos utilizando que $\sigma_{\lambda}\left(P_{\lambda} \phi_{\lambda}\right)=\phi_{\lambda}$.

Por (J2) e $x \in B_{\bar{\eta}}\left(\phi_{0}\right)$ então:

$$
d\left(y, V_{\lambda}\right) \leq \bar{\eta}+M q \bar{\eta}+2 M p L-\sigma \bar{\eta}+M \bar{\eta} \leq \eta .
$$

OBSERVAÇÃO: Se no teorema anterior tivermos dois parâmetros $\lambda$ e $n$ e desejarmos analisar a semi-continuidade inferior das variedades instáveis locais relativamente a um parâmetro, $\lambda$, mas uniforme no segundo parâmetro, $n$, então a uniformidade das hipóteses do teorema relativamente ao segundo parâmetro não é suficiente para garantir o resultado pois, como pode ser observado na demonstração, a escolha de $\lambda_{1}$ e consequentemente de $\lambda$, depende de $\tau$. Se $\tau$ puder ser escolhido independente de $n$, então o resultado é assegurado uniformemente em $n$.

O teorema a seguir nos dá condições suficientes para obtermos as hipóteses (J4), (J5) e (J6), no caso da família de semigrupos ser gerada pela família de equações do tipo

$$
\dot{x}(t)=L_{\lambda} x+f_{\lambda}(x)
$$

tal que $f(0)=0$ e $d f(0)=0$ (estamos considerando o equilíbrio trival).

Teorema 1.12. Suponhamos que as seguintes hipóteses estão satisfeitas

(K1) Existe um $N$ tal que a constante de Lipschitz de $f_{\lambda}$ em $B_{1 / n}(0)$ pode ser escolhida independente de $\lambda$;

(K2) Existem $\gamma^{+}>0$ e $\gamma^{-}<0$ tais que $\sigma\left(L_{\lambda}\right) \bigcap\left[\gamma^{-}, \gamma^{+}\right]=\emptyset$; 
Agora, definimos

$$
g_{n}(x, y)= \begin{cases}g(x, y), & \|x+y\| \leq \frac{1}{n} \\ g\left(\frac{x}{n\|x+y\|}, \frac{y}{n\|x+y\|}\right), & \|x+y\|>\frac{1}{n}\end{cases}
$$

e

$$
h_{n}(x, y)= \begin{cases}h(x, y), & \|x+y\| \leq \frac{1}{n} \\ h\left(\frac{x}{n\|x+y\|},\right. & \left.\frac{y}{n\|x+y\|}\right)\|x+y\|>\frac{1}{n}\end{cases}
$$

E as constantes de Lispchitz de $g_{n}$ e $h_{n}$ são as constantes de Lipschitz de $g$ e $h$ na bola de raio $\frac{1}{n}$ e assim, $L_{g_{n}}$ e $L_{h_{n}}$ convergem para 0 quando $n \rightarrow \infty$.

Nós denotaremos por $M_{g_{n}}$ e $M_{h_{n}}$ o máximo de $g_{n}$ e $h_{n}$ respectivamente. Temos que $M_{g_{n}} \rightarrow 0$ e $M_{h_{n}} \rightarrow 0$ quando $n \rightarrow 0$. Definamos a classe de funções:

$$
C_{D, \delta}=\left\{\sigma: U \rightarrow S:\|\sigma\|\left\|:=\sup _{y \in U}\right\| \sigma(y) \| \leq D \text { e }\left\|\sigma(y)-\sigma\left(y^{\prime}\right)\right\| \leq \delta\left\|y-y^{\prime}\right\|\right\}
$$

Vamos procurar um ponto fixo nesta classe $\mathcal{C}_{D, \delta}$.

Fixemos $\tau \in \mathbb{R}, \eta \in U$ e $\sigma \in \mathcal{C}$ e definamos:

$$
\begin{aligned}
& y(t)=T_{+}(t-\tau) y(\tau)+\int_{\tau}^{t} T_{+}(t-s) h_{n}(\sigma(x(s)), y(s)) d s \\
& y(\tau)=\eta
\end{aligned}
$$

para $t \leq \tau, y(t)$ é solução de $\frac{d}{d t} y=A y+h_{n}(\sigma(x), y), y \in U$. Desde que $U$ é de dimensão finita e $y(t)$ é limitada para todo $t$, então $y(t)$ existe para todo $t<0$. Nós podemos definir a aplicação $G(\sigma)(\eta): U \rightarrow S$ como

$$
G(\sigma)(\eta)=\int_{-\infty}^{\tau} T_{-}(\tau-s) g_{n}(\sigma(y(s)), y(s)) d s
$$

Observamos que $P G(\sigma)(\eta)=0$ e assim, $G(\sigma)(\eta) \in S$.

Primeiramente vemos que $G(\sigma)(\eta)$ é limitada em $U$, para isso usamos as estimativas (1.12),

$$
\|G(\sigma)(\eta)\| \leq \int_{-\infty}^{\tau} K e^{-\beta(\tau-s)} M_{g_{n}} d s=\frac{K M_{g_{n}}}{\beta}
$$

Desde que $M_{g_{n}} \rightarrow 0$ quando $n \rightarrow \infty$ então escolha $n_{1}$ suficientemente grande tal que $\frac{K M_{g_{n}}}{\beta} \leq D$ para todo $n \rightarrow n_{1}$. Agora mostraremos que $G(\sigma)(\eta)$ é Lipschtiz em $\eta$ e $G$ é 
(K3) Existem $K>1, \beta>0$ e $\rho>0$, independentes de $\lambda$, tais que

$$
\left\|T_{\lambda}(t) x\right\| \leq K e^{\rho t}\|x\|, t \leq 0, x \in U
$$

$e$

$$
\left\|T_{\lambda}(t) x\right\| \leq K e^{-\beta t}\|x\|, t \geq 0, x \in S
$$

então, fixado $j$, para $\phi_{j, \lambda}$ existe um $\delta$ tal que para todo $\lambda, W_{\lambda} \cap B_{\delta}\left(\phi_{\lambda}\right)$ é homeomorfo a $U_{\lambda} \cap B_{\delta}\left(\phi_{\lambda}\right)$ e, enquanto $U_{\lambda}(t) \in B_{\delta}\left(\phi_{\lambda}\right)$ temos:

$$
\left\|Q_{j \lambda} x_{\lambda}(t)-\sigma_{j \lambda}\left(P_{j \lambda} x_{\lambda}(t)\right)\right\| \leq M e^{-\frac{\beta}{2} t}\left\|Q_{j \lambda} u_{0}-\sigma_{j \lambda}\left(P_{j \lambda} u_{0}\right)\right\|,
$$

e as constantes de Lipschitz $L_{\sigma}$ dos homeomorfismo podem ser escolhidas uniformem " em $\lambda$.

\section{Demonstração:}

Fixamos um valor do parâmetro e mostraremos o resultado para este valor fixado. No final da demonstração fazemos as considerações necessárias para a uniformidade em $\lambda$. Nesta demonstração não utilizaremos o índice $\lambda$. Para a demonstração do resultado, consideramos a seguinte sequência de problemas

$$
\begin{aligned}
& x(t)=T_{-}(t-\tau) x(\tau)+\int_{\tau}^{t} T_{-}(t-s) g_{n}(x(s), y(s)) d s \\
& y(t)=T_{+}(t-\tau) y(\tau)+\int_{\tau}^{t} T_{+}(t-s) h_{n}(x(s), y(s)) d s
\end{aligned}
$$

onde para cada $n$, as funções $g_{n}$ e $h_{n}$ são globalmente Lipschitz e suas constantes de Lipschitz convergem para 0 quando $n \rightarrow \infty$. Mostraremos que para $n$ suficientemente grande existe uma variedade instável global $H^{*}=\{\phi \in X ;\|T(t) \phi\|$ existe e é limitado para todo $t \leq 0\}$, onde $W$ é invariante, globalmente homeomorfo a $U$ e globalmente atrator.

Para obtermos a sequência acima da equação original, fazemos o seguinte processo. Denotamos por $g(x(s), y(s))$ a projeção de $f(x(s), y(s))$ sobre a variedade instável do problema linearizado, ou seja, $g(x(s), y(s))=P f(x(s), y(s))$ e por $h(x(s), y(s))$ a projeção $Q f(x(s), y(s))$. Entāo $g(0,0)=0, h(0,0)=0, D g(0,0)=0$ e $D h(0,0)=0$ e

$$
\begin{aligned}
& x(t)=T_{-}(t-\tau) x(\tau)+\int_{\tau}^{t} T_{-}(t-s) g(x(s): y(s)) d s \\
& y(t)=T_{+}(t-\tau) y(\tau)+\int_{\tau}^{t} T_{+}(t-s) h(x(s) ; y(s)) d s
\end{aligned}
$$


uma contração sobre $\mathcal{C}$. Denotemos por $y^{\prime}(t), y(t) \operatorname{com} \sigma$ e $\eta$ trocados por $\sigma^{\prime}$ e $\eta^{\prime}$, então,

$$
\begin{aligned}
\| y^{\prime}(t) & -y(t)\|\leq\| T_{+}(t-\tau) \eta-T_{+}(t-\tau) \eta^{\prime} \| \\
& +\left\|T_{+}(t-\tau)\left(h_{n}(\sigma(y(s)), y(s))-h_{n}\left(\sigma^{\prime}\left(y^{\prime}(s)\right), y^{\prime}(s)\right)\right) d s\right\| \\
& \leq K e^{\rho}(t-\tau)\left\|\eta-\eta^{\prime}\right\|+K \int_{t}^{\tau} e^{\rho(t-s)}\left\|h_{n}(\sigma(y(s)), y(s))-h_{n}\left(\sigma^{\prime}\left(y^{\prime}(s)\right), y^{\prime}(s)\right)\right\| d s \\
& \leq K e^{\rho}(t-\tau)\left\|\eta-\eta^{\prime}\right\|+K \int_{t}^{\tau} e^{\rho(t-s)} L_{h_{n}}\left(\left\|\sigma(y(s))-\sigma^{\prime}\left(y^{\prime}(s)\right)\right\|+\left\|y(s)-y^{\prime}(s)\right\|\right) d s \\
& \leq K e^{\rho}(t-\tau)\left\|\eta-\eta^{\prime}\right\|+K L_{h_{n}} \int_{t}^{\tau} e^{\rho(t-s)}\left(\left\|\sigma-\sigma^{\prime}\right\|+(1+\delta)\left\|y(s)-y^{\prime}(s)\right\|\right) d s \\
& \leq K e^{\rho}(t-\tau)\left\|\eta-\eta^{\prime}\right\|+\frac{K L_{h_{n}}}{\rho}\left\|\sigma-\sigma^{\prime}\right\|\left(1-e^{\rho(t-\tau)}\right) \\
& +K L_{h_{n}} \int_{t}^{\tau} e^{\rho(t-s)}(1+\delta)\left\|y(s)-y^{\prime}(s)\right\| d s
\end{aligned}
$$

Pela desigualdade do lema (1.3), temos

$$
\left\|y^{\prime}(t)-y(t)\right\| \leq K\left(\left\|\eta-\eta^{\prime}\right\|+\frac{K L_{h_{n}}}{\rho}\left\|\sigma-\sigma^{\prime}\right\| \|\right) e^{-\left(\rho-K L_{h_{n}}(1+\delta)\right)(t-\tau)}
$$

Vamos estimar $\left\|G(\sigma)(\eta)-G\left(\sigma^{\prime}\right)\left(\eta^{\prime}\right)\right\|$

$$
\begin{aligned}
\left\|G(\sigma)(\eta)-G\left(\sigma^{\prime}\right)\left(\eta^{\prime}\right)\right\| & =\left\|\int_{-\infty}^{\tau} T_{-}(\tau-s)\left(g_{n}\left(\sigma^{\prime}\left(y^{\prime}(s)\right), y^{\prime}(s)\right)-g_{n}(\sigma(y(s)), y(s))\right) d s\right\| \\
& \leq K L_{g_{n}} \int_{-\infty}^{\tau} e^{-\beta(\tau-s)}\left(\left\|\sigma^{\prime}\left(y^{\prime}(s)\right)-\sigma(y(s))\right\|+\left\|y^{\prime}(s)-y(s)\right\|\right) d s \\
& \leq K L_{g_{n}} \int_{-\infty}^{\tau} e^{-\beta(\tau-s)}\left(\left\|\sigma^{\prime}-\sigma\right\|+(1+\delta)\left\|y^{\prime}(s)-y(s)\right\|\right) d s \\
& \leq I_{1}\left\|\eta-\eta^{\prime}\right\|+I_{2}\left\|\sigma-\sigma^{\prime}\right\|
\end{aligned}
$$

onde

$$
\begin{aligned}
I_{1} & =K^{2} L_{g_{n}}(1+\delta) \int_{-\infty}^{\tau} e^{-\beta(\tau-s)} e^{-\left(\rho-K L_{h_{n}}(1+\delta)\right)(\tau-s)} d s \\
& =\frac{K^{2} L_{g_{n}}(1+\delta)}{\beta+\rho-K L_{h_{n}}(1+\delta)}
\end{aligned}
$$

e

$$
\begin{aligned}
I_{2} & =K L_{g_{n}} \int_{-\infty}^{\tau} e^{-\beta(\tau-s)}\left(1+\frac{K L_{g_{n}}}{\rho}(1+\delta) e^{-\left(\rho-K L_{h_{n}}(1+\delta)\right)(\tau-s)}\right) d s \\
& =K L_{g_{n}} \int_{-\infty}^{\tau} e^{-\beta(\tau-s)} d s+\frac{K^{2} L_{g_{n}}^{2}}{\rho}(1+\delta) \int_{-\infty}^{\tau} e^{-\beta(\tau-s)} e^{-\left(\rho-K L_{h_{n}}(1+\delta)\right)(\tau-s)} d s \\
& =\frac{K L_{g_{n}}}{\beta}+\frac{K^{2} L_{g_{n}}^{2}(1+\delta)}{\rho\left(\beta+\rho-K L_{h_{n}}(1+\delta)\right)}
\end{aligned}
$$


Desde que $L_{g_{n}}, L_{h_{n}}$ vai para 0 quando $n \rightarrow \infty$ então escolhemos $n_{2}$ suficientemente grande tal que

$$
\left\|G\left(\sigma^{\prime}\right)\left(\eta^{\prime}\right)-G(\sigma)(\eta)\right\| \leq \delta\left\|\eta-\eta^{\prime}\right\|+\theta\left\|\sigma-\sigma^{\prime}\right\|
$$

onde $\theta<1$. Portanto, para $n \geq \max \left\{n_{1}, n_{2}\right\}$ temos que $G$ tem um ponto fixo em $\mathcal{C}$. Denotaremos este ponto fixo por $\sigma_{n}$ e assim, $\sigma_{n}(\eta)=G\left(\sigma_{n}\right)(\eta)$. Este homeomorfismo nos dá a variedade $W_{n}=\left\{\left(\sigma_{n}(y), y\right): y \in U\right\}$ resta provar que esta variedade é a variedade instável, ou seja, que $W_{n}$ é invariante e que as soluções em $W_{n}$ são exatamente as soluções que permanecem limitadas quando $t \rightarrow-\infty$.

Seja $\phi$ uma condição inicial em $W_{n}$. Então $\phi=\left(\sigma_{n}(P \phi), P \phi\right)$. Uma solução de (1.82) através de $\phi \dot{e}$

$$
u(t)=T_{+}(t) P \phi+\int_{-\infty}^{\tau} T_{-}(t-s) g_{n}(x(s), y(s)) d s+\int_{0}^{t} T_{+}(t-s) h_{n}(x(s), y(s)) d s .
$$

Observamos que para $t=0$ temos

$$
u(0)=P \phi+\int_{-\infty}^{0} T_{-}(t-s) g_{n}(x(s), y(s)) d s=P \phi+\sigma_{n}(P \phi)=\phi
$$

Por (1.12) sobre $U$, temos que $u(t)$ é limitada quando $t \rightarrow-\infty$. A componente de $u(t)$ em $S$ é dada por:

$$
\begin{aligned}
x(t) & =Q \int_{-\infty}^{\tau} T_{-}(t-s) g_{n}(x(s), y(s)) d s \\
& =\int_{-\infty}^{\tau} T_{-}(t-s) g_{n}(x(s), y(s)) d s=G(\sigma)(y(t))=\sigma(y(t))
\end{aligned}
$$

Logo, $u(t) \in W_{n}$.

Agora vamos mostrar que a variedade instável é exponencialmente atratora; ou seja,

$$
\| Q u(t)-\sigma\left(P(u(t))\left\|\leq K e^{-\frac{\beta}{2}}\right\| Q u(0)-\sigma_{n}(P u(0)) \| .\right.
$$

Assim, seja $u(t)=(x(t), y(t))$ uma solução de (1.82), para $n \geq N$. Desde que desejamos estimar $\| Q u(t)-\sigma\left(P(u(t)) \|\right.$, definimos $\xi(t)=x(t)-\sigma_{n}(y(t))$ e vamos estimar $\xi(t)$.

Definimos

$$
y^{*}(s, t)=T_{+}(s-t) y(t)+\int_{t}^{s} T_{+}(s-\theta) h_{n}\left(\sigma_{n}\left(y^{*}(\theta \cdot t)\right) \cdot y^{*}(\theta \cdot t)\right) d \theta
$$


Observamos que $y^{*}(t, t)=y(t)$ e nós temos

$$
\begin{aligned}
\left\|y^{*}(s, t)-y(s)\right\| & =\| T_{+}(s-t) y(t)+\int_{t}^{s} T_{+}(s-\theta) h_{n}\left(\sigma_{n}\left(y^{*}(\theta, t)\right), y^{*}(\theta, t)\right) d \theta \\
& -T_{+}(s-t) y(t)-\int_{t}^{s} T_{+}(s-\theta) h_{n}(x(\theta), y(\theta)) d \theta \| \\
& \leq \int_{t}^{s} K e^{\rho(s-\theta)}\left\|h_{n}\left(\sigma_{n}\left(y^{*}(\theta, t)\right), y^{*}(\theta, t)\right)-h_{n}(x(\theta), y(\theta))\right\| d \theta \\
& \leq K L_{h_{n}} \int_{t}^{s} e^{\rho(s-\theta)}\left(\left\|\sigma_{n}\left(y^{*}(\theta, t)\right)-x(\theta)\right\|-\left\|y^{*}(\theta, t)-y(\theta)\right\|\right) d \theta \\
& \leq K L_{h_{n}} \int_{t}^{s} e^{\rho(s-\theta)}\left(\left\|\sigma_{n}\left(y^{*}(\theta, t)\right)-\sigma_{n}(y(\theta))\right\|+\left\|\sigma_{n}(y(\theta))-x(\theta)\right\|\right. \\
& \left.-\left\|y^{*}(\theta, t)-y(\theta)\right\|\right) d \theta \leq K L_{h_{n}}(1+\delta) \int_{t}^{s} e^{\rho(s-\theta)}\left\|y^{*}(\theta, t)-y(\theta)\right\| d \theta \\
& +K L_{h_{n}} \int_{s}^{t} e^{\rho(s-\theta)}\|\xi(\theta)\| d \theta
\end{aligned}
$$

Assim, pela desigualdade do lema (1.3), temos

$$
\left\|y^{*}(s, t)-y(s)\right\| \leq K L_{h_{n}} \int_{s}^{t} e^{-\left(\rho-K L_{h_{n}}(1+\delta)\right)(\theta-s)}\|\xi(\theta)\| d \theta
$$

Para $s \leq t_{0} \leq t$ temos

$$
\begin{gathered}
\left\|y^{*}(s, t)-y\left(s, t_{0}\right)\right\|=\| T_{+}(s-t) y(t)+\int_{t}^{s} T_{+}(s-\theta) h_{n}\left(\sigma_{n}\left(y^{*}(\theta, t)\right), y^{*}(\theta, t)\right) d \theta \\
\left.-T_{+}\left(s-t_{0}\right) y(t)-\int_{t_{0}}^{s} T_{+}(s-\theta) h_{n}\left(\sigma_{n}\left(y^{*}\left(\theta, t_{0}\right)\right)\right), y^{*}\left(\theta, t_{0}\right)\right) d \theta \| \\
\leq\left\|T_{+}(s-t) y(t)+\int_{t}^{t_{0}} T_{+}(s-\theta) h_{n}\left(\sigma_{n}\left(y^{*}(\theta, t)\right), y^{*}(\theta, t)\right) d \theta-T_{+}\left(s-t_{0}\right) y(t)\right\| \\
\left.\left.\quad+\int_{t_{0}}^{s} \| T_{+}(s-\theta) h_{n}\left(\sigma_{n}\left(y^{*}(\theta, t)\right)\right), y^{*}(\theta, t)\right)-h_{n}\left(\sigma_{n}\left(y^{*}\left(\theta, t_{0}\right)\right)\right), y^{*}\left(\theta, t_{0}\right)\right) \| d \theta \\
\leq K e^{\rho(s-\theta)}\left\|T_{+}\left(t_{0}-t\right) y(t)+\int_{t}^{t_{0}} T_{+}\left(t_{0}-\theta\right) h_{n}\left(\sigma_{n}\left(y^{*}(\theta, t)\right), y^{*}(\theta, t)\right) d \theta-y\left(t_{0}\right)\right\| \\
\left.\left.\quad+K \int_{t_{0}}^{s} e^{\rho\left(s-t_{0}\right)} \| h_{n}\left(\sigma_{n}\left(y^{*}(\theta, t)\right)\right), y^{*}(\theta, t)\right)-h_{n}\left(\sigma_{n}\left(y^{*}\left(\theta, t_{0}\right)\right)\right), y^{*}\left(\theta, t_{0}\right)\right) \| d \theta \\
\leq K e^{\rho\left(s-t_{0}\right)}\left\|y^{*}\left(t_{0}, t\right)-y\left(t_{0}\right)\right\|+K L_{h_{n}}(1+\delta) \int_{t_{0}}^{s} e^{\rho\left(s-t_{0}\right)}\left\|y^{*}(\theta, t)-y^{*}\left(\theta, t_{0}\right)\right\| d \theta
\end{gathered}
$$

Pela desigualdade do lema (1.3), temos:

$$
\left\|y^{*}(s, t)-y\left(s, t_{0}\right)\right\| \leq K^{2} L_{h_{n}} \int_{t_{0}}^{t} e^{-\left(\rho-K L_{h_{n}}(1+\delta)\right)(\theta-s)}\|\xi(\theta)\| d \theta
$$


Definimos $z(t):=\left\|\xi(t)-T_{-}\left(t-t_{0}\right) \xi\left(t_{0}\right)\right\|$

$$
\begin{aligned}
z(t)= & \left\|x(t)-\sigma_{n}(y(t))-T_{-}\left(t-t_{0}\right)\left(x\left(t_{0}\right)-\sigma_{n}\left(y\left(t_{0}\right)\right)\right)\right\| \\
= & \| T_{-}\left(t-t_{0}\right) x\left(t_{0}\right)+\int_{t_{0}}^{t} T_{-}(t-\tau) g_{n}(x(\tau), y(\tau)) d \tau \\
& \quad-\sigma_{n}(y(t))-T_{-}\left(t-t_{0}\right) x\left(t_{0}\right)+T_{-}\left(t-t_{0}\right) \sigma_{n}\left(y\left(t_{0}\right)\right) \| \\
= & \| \int_{t_{0}}^{t} T_{-}(t-\tau) g_{n}(x(\tau), y(\tau)) d \tau-\int_{-\infty}^{t} T_{-}(t-\tau) g_{n}\left(\sigma_{n}\left(y^{*}(\tau, t)\right), y^{*}(\tau, t)\right) d \tau \\
& \quad+T_{-}\left(t-t_{0}\right) \int_{-\infty}^{t_{0}} T_{-}\left(t_{0}-\tau\right) g_{n}\left(\sigma_{n}\left(y^{*}\left(\tau, t_{0}\right)\right), y^{*}\left(\tau, t_{0}\right)\right) d \tau \| \\
=\| \int_{t_{0}}^{t} T_{-}(t-\tau)\left(g_{n}(x(\tau), y(\tau))-g_{n}\left(\sigma_{n}\left(y^{*}(\tau, t)\right), y^{*}(\tau, t)\right)\right) d \tau & \quad+\int_{-\infty}^{t_{0}} T_{-}(t-\tau)\left(g_{n}\left(\sigma_{n}\left(y^{*}(\tau, t)\right), y^{*}(\tau, t)\right)-g_{n}\left(\sigma_{n}\left(y^{*}\left(\tau, t_{0}\right)\right), y^{*}\left(\tau, t_{0}\right)\right)\right) d \tau \|
\end{aligned}
$$

Por (1.12) temos

$$
\begin{aligned}
z(t) \leq & K L_{g_{n}} \int_{t_{0}}^{t} e^{-\beta(t-\tau)}\left(\left\|x(\tau)-\sigma_{n}\left(y^{*}(\tau, t)\right)\right\| d \tau+\left\|y(\tau)-y^{*}(\tau, t)\right\|\right) d \tau \\
& +K L_{g_{n}} \int_{-\infty}^{t_{0}} e^{-\beta(t-\tau)}\left(\left\|\sigma_{n}\left(y^{*}(\tau, t)\right)-\sigma_{n}\left(y^{*}\left(\tau, t_{0}\right)\right)\right\|+\left\|y(\tau, t)-y^{*}\left(\tau, t_{0}\right)\right\|\right) d \tau \\
\leq & \left.K L_{g_{n}} \int_{t_{0}}^{t} e^{-\beta(t-\tau)}\|\xi(\tau)\| d \tau+K L_{g_{n}}(1+\delta) \int_{t_{0}}^{t} e^{-\beta(t-\tau)}\left\|y(\tau)-y^{*}(\tau, t)\right\|\right) d \tau \\
& +K L_{g_{n}}(1+\delta) \int_{-\infty}^{t_{0}} e^{-\beta(t-\tau)}\left(\left\|y^{*}(\tau, t)-y^{*}\left(\tau, t_{0}\right)\right\| d \tau\right. \\
=K & L_{g_{n}} \int_{t_{0}}^{t} e^{-\beta(t-\tau)}\|\xi(\tau)\| d \tau \\
& \left.+K L_{g_{n}}(1+\delta) \int_{t_{0}}^{t} e^{-\beta(t-\tau)}\right) K L_{h_{n}} \int_{\tau}^{t} e^{-\left(\rho-K L_{h_{n}}(1+\delta)\right)(\theta-\tau)}\|\xi(\theta)\| d \theta d \tau \\
& +K L_{g_{n}}(1+\delta) \int_{-\infty}^{t_{0}} e^{-\beta(t-\tau)} K^{2} L_{h_{n}} \int_{t_{0}}^{t} e^{-\left(\rho-K L_{h_{n}}(1+\delta)\right)(\theta-\tau)}\|\xi(\theta)\| d \theta d \tau \\
\leq \quad & K L_{g_{n}} \int_{t_{0}}^{t} e^{-\beta(t-\tau)}\|\xi(\tau)\| d \tau \\
& +K^{3} L_{g_{n}} L_{h_{n}}(1+\delta) \int_{t_{0}}^{t} \int_{t_{0}}^{\theta} e^{-\beta(t-\tau)} e^{-\left(\rho-K L_{h_{n}}(1+\delta)\right)(\theta-\tau)}\|\xi(\theta)\| d \tau d \theta \\
& +K^{3} L_{g_{n}} L_{h_{n}}(1+\delta) \int_{t_{0}}^{t} \int_{-\infty}^{t_{0}} e^{-\beta(t-\tau)} e^{-\left(\rho-K L_{h_{n}}(1+\delta)\right)(\theta-\tau)}\|\xi(\theta)\| d \tau d \theta
\end{aligned}
$$


e assim,

$$
\begin{aligned}
z(t) & \leq K L_{g_{n}} \int_{t_{0}}^{t} e^{-\beta(t-\tau)}\|\xi(\tau)\| d \tau \\
& +K^{3} L_{g_{n}} L_{h_{n}}(1+\delta) \int_{t_{0}}^{t} \int_{-\infty}^{\theta} e^{-\beta(t-\tau)} e^{-\left(\rho-K L_{h_{n}}(1+\delta)\right)(\theta-\tau)}\|\xi(\theta)\| d \tau d \theta \\
& =K L_{g_{n}} \int_{t_{0}}^{t} e^{-\beta(t-\tau)}\|\xi(\tau)\| d \tau \\
& +\frac{K^{3} L_{g_{n}} L_{h_{n}}(1+\delta)}{\beta+\rho-K L_{h_{n}}(1+\delta)} \int_{t_{0}}^{t} e^{-\beta t} e^{-\left(\rho-K L_{h_{n}}(1+\delta)\right)(\theta)}\|\xi(\theta)\| e^{\left(\beta+\rho-K L_{h_{n}}(1+\delta)\right)(\theta)} d \theta
\end{aligned}
$$

onde $\beta+\rho-K L_{h_{n}}(1+\delta)>0$ para $n$ suficientemente grande.

Assim,

$$
\begin{aligned}
z(t) & \leq K L_{g_{n}} \int_{t_{0}}^{t} e^{-\beta(t-\tau)}\|\xi(\tau)\| d \tau+\frac{K^{3} L_{g_{n}} L_{h_{n}}(1+\delta)}{\beta+\rho-K L_{h_{n}}(1+\delta)} \int_{t_{0}}^{t} e^{-\beta(t-\theta)}\|\xi(\theta)\| d \theta \\
& \leq \tilde{K} \int_{t_{0}}^{t} e^{-\beta(t-\theta)}\|\xi(\theta)\| d \theta
\end{aligned}
$$

onde

$$
\bar{K}=\frac{\left(K^{3}-K^{2}\right) L_{g_{n}} L_{h_{n}}(1+\delta)+(\beta-\rho) K L_{g_{n}}}{\beta+\rho-K L_{h_{n}}(1+\delta)} .
$$

Agora podemos estimar $\xi(t)$

$$
\|\xi(t)\| \leq \tilde{K} \int_{t_{0}}^{t} e^{-\beta(t-\theta)}\|\xi(\theta)\| d \theta+\left\|T_{-}(t-\tau) \xi\left(t_{0}\right)\right\|
$$

E pela desigualdade do lema (1.3) temos:

$$
\|\xi(t)\| \leq K e^{(\bar{K}-\beta)\left(t-t_{0}\right)}\left\|\xi\left(t_{0}\right)\right\|
$$

e assim escolhemos $\bar{N}$ tal que $\tilde{K} \leq \frac{\beta}{2}$.

Pela demonstração dada e pelo fato que hipóteses (K1), (K2) e (K3) são uniformes em $\lambda$ então o resultado é obtido uniforme em $\lambda$.

Lema 1.3. Sejam a e b funções contínuas a valores reais e suponha que

$$
b(t) \leq L \int_{t}^{\tau} b(\theta) d \theta+a(t), \text { para } 0 \leq t \leq \tau
$$

Então

$$
b(t) \leq L \int_{t}^{\tau} a(\theta) e^{L(\theta-t)} d \theta+a(t) .
$$

Além disso: se a(t) é uma função decrescente então

$$
b(t) \leq a(\tau) e^{L(\tau-t)}-\int_{t}^{\tau} \dot{a}(\theta) e^{L(\theta-t)} d \theta \leq a(t) e^{L(\tau-t)}
$$




\section{Capítulo 2}

\section{Problema Parabólico}

Neste capítulo vamos mostrar a equivalência topológica dos semigrupos sobre os atratores dos problemas

$$
\begin{aligned}
& u_{t}=u_{x x}+f(u), \quad 0<x<1, t>0 \\
& u_{x}(0)=u_{x}(1)=0, t>0,
\end{aligned}
$$

e

$$
\dot{U}=-\triangle_{p} U+\mathrm{f}(U)
$$

onde $f: \mathbb{R} \rightarrow \mathbb{R}$ é uma função de classe $C^{2}$ satisfazendo a condição de dissipatividade (1.3), $\Delta_{p}$ é a matriz a $p \times p$ dada por $(1.10)$ e $\mathrm{f}(U)=\left(f\left(u_{1}\right)_{:} \cdots, f\left(u_{p}\right)\right)^{\top}$.

Os resultados do capítulo 1 , nos garantem a existência de um atrator global $\mathcal{A}$ para (2.1) e um atrator global $\mathcal{A}_{p}$ para $(2.2)$.

A equivalência topológica de $\mathcal{A}$ e $\mathcal{A}_{p}$ é obtida quando o tamanho do passo, $p^{-1}$, utilizado na discretização do problema (2.1) é suficientemente pequeno, ou seja, $p$ é suficientemente grande.

Como foi comentado na introdução, foi necessário definir em $\mathbb{R}^{p}$ um produto interno compatível com o produto interno $L^{2}$.

Sejam $\lambda_{1}, \ldots, \lambda_{n}, \ldots$ os autovalores e $\phi_{1}, \ldots, \phi_{n}, \ldots$ as respectivas autofunçōes de $-u_{x x}$ com condição de fronteira de Neumann. $\mathrm{E} \lambda_{1}^{p}, \ldots, \lambda_{n}^{p}$ os autovalores e $\phi_{1}^{p}, \ldots, \phi_{n}^{p}$ os respectivos autovetores de $\Delta_{p}$. Então, se (2.1) possui uma variedade invariante exponencialmente atratora dada por $\sigma_{n}: W=\left[\phi_{1}, \ldots, \phi_{n}\right] \rightarrow W^{-1}$ a equação (2.1) reduz-se 


$$
\dot{v}=-\left[\begin{array}{ccccc}
\lambda_{1} & 0 & 0 & \ldots & 0 \\
0 & \lambda_{2} & 0 & \ldots & 0 \\
\vdots & \vdots & \ddots & \vdots & \vdots \\
0 & 0 & \cdots & \lambda_{n-1} & 0 \\
0 & 0 & \cdots & 0 & \lambda_{n}
\end{array}\right] v+g_{n}\left(v, \sigma_{n}(v)\right)
$$

e após uma mudança de coordenadas (2.2) torna-se

$$
\dot{v}=-\left[\begin{array}{ccccc}
\lambda_{1}^{p} & 0 & 0 & \cdots & 0 \\
0 & \lambda_{2}^{p} & 0 & \cdots & 0 \\
\vdots & \vdots & \ddots & \vdots & \vdots \\
0 & 0 & \cdots & \lambda_{p-1}^{p} & 0 \\
0 & 0 & \cdots & 0 & \lambda_{p}^{p}
\end{array}\right] v+F(v)
$$

Se $n=p$ os problemas acima possuem o mesmo espaço de fase. Como $\lambda_{i}^{p}-\lambda_{i}$ não pode ser feito pequeno para todo $i$, então não podemos esperar obter que as dinâmicas assintóticas são suficientemente próximas. Ao fazer $p \gg n$ temos que $\sup _{1 \leq i \leq n}\left|\lambda_{i}^{p}-\lambda_{i}\right|$ se aproxima de zero. Neste caso (2.3) e (2.4) não possuem mais o mesmo espaço de fase. Projetamos (2.4) sobre uma variedade invariante exponencialmente atratora $n$ dimensional dada pelo gráfico de

$$
\sigma_{n}^{p}: W_{n}^{p}=\left[\phi_{1}^{p}, \phi_{2}^{p}, \ldots, \phi_{n}^{p}\right] \rightarrow\left(W_{n}^{p p}\right)^{\perp}
$$

e (2.4) fica reduzida a

$$
\dot{Z}=-\left[\begin{array}{ccccc}
\lambda_{1}^{p} & 0 & 0 & \cdots & 0 \\
0 & \lambda_{2}^{p} & 0 & \cdots & 0 \\
\vdots & \vdots & \ddots & \vdots & \vdots \\
0 & 0 & \cdots & \lambda_{n-1}^{p} & 0 \\
0 & 0 & \cdots & 0 & \lambda_{n}^{p}
\end{array}\right] Z+\tilde{F}\left(Z, \sigma_{n}^{p}(Z)\right)
$$

Agora estamos em condições de realizar a comparação dos campos. Se $p \gg n$ temos que $\sup _{1 \leq i \leq n}\left|\lambda_{i}^{p}-\lambda_{i}\right|$ é pequeno e por outro lado. se $p \gg n \gg 1$ temos que $\sigma_{n}^{p}$ e $\sigma_{n}$ são $C^{\mathbf{l}}$ próximas de zero e portanto negligenciáveis.

Este é o argumento que empregaremos para mostrar que as dinâmicas assintóticas de (2.1) e (2.2) são equivalentes. 


\subsection{Discretização}

Primeiramente nós discutimos a discretização espacial de (2.1). Consideramos os pontos $x_{j}=\frac{j-\frac{1}{2}}{p}, j=1, \cdots, p$ e denotamos $u_{j}(t)=u\left(x_{j}, t\right)$. Então, nós temos

$$
\begin{aligned}
& \dot{u}_{1}=p^{2}\left(u_{2}-u_{1}\right)+f\left(u_{1}\right) \\
& \dot{u}_{j}=p^{2}\left(u_{j-1}-2 u_{j}+u_{j+1}\right)+f\left(u_{j}\right), \quad j=2, \cdots, p-1 \\
& \dot{u}_{p}=p^{2}\left(u_{p-1}-u_{p}\right)+f\left(u_{p}\right)
\end{aligned}
$$

Observamos que as condições de fronteira, pela discretização, se transformam em $u_{1}=$ $u_{0}, u_{p+1}=u_{p}$ e já foram incorporadas nas equaçōes (2.6).

Denotando $U=\left(u_{1}, \cdots, u_{p}\right)^{\top}$ e reescrevendo a equação acima na forma matricial, nós obtemos:

$$
\dot{U}=-\Delta_{p} U+\mathrm{f}(U)
$$

onde $\Delta_{p}$ é a matriz a $p \times p$ dada por $(1.10)$ e $f(U)=\left(f\left(u_{1}\right), \cdots, f\left(u_{p}\right)\right)^{\top}$.

Nós observamos que o sistema (2.7) é genericamente Morse-Smale (ver [FO]).

A condição de dissipatividade (1.3) implica que existe $\xi>0$ tal que $f(u) u<0$ para $|u| \geq \xi$. Esta última condição é suficiente para obter os resultados da seção 1.2. Por conveniência, vamos manter a condição (1.3). Pelas condições impostas a $f$, o problema acima tem um atrator global $\mathcal{A}_{p}$ que satisfaz

$$
\mathcal{A}_{p} \subset R_{\xi}^{p}
$$

onde $R_{\xi}^{p}=\left\{v \in \mathbb{R}^{p} ;\left|v_{i}\right| \leq \xi, 1 \leq i \leq p\right\}$ e $\xi$ satisfaz a condição dada acima, ver [CDR].

Desde que nós estamos interessados em estudar as soluções do problema acima somente no atrator, nós podemos cortar a não linearidade de tal forma que $f$ é limitada e suas derivadas até segunda ordem também são limitadas.

Sabemos, pelo teorema 1.3, que $\lambda_{k}^{p}=4 p^{2} \operatorname{sen}^{2} \frac{k \pi}{2 p}$ são os autovalores de $\Delta_{p}$ e os autovetores associados são $w_{k}^{p}=\left(\cos k \pi x_{1}, \cdots, \cos k \pi x_{p}\right)$, para $k=0, \cdots, p-1$.

Em $\mathbb{R}^{p}$ definimos um produto interno $L_{d}^{2}$ compatível com o produto interno em $X$, dado por $\langle U, V)=\sum_{i=1}^{p} \frac{1}{p} u_{i} v_{i}$. Normalizando $u_{k}^{p}$ de acordo com este produto interno, nós 
obtemos:

$$
\nu_{k}^{p}=\frac{w_{k}^{p}}{\left\|w_{k}^{p}\right\|}=\frac{\left(\cos k \pi x_{1}, \cdots \cdot \cos k \pi x_{p}\right)}{\sqrt{\frac{1}{p} \sum_{i=1}^{p} \cos ^{2} k \pi x_{i}}}
$$

Se nós escrevemos

$$
\nu_{k}^{p}(x)=\frac{\sum_{i=1}^{p} \cos k \pi x_{i} \chi_{I_{i}}(x)}{\sqrt{\frac{1}{p} \sum_{i=1}^{p} \cos ^{2} k \pi x_{i}}}
$$

onde nós denotamos por $I_{i}$ o intervalo $\left[\frac{i-1}{p}, \frac{i}{p}\right]$, nós obtemos que $\nu_{k}^{p}(x) \in L^{\infty}(0,1)$ e $\left\|\nu_{k}^{p}(x)-\sqrt{2} \cos k \pi x\right\|_{\infty} \rightarrow 0$ quando $p \rightarrow \infty$.

Consideramos a base de autovetores $\nu_{k}^{p}, 0 \leq k \leq p-1$, em $\mathbb{R}^{p}$. Esta base é ortonormal com respeito ao produto interno $L_{d}^{2}$. Nós consideramos a equação discretizada nestas novas coordenadas, ou seja, se nós escrevemos: $v_{1}=\left\langle U, \nu_{0}^{p}\right\rangle \cdot \cdots: v_{p}=\left\langle U, \nu_{p-1}^{p}\right\rangle$ e $v=$ $\left(v_{1}, \cdots, v_{p}\right)$ nós obtemos:

$$
\dot{v}=-\tilde{\Delta_{p}} v+F(v)
$$

onde $\tilde{\Delta}_{p}$ é a matriz $p \times p$ dada por $\tilde{\Delta}_{p}=\operatorname{diag}\left(\lambda_{0}^{p}, \cdots, \lambda_{p-1}^{p}\right)$ e $F(v)=\left(F_{1}(v), \cdots, F_{p}(v)\right)^{\top}$ com cada $F_{j}(v)$ dado por

$$
F_{j}(v)=\left\langle\mathrm{f}(U), \nu_{j-1}^{p}\right\rangle=\sum_{k=1}^{p} \frac{1}{p} \nu_{j-1 k}^{p} f\left(\nu_{0 k}^{p} v_{1}+\cdots+\nu_{(p-1) k}^{p} v_{p}\right) .
$$

onde $\nu_{j k}^{p}$ denota a $k$-ésima coordenada de $\nu_{j}^{p}$. Nós denotamos a matriz de mudança de base por $Z$; e é dada por $z_{k j}=\nu_{(j-1) k}^{p}$ e a matriz $Z^{-1}$ é dada por $(1 / p) Z^{\top}$.

Agora, nós consideramos uma discretização com $p=n^{3}$ pontos e consideramos a seguinte decomposição de $\mathbb{R}^{n^{3}}=\mathbb{R}^{n} \oplus \mathbb{R}^{n^{3}-n}$ onde $\mathbb{R}^{n}=\operatorname{span}\left[\nu_{0}^{n^{3}} \ldots, \nu_{n-1}^{n^{3}}\right]$ e $\mathbb{R}^{n^{3}-n}=$ $\operatorname{span}\left[\nu_{n}^{n^{3}}, \ldots, \nu_{n^{3}-1}^{n^{3}}\right]$. Com esta decomposição, nós obtemos o seguinte sistema fracamente acoplado:

$$
\begin{aligned}
& \dot{v}_{n}+\tilde{B}_{n} v_{n}=\tilde{g}_{n}\left(v_{n}, u_{n}\right) \\
& \dot{w}_{n}+\tilde{A}_{n} w_{n}=\tilde{f}_{n}\left(v_{n} ; w_{n}\right),
\end{aligned}
$$

onde $\tilde{B}_{n}$ é matriz diagonal $n \times n$ dada por $\tilde{B}_{n}=\operatorname{diag}\left(\lambda_{0}^{n^{3}}: \cdots \cdot \lambda_{n-1}^{n^{3}}\right)$. $\tilde{A}_{n}$ é a matriz diagonal $\left(n^{3}-n\right) \times\left(n^{3}-n\right)$ dada por $\tilde{A}_{n}=\operatorname{diag}\left(\lambda_{n}^{n^{3}}, \cdots: \lambda_{n^{3}-1}^{n^{3}}\right): \tilde{g}_{n}\left(v_{n}, w_{n}\right)=\left(F_{1}\left(v_{n}, w_{n}\right), \cdots\right.$, $\left.F_{n}\left(v_{n}, w_{n}\right)\right)^{\top}$ e $\tilde{f}_{n}\left(v_{n}, w_{n}\right)=\left(F_{(n+1)}\left(v_{n}, w_{n}\right), \cdots, F_{n^{3}}\left(v_{n}, u_{n}\right)\right)^{-}$.

Para o sistema fracamente acoplado (2.11) mostramos que existe uma variedade invariante $n$-dimensional exponencialmente atratora; isto é, o seguinte resultado vale: 
Teorema 2.1. Seja $f$ de classe $C^{2}$, limitada com primeira e segunda derivadas limitadas; então, o problema (2.11) para n suficientemente grande, possui uma variedade invariante

$$
M_{n}=\left\{\left(v_{n}, w_{n}\right) \in \mathbb{R}^{n^{3}} \mid w_{n}=\tilde{\sigma}_{n}\left(v_{n}\right)\right\}
$$

a qual é exponencialmente atratora, onde $\tilde{\sigma}_{n}$ é uma função suave, $\tilde{\sigma}_{n}: \mathbb{R}^{n} \rightarrow \mathbb{R}^{n^{3}-n}$ e o fluxo sobre $M_{n}$ é dado por $u(t)=v_{n}(t)+\tilde{\sigma}_{n}\left(v_{n}(t)\right)$ onde $v_{n}(t)$ é solução de

$$
\dot{v}_{n}+\tilde{B}_{n} v_{n}=\tilde{g}_{n}\left(v_{n}, \tilde{\sigma}_{n}\left(v_{n}\right)\right)
$$

Além disso, $s(n)=\|\| \tilde{\sigma}_{n}\|\|=\sup _{v_{n} \in \mathbb{R}^{n}}\left\{\tilde{\sigma}_{n}\left(v_{n}\right)\right\}$ e a constante de Lipschitz, $l(n)$, de $\tilde{\sigma}_{n}$ convergem para zero quando $n \rightarrow \infty$.

Demonstraçāo do Teorema 2.1: Fazendo $\alpha=0$ no teorema (1.9) nós temos: $Y_{n} \times X_{n}$ onde $Y_{n}=\mathbb{R}^{n}$ e $X_{n}=\mathbb{R}^{n^{3}-n}, \tilde{g}_{n}: Y_{n} \times X_{n} \rightarrow Y_{n}$ e $\tilde{f}_{n}: Y_{n} \times X_{n} \rightarrow X_{n}$. Nós fazemos a seguinte distinção relativamente as várias normas usadas aqui, quando o índice da norma é $X_{n}$ ou $Y_{n}$ nós estamos usando a base de autovetores e desta forma a norma é dada por $\|\cdot\|=\left(\sum_{i} x_{i}^{2}\right)^{1 / 2}$, quando o índice da norma é $\mathbb{R}^{k}$ nós estamos utilizando a base canônica e a norma é dada pela norma proveniente do produto interno $L^{2}$ discretizado.

Primeiramente fazemos algumas estimativas necessárias sobre $\tilde{f}_{n}$ e $\tilde{g}_{n}$

$$
\begin{aligned}
\left\|\tilde{g}_{n}\left(v_{n}, w_{n}\right)\right\|_{Y_{n}} & =\left(\sum_{i=1}^{n}\left(F_{i}\left(v_{n}, w_{n}\right)\right)^{2}\right)^{1 / 2} \\
& \leq\left\|\mathrm{f}\left(Z\left(v_{n}, w_{n}\right)^{\top}\right)\right\|_{\mathbb{R}^{n^{3}}}=\left(\sum_{i=1}^{n^{3}} \frac{1}{n^{3}}\left(f_{i}\left(Z\left(v_{n}, w_{n}\right)^{\top}\right)\right)^{2}\right)^{1 / 2} \\
& =\left(\sum_{i=1}^{n^{3}} \frac{1}{n^{3}}\left(f\left(\left[Z\left(v_{n}, w_{n}\right)^{\top}\right]_{i}\right)\right)^{2}\right)^{1 / 2} \leq\left(\sum_{i=1}^{n^{3}} \frac{1}{n^{3}}\|f\|_{\infty}^{2}\right)^{1 / 2}=\|f\|_{\infty}
\end{aligned}
$$

Similarmente, nós obtemos a estimativa

$$
\left\|\tilde{f}_{n}\left(v_{n}, w_{n}\right)\right\|_{x_{n}} \leq\|f\|_{\infty}
$$


Para as constantes de Lipschitz nós temos

$$
\begin{aligned}
\left\|\tilde{g}_{n}\left(v_{n}, w_{n}\right)-\tilde{g}_{n}\left(z_{n}, u_{n}\right)\right\|_{Y_{n}} & =\left(\sum_{i=1}^{n}\left(F_{i}\left(v_{n}, w_{n}\right)-F_{i}\left(z_{n}, u_{n}\right)\right)^{2}\right)^{1 / 2} \\
& \leq\left\|\mathrm{f}\left(Z\left(v_{n}, w_{n}\right)^{\top}\right)-\mathrm{f}\left(Z\left(z_{n}, u_{n}\right)^{\top}\right)\right\|_{\mathbb{P}^{3}{ }^{3}} \\
& =\left(\sum_{i=1}^{n^{3}} \frac{1}{n^{3}}\left(f\left(\left[Z\left(v_{n}, w_{n}\right)^{\top}\right]_{i}\right)-f\left(\left[Z\left(z_{n}, u_{n}\right)^{\top}\right]_{i}\right)\right)^{2}\right)^{1 / 2} \\
& \leq\left(\sum_{i=1}^{n^{3}} \frac{1}{n^{3}} L_{f}^{2}\left(\left[Z\left(v_{n}, w_{n}\right)^{\top}\right]_{i}-\left[Z\left(z_{n}, u_{n}\right)^{\top}\right]_{i}\right)^{2}\right)^{1 / 2} \\
& =\left(\sum_{i=1}^{n^{3}} \frac{1}{n^{3}} L_{f}^{2}\left(\left[Z\left(v_{n}-z_{n}, u_{n}-u_{n}\right)^{\top}\right]_{i}\right)^{2}\right)^{1 / 2} \\
& =L_{f}\left\|Z\left(\left(v_{n}-z_{n}\right),\left(w_{n}-u_{n}\right)\right)^{\top}\right\|_{\mathbb{R}^{n^{3}}} \\
& \leq \sqrt{2} L_{f}\left(\left\|v_{n}-z_{n}\right\|_{Y_{n}}+\left\|u_{n}-u_{n}\right\|_{\lambda_{n}}\right) .
\end{aligned}
$$

onde $L_{f}$ é a constante de Lipschitz da função $f$.

Da mesma forma nós obtemos a estimativa para a constante de Lipschitz de $\tilde{f}_{n}$. Todas as constantes são uniformes em $n$.

As constantes $\beta(n)$ e $\rho(n)$ no teorema 1.9 são: $\beta(n)=\lambda_{n}^{n^{3}}$ e $\rho(n)=\lambda_{n-1}^{n^{3}}$. O que nos dá que $\beta(n) \sim n^{2}$ e $\rho(n) \sim(n-1)^{2}$ quando $n \rightarrow \infty, \operatorname{assim} \beta(n)-\rho(n) \rightarrow \infty$ quando $n \rightarrow \infty$.

Portanto, pelo teorema 1.9, temos que para $n$ suficientemente grande. existe uma variedade invariante

$$
M_{n}=\left\{\left(v_{n}, w_{n}\right) \in \mathbb{R}^{n^{3}} \mid w_{n}=\tilde{\sigma}_{n}\left(v_{n}\right)\right\}
$$

a qual é exponencialmente atratora, onde $\tilde{\sigma}_{n}: \mathbb{R}^{n} \rightarrow \mathbb{R}^{n^{3}-n}$ e o fluxo sobre $M_{n}$ é dado por $u(t)=v_{n}(t)+\tilde{\sigma}_{n}\left(v_{n}(t)\right)$ onde $v_{n}(t)$ é solução de

$$
\dot{v}_{n}+\tilde{B}_{n} v_{n}=\tilde{g}_{n}\left(v_{n}, \tilde{\sigma}_{n}\left(v_{n}\right)\right)
$$

Também, pelo teorema 1.9, temos que $s(n)$ e $l(n)$ convergen para zero quando $n \rightarrow \infty$. Além disso, $\tilde{\sigma}_{n}$ é suave e $\left\|D \tilde{\sigma}_{n}\right\| \rightarrow 0$ quando $n \rightarrow \infty$.

\subsection{O Problema Contínuo}

Agora nós retornamos ao problema (2.1). Seja $X=L^{2}(0,1)$, nós definimos $f^{e}: H^{1}(0,1) \subset$ $X \rightarrow X$ por $f^{e}(\phi)(x)=f(\phi(x))$ e definimos: 


$$
\begin{aligned}
& A: D(A) \subset L^{2}(0,1) \rightarrow L^{2}(0,1) \\
& D(A)=H_{N}^{2}(0,1)=\left\{\phi \in H^{2}(0,1): \phi^{\prime}(0)=\phi^{\prime}(1)=0\right\} \\
& A \phi=-\phi^{\prime \prime} .
\end{aligned}
$$

Assim, reescrevemos o problema (2.1) como:

$$
\begin{aligned}
& \frac{d}{d t} u+A u=f^{e}(u), \\
& u(0)=u_{0}
\end{aligned}
$$

Pelas condiçōes impostas a $f$, obtemos que $f^{e}$ é contínua e Lipschitz em subconjuntos limitados de $H^{1}(0,1)$. Então, o problema acima tem um atrator global $\mathcal{A}$ que satisfaz

$$
\sup _{u \in \mathcal{A}} \sup _{x \in[0,1]}|u(x)| \leq \xi .
$$

A limitação acima nos permite cortar a não linearidade $f$ (sem alterar o atrator) de tal forma que $f$ se torne limitada e suas primeira e segunda derivadas sejam limitadas. Também depois de cortar a não linearidade nós podemos colocar o problema em $L^{2}(0,1)$ mantendo o mesmo atrator. Daqui para frente nós assumimos que $f$ é limitada e tem primeira e segunda derivadas limitadas. Nós também assumimos que o problema está posto em $L^{2}(0,1)$.

Seja $\lambda_{0}<\lambda_{1}<\lambda_{2}<\cdots$ a sequência de autovalores de $A$, onde $\lambda_{k}=(k \pi)^{2}$ e $\phi_{0}, \phi_{1}, \phi_{2}, \cdots$ é a correspondente sequência de autofunçōes normalizadas, $\phi_{k}(x)=$ $\sqrt{2} \cos (k \pi x)$.

Agora considere a seguinte decomposição de $X=W \oplus W^{\perp}$ onde

$$
\begin{aligned}
& W=\operatorname{span}\left[\phi_{0}, \phi_{1}, \cdots, \dot{\phi}_{n-1}\right] \\
& W^{\perp}=\left\{\phi \in X:(\phi, w\rangle=0, \forall w \in W^{\prime}\right\}
\end{aligned}
$$

onde $\langle\cdot, \cdot)$ é o produto interno de $L^{2}(0,1)$.

Então, $u \in L^{2}(0,1)$ pode ser escrita como:

$$
u=v_{1} \phi_{0}+v_{2} \phi_{1}+\cdots+v_{n} \phi_{n-1}+u
$$

onde

$$
\begin{aligned}
v_{i} & =\int_{0}^{1} u(x) \phi_{i-1}(x) d x, i=1, \cdots, n \\
w & =u-\sum_{i=1}^{n} v_{i} \phi_{i-1}
\end{aligned}
$$


Seja $u$ uma solução de (2.15); então, para cada $t$, nós podemos escrever

$$
u(t, x)=v_{1}(t) \phi_{0}(x)+v_{2}(t) \phi_{1}(x)+\cdots+v_{n}(t) \phi_{n-1}(x)+w(t, x)
$$

e

$$
\begin{aligned}
& \dot{v}_{i}=-\lambda_{i-1} v_{i}+\left\langle f(u), \phi_{i-1}\right\rangle \\
& w_{t}+A_{n} w=f(u)-\sum_{i=0}^{n-1}\left\langle f(u), \phi_{i}\right\rangle \phi_{i}
\end{aligned}
$$

onde $\left.A_{n} \operatorname{denota} A\right|_{D(A) \cap W^{\perp}}$.

Escrevendo $v=\left(v_{1}, v_{2}, \cdots, v_{n}\right), u=(v, w)$ e $B_{n}$ a matriz diagonal $n \times n: B_{n}=$ $\operatorname{diag}\left(\lambda_{0}, \lambda_{1}, \cdots . \lambda_{n-1}\right)$ nós obtemos o seguinte sistema:

$$
\begin{aligned}
& \dot{v}+B_{n} v=g_{n}(v, w) \\
& w_{t}+A_{n} w=f_{n}(v, w)
\end{aligned}
$$

onde $g_{n}$ e $f_{n}$ são dadas por: $g_{n}(v, w)=\left(\left\langle f(v, w), \phi_{0}\right\rangle, \cdots,\left\langle f(v, w), \phi_{n-1}\right\rangle\right)^{\top}$ e $f_{n}(v, w)=$ $f(v, w)-\sum_{i=0}^{n-1}\left\langle f(v, w), \phi_{i}\right\rangle \phi_{i}$.

Teorema 2.2. Seja $f \in C^{2}(\mathbb{R}, \mathbb{R})$ limitada com primeira e segunda derivadas limitadas; então, para $n$ suficientemente grande existe uma variedade invariante suave e exponencialmente atratora, $S_{n}$ para (2.19). O fluxo sobre $S_{n}$ é dado por: $u(t, x)=\left(v(t), \sigma_{n}(v(t))\right)$ onde $v$ é solução de

$$
\dot{v}+B_{n} v=g_{n}\left(v, \sigma_{n}(v)\right)
$$

Além disso, $s\langle n)=\|\| \sigma_{n}\|\|=\sup _{v \in W}\left\{\left\|\sigma_{n}(v)\right\|\right\}$ e a constante de Lipschitz, $l(n)$, de $\sigma_{n}$ convergem para zero quando $n \rightarrow \infty$.

Demonstração: Sejam $L_{f^{e}}$ a constante de Lipschitz ${ }^{\alpha^{e}} f^{e}$ e $N_{f^{e}}=\|f\|_{\infty}$. As constantes dadas no teorema 1.9, são $L_{f}=L_{g}=L_{f^{c}}, N_{f}=N_{g}=N_{f^{c}}, \beta(n)=\lambda_{n}, \rho(n)=\lambda_{n-1} \mathrm{e}$ observando que $B(n)-\rho(n)=\pi^{2}(2 n \mp 1)$. Portanto, o teorema segue do teorema 1.9.

$$
\lambda_{n}=(n \pi)^{2}
$$

\section{2:3 Convergência Espectral Uniforme}


Para comparar a dinâmica assintótica do problema discretizado com a dinâmica assintótica do problema contínuo, nós projetamos o primeiro sobre a variedade invariante $\tilde{\sigma}_{n}$ e o segundo sobre a variedade invariante $\sigma_{n}$, somente depois disto somos capazes de comparar suas dinâmicas assintóticas. Isto é realizado comparando os campos vetoriais (agora com mesma dimensão finita). Para comparar os campos vetoriais nós precisamos obter uma forma de comparar $\tilde{B}_{n}$ e $B_{n}$. Isto é alcançado se nós provamos a convergência uniforme (com respeito a $n$ ) dos autovalores e autovetores de $\tilde{B}_{n}$ para os autovalores e autofunçōes $B_{n}$ quando $n \rightarrow \infty$.

Uma outra forma de comparar os campos vetoriais seria projetar ambos os problemas sobre variedades invariantes fixas de mesma dimensão e então estudar a convergência dos campos vetoriais. $O$ que envolveria o estudo da convergência das variedades invariantes e traria complicações técnicas desnecessárias. Esta abordagem tem claro a propriedade que os autovalores e autofunções (um número fixo) converge uniformemente. Aqui nós exploramos o fato que para valores grandes de $n$ as variedades invariantes tem norma $C^{1}$ muito pequena e portanto nós podemos simplesmente negligenciá-las; por outro lado precisamos ter cuidado para garantir a convergência uniforme dos autovalores e autofunções. Esta é a razão porque nós fazemos o corte entre o $n$-ésimo e o $(n+1)$-ésimo autovalor com o problema discreto tendo $n^{3}$ autovalores.

Se nós consideramos a matriz $\Delta_{p} \operatorname{com} p=n^{3}$ nós temos $n^{3}$ autovalores simples e autofunçōes ortonormais para $\Delta_{p}$. É claro que estes autovalores e autofunçõs não convergem uniformemente para os $n^{3}$ primeiros autovalores e autofunções do Laplaciano com condição de Neumann quando $n \rightarrow \infty$. É também claro que qualquer subconjunto finito (fixo) de autovalores de $\Delta_{p}$ converge uniformemente para os correspondentes autovalores do Laplaciano com condição de Neumann quando $n \rightarrow \infty$ e de fato, mais que isso é verdade. Os primeiros $n$ autovalores e autofunções da matriz $n^{3} \times n^{3}, \Delta_{n^{3}}$, convergem uniformemente para os primeiros $n$ autovalores e autofunções do Laplaciano com condição de Neumann quando $n \rightarrow \infty$. E isto nós provaremos nas subseções 2.3 .1 e 2.3.2.

\subsubsection{Convergência Uniforme dos Autovalores}

Os $n$ primeiros autovalores dos operadores $\tilde{B}_{n}$ e $B_{n}$ são respectivamente $\lambda_{k}^{n^{3}}$ e $\lambda_{k}$, com 
$k=0, \cdots . n-1$. Neste caso nós temos que:

$$
\left|\lambda_{k}^{n^{3}}-\lambda_{k}\right|=(k \pi)^{2}\left|\left(\frac{\operatorname{sen} \frac{k \pi}{2 n^{3}}}{\frac{k \pi}{2 n^{3}}}\right)^{2}-1\right|
$$

Usando a expansāo em série de potência da função seno, obtemos:

$$
\left|\lambda_{k}^{n^{3}}-\lambda_{k}\right| \leq(k \pi)^{2}\left|\frac{2}{3 !}\left(\frac{k \pi}{2 n^{3}}\right)^{2}+o\left(\left(\frac{k \pi}{2 n^{3}}\right)^{3}\right)\right|
$$

O que nós dá para $k, k=0, \cdots, n-1$

$$
\left|\lambda_{k}^{n^{3}}-\lambda_{k}\right| \leq(n \pi)^{2}\left|\frac{2}{3 !}\left(\frac{\pi}{2 n^{2}}\right)^{2}+o\left(\left(\frac{1}{n^{2}}\right)^{3}\right)\right|=o\left(\frac{1}{n}\right)
$$

$\log \left|\lambda_{k}^{n^{3}}-\lambda_{k}\right| \rightarrow 0$ para todo $k=0, \cdots, n-1$ uniformemente, quando $n \rightarrow \infty$.

\subsubsection{Convergência Uniforme das Autofunções}

Nós mostraremos que $\left\|\nu_{k}^{n^{3}}(x)-\sqrt{2} \cos (k \pi x)\right\|_{\infty} \leq \epsilon$ para $n$ suficientemente grande e para todo $k=0 . \cdots, n-1$. Primeiramente, nós consideramos $\left|\cos (k \pi x)-\cos \left(k \pi x_{j}\right)\right|$ para $x \in\left[\frac{j-1}{n^{3}}, \frac{j}{n^{3}}\right]$.

Para $x \in\left[\frac{j-1}{n^{3}}, \frac{3}{n^{3}}\right]$ nós temos que

$$
\left|\cos (k \pi x)-\cos \left(k \pi x_{j}\right)\right| \leq k \pi \frac{1}{2 n^{3}}
$$

Para todo $k=0, \cdots, n-1$ e para todo $j=1, \cdots, n^{3}$ nós temos:

$$
\left|\cos (k \pi x)-\cos \left(k \pi x_{j}\right)\right| \leq \frac{\pi}{2 n^{2}}
$$

e assim, $\left\|\nu_{k}^{n^{3}}(x)-\sqrt{2} \cos (k \pi x)\right\|_{\infty} \leq \frac{c}{n^{2}}$, para alguma constante $c$.

\subsection{Comparação dos Campos Vetoriais}

Agora mostramos a proximidade dos campos vetoriais. Denotamos por $\bar{g}_{n}^{i}$ e $g_{n}^{i}$ a $i$-ésima função coordenada de $\overline{g_{n}}$ e $g_{n}$ respectivamente. Então. temos: 


$$
\begin{aligned}
& \left|g_{n}^{j}(v, 0)-\tilde{g}_{n}^{j}(v, 0)\right| \\
& \quad=\left|\sum_{k=1}^{n^{3}} \frac{1}{n^{3}} \nu_{(j-1) k}^{n^{3}} f\left(\sum_{l=1}^{n} \nu_{(l-1) k}^{n^{3}} v_{l}\right)-\int_{0}^{1} f\left(\sum_{l=1}^{n} v_{l} \sqrt{2} \cos (l-1) \pi x\right) \sqrt{2} \cos (j-1) \pi x d x\right| \\
& \quad \leq\left|\sum_{k=1}^{n^{3}} \int_{\frac{k-1}{n^{3}}}^{\frac{k}{n^{3}}}\left(\nu_{(j-1) k}^{n^{3}} f\left(\sum_{l=1}^{n} \nu_{(l-1) k}^{n^{3}} v_{l}\right)-f\left(\sum_{l=1}^{n} v_{l} \sqrt{2} \cos (l-1) \pi x\right) \sqrt{2} \cos (j-1) \pi x\right) d x\right| \\
& \quad \leq\left|\sum_{k=1}^{n^{3}} \int_{\frac{k-1}{n^{3}}}^{\frac{k}{n^{3}}} f\left(\sum_{l=1}^{n} \nu_{(l-1) k}^{n^{3}} v_{l}\right)\left(\nu_{(j-1) k}^{n^{3}}-\sqrt{2} \cos (j-1) \pi x\right) d x\right| \\
& \quad+\left|\sum_{k=1}^{\frac{k}{n^{3}}} \int_{\frac{k-1}{n^{3}}}\left(f\left(\sum_{l=1}^{n} \nu_{(l-1) k}^{n^{3}} v_{l}\right)-f\left(\sum_{l=1}^{n} v_{l} \sqrt{2} \cos (l-1) \pi x\right)\right) \sqrt{2} \cos ((j-1) \pi x) d x\right| . \\
& \quad \leq \sum_{k=1}^{\frac{k}{n^{3}}} \int_{\frac{k-1}{n^{3}}}^{\frac{k}{n^{3}}}\left|f\left(\sum_{l=1}^{n} \nu_{(l-1) k}^{n^{3}} v_{l}\right)\right|\left|\left(\nu_{(j-1) k}^{n^{3}}-\sqrt{2} \cos (j-1) \pi x\right)\right| d x \\
& \quad+\sum_{k=1}^{n^{3}} \int_{\frac{k-1}{n^{3}}}^{\frac{k}{n^{3}}}\left|f\left(\sum_{l=1}^{n} \nu_{(l-1) k}^{n^{3}} v_{l}\right)-f\left(\sum_{l=1}^{n} v_{l} \sqrt{2} \cos (l-1) \pi x\right) \| \sqrt{2} \cos ((j-1) \pi x)\right| d x \\
& \quad \leq|| f \|_{\infty} \frac{c}{n^{2}}+L_{f} \frac{c \sqrt{2}}{n^{2}} \sum_{l=1}^{n}\left|v_{l}\right| .
\end{aligned}
$$

Portanto,

$$
\begin{aligned}
\left\|g_{n}\left(v, \sigma_{n}(v)\right)-\tilde{g}_{n}\left(v, \tilde{\sigma}_{n}(v)\right)\right\|_{Y_{n}} & \leq\left\|g_{n}\left(v, \sigma_{n}(v)\right)-g_{n}(v, 0)\right\|_{Y_{n}}+\left\|g_{n}(v, 0)-\tilde{g}_{n}(v, 0)\right\|_{Y_{n}} \\
& +\left\|\tilde{g}_{n}(v, 0)-\tilde{g}_{n}\left(v, \tilde{\sigma}_{n}(v)\right)\right\|_{Y_{n}} \\
& \leq L_{f}\left\|\sigma_{n}(v)\right\|+L_{f}\left\|\tilde{\sigma}_{n}(v)\right\|+\frac{1}{\sqrt{n}}\left(\|f\|_{\infty} \frac{c}{n}+L_{f} \xi c \sqrt{2}\right):
\end{aligned}
$$

onde $\xi$ é como em (2.8).

Desde que, pelo teorema $1.9,\left\|\sigma_{n}\right\| \| \rightarrow 0$ e $\left\|\tilde{\sigma_{n}}\right\| \| \rightarrow 0$ quando $n \rightarrow \infty$ então

$$
\left\|g_{n}\left(v, \sigma_{n}(v)\right)-\tilde{g}_{n}\left(v, \tilde{\sigma}_{n}(v)\right)\right\| \rightarrow 0
$$

quando $n \rightarrow \infty$.

Similarmente, usando o fato que $f^{\prime}$ é globalmente Lipschitz, $\left\|D \sigma_{n}\right\| \rightarrow 0$ e $\left\|D \tilde{\sigma_{n}}\right\| \rightarrow 0$ quando $n \rightarrow \infty$, nós mostramos que as funções $g_{n}\left(v, \sigma_{n}(v)\right)$ e $\tilde{g}_{n}\left(v, \tilde{\sigma}_{n}(v)\right)$ são $C^{1}$ próximas. Assim, nós temos o seguinte teorema:

Teorema 2.3. Seja $f \in C^{2}(R, R)$ uma função limitada com primeira e segunda derivadas limitada. Assuma que o fluxo sobre $\mathcal{A}$ é estruturalmente estável. Então, para $n$ 
suficientemente grande o fluxo de (2.20) sobre o atrator $\mathcal{A}_{n}$ e o fluxo de (2.9) sobre $\tilde{\mathcal{A}}_{n}$ são topologicamente equivalentes.

Demonstraçāo: Nós primeiro observamos que, de [He2], nós temos que (0.1) é genericamente Morse-Smale e portanto, nossa suposição sobre a estabilidade estrutural não é uma restrição tão forte para a classe de aplicaçōes $f$ em consideração. Assim, nós temos que (0.1) é $\mathcal{A}$-estruturalmente estável.

Nós também temos que o campo de vetores de (2.20) é uma perturbação $C^{1}$ pequena do (2.9) e o teorema está provado. 


\section{Capítulo 3}

\section{Semi-continuidade superior e inferior} de atratores dos problemas da ondá

\section{com atrito forte e sem atrito forte}

\section{Introdução}

Nesta parte do trabalho vamos mostrar a semi-continuidade superior e inferior dos atratores para os problemas

$$
\begin{aligned}
& u_{t t}+2 \eta\left(-\Delta+\frac{\delta}{2}\right)^{1 / 2} u_{t}+2 a u_{t}=u_{x x}+f(u), \quad 0<x<1, t>0 \\
& u_{x}(0)=u_{x}(1)=0, t>0
\end{aligned}
$$

e

$$
\begin{aligned}
& u_{t t}+2 a u_{t}=u_{x x}+f(u), \quad 0<x<1, t>0 \\
& u_{x}(0)=u_{x}(1)=0, t>0,
\end{aligned}
$$

onde $\eta>0, a>0$.

A continuidade dos atratores é estudada em relação ao parâmetro $\eta$, em $\eta=0$.

Como no capítulo 1, trabalharemos com as equações anteriores nas seguintes formas:

$$
\begin{aligned}
\frac{d^{2} u}{d t^{2}}+2 \eta A^{\frac{1}{2}} \frac{d u}{d t}+2 a \frac{d u}{d t} & =-A u+f^{e}(u), \\
\frac{d^{2} u}{d t^{2}}+2 a \frac{d u}{d t} & =-A u+f^{e}(u),
\end{aligned}
$$


ou

$$
\frac{d}{d t}\left[\begin{array}{l}
u \\
v
\end{array}\right]=C_{\eta}\left[\begin{array}{l}
u \\
v
\end{array}\right]+h\left(\left[\begin{array}{l}
u \\
v
\end{array}\right]\right)
$$

onde $A=-\Delta+\frac{\delta}{2} I: D(A) \subset X \rightarrow X, D(A)=\left\{u \in H^{2} ; \frac{d u}{d x}(0)=\frac{d u}{d x}(1)=0\right\}, X=L^{2}=$ $X^{0}$

$$
C_{\eta}=\left[\begin{array}{cc}
0 & I \\
-A & -2\left(\eta A^{1 / 2}+a\right)
\end{array}\right] \text { e } h\left(\left[\begin{array}{l}
u \\
v
\end{array}\right]\right)=\left[\begin{array}{c}
0 \\
f^{e}(u)
\end{array}\right]
$$

e $D\left(C_{\eta}\right)=\mathrm{I}^{1} \times X^{\frac{1}{2}}, \eta \geq 0$

Pelos resultados do capítulo 1 , sabemos que geram $C^{1}$-semigrupos sobre $Y^{0}=H^{1} \times L^{2}$, são sistemas gradientes e assintoticamente suaves. Também temos a existência de um atrator global $\mathcal{A}_{\eta}$ para (3.1) e um atrator global $\mathcal{A}$ para (3.2). Sobre o espaço $Y^{0}$ temos o produto interno $((u, v),(w, z))_{Y^{0}}=\langle A u, w\rangle+\langle v, z\rangle$, sobre o espaço $Y^{1}$ temos o produto interno $((u, v),(w, z)\rangle_{Y^{1}}=(A u, A w\rangle+\langle A v, z\rangle$, onde $(.,$.$\rangle é o produto interno de L^{2}$.

A demonstração da semi-continuidade superior é análoga a dada em [HR1]. Primeiramente fazemos a limitação, uniforme em $\eta$, dos atratores $\mathcal{A}_{\eta}$ e $\mathcal{A}$ nas normas $H^{1} \times L^{2}$ e $H^{2} \times H^{1}$, sendo para isso necessário um funcional energia diferente do funcional clássico. A definição deste novo funcional enrolve uma constante que depende apenas do parâmetro $a$, como vernos no próximo lema. A demonstração da semi-continuidade inferior é feita utilizando um teorema de semi-continuidade inferior de [Ha2] e dois teoremas de semicontinuidade inferior para as variedades instáveis locais de $[\mathrm{CH}]$. estes teoremas são o Teorema 1.10 e os Teoremas 1.11 e 1.12, respectivamente e estão contidos no capítulo 1 .

\subsection{Semi-continuidade superior}

Como comentado acima, vamos utilizar um funcional energia alternativo e para sua definição é necessário uma nova constante, $b$. Esta constante será dada através do lema 3.1 . Com esta nova constante definimos o funcional energia e somos capazes de mostrar que os atratores $\mathcal{A}$ e $\mathcal{A}_{\eta}$ sāo uniformemente limitados nas normas $\mathrm{I}^{-0}$ e $Y^{1}$, mais que isso, os semigrupos gerados são limitado-dissipatiros, uniformemente em $\eta$ tanto no conjunto limitado quanto no tempo necessário para a atração. Além da limitaçāo dos atratores 
também mostramos a limitação, uniforme em $\eta$, da integral da norma $L^{2}$ da derivada em relação ao tempo. Com estas limitações utilizamos o Teorema de Arzelá-Ascoli e mostramos convergência de soluçōes sobre os atratores. Finalmente utilizamos o Lema 1.2 para obter a semi-continuidade superior dos atratores.

Esta seção contém três subseções. Na primeira subseção temos a limitação dos atratores na norma $Y^{0}$ e a limitação da derivada; na segunda subseção temos a limitação dos atratores na norma $Y^{1}$ e na terceira subseção temos a prova da semi-continuidade superior dos atratores.

Antes de passarmos à relação entre os atratores $\mathcal{A}_{\eta}$ e $\mathcal{A}$, vamos demonstrar algumas desigualdades que serão úteis nas subseções que se seguem.

Denotaremos por $\lambda_{1}$ o primeiro auto-valor do operador $A$; ou seja, $\lambda_{1}=\frac{\delta}{2}$.

Lema 3.1. Se b é um número real não negativo satisfazendo

$$
b \leq \inf \left\{\frac{1}{8}, \frac{\lambda_{1}}{4}, \frac{\sqrt{\lambda_{1}}}{4}, \frac{9 a \lambda_{1}}{4\left(3 \lambda_{1}+4 a^{2}\right)}\right\}
$$

então

$$
\begin{aligned}
& \frac{1}{2}\|\psi\|_{L^{2}}^{2}+2 b\langle\phi, \psi\rangle+\frac{1}{2}\|\phi\|_{H^{1}}^{2} \geq \frac{1}{4}\left(\|\psi\|_{L^{2}}^{2}+\|\phi\|_{H^{1}}^{2}\right) \\
& \frac{1}{2}\|\psi\|_{L^{2}}^{2}+2 b\langle\phi, \psi\rangle+\frac{1}{2}\|\phi\|_{H^{1}}^{2} \leq \frac{3}{4}\left(\|\psi\|_{L^{2}}^{2}+\|\phi\|_{H^{1}}^{2}\right)
\end{aligned}
$$

$e$

$$
(2 a-2 b)\|\psi\|_{L^{2}}^{2}+4 a b\langle\phi, \psi)+2 b\|\phi\|_{H^{1}}^{2} \geq \inf \left\{\frac{a}{2}, \frac{b}{2}\right\}\left(\|\psi\|_{L^{2}}^{2}+\|\phi\|_{H^{1}}^{2}\right)
$$

Demonstração: Para a demonstração de (3.7) e (3.8), basta observar que $\lambda_{1}\|u\|_{L^{2}}^{2} \leq$ $\|u\|_{H^{1}}^{2}$. Portanto, faremos somente a demonstração de (3.9). Pelas Desigualdades de Schwartz e de Young

$$
\begin{aligned}
(2 a-2 b)\|\psi\|_{L^{2}}^{2}+4 a b\langle\phi, \psi\rangle & +2 b\|\phi\|_{H^{1}}^{2} \geq(2 a-2 b)\|\psi\|_{L^{2}}^{2}-4 a b\|\phi\|_{L^{2}}\|\psi\|_{L^{2}}+2 b\|\phi\|_{H^{1}}^{2} \\
& \geq(2 a-2 b)\|\psi\|_{L^{2}}^{2}-4 a b\left(\frac{\epsilon\|\phi\|_{L^{2}}^{2}}{2}+\frac{\|\psi\|_{L^{2}}^{2}}{2 \epsilon}\right)+2 b\|\phi\|_{H^{1}}^{2} \\
& \geq(2 a-2 b)\|\psi\|_{L^{2}}^{2}-2 a b\left(\frac{\epsilon\|\phi\|_{H^{1}}^{2}}{\lambda_{1}}+\frac{\|\psi\|_{L^{2}}^{2}}{\epsilon}\right)+2 b\left\|_{\phi}\right\|_{H^{1}}^{2} \\
& \left.=\left((2 a-2 b)-\frac{2 a b}{\epsilon}\right)\|\psi\|_{L^{2}}^{2}\right)+\left(-\frac{2 a b \epsilon}{\lambda_{1}}+2 b\right)\|\phi\|_{H^{1}}^{2}
\end{aligned}
$$

Agora, fazemos a escolha de $\epsilon$ tal que (3.9) é satisfeito. Fazendo $\epsilon=\frac{3 \lambda_{1}}{4 a}$ obtemos que $\left(-\frac{2 a b \epsilon}{\lambda_{1}}+2 b\right)=\frac{b}{2}$. E assim, para $b \leq \frac{9 a \lambda_{1}}{4\left(3 \lambda_{1}+4 a^{2}\right)}$ temos $2\left(a-b-\frac{a b}{\epsilon}\right) \geq \frac{a}{2}$. 


\subsubsection{Limitação, uniforme em $\eta$, dos atratores na norma $Y^{0}$}

O teorema a seguir garante que existe um conjunto que absorve as órbitas de conjuntos limitados por $\left\{T_{\eta}(t), t \geq 0\right\}$, que este conjunto bem como o tempo de entrada são independentes de $\eta$.

Teorema 3.1. Seja $0<\eta<\eta_{0}$ então o sistema (3.1) é limitado dissipativo em $Y^{0}$, ou seja, existem constantes $C_{0}>0$ e para cada $r_{0}, \exists t_{0}=t\left(r_{0}\right)$ tal que

$$
\left\|T_{\eta}(t)(u, v)\right\|_{Y_{0}} \leq C_{0}, t \geq t_{0}
$$

para todo $(u, v)$ tal que $\|(u, v)\|_{Y_{0}} \leq r_{0}$

Demonstração: Fixe $b$ satisfazendo a hipótese do lema (3.1). Para qualquer $\eta>0$, defina o seguinte funcional energia sobre $Y_{0}$,

$$
V_{b}(\phi, \psi)=\frac{1}{2}\|\psi\|_{L^{2}}^{2}+\frac{1}{2}\|\phi\|_{H^{1}}^{2}+2 b\langle\psi, \phi)-\int_{0}^{1} F(\phi(x)) d x
$$

onde $F(v)=\int_{0}^{v} f(s) d s$

Seja $u_{\eta}\left(t, u_{0}, v_{0}\right)$ solução de (3.1), então

$$
\begin{aligned}
\frac{d}{d t} V_{b}\left(u_{\eta}(t), \frac{d u_{\eta}}{d t}(t)\right)=\frac{1}{2} & \frac{d}{d t}\left[\left\|\frac{d u_{\eta}}{d t}(t)\right\|_{L^{2}}^{2}+\left\|u_{\eta}(t)\right\|_{H^{1}}^{2}\right]+2 b\left\|\frac{d u_{\eta}}{d t}(t)\right\|_{L^{2}}^{2} \\
& +2 b\left\langle\frac{d^{2} u_{\eta}}{d t^{2}}(t), u_{\eta}(t)\right\rangle-\left\langle f\left(u_{\eta}(t)\right), \frac{d u_{\eta}(t)}{d t}(t)\right\rangle
\end{aligned}
$$

Desde que $\left\langle\frac{d^{2} u}{d t^{2}}(t)+A u, \frac{d u}{d t}\right\rangle=\frac{1}{2} \frac{d}{d t}\left(\left\|\frac{d u_{\eta}}{d t}(t)\right\|_{L^{2}}^{2}+\left\|u_{\eta}(t)\right\|_{H^{1}}^{2}\right)$, e, pela equação (3.1), obtemos

$$
\begin{aligned}
\frac{d}{d t} V_{b}\left(u_{\eta}(t), \frac{d u_{\eta}}{d t}(t)\right)= & \left\langle-2 \eta A^{\frac{1}{2}} \frac{d u_{\eta}}{d t}(t)-2 a \frac{d u_{\eta}}{d t}(t)+f\left(u_{\eta}(t)\right), \frac{d u_{\eta}}{d t}(t)\right\rangle+2 b\left\|\frac{d u_{\eta}}{d t}(t)\right\|_{L^{2}}^{2} \\
+2 b\left\langle f\left(u_{\eta}(t)\right)-\right. & \left.A u_{\eta}(t)-2 \eta A^{\frac{1}{2}} \frac{d u_{\eta}}{d t}(t)-2 a \frac{d u_{\eta}}{d t}(t), u_{\eta}(t)\right\rangle-\left\langle f\left(u_{\eta}(t)\right), \frac{d u_{\eta}}{d t}(t)\right\rangle \\
= & -2 \eta\left\|A^{\frac{1}{4}} \frac{d u_{\eta}}{d t}(t)\right\|_{L^{2}}^{2}+(2 b-2 a)\left\|\frac{d u_{\eta}}{d t}(t)\right\|_{L^{2}}^{2}-4 a b\left\langle\frac{d u_{\eta}}{d t}(t), u_{\eta}(t)\right\rangle \\
& -2 b\left\|u_{\eta}(t)\right\|_{H^{1}}^{2}+2 b\left\langle f\left(u_{\eta}(t)\right), u_{\eta}(t)\right\rangle-4 b \eta\left\langle A^{\frac{1}{2}} \frac{d u_{\eta}}{d t}(t), u_{\eta}(t)\right\rangle \\
\leq & -4 a b\left\langle\frac{d u_{\eta}}{d t}(t), u_{\eta}(t)\right\rangle+(2 b-2 a)\left\|\frac{d u_{\eta}}{d t}(t)\right\|_{L^{2}}^{2}+2 b\left\langle f\left(u_{\eta}(t)\right) . u_{\eta}(t)\right\rangle \\
-2 b\left\|u_{\eta}(t)\right\|_{H^{1}}^{2}+ & 4 b \eta\left\|A^{\frac{1}{4}} \frac{d u_{\eta}}{d t}(t)\right\|_{L^{2}}\left\|A^{\frac{1}{4}} u_{\eta}(t)\right\|_{L^{2}}-2 \eta\left\|\cdot A^{\frac{1}{4}} \frac{d u_{\eta}}{d t}(t)\right\|_{L^{2}}^{2}
\end{aligned}
$$

Como $b \leq \frac{1}{8}$, pela Desigualdade de Young e por interpolação. temos:

$$
4 b \eta\left\|A^{\frac{1}{4}} \frac{d u_{\eta}}{d t}(t)\right\| L_{L^{2}}\left\|A^{\frac{1}{4}} u_{\eta}(t)\right\|_{L^{2}}-2 \eta\left\|A^{\frac{1}{4}} \frac{d u_{\eta}}{d t}(t)\right\|_{L^{2}}^{2} \leq \eta K\left\|u_{\eta}(t)\right\|_{H^{1}}^{2}
$$


Então, por (3.11) e (3.9), temos de (3.10) que:

$$
\frac{d}{d t} V_{b}\left(u_{\eta}(t), \frac{d u_{\eta}}{d t}(t)\right) \leq-\frac{5}{8} k_{0}\left(\left\|\frac{d u_{\eta}}{d t}(t)\right\|_{L^{2}}^{2}+\left\|u_{\eta}(t)\right\|_{H^{1}}^{2}\right)+2 b\left\langle f\left(u_{\eta}(t)\right), u_{\eta}(t)\right\rangle
$$

para $\eta \leq \eta_{0}$ e $k_{0}=\inf \left\{\frac{a}{2}, \frac{b}{2}\right\}$.

Agora, por (1.3), temos que existe um $c_{\delta / 4}>0$ tal que

$$
\begin{aligned}
f(v) v & \leq-\frac{\delta}{4} v^{2}+c_{\delta / 4} \\
F(v) & \leq-\frac{\delta}{4} v^{2}+c_{\delta / 4}
\end{aligned}
$$

Assim, por (3.13), temos que:

$$
2 b\left\langle f\left(u_{\eta}(t)\right), u_{\eta}(t)\right\rangle \leq-\frac{\delta}{4}\left\|u_{\eta}(t)\right\|_{L^{2}}^{2}+c_{\delta / 4}
$$

Portanto, por (3.15), temos de (3.12) que

$$
\frac{d}{d t} V_{b}\left(u_{\eta}(t), \frac{d u_{\eta}}{d t}(t)\right) \leq-\frac{5}{8} k_{0}\left(\left\|\frac{d u_{\eta}}{d t}(t)\right\|_{L^{2}}^{2}+\left\|u_{\eta}(t)\right\|_{H^{1}}^{2}\right)+c_{\delta / 4}
$$

Verificamos também que o funcional $V_{b}$ é limitado inferiormente. De fato, por (3.14) e por (3.7), temos

$$
V_{b}\left(u_{\eta}(t), \frac{d u_{\eta}}{d t}(t)\right) \geq \frac{1}{4}\left(\left\|\frac{d u_{\eta}}{d t}(t)\right\|_{L^{2}}^{2}+\left\|u_{\eta}(t)\right\|_{H^{1}}^{2}\right)-c_{\delta / 4}
$$

Finalmente mostramos que dado $r_{0}>0$ existe $t_{0}=t\left(r_{0}\right)$ tal que para $\left(\left\|\frac{d u_{\eta}}{d t}(0)\right\|_{L^{2}}^{2}+\right.$ $\left.\left\|u_{\eta}(0)\right\|_{H^{1}}^{2}\right) \leq r_{0}$ então

$$
V_{b}\left(u_{\eta}(t), \frac{d u_{\eta}}{d t}(t)\right) \leq K_{0}
$$

para $t \geq t_{0}$.

De fato, seja $\bar{r}$ suficientemente grande tal que $-\frac{5}{8} k_{0} \bar{r}^{2}+c_{\delta / 4}=-1$. Então, se $\left(\left\|\frac{d u_{\eta}}{d t}(t)\right\|_{L^{2}}^{2}+\right.$ $\left.\left\|u_{\eta}(t)\right\|_{H^{1}}^{2}\right) \geq \bar{r}$, temos que $\frac{d}{d t} V_{b}\left(u_{\eta}(t), \frac{d u_{\eta}}{d t}(t)\right) \leq-1$.

Seja $M=\max \left\{V(u, v) ;\|u\|_{H^{1}}^{2}+\|v\|_{L^{2}}^{2} \leq \bar{r}\right\}$ e $C_{M}=\{(u, v): V(u . v\rangle \leq M\}$. Por (3.17), temos que $C_{M}$ é limitado. E, por definição, que $B_{\bar{r}}(0) \subset C_{M}$.

Seja $B_{r_{0}}$ : mostraremos que para cada $\left(u_{0}, v_{0}\right) \in B_{r_{0}}$ temos $T_{\eta}\left(t_{0}\right)\left(u_{0}, v_{0}\right) \in C_{M}$ para algum $t_{0}=t\left(u_{0}, v_{0}\right\rangle$ e que $\sup \left\{t\left(u_{0}, v_{0}\right) ;\left(u_{0}, v_{0}\right\rangle \in B_{r_{0}}\right\}<\infty$. De fato, temos dois casos a considerar.

i) Se $\left(u_{0}, v_{0}\right) \in C_{M}$ então $V\left(u_{0}, v_{0}\right) \leq M$. 
ii) Se $\left(u_{0}, v_{0}\right) \notin C_{M}$ então $\left(u_{0}, v_{0}\right) \notin B_{\tilde{r}}(0)$ então $\frac{d}{d t} V_{b}(u(t), v(t)) \leq-1$ para todo $t$ tal que $(u(t), v(t)) \notin B_{\bar{r}}(0), \operatorname{logo} V_{b}(u(t), v(t)) \leq V_{b}\left(u_{0}, v_{0}\right)-t$.

Assim, $V\left(u_{0}, v_{0}\right)>M$ então $V_{b}(u(t), v(t)) \leq K-t$, onde $K=\max \{V(u, v) ;(u, v) \in$ $\left.B_{r_{0}}\right\}$. Logo considere $t_{0}=K-M>0$. Observamos que $t_{0}$ foi escolhido uniforme em $B_{r_{0}}(0)$.

Verificamos que $C_{M}$ é positivamente invariante por $T_{\eta}(t)$. De fato, suponha por absurdo que existe um último tempo, $\bar{t}$, tal que $V\left(T_{\eta}(\bar{t})\left(u_{0}, v_{0}\right)\right)=M, T_{\eta}(t)\left(u_{0}, v_{0}\right) \in C_{M}$ para $t_{0} \leq t \leq \bar{t}$ e $T_{\eta}(t)\left(u_{0}, v_{0}\right) \notin C_{M}$ para $\bar{t}<t$, logo $T_{\eta}(t)\left(u_{0}, v_{0}\right) \notin B_{r_{0}}(0)$. Assim, $V\left(T_{\eta}(t)\left(u_{0}, v_{0}\right)\right)<V\left(T_{\eta}(\bar{t})\left(u_{0}, v_{0}\right)\right)=M$, absurdo.

Logo, para qualquer $B_{r_{0}}$ existe $t_{B}$ tal que $V\left(T_{\eta}\left(u_{0}, v_{0}\right)\right) \leq M$ para todo $t \geq t_{B}$ e conseqüentemente, por (3.17)

$$
\left(\left\|\frac{d u_{\eta}}{d t}(t)\right\|_{L^{2}}^{2}+\left\|u_{\eta}(t)\right\|_{H^{1}}^{2}\right) \leq C_{0}
$$

para $t \geq t_{B}$

Como corolário, temos

Corolário 3.1. Existe uma constante $C_{1}>0$ tal que

$$
\|\phi\|_{H^{1}}^{2}+\|\psi\|_{L^{2}}^{2} \leq C_{1}
$$

para toda $(\phi, v) \in \mathcal{A}_{\eta}$ e para todo $\eta, 0 \leq \eta \leq \eta_{0}$

Proposição 3.1. Para qualquer $0 \leq \eta \leq \eta_{0}$ e $r_{0}>0$ existe uma constante $C_{0}^{*}\left(r_{0}\right)$ tal que, para qualquer solução $u_{\eta}(t)$ de (3.1), com $\left(\left\|u_{0}\right\|_{H^{1}}^{2}+\left\|v_{0}\right\|_{L^{2}}^{2}\right)^{\frac{1}{2}} \leq r_{0}$, temos

$$
\int_{0}^{\infty}\left\|\frac{d u_{\eta}}{d t}(s)\right\|_{L^{2}}^{2} d s \leq C_{0}^{*}\left(r_{0}\right)
$$

Demonstração: Para qualquer $\eta>0$. Considere o funcional de Lyapunov

$$
V(\phi, \psi)=V_{0}(\phi, \psi)=\frac{1}{2}\|\psi\|_{L^{2}}^{2}+\frac{1}{2}\|\phi\|_{H^{1}}^{2}-\int_{0}^{l} F(\dot{\phi}(x)) d x,
$$

onde $F(v)=\int_{0}^{v} f(s) d s$.

Seja $u_{\eta}$ a solução de (3.1) com condição inicial $\left(u_{0} . v_{0}\right)$. então. de (3.10) com $b=0$. obtemos que

$$
\frac{d}{d t} V\left(u_{\eta}(t) \cdot \frac{d u_{\eta}}{d t}(t)\right) \leq-2 a\left\|\frac{d u_{\eta}}{d t}(t)\right\|_{L^{2}}^{2}-2 \eta\left\|A^{\frac{1}{4}} \frac{d u_{\eta}}{d t}(t)\right\|_{L^{2}}^{2} \leq-2 a\left\|\frac{d u_{\eta}}{d t}(t)\right\|_{L^{2}}^{2} .
$$


Logo,

$$
V\left(u_{\eta}(t), \frac{d u_{\eta}}{d t}(t)\right)-V\left(u_{0}, v_{0}\right) \leq-2 a \int_{0}^{t}\left\|\frac{d u_{\eta}}{d t}(t)\right\|_{L^{2}}^{2} d s
$$

ou seja,

$$
\int_{0}^{t}\left\|\frac{d u_{\eta}}{d t}(t)\right\|_{L^{2}}^{2} d s \leq \frac{1}{2 a}\left(V\left(u_{0}, v_{0}\right)-V\left(u_{\eta}(t), \frac{d u_{\eta}}{d t}(t)\right)\right)
$$

Como,

i) $V(\phi, \psi) \geq c\left(\|\phi\|_{H^{1}}^{2}+\|\psi\|_{L^{2}}^{2}\right)-c^{\prime}$

ii) $V(\phi, \psi) \leq c_{1}\left(r_{0}\right)\left(\|\phi\|_{H^{1}}^{2}+\|\psi\|_{L^{2}}^{2}\right)$;

Logo,

$$
\begin{array}{r}
\int_{0}^{t}\left\|\frac{d u_{\eta}}{d t}(t)\right\|_{L^{2}}^{2} d s \leq \frac{1}{2 a}\left(V\left(u_{0}, v_{0}\right)-V\left(u_{\eta}(t), \frac{d u_{\eta}}{d t}(t)\right)\right) \\
\leq \frac{1}{2 a}\left(V\left(u_{0}, v_{0}\right)-c\left(\left\|u_{\eta}(t)\right\|_{H^{1}}^{2}+\left\|\frac{d u_{\eta}}{d t}(t)\right\|_{L^{2}}^{2}\right)+c^{\prime}\right) \leq \frac{1}{2 a}\left(V\left(u_{0}, v_{0}\right)+c^{\prime}\right)
\end{array}
$$

E assim,

$$
\int_{0}^{\infty}\left\|\frac{d u_{\eta}}{d t}(t)\right\|_{L^{2}}^{2} d s=\lim _{t \rightarrow \infty} \int_{0}^{t}\left\|\frac{d u_{\eta}}{d t}(t)\right\|_{L^{2}}^{2} d s \leq \frac{1}{2 a}\left(V\left(u_{0}, v_{0}\right)+c^{\prime}\right)
$$

Por ii), temos

$$
\int_{0}^{\infty}\left\|\frac{d u_{\eta}}{d t}(t)\right\|_{L^{2}}^{2} d s \leq \frac{1}{2 a}\left(V\left(u_{0}, v_{0}\right)+c^{\prime}\right) \leq c_{1}\left(r_{0}\right)\left(\left\|u_{0}\right\|_{H^{1}}^{2}+\left\|v_{0}\right\|_{L^{2}}^{2}\right)+c^{\prime}=C_{0}^{*}\left(r_{0}\right)
$$

Além disso, por (i) temos

$$
\begin{array}{r}
\left(\left\|\frac{d u_{\eta}}{d t}(t)\right\|_{L^{2}}^{2}+\left\|u_{\eta}(t)\right\|_{H^{1}}^{2}\right) \leq \frac{1}{c}\left(V\left(u_{\eta}(t), \frac{d u_{\eta}}{d t}(t)\right)+c^{\prime}\right) \\
\leq \frac{1}{c}\left(V\left(u_{0}, v_{0}\right)+c^{\prime}\right) \leq \frac{1}{c}\left(c_{1}\left(r_{0}\right)\left(\left\|v_{0}\right\|_{L^{2}}^{2}+\left\|u_{0}\right\|_{H^{1}}^{2}\right)+c^{\prime}\right)
\end{array}
$$

E assim, $\left(\left\|\frac{d u_{\eta}}{d t}(t)\right\|_{L^{2}}^{2}+\left\|u_{\eta}(t)\right\|_{H^{1}}^{2}\right) \leq C_{1}^{*}\left(r_{0}\right)$.

3.1.2 Limitação, uniforme em $\eta$, dos atratores na norma de $Y^{1}$

Nesta seção procuramos estimativas que permitem obter órbitas em $\mathcal{A}$ como limite de órbitas em $\mathcal{A}_{\eta}$. Estas estimativas devem entāo fornecer condiçōes de compacidade que permitem esta passagem ao limite. 
Teorema 3.2. Seja $0<\eta<\eta_{0}$ então existe uma constante $C_{2}>0$ e para cada $r_{0} e$ $r_{1}$, constantes $C_{2}^{*}\left(r_{0}\right)>0$ e $C_{3}^{*}\left(r_{1}\right)>0$, tal que para toda solução $u_{\eta}(t)$ de (3.1), com $\left(\left\|v_{0}\right\|_{L^{2}}^{2}+\left\|u_{0}\right\|_{H^{3}}^{2}\right) \leq r_{0} e\left(\left\|v_{0}\right\|_{H^{3}}^{2}+\left\|u_{0}\right\|_{H^{2}}^{2}\right) \leq r_{1}, i=0,1$,

$$
\left\|\frac{d^{2}}{d t^{2}} u_{\eta}(t)\right\|_{0}^{2}+\left\|\frac{d u_{\eta}}{d t}(t)\right\|_{H^{1}}^{2}+\left\|u_{\eta}(t)\right\|_{H^{2}}^{2} \leq C_{2}^{*}\left(r_{0}\right)+C_{3}^{*}\left(r_{1}\right) e^{-C_{2} t}
$$

para todo $t \leq 0$

\section{Demonstração:}

(1) Desde que $f \in C^{2}(\mathbb{R}, \mathbb{R})$ então $f^{e}: H^{1}(0,1) \rightarrow L^{2}(0,1)$ é uma aplicação $C^{1}$. Além disso, se $v \in H^{2}(0,1) \cap H^{1}(0,1)$ então $f^{\prime}(v)$ é uma aplicação linear de $L^{2}$ em $L^{2}$ e $\left\|f^{\prime}(v) w\right\|_{L^{2}}^{2} \leq\left\|f^{\prime}(v)\right\|_{\infty}^{2}\|w\|_{L^{2}}^{2}$. Desde que, para $v \in H^{1}$

$$
\|v\|_{\infty} \leq \sqrt{2}\left(\left\|v^{\prime}\right\|_{L^{2}}^{2}+\|v\|_{L^{2}}^{2}\right) \leq c\left\|v^{\prime}\right\|_{H^{1}}
$$

então $\left\|f^{\prime}(v)\right\|_{\infty}^{2} \leq \sqrt{2}\left(\left\|\left(f^{\prime}(v)\right)^{\prime}\right\|_{L^{2}}^{2}+\left\|f^{\prime}(v)\right\|_{L^{2}}^{2}\right)$. Temos também que:

i) $\left\|\left(f^{\prime}(v)\right)^{\prime}\right\|_{L^{2}}^{2} \leq c^{*}\left(\|v\|_{H^{1}}\right)\left\|v^{\prime}\right\|_{L^{2}}^{2}$;

ii) $\left\|f^{\prime}(v)\right\|_{L^{2}}^{2} \leq c^{* *}\left(\|v\|_{H^{1}}\right)$.

E assim

$$
\left\|f^{\prime}(v)\right\|_{\mathcal{L}\left(L^{2}, L^{2}\right)} \leq C^{*}\left(\|v\|_{H^{1}}\right)\left(1+\left\|r^{\prime}\right\|_{H^{1}}^{2}\right)
$$

2)Agora assumimos que $\left(\left\|v_{0}\right\|_{H^{1}}^{2}+\left\|u_{0}\right\|_{H^{2}}^{2}\right)^{\frac{1}{2}} \leq r_{1}$. Desde que $T_{\eta}(t)\left(u_{0}, v_{0}\right) \in C\left([0, \infty), Y^{-0}\right.$ $\cap C\left((0, \infty), Y^{-1}\right)$ então consideremos o seguinte problema:

$$
\begin{aligned}
& w_{t t}+2 \eta A^{1 / 2} w_{t}+2 a w_{t}+A w=f^{\prime}(u) \frac{d u_{\eta}}{d t}, \quad 0<x<1, t>0 \\
& w_{x}(0)=w_{x}(1)=0, t>0 \\
& w_{\eta}(0)=v_{0} . \frac{d w_{\eta}}{d t}(0)=-2 \eta A^{\frac{1}{2}}-2 a v_{0}-A u_{0}-f\left(u_{0}\right)
\end{aligned}
$$

Como $f^{\prime}\left(u_{\eta}\right) \frac{d u_{\eta}}{d t} \in C\left([0, \infty), L^{2}\right)$ e $\left(w_{\eta}(0), \frac{d w_{\eta}}{d t}(0)\right) \in Y^{0}$ então existe uma única solução $w_{\eta}(t, x)$ de (3.23). Como $\frac{d u_{\eta}}{d t}(t)$ satisfaz (3.23). então pela unicidade então $w_{\eta}(t, x)=$ $\frac{d u_{\eta}}{d t}(t)$.

Voltando ao funcional, fixemos uma constante $b$ satisfazendo a hipótese do lema (3.1) e definimos

$$
\Sigma_{b}(\phi, \psi)=\frac{1}{2}\|\psi\|_{L^{2}}^{2}+\frac{1}{2}\|\dot{\phi}\|_{H^{1}}^{2}+2 b(\imath \cdot 0)
$$


Desde que $\left(\frac{d^{2} u}{d t^{2}}(t)+A u, \frac{d u}{d t}\right)=\frac{1}{2} \frac{d}{d t}\left(\left\|\frac{d u_{\eta}}{d t}(t)\right\|_{L^{2}}^{2}+\left\|u_{\eta}(t)\right\|_{H^{1}}^{2}\right)$, e, por (3.23). obtemos

$$
\begin{array}{r}
\frac{d}{d t} V_{b}\left(w_{\eta}(t), \frac{d w_{\eta}}{d t}(t)\right) \\
=\left\langle-2 \eta A^{\frac{1}{2}} \frac{d w_{\eta}}{d t}(t)-2 a \frac{d w_{\eta}}{d t}(t)+f^{\prime}\left(u_{\eta}(t)\right) w_{\eta}, \frac{d w_{\eta}}{d t}(t)\right\rangle+2 b\left\|\frac{d w_{\eta}}{d t}(t)\right\|_{L^{2}}^{2} \\
+2 b\left\langle f^{\prime}\left(u_{\eta}(t)\right) w_{\eta}(t)-A w_{\eta}(t)-2 \eta A^{\frac{1}{2}} \frac{d w_{\eta}}{d t}(t)-2 a \frac{d w_{\eta}}{d t}(t), w_{\eta}(t)\right) \\
=-2 \eta\left\|A^{\frac{1}{4}} \frac{d w_{\eta}}{d t}(t)\right\|_{L^{2}}^{2}+(2 b-2 a)\left\|\frac{d w_{\eta}}{d t}(t)\right\|_{L^{2}}^{2}-4 a b\left\langle\frac{d w_{\eta}}{d t}(t), w_{\eta}(t)\right\rangle \\
-2 b\left\|w_{\eta}(t)\right\|_{H^{1}}^{2}+2 b\left\langle f^{\prime}\left(u_{\eta}(t) w_{\eta}(t)\right), w_{\eta}(t)\right) \\
-4 b \eta\left\langle A^{\frac{1}{2}} \frac{d w_{\eta}}{d t}(t), w_{\eta}(t)\right)+\left\langle f^{\prime}\left(u_{\eta}(t) w_{\eta}(t)\right), \frac{d w_{\eta}}{d t}(t)\right)
\end{array}
$$

Por (3.9), Desigualdade de Young e interpolação, temos de (3.24), para $\eta \leq \eta_{0}$, que

$$
\begin{array}{r}
\frac{d}{d t} V_{b}\left(w_{\eta}(t), \frac{d w_{\eta}}{d t}(t)\right) \leq-\frac{5 k_{0}}{8}\left(\left\|\frac{d w_{\eta}}{d t}(t)\right\|_{L^{2}}^{2}+\left\|w_{\eta}(t)\right\|_{H^{1}}^{2}\right) \\
+\left\|f^{\prime}\left(u_{\eta}\right) w_{\eta}\right\|_{L^{2}}\left\|\frac{d w_{\eta}}{d t}\right\|_{L^{2}}+\frac{2 b}{\sqrt{\lambda_{1}}}\left\|f^{\prime}\left(u_{\eta}\right) w_{\eta}\right\|_{L^{2}}\left\|w_{\eta}\right\|_{H^{1}} \\
\leq-\frac{5 k_{0}}{8}\left(\left\|\frac{d w_{\eta}}{d t}(t)\right\|_{L^{2}}^{2}+\left\|w_{\eta}(t)\right\|_{H^{1}}^{2}\right)+\frac{\left\|f^{\prime}\left(u_{\eta}\right) w_{\eta}\right\|_{L^{2}}^{2}}{2 k_{0}} \\
+\frac{k_{0}}{2}\left\|\frac{d w_{\eta}}{d t}\right\|_{L^{2}}^{2}+\frac{2 b^{2}}{k_{0} \sqrt{\lambda_{1}}}\left\|f^{\prime}\left(u_{\eta}\right) w_{\eta}\right\|_{0}^{2}+\frac{k_{0}}{2}\left\|w_{\eta}\right\|_{H^{1}}^{2} \\
\leq-\frac{k_{0}}{8}\left(\left\|\frac{d w_{\eta}}{d t}(t)\right\|_{L^{2}}^{2}+\left\|w_{\eta}(t)\right\|_{H^{1}}^{2}\right)+\left(\frac{1}{2 k_{0}}+\frac{2 b^{2}}{k_{0} \lambda_{1}}\right)\left\|f^{\prime}\left(u_{\eta}(t)\right) w_{\eta}(t)\right\|_{L^{2}}^{2}
\end{array}
$$

Pela equação (3.1), temos que

$$
A u_{\eta}=f\left(u_{\eta}\right)-\frac{d^{2} u_{\eta}}{d t^{2}}-2 \eta A^{\frac{1}{2}} \frac{d u_{\eta}}{d t}-2 a \frac{d u_{\eta}}{d t}
$$

Logo,

$$
\begin{aligned}
\left\|u_{\eta}\right\|_{H^{2}}= & \left\|A u_{\eta}\right\|_{L^{2}}=\left\|f\left(u_{\eta}\right)-\frac{d^{2} u_{\eta}}{d t^{2}}-2 \eta A^{\frac{1}{2}} \frac{d u_{\eta}}{d t}-2 a \frac{d u_{\eta}}{d t}\right\|_{L^{2}} \\
& \leq\left\|f\left(u_{\eta}\right)\right\|_{L^{2}}+\left\|\frac{d^{2} u_{\eta}}{d t^{2}}\right\|_{L^{2}}+\left\|2 \eta \frac{d u_{\eta}}{d t}\right\|_{H^{1}}+\left\|2 a \frac{d u_{\eta}}{d t}\right\|
\end{aligned}
$$


Assim;

$$
\begin{array}{r}
\left\|u_{\eta}\right\|_{H^{2}}^{2} \leq\left\|f\left(u_{\eta}\right)\right\|_{L^{2}}^{2}+2\left\|f\left(u_{\eta}\right)\right\|\left\|_{L^{2}}\right\| \frac{d^{2} u_{\eta^{\prime}}}{d t^{2}}\left\|_{L^{2}}+\right\| \frac{d^{2} u_{\eta}}{d t^{2}} \|_{L^{2}}^{2} \\
+2\left(\left\|2 \eta \frac{d u_{\eta}}{d t}\right\|_{H^{1}}+\left\|2 a \frac{u_{\eta}}{d t}\right\|\right)\left(\left\|f\left(u_{\eta}\right)\right\|_{L^{2}}+\left\|\frac{d^{2} u_{\eta}}{d t^{2}}\right\|_{L^{2}}\right) \\
+\left\|2 \eta \frac{d u_{\eta}}{d t}\right\|_{H^{1}}^{2}+2\left\|2 \eta \frac{d u_{\eta}}{d t}\right\|_{H^{1}}\left\|2 a \frac{u_{\eta}}{d t}\right\|_{L^{2}}+\left\|2 a \frac{u_{\eta}}{d t}\right\|_{L^{2}}^{2} \\
\leq(2+2 \eta+2 a)\left\|f\left(u_{\eta}\right)\right\|_{L^{2}}^{2}+(2+2 \eta+2 a)\left\|\frac{d^{2} u_{\eta}}{d t^{2}}\right\|_{L^{2}}^{2} \\
+\left(4 \eta+4 \eta^{2}+4 a \eta\right)\left\|\frac{d u_{\eta}}{d t}\right\|_{H^{1}}^{2}+\left(4 a+4 a \eta+4 a^{2}\right)\left\|\frac{d u_{\eta}}{d t}\right\|_{L^{2}}^{2} \\
\leq d\left(\left\|f\left(u_{\eta}\right)\right\|_{L^{2}}^{2}+\left\|\frac{d w_{\eta}}{d t}\right\|_{L^{2}}^{2}+\left\|w_{\eta}\right\|_{L^{2}}^{2}\right)+4 \eta(1+a+\eta)\left\|u_{\eta}\right\|_{H^{1}}^{2} \\
\leq c\left(\left\|f\left(u_{\eta}\right)\right\|_{L^{2}}^{2}+\left\|\frac{d w_{\eta}}{d t}\right\|_{L^{2}}^{2}+\left\|w_{\eta}\right\|_{H^{1}}^{2}\right)
\end{array}
$$

Como. pela Proposição (3.1), temos que $\left(\left\|\frac{d u_{\eta}}{d t}\right\|_{L^{2}}^{2}+\left\|u_{\eta}\right\|_{H^{1}}^{2}\right) \leq C_{1}^{*}\left(r_{0}\right)$, para $\left(\left\|u_{0}\right\|_{H^{1}}^{2}+\right.$ $\left.\left\|v_{0}\right\|_{L^{2}}^{2}\right) \leq r_{0}$. então por $(3.22)$, temos

$$
\begin{aligned}
\left\|f^{\prime}\left(u_{\eta}(t)\right) w_{\eta}(t)\right\|_{L^{2}}^{2} \leq C^{*}\left(\left\|u_{\eta}\right\|_{H^{1}}\right) \|(1 & \left.+\left\|u_{\eta}\right\|_{H^{1}}^{2}\right)\left\|w_{\eta}(t)\right\|_{L^{2}}^{2} \\
& \leq C_{2}\left(r_{0}\right)\left\|w_{\eta}(t)\right\|_{L^{2}}^{2}
\end{aligned}
$$

Portanto, por (3.27), (3.8) e (3.7), obtemos de (3.25), que

$$
\begin{aligned}
\frac{d}{d t} V_{b}\left(w_{\eta}(t), \frac{d w_{\eta}}{d t}(t)\right) \leq & -\frac{k_{0}}{8}\left(\left\|\frac{d w_{\eta_{l}}}{d t}(t)\right\|_{L^{2}}^{2}+\left\|w_{\eta}(t)\right\|_{H^{1}}^{2}\right) \\
& +\left(\frac{1}{2 k_{0}}+\frac{2 b^{2}}{k_{0} \lambda_{1}}\right)\left\|w_{\eta}(t)\right\|_{L^{2}}^{2} C_{2}\left(r_{0}\right) \\
\leq-\frac{k_{0}}{6}\left(\frac{1}{2}\left\|\frac{d w_{\eta}}{d t}(t)\right\|_{L^{2}}^{2}+\right. & \left.2 b\left\langle\frac{d w_{\eta}}{d t}(t), w_{\eta}(t)\right)+\frac{1}{2}\left\|w_{\eta}(t)\right\|_{H^{1}}^{2}\right) \\
& +\left(\frac{1}{2 k_{0}}+\frac{2 b^{2}}{k_{0} \lambda_{1}}\right)\left\|w_{\eta}(t)\right\|_{L^{2}}^{2} C_{2}\left(r_{0}\right) \\
\leq & -\frac{k_{0}}{6} V\left(w_{\eta}, \frac{d w_{\eta}}{d t}\right)+C_{2}^{*}\left(r_{0}\right)\left\|w_{\eta}\right\|_{0}^{2}
\end{aligned}
$$

Resolvendo a desigualdade diferencial, e utilizando a proposição (3.1) obtemos

$$
\begin{array}{r}
\digamma^{\cdot}\left(u_{\eta}(t), \frac{d w_{\eta}}{d t}(t)\right) \leq e^{-k_{0} / 6 t} V\left(w_{\eta}(0), \frac{d w_{\eta}}{d t}(0)\right)+C_{2}^{*}\left(r_{0}\right) \int_{0}^{\infty}\left\|u_{\eta}(s)\right\|_{L^{2}}^{2} d s \\
\leq e^{-k_{0} / 6 t} \Upsilon^{\top}\left(w_{\eta}(0), \frac{d w_{\eta}}{d t}(0)\right)+C_{2}^{*}\left(r_{0}\right) C_{0}^{*}\left(r_{0}\right)
\end{array}
$$

Assim. por (3.7) e (3.8), temos de (3.29) que

$$
\left(\left\|u_{\eta}(t)\right\|_{H^{1}}^{2}+\left\|\frac{d w_{\eta}}{d t}(t)\right\|_{L^{2}}^{2}\right) \leq 3 e^{-k_{0} / 6 t}\left(\left\|w_{\eta}(0)\right\|_{H^{1}}^{2}+\left\|\frac{d u_{\eta}}{d t}(0)\right\|_{L^{2}}^{2}\right)+C_{3}^{*}\left(r_{0}\right)
$$


Como visto anteriormente em (3.26), obtemos de (3.30) e da equação (3.1), que

$$
\begin{aligned}
\left(\left\|w_{\eta}(t)\right\|_{H^{1}}^{2}\right. & \left.+\left\|\frac{d w_{\eta}}{d t}(t)\right\|_{L^{2}}^{2}+\left\|u_{\eta}(t)\right\|_{H^{2}}^{2}\right) \leq c\left\|f\left(u_{\eta}\right)\right\|_{L^{2}}^{2}+(1+c)\left(\left\|w_{\eta}(t)\right\|_{H^{1}}^{2}+\left\|\frac{d w_{\eta}}{d t}(t)\right\|_{L^{2}}^{2}\right) \\
& \leq C_{4}^{*}\left(r_{0}\right)+(1+c) C_{3}^{*}\left(r_{0}\right)+(1+c) e^{-\frac{k}{6} t}\left(\left\|w_{\eta}(0)\right\|_{H^{1}}^{2}+\left\|\frac{d w_{\eta}}{d t}(0)\right\|_{L^{2}}^{2}\right)
\end{aligned}
$$

Lembrando que $\left\|\frac{d w_{\eta}}{d t}(t)\right\|_{L^{2}} \leq 2 \eta\left\|u_{0}\right\|_{H^{1}}+2 a\left\|v_{0}\right\|_{L^{2}}+\left\|u_{0}\right\|_{H^{2}}+\left\|f\left(u_{0}\right)\right\|_{L^{2}}$ obtemos, de (3.31), que

$$
\left(\left\|w_{\eta}(t)\right\|_{H^{1}}^{2}+\left\|\frac{d w_{\eta}}{d t}(t)\right\|_{L^{2}}^{2}+\left\|u_{\eta}(t)\right\|_{H^{2}}^{2}\right) \leq C_{5}^{*}\left(r_{0}\right)+C_{6}^{*} e^{-\frac{k}{6} t} r_{1}
$$

Portanto, seguem os corolários:

Corolário 3.2. Seja $0<\eta \leq \eta_{0}$. O problema (3.1) é limitado dissipativo em $Y^{1}$, uniformemente em $\eta$, ou seja, existe um conjunto $B \subset Y^{1}, B$ limitado tal que para todo subconjunto $C \subset Y^{1}, C$ limitado existe $t_{1}=t\left(r_{0}, r_{1}\right)$ e tal que

$$
T_{\eta}(t)(C) \subset B, \text { para } t \leq t_{1}
$$

Corolário 3.3. Seja $0<\eta \leq \eta_{0}$. Então existem constantes $C_{3}$ e $C_{4}$ tais que: i)para todo $(\phi, \psi) \in \mathcal{A}_{\eta}$ temos

$$
\|\phi\|_{H^{2}}^{2}+\|\psi\|_{H^{1}}^{2} \leq C_{3}
$$

ii)para toda órbita $\left(u_{\eta}(t), \frac{d u_{\eta}}{d t}(t)\right) \in \mathcal{A}_{\eta}$ temos

$$
\left\|\frac{d^{2} u_{\eta}}{d t^{2}}(t)\right\|_{L^{2}}^{2} \leq C_{4}
$$

\subsubsection{Semicontinuidade superior dos atratores $\mathcal{A}_{\eta}$ em $\eta=0$}

Teorema 3.3. Os atratores globais, $\mathcal{A}_{\eta}$, dos problemas (3.1) são semicontínuos superiormente em $\eta=0, \mathcal{A}_{0}:=\mathcal{A}$; ou seja, $\forall \epsilon>0, \exists \eta_{0}$ tal que $\eta \leq \eta_{0}$ temos

$$
\delta_{Y^{0}}\left(\mathcal{A}_{\eta}, \mathcal{A}\right) \leq \epsilon
$$

Demonstração: Pelo Lema 1.2, para provar este teorema. é suficiente provar que: 
Afirmação 1. Seja $\eta_{k}$ uma sequência de números positivos tal que $\lim _{k \rightarrow \infty} \eta_{k}=0$ e seja $\left(u_{k}(t), \frac{d u_{k}}{d t}(t)\right)$ uma sequência de soluções de (3.1) com $\eta=\eta_{k}$ e tal que $\left(u_{k}(t) \cdot \frac{d u_{k}}{d t}(t)\right)$ $\in \mathcal{A}_{\eta_{k}}$ para todo $t \geq 0$. Então existe uma subsequência de $\eta_{k_{\jmath}}$ tal que $\left(u_{k_{\jmath}}(0)\right.$. $\left.\frac{d u_{k_{j}}}{d t}(0)\right)$ converge para $(\bar{u} \cdot \bar{v}) \in \mathcal{A}$ em $Y_{0}$.

Demonstração da afirmação (1). De fato: Sabemos, pelo corolário 3.3 que existe um conjunto $B$ limitado em $Y^{1}$ tal que

$$
\left(\bigcup_{\eta} \mathcal{A}_{\eta}\right) \cup \mathcal{A} \subset B
$$

Assim, $\cup_{t} \cup_{k} \cdot u_{k}(t)$ é um conjunto relativamente compacto de $H^{1}$ e a família de aplicações $u_{k}(t) \in C\left(\mathbb{R} . H^{1}\right)$, é equicontínua de $\mathbb{R}$ em $H^{1}$.

Seja $J_{m}, m \geq 0$, uma sequência de intervalos compactos de $\mathbb{R}$ tal que $J_{m} \subset J_{m+1}, m \geq 0$ e $\cup_{m} J_{m}=\mathbb{R}$. Pelo teorema de Arzelà-Ascoli, existe uma subsequência $u_{k_{0}}$ de $u_{k}$ tal que $u_{k_{0}}$ converge para $\bar{u}$ em $C\left(J_{0}, H^{1}\right)$. Por indução, aplicando o teorema de ArzelàAscoli à subsequência $u_{k_{m}}$ extraimos uma subsequência $u_{k_{m+1}}$ que converge para $\bar{u}$ em $C\left(J_{m+1}, H^{1}\right)$. Considerando a sequência diagonal, existe uma subsequência de números positivos $\eta_{j_{k}}$ de $\eta_{k}$ e a correspondente subsequência $u_{j_{k}}$ de $u_{k}$ que são soluções de (3.1) $\operatorname{com} \eta=\eta_{j k}$ e tal que $u_{j_{k}} \rightarrow \bar{u}$ em $C\left(J, H_{N}^{1}\right)$ para todo intervalo $J$ compacto. Temos que

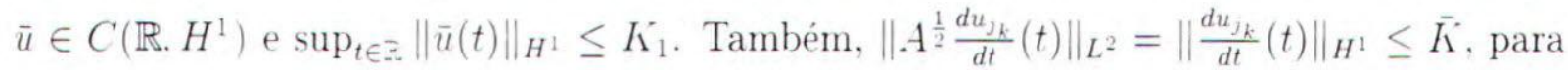
todo $t$ e para todo $\eta_{j k}$. Portanto.

$$
\sup _{t \in \mathbb{R}}\left(2 \eta_{j_{k}}\left\|A^{\frac{1}{2}} \frac{d u_{j_{k}}}{d t}(t)\right\|_{L^{2}}\right) \rightarrow 0
$$

quando $j_{k} \rightarrow x$.

Sabemos que. $\left\|\frac{d u_{j_{k}}}{d t}(t)\right\|_{H^{1}} \leq K$ e $\left\|\frac{d^{2} u_{j_{k}}}{d t^{2}}(t)\right\|_{L^{2}} \leq K$, para todo $t \in \mathbb{R}$ e para todo $j_{k}$ assim. $\frac{d u_{J_{k}}}{d t}(t) \in L^{\infty}\left(J, H^{1}\right)$ e $\frac{d^{2} u_{j_{k}}}{d t^{2}} d t \in L^{\infty}\left(J, L^{2}\right)$. Logo, $\frac{d u_{j_{k}}}{d t}(t) \in W^{1, \infty}\left(J . L^{2}\right)$ mas. $U^{-1, \infty}\left(J . L^{2}\right) \hookrightarrow C\left(J . L^{2}\right)$ e a família $\frac{d u_{j k}}{d t}(t) \in C^{\mu}\left(J . L^{2}\right)$ e é limitada em $k$ ou seja. $\left\|\frac{d u_{j_{k}}}{d t}\right\|_{C \mu\left(J . L^{2}\right)} \leq K$. para todo $k$. Assim. a família $\frac{d u_{j_{k}}}{d t}$ é equicontínua em $C\left(J, L^{2}\right)$ e portanto $\overline{\left(\frac{d u_{J_{k}}}{d t}\right)_{k}}$ é compacto em $C\left(J, L^{2}\right)$. Logo. existe uma subsequência de $\frac{d u_{J_{k}}}{d t}$. que também será denotada por $\frac{d u_{j_{k}}}{d t}$ e tal que existe $\bar{\imath} \in C\left(J . L^{2}\right)$ tal que $\frac{d u_{j}}{d t} \rightarrow \bar{\imath}$ em $C\left(J . L^{2}\right)$. para todo compacto $J$. Pelo fato que $\frac{d}{d t}$ é um operador fechado e $u_{j k} \rightarrow \bar{u}$. então $\frac{d \bar{u}}{d t}=\bar{v}$ e $\sup _{t \in Z}\left\{\|\bar{v}(t)\|_{L^{2}}\right\} \leq \hat{K}$.

Desde que $(\bar{u} \cdot \bar{c})$ é globalmente definida e globalmente limitada em $Y^{0}$. resta apenas 
mostrar que $(\bar{u}, \bar{v})$ é solução fraca de (3.2). De fato, como $\left(u_{k}, v_{k}\right)$ satisfaz a equação integral:

$$
\left(u_{k}, v_{k}\right)=e^{C t}\left(u_{0 k}, v_{0 k}\right)+\int_{0}^{t} e^{C(t-s)}\left[\left(0, f\left(u_{k}(s)\right)\right)+\left(0,-2 \eta A^{\frac{1}{2}} v_{k}(s)\right)\right] d s,
$$

Desde que $\left(u_{0 k}, v_{0 k}\right) \rightarrow(\bar{u}(0), \bar{v}(0))$ em $H^{1} \times L^{2}$ então

$$
\left\|e^{C t}\left(\left(u_{0 k}, v_{0 k}\right)-(\bar{u}(0), \bar{v}(0))\right)\right\|_{Y^{0}} \leq M e^{\omega t}\left\|\left(u_{0 k}, v_{0 k}\right)-(\bar{u}(0), \bar{v}(0))\right\|_{Y^{0}}
$$

então $\left\|e^{C t}\left(\left(u_{0 k}, v_{0 k}\right)-(\bar{u}(0), \bar{v}(0))\right)\right\|_{Y^{0}} \rightarrow 0$ quando $k \rightarrow \infty$ e uniformemente para $t$ em compactos. Também $\left(0, f\left(u_{k}(s)\right)\right) \rightarrow(0, f(\bar{u}(s)))$ em $C\left(\mathbb{R}, H^{1} \times L^{2}\right)$ e uniformemente para $s$ em compactos. E como observado anteriormente,

$$
\left(2 \eta_{j_{k}}\left\|A^{\frac{1}{2}} v_{j_{k}}(s)\right\|_{L^{2}}\right) \rightarrow 0
$$

quando $j_{k} \rightarrow \infty$, uniformemente para $s$ em compactos.

E assim, $\quad e^{C t}\left(u_{0 k}, v_{0 k}\right)+\int_{0}^{t} e^{C(t-s)}\left[\left(0, f\left(u_{k}(s)\right)\right)+\left(0,-2 \eta A^{\frac{1}{2}} v_{k}(s)\right)\right] d s$ converge para $e^{C t}(\bar{u}(0), \bar{v}(0))+\int_{0}^{t} e^{C(t-s)}\left[(0, f(\bar{u}(s)))+\left(0,-2 \eta A^{\frac{1}{2}} \bar{v}(s)\right)\right] d s$ quando $k \rightarrow \infty$.

Mas $e^{C t}\left(u_{0 k}, v_{0 k}\right)+\int_{0}^{t} e^{C(t-s)}\left[\left(0, f\left(u_{k}(s)\right)\right)+\left(0,-2 \eta A^{\frac{1}{2}} v_{k}(s)\right)\right] d s=\left(u_{k}, v_{k}\right) \rightarrow(\bar{u}, \bar{v})$. Portanto,

$$
(\bar{u}, \bar{v})=e^{C t}(\bar{u}(0), \bar{v}(0))+\int_{0}^{t} e^{C(t-s)}\left[(0, f(\bar{u}(s)))+\left(0,-2 \eta A^{\frac{1}{2}} \bar{v}(s)\right)\right] d s,
$$

e é solução fraca de (3.2). Isto conclui a demonstração.

\subsection{Semi-continuidade inferior dos atratores}

Para mostrarmos a semi-continuidade inferior dos atratores utilizaremos os teoremas 1.10. 1.11 e 1.12. Como pode ser observado, uma das hipóteses do teorema 1.10 para a semicontinuidade inferior dos atratores é a semi-continuidade inferior das variedades instáveis locais dos pontos de equilíbrio. Assim, primeiramente mostraremos a semi-continuidade inferior das variedades instáveis e para isso começamos verificando as hipóeses do teorema 1.12. Verificadas estas hipóteses, passamos para a verificação das hipóteses do teorema 1.11 e finalmente verificamos as hipóteses do teorema 1.10 o qual conclui a semicontinuidade inferior dos atratores. 
Primeiramente, mostraremos a semi-continuidade inferior das variedades instáveis locais nos pontos de equilíbrio.

Como já foi mostrado no capítulo 1 , temos que os pontos de equilíbrio para os problemas (3.1) e (3.2) são os mesmos para qualquer valor do parâmetro $\eta>0$. Estes pontos de equilíbrio são dados por: $\Phi=(\phi, 0)$ onde $\phi$ satisfaz

$$
\phi^{\prime \prime}+f(\phi)=0
$$

Consideremos os pontos de equilíbrio $\Phi_{j}$. Para cada $\eta \geq 0$ e $\Phi_{j}$ sejam $S_{j \eta}$ e $U_{j \eta}$ as variedades estáveis e instáveis respectivamente, para o problema (3.1), linearizado em $\Phi_{j}$. Sejam $P_{j \eta}$ e $Q_{j \eta}$ as projeções espectrais sobre $U_{j \eta}$ e $S_{j \eta}$ respectivamente. E seja $W_{j \eta}$ a variedade instável de $\Phi_{j}$ para o problema não linear. Existe uma vizinhança $N_{j \eta}$ de $\Phi_{j}$ e um homeomorfismo, $\sigma_{j \eta}: N_{j \eta} \bigcap U_{j \eta} \rightarrow N_{j \eta} \cap W_{j \eta}$.

Consideremos as equações linearizadas em $\Phi_{j}$; ou seja,

$$
\frac{d}{d t}\left[\begin{array}{l}
u \\
v
\end{array}\right]=\left[\begin{array}{cc}
0 & I \\
-A & -2\left(\eta A^{1 / 2}+a\right)
\end{array}\right]\left[\begin{array}{l}
u \\
v
\end{array}\right]+\left[\begin{array}{c}
0 \\
f^{\prime}\left(\phi_{j}\right) u
\end{array}\right]
$$

Conforme Teorema 1.12 devemos mostrar que se

$$
C_{\eta}^{\prime}\left(\Phi_{j}\right)=\left[\begin{array}{cc}
0 & I \\
-A & -2\left(\eta A^{1 / 2}+a\right)
\end{array}\right]+\left[\begin{array}{cc}
0 & 0 \\
f^{\prime}\left(o_{j}\right) & 0
\end{array}\right]
$$

entāo existem $\sigma_{-}<0$ e $\sigma_{+}>0$ tais que o espectro de $A_{\eta}^{\prime}\left(\Phi_{j}\right) . \sigma\left(A_{\eta}^{\prime}\left(\Phi_{j}\right)\right)$ satisfaz:

$$
\sigma\left(C_{\eta}^{\prime}\left(\Phi_{j}\right)\right) \bigcap\left[\sigma_{-}, \sigma_{+}\right]=\emptyset
$$

Vamos mostrar primeiro que os autovalores, $\mu_{\eta k}$ : de $C_{\eta}^{\prime}\left(\Phi_{j}\right)$ que são complexos tem parte real menor ou igual a $-a$, ou seja, $\operatorname{Re}\left(\mu_{\eta k}\right) \leq-a$. De fato, se $\lambda$ é autovalor de $C_{\eta}^{\prime}(\odot)$ com respectiva autofunção $(u, v) \in H^{2} \times H^{1}$ então $\lambda$ e $(u, v)$ devem satisfazer:

$$
\lambda^{2} u(x)+2 a \lambda u(x)+2 \eta \lambda A^{\frac{1}{2}}(x)+A u(x)+f^{\prime}(o(x)) u(x)=0 .
$$

Se $\lambda \in \mathbb{C}$ então multiplicando (3.35) por $\bar{u}$ e integrando de 0 a 1 e considerando $u$ normalizado em $L^{2}$, obtemos:

$$
\lambda^{2}+2 a \lambda+2 \eta \lambda\left\|A^{1 / 4} u\right\|_{L^{2}}^{2}+\|u\|_{H^{1}}^{2}+\left.\int_{0}^{1} f^{\prime}(\phi) ! u(x)\right|^{2} d x=0,
$$


separando a parte imaginária obtemos:

$$
2 \operatorname{Re}(\lambda) \operatorname{Im}(\lambda)+2 a \operatorname{Im}(\lambda)+2 \eta \operatorname{Im}(\lambda)\left\|A^{1 / 4} u\right\|_{L^{2}}^{2}=0
$$

Desde que assumimos que $\lambda \in \mathbb{C}$ então:

$$
\operatorname{Re}(\lambda)=-\eta\left\|A^{1 / 4} u\right\|_{L^{2}}^{2}-a \leq-a
$$

Portanto, para mostrarmos que existe uma separação no espectro para todo valor do parâmetro $\eta$, basta nos preocuparmos apenas com os autovalores reais de $C_{\eta}^{\prime}(\phi)$. Assim, voltamos a (3.36), e consideramos apenas a parte real.

$$
\operatorname{Re}(\lambda)^{2}-\operatorname{Im}(\lambda)^{2}+2 a \operatorname{Re}(\lambda)+2 \eta \operatorname{Re}(\lambda)\left\|A^{1 / 4} u\right\|_{L^{2}}^{2}+\|u\|_{H^{1}}^{2}+\int_{0}^{1} f^{\prime}(\phi)|u(x)|^{2} d x=0 .
$$

Desde que estamos considerando apenas $\lambda \in \mathbb{R}$ então $\operatorname{Im}(\lambda)=0$ e obtemos:

$$
\operatorname{Re}(\lambda)^{2}+2 a \operatorname{Re}(\lambda)+2 \eta \operatorname{Re}(\lambda)\left\|A^{1 / 4} u\right\|_{L^{2}}^{2}+\|u\|_{H^{1}}^{2}+\int_{0}^{1} f^{\prime}(\phi)|u(x)|^{2} d x=0 .
$$

Resolvendo como equação de $2^{\circ}$ em $\operatorname{Re}(\lambda)$ obtemos :

$$
\operatorname{Re}(\lambda)=-a-\eta\left\|A^{1 / 4} u\right\|_{L^{2}}^{2} \pm \sqrt{\left(a+\eta\left\|A^{1 / 4} u\right\|_{L^{2}}^{2}\right)^{2}-\left(\int_{0}^{1} f^{\prime}(\phi)|u(x)|^{2} d x+\|u\|_{H^{1}}^{2}\right)}
$$

e portanto, $|\lambda|=|\operatorname{Re}(\lambda)| \leq K$ para algum $K>0$ independente de $\eta>0$ para $\eta$ suficientemente pequeno.

Com estes fatos, basta mostramos que os autovalores de $C_{\eta}^{\prime}(\phi)$ convergem para os autovalores de $C^{\prime}(\phi)$ quando $\eta \rightarrow 0$. Para isto, faremos a seguinte separação de $C_{\eta}^{\prime}(\phi)$.

$$
C_{\eta}^{\prime}(\phi)=C^{\prime}(\phi)+B_{\eta}
$$

onde $B_{\eta}=\left[\begin{array}{cc}0 & 0 \\ 0 & -2 \eta A^{1 / 2}\end{array}\right]$. Observamos primeiramente que $B_{\eta}$ é $C^{\prime}(\phi)$-relativamente limitado. De fato,

$$
\left\|B_{\eta}\left(\left[\begin{array}{l}
u \\
v
\end{array}\right]\right)\right\|_{H^{1} \times L^{2}}=2 \eta\left\|A^{1 / 2} v\right\|_{L^{2}}=2 \eta\|v\|_{H^{1}} \leq 2 \eta\left\|C^{\prime}(\phi)\left(\left[\begin{array}{l}
u \\
v
\end{array}\right]\right)\right\|_{H^{1} \times L^{2}} .
$$

Como, para $\lambda \in \rho\left(C^{\prime}(\phi)\right)$ e $\lambda$ tal que $\left\|B_{\eta}\left(\lambda-C^{\prime}(\phi)\right)^{-1}\right\|<1$, temos $\lambda \in \rho\left(C_{\eta}^{\prime}(\phi)\right)$ pois $\left(\lambda I-C_{\eta}^{\prime}(\phi)\right)^{-1}=\left(I-B_{\eta}\left(\lambda-C^{\prime}(\phi)\right)^{-1}\right)^{-1}\left(\lambda-C^{\prime}(\phi)\right)^{-1}$. Entāo vamos mostrar que dado 
$\epsilon>0$, existe $\eta_{0}$ tal que para $\eta \leq \eta_{0}$ temos que $K_{\epsilon} \subset \rho\left(C_{\eta}^{\prime}(\phi)\right)$, onde $K_{\epsilon}=B_{K} \backslash \bigcup_{j} B_{\epsilon}\left(\mu_{j}\right)$ e $\mu_{j} \in \sigma\left(C^{\prime}(\phi)\right)$. De fato,

$$
\begin{array}{r}
\left\|B_{\eta}\left(\lambda-C^{\prime}(\phi)\right)^{-1}\right\| \leq 2 \eta\left\|C^{\prime}(\phi)\left(\lambda-C^{\prime}(\phi)\right)^{-1}\right\| \\
=2 \eta\left\|\lambda\left(\lambda-C^{\prime}(\phi)\right)^{-1}-I\right\| \leq 2 \eta\left(1+\left\|\lambda\left(\lambda-C^{\prime}\right)^{-1}\right\|\right) \leq 2 \eta\left(1+|\lambda|\left\|R\left(\lambda, C^{\prime}(\phi)\right)\right\|\right)
\end{array}
$$

Para $\lambda \in K_{\epsilon}$, temos $\| R\left(\lambda, C^{\prime}(\phi) \| \leq M\right.$ e $|\lambda| \leq K$, e portanto

$$
\left\|B_{\eta}\left(\lambda-C^{\prime}(\phi)\right)^{-1}\right\| \leq 2 \eta(1+K M)<1, \text { para, } \eta \leq \eta_{0}=\frac{1}{4(1+K M)}
$$

Logo, $\lambda \in \rho\left(C_{\eta}^{\prime}(\phi)\right)$ e assim $\sigma\left(C_{\eta}^{\prime}(\phi)\right) \cap B_{K} \subset K_{\epsilon}$ para $\eta \leq \eta_{0}$. Portanto a hipótese (K2), do teorema (1.12) está satisfeita.

Lema 3.2. Seja $\Phi=(\phi, 0)$ um ponto de equilibrio de (3.1), para todo $\eta \leq 0$. A constante de Lipschitz da aplicação $g^{e}: H^{1} \times L^{2} \rightarrow H^{1} \times L^{2}$ dada por $g^{e}\left(\left[\begin{array}{l}u \\ v\end{array}\right]\right)(x)=g\left(x,\left[\begin{array}{l}u \\ v\end{array}\right]\right)$, onde

$$
g(x,(r, s))=\left[\begin{array}{c}
0 \\
f(r)
\end{array}\right]-\left[\begin{array}{c}
0 \\
f(\phi(x))
\end{array}\right]-\left[\begin{array}{cc}
0 & 0 \\
f^{\prime}(\phi(x)) & 0
\end{array}\right]\left[\begin{array}{c}
r-\phi(x) \\
s
\end{array}\right] .
$$

Então, a constante de Lipschitz de $g^{e}$ na bola de raio $\frac{1}{n}$ em torno de $\Phi$ vai para zera quanda $n \rightarrow \infty$.

Demonstraçāo: Sejam $(u, v)$ e $(w, z)$ pontos de $H^{1} \times L^{2}$ em $B_{\frac{1}{n}}(\Phi)$, então, pelo Teorema do Valor Médio, temos:

$$
\begin{array}{r}
\left\|g^{e}(u, v)-g^{e}(w, z)\right\|_{H^{1} \times L^{2}}^{2}=\left\|f(u)-f(w)-f^{\prime}(o)(u-w)\right\|_{L^{2}}^{2} \\
=\int_{0}^{1}\left[f(u(x))-f(w(x))-f^{\prime}(\phi)(u(x)-u(x))\right]^{2} d x \\
=\int_{0}^{1}\left[f^{\prime}\left(u(x)-t_{x}(u(x)-w(x))\right)-\left(f^{\prime}(\phi(x))\right]^{2}(u(x)-w(x))^{2} d x\right. \\
\leq \int_{0}^{1}\left[f^{\prime}\left(u(x)-t_{x}(u(x)-w(x))\right)-f^{\prime}(\dot{\phi}(x))\right]^{2} d x\|u-w\|_{H^{1}}^{2} \\
\leq K\left(\|u-u\|_{H^{1}}^{2}\right)\|u-w\|_{H^{1}}^{2}
\end{array}
$$

onde $K\left(\|u-w\|_{H^{1}}^{2}\right)$ rai para zero quando $\|u-w\|_{H^{1}}$ rai para zero.

Portanto, a hipótese (K1) do teorema (1.12) está satisfeita.

Também temos que

$$
\left\|T_{C_{\eta}^{\prime}(\Phi)}(t)(u, v)\right\|_{Y} \leq K e^{-\gamma t}\|(u, v)\|_{Y} ; \text { para } t \geq 0 \mathrm{e}(u, v) \in S_{j \eta}
$$




$$
\left\|T_{C_{\eta}^{\prime}(\Phi)}(t)(u, v)\right\|_{Y} \leq K e^{\gamma t}\|(u, v)\|_{Y}, \text { para } t \leq 0 \text { e }(u, v) \in U_{j \eta}
$$

para algum $\gamma>0$. Logo, as hipóteses do teorcma (1.12) estão satisfeitas e nós temos as hipóteses (J4), (J5) e (J6) do teorema (1.11)

Vamos mostrar que as hipóteses (J1), (J2), e (J3) também estão satisfeitas.

Para a hipótese (J1), vamos fazer a seguinte separação do operador $C_{\eta}(u, v)+h(u, v)$.

$$
C_{\eta}\left[\begin{array}{l}
u \\
v
\end{array}\right]=C\left[\begin{array}{l}
u \\
v
\end{array}\right]+\left[\begin{array}{c}
0 \\
-2 \eta A^{\frac{1}{2}} v+f(u)
\end{array}\right]=A\left[\begin{array}{l}
u \\
v
\end{array}\right]+g\left(\left[\begin{array}{l}
u \\
v
\end{array}\right]\right)+h\left(\left[\begin{array}{l}
u \\
v
\end{array}\right]\right)
$$

onde $g\left(\left[\begin{array}{l}u \\ v\end{array}\right]\right)=\left[\begin{array}{c}0 \\ -2 \eta A^{\frac{1}{2}} v\end{array}\right]$ e $h\left(\left[\begin{array}{l}u \\ v\end{array}\right]\right)=\left[\begin{array}{c}0 \\ f(u)\end{array}\right]$. Pela fórmula da variação das constantes temos:

$$
T_{\eta}(t)\left[\begin{array}{l}
u \\
v
\end{array}\right]=e^{C t}\left[\begin{array}{l}
u \\
v
\end{array}\right]+\int_{0}^{t} e^{C(t-s)}\left(g\left(T_{\eta}(s)\left(\left[\begin{array}{l}
u \\
v
\end{array}\right]\right)\right)+h\left(T_{\eta}(s)\left[\begin{array}{l}
u \\
v
\end{array}\right]\right)\right) d s
$$

e

$$
T(t)\left[\begin{array}{l}
u \\
v
\end{array}\right]=e^{C t}\left[\begin{array}{l}
u \\
v
\end{array}\right]+\int_{0}^{t} e^{C(t-s)} h\left(T(s)\left(\left[\begin{array}{l}
u \\
v
\end{array}\right]\right)\right) d s .
$$

Assim, para $0 \leq t \leq \tau$, temos:

$$
\begin{array}{r}
\left\|T_{\eta}(t)\left(\left[\begin{array}{l}
u \\
v
\end{array}\right]\right)-T(t)\left(\left[\begin{array}{l}
u \\
v
\end{array}\right]\right)\right\|_{Y} \leq \int_{0}^{t}\left\|e^{C(t-s)}\left(h\left(T_{\eta}(s)\left(\left[\begin{array}{l}
u \\
v
\end{array}\right]\right)\right)-h\left(T(s)\left(\left[\begin{array}{l}
u \\
v
\end{array}\right]\right)\right)\right)\right\| d s \\
+\int_{0}^{t}\left\|e^{C(t-s)} g\left(T_{\eta}(s)\left(\left[\begin{array}{l}
u \\
v
\end{array}\right]\right)\right)\right\| d s
\end{array}
$$

Usaremos que $e^{C t}$ é um semigrupo de contrações. Também temos que $h: H^{1} \times L^{2} \rightarrow$ $H^{1} \times L^{2}$ Lipschitz em limitados. Para $(u, v) \in \mathcal{A}$ temos que $\|(u, v)\|_{Y^{1}} \leq r_{1}$ e assim, pelo teorema (3.2), temos que $\left\|T_{\eta}(s)\left(\left[\begin{array}{l}u \\ v\end{array}\right]\right)\right\|_{Y^{1}} \leq K$ para todo $s \geq 0$ e $(u, v) \in \mathcal{A}$ então, por (3.39), temos

$$
\left.\left\|T_{\eta}(t)\left(\left[\begin{array}{l}
u \\
v
\end{array}\right]\right)-T(t)\left(\left[\begin{array}{l}
u \\
v
\end{array}\right]\right)\right\|_{Y} \leq \int_{0}^{t} L \| T_{\eta}(s)\left(\left[\begin{array}{l}
u \\
v
\end{array}\right]\right)-T(s)\left(\left[\begin{array}{l}
u \\
v
\end{array}\right]\right)\right) \| d s+2 \eta M K \tau
$$

Por Gronwall, temos

$$
\left\|T_{\eta}(t)\left(\left[\begin{array}{l}
u \\
v
\end{array}\right]\right)-T(t)\left(\left[\begin{array}{l}
u \\
v
\end{array}\right]\right)\right\|_{Y} \leq 2 \eta M K \tau e^{M L t} \leq 2 \eta M K \tau e^{M L \tau}
$$


e assirn, (J1) está verificada.

Desde que já mostramos a convergência em $\eta$, dos autovalores dos problemas linearizados então se os pontos de equilíbrio do problema (3.2) são hiperbólicos então os pontos de equilíbrio dos problemas (3.1) para $\eta \leq \eta_{0}$ também são hiperbólicos. E assim, a hipótese (J2) também está verificada.

Resta apenas verificarmos a hipótese (J3) para que tenhamos a semi-continuidade inferior das variedades instáveis. Mas isto segue do fato que os autovalores convergem e as projeções sobre as variedades instáveis lineares são sobre espaços de dimensão finita.

Vamos agora mostrar que os atratores globais, $\mathcal{A}_{\eta}$, dos problemas (3.1) são semicontínuos inferiormente em $\eta=0, \mathcal{A}_{0}:=\mathcal{A}$.

Teorema 3.4. Os atratores globais, $\mathcal{A}_{\eta}$, dos problemas (3.1) são semicontínuos info mente em $\eta=0, \mathcal{A}_{0}:=\mathcal{A}$.

Demonstração: As hipóteses $(H 1)_{0}$ e $(H 2)_{0}$ já foram verificadas na seção (1.3.2) do capítulo 1. Quanto a hipótese $(H 3)_{0}$ temos por [Hel] que os pontos de equilíbrio do problema parabólico são genericamente hiperbólicos logo são um número finito e portanto os pontos de equilíbrio do problema (3.2) também são genericamente hiperbólicos (conı argumento de perturbação da não linearidade $f$ ). A hipótese $(H 4)_{\eta}$ já foi verificada na seção (1.3.2) do capítulo 1. Como observado para a hipótese (J2). desde que $(H 3)_{0}$ é assegurada e temos a convergência, em $\eta$, de autovalores do problema linearizado para $\eta \rightarrow 0$ então $(H \tilde{j})_{\eta}$ é verificada. A hipótese $(H 6)_{\eta}$ já foi verificadas anteriormente. Assim, resta apenas verificarmos a hipótese $(H 7)_{\eta}$. De fato, mostraremos que dados $\epsilon>0$ e $\tau>0$, existem um número real $\delta^{*}=\delta(\epsilon, \tau)>0$ e um $\eta_{0}>0$ tal que

$$
\left\|T_{\eta}(t)(u, v)_{\eta}-T_{0}(t)(u, v)\right\|_{Y} \leq \epsilon
$$

para $0 \leq t \leq \tau,(u, v) \in \mathcal{A},(u, v)_{\eta} \in \mathcal{A}_{\eta},\left\|(u, v)-(u, v)_{\eta}\right\|_{Y} \leq \delta^{*}$ e $\eta \leq \eta_{0}$.

Pela hipótese (J1) e pelo fato $\left\|T_{\eta}(t)\left((u, v)_{\eta}\right)-T_{\eta}(t)((u, v))\right\| \leq e^{L t}\left\|(u, v)-(u, v)_{\eta}\right\|_{Y} \cdot$ temos

$$
\begin{array}{r}
\left\|T_{\eta}(t)(u, v)_{\eta}-T(t)(u, v)\right\|_{Y} \\
\leq\left\|T_{\eta}(t)(u, v)_{\eta}-T_{\eta}(t)(u, v)\right\|_{Y}+\left\|T_{\eta}(t)(u, v)-T(t)(u \cdot v)\right\|_{Y} \\
\leq e^{L t}\left\|(u, v)_{\eta}-(u, v)\right\|_{Y}+2 \eta M K \tau e^{M / L \tau}
\end{array}
$$

Assim, considere $\delta^{*}$ tal que $e^{L \tau}\left\|(u, v)_{\eta}-(u, v)\right\|_{Y} \leq \frac{\epsilon}{2}$ e escolha $\eta_{0}$ tal que $2 \eta . M K \tau e^{M L \tau}$ $\leq \frac{\epsilon}{2}$ para $\eta \leq \eta_{0}$ 
Assim, temos as hipóteses do teorema 1.10 e portanto temos a sem-continuidade inferior dos atratores $\mathcal{A}_{\eta}$ em $\eta=0, \mathcal{A}_{0}:=\mathcal{A}$. 


\section{Capítulo 4}

\section{Semi-continuidade superior e inferior}

\section{em $\eta$ para cada $n$}

Neste capítulo vamos estudar a semi-continuidade superior e inferior para os problemas

$$
\ddot{U}+2 \eta A_{n}^{1 / 2} \dot{U}+2 a \dot{U}=-A_{n} U+\mathrm{f}(U)
$$

e

$$
\ddot{U}+2 a \dot{U}=-A_{n} U+\mathrm{f}(U)
$$

onde $A_{n}$ é a matriz $n \times n$ dada por $A_{n}=\Delta_{n}+\frac{\delta}{2} I_{n}$ e $\mathrm{f}(U)=\left(f\left(u_{1}\right), \cdots, f\left(u_{n}\right)\right)^{\top}$. A continuidade é estudada em relaçāo ao parâmetro $\eta$ para cada $n$ fixo.

Colocando na forma de um sistema de equaçōes de $1^{a}$ ordem. temos

$$
\frac{d}{d t}\left[\begin{array}{l}
U \\
V
\end{array}\right]=C_{\eta n}\left[\begin{array}{l}
U \\
V
\end{array}\right]+H\left(\left[\begin{array}{l}
U \\
V
\end{array}\right]\right)
$$

e

$$
\frac{d}{d t}\left[\begin{array}{l}
U \\
V
\end{array}\right]=C_{n}\left[\begin{array}{l}
U \\
V
\end{array}\right]+H\left(\left[\begin{array}{l}
U \\
V
\end{array}\right]\right)
$$

onde $C_{\eta n}=\left[\begin{array}{cc}0 & I_{n} \\ -A_{n} & -2\left(\eta-A_{n}^{1 / 2}+a\right)\end{array}\right], C_{n}=\left[\begin{array}{cc}0 & I_{n} \\ -A_{n} & -2 a\end{array}\right]$ e $H\left(\left[\begin{array}{l}L^{\cdot} \\ \Gamma^{\cdot}\end{array}\right]\right)=\left[\begin{array}{c}0 \\ \mathrm{f}(U)\end{array}\right]$

Pelos resultados do capítulo 1, sabemos que geram $C^{2}$-semigrupos sobre $\mathbb{R}^{n} \times \mathbb{R}^{n}$, são sistemas gradientes e assintoticamente suaves. Também temos a existência de um atrator 
global $\mathcal{A}_{\eta, n}$ para (4.3) e um atrator global $\mathcal{A}_{n}$ para (4.4). Sobre o espaço $\mathbb{R}^{n} \times \mathbb{R}^{n}$ definimos os produtos internos $\langle(U, V),(W, Z))_{Y_{n}^{0}}=\left\langle A_{n} U, W\right\rangle+\langle V, Z\rangle$ e $\langle(U, V),(W ; Z)\rangle_{Y_{n}^{\prime 2}}=$ $\left\langle A_{n} U, A_{n} W\right)+\left\langle A_{n} T_{i} Z\right\rangle$. Denotamos por $Y_{n}^{0}$ o espaço $\mathbb{R}^{n} \times \mathbb{R}^{n}$ com o produto interno $\langle., .\rangle_{Y_{n}^{0}}$, e por $I_{n}^{1}$ o espaço $\mathbb{R}^{n} \times \mathbb{R}^{n}$ com o produto interno $\langle., .\rangle_{Y_{n}^{1}}$, onde $\langle.,$.$) é o produto$ interno de $L_{d}^{2}$.

As demonstrações das semi-continuidades superior e inferior relativamente ao parâmetro $\eta$ são similares às dadas para o caso contínuo. Neste caso, nossa principal preocupação é a uniformidade em relação ao parâmetro $n$, pois nosso objetivo inicial era obter a continuidade em $\eta$ uniforme em $n$. Apesar de todas as hipóteses necessárias nos teoremas utilizados serem uniformes em relação a $n$, na demonstração do teorema sobre semi-continuidade inferior ainda há uma quantidade que não pudemos obter uniforme em $n$. Apesar disso, muitas das estimativas apresentadas aqui são independentes de $n$ e conjecturamos que a semi-continuidade inferior possa ser obtida independente de $n$.

\subsection{Semi-continuidade superior}

A demonstração da semi-continuidade superior é análoga a dada para o caso contínuo. As estimativas de limitação são obtidas uniforme nos parâmetros $\eta$ e $n$. Observamos que estas estimatiras serão necessárias para a semi-continuidade superior estudada entre os problemas discreto e contínuo para $\eta$ fixo.

Esta seção contém três subseções. Na primeira subseção temos a limitação dos atratores na norma $Y_{n}^{0}$ e a limitação da derivada; na segunda subseção temos a limitação dos atratores na norma $I_{n}^{-1}$ e na terceira subseção temos a prova da semi-continuidade superior dos atratores.

Seja $\lambda_{1}$ o primeiro auto-valor do operador $A_{n}$, ou seja, $\lambda_{1}=\frac{\delta}{2}$. Observamos que $\lambda_{1}$ é o mesmo do capítulo 3 . Como no capítulo 3 temos um lema análogo para o caso discreto.

Lema 4.1. Se b é um número real não negativo satisfazendo

$$
b \leq \inf \left\{\frac{1}{8}, \frac{\lambda_{1}}{4}, \frac{\sqrt{\lambda_{1}}}{4}, \frac{9 a}{4} \frac{\lambda_{1}}{\left(3 \lambda_{1}+4 a^{2}\right)}\right\}
$$


então

$$
\begin{aligned}
& \frac{1}{2}\|V\|_{L_{d}^{2}}^{2}+2 b(U, V)+\frac{1}{2}\|U\|_{H_{d}^{1}}^{2} \geq \frac{1}{4}\left(\|U\|_{L_{d}^{2}}^{2}+\|\phi\|_{H_{d}^{1}}^{2}\right) \\
& \frac{1}{2}\|V\|_{L_{d}^{2}}^{2}+2 b\langle U, V)+\frac{1}{2}\|U\|_{H_{d}^{1}}^{2} \leq \frac{3}{4}\left(\|V\|_{L_{d}^{2}}^{2}+\left\|U^{\top}\right\|_{H_{d}^{1}}^{2}\right)
\end{aligned}
$$

$e$

$$
(2 a-2 b)\|V\|_{L_{d}^{2}}^{2}+4 a b\langle U, V)+2 b\|U\|_{H_{d}^{1}}^{2} \geq \inf \left\{\frac{a}{2}, \frac{b}{2}\right\}\left(\|V\|_{L_{d}^{2}}^{2}+\|U\|_{H_{d}^{2}}^{2}\right)
$$

\subsubsection{Limitação uniforme em $n$ e em $\eta$ dos atratores $\mathcal{A}_{\eta n}$ na norma de $Y_{n}^{0}$}

Mostraremos que os semigrupos $T_{\eta n}(t)$ são limitados dissipatiros e tanto o conjunto $B$ que atrai limitados sob $T_{\eta n}(t)$ quanto o tempo $\tau(C)$ a partir do qual $T_{\eta n}(t) C \subset B, C$ limitado, podem ser escolhidos independentes dos parâmetros $n$ e $\eta$ para $n$ suficientemente grande e $\eta$ suficientemente pequeno.

Teorema 4.1. Seja $0<\eta<\eta_{0}$ e $n \geq n_{0}$ então o sistema (4.3) é limitado dissipativo em $\mathbb{R}^{n} \times \mathbb{R}^{n}$, ou seja, existem constantes $C_{0}>0$ e para cada $r_{0}, \exists t_{0}=t\left(r_{0}\right)$ tal que

$$
\left\|T_{\eta n}(t)(U, V)\right\|_{Y_{n}^{0}} \leq C_{0}, t \geq t_{0}
$$

para todo $\left(U, \Gamma^{r}\right)$ tal que $\|(U, V)\|_{Y_{n}^{0}} \leq r_{0}$

Demonstração: Fixe $b$ satisfazendo a hipótese do Lema 4.1. Para qualquer $\eta>0 \mathrm{e}$ $n \geq n_{0}$, defina o seguinte funcional energia sobre $\mathbb{R}^{n} \times \mathbb{R}^{n}$,

$$
V_{n}(U, V)=\frac{1}{2}\left\langle A_{n} U, U\right\rangle+2 b\langle U, V)+\frac{1}{2}\langle V, V\rangle-\langle\bar{F}(U) . \overline{1})
$$

onde $\overline{1}=(1,1, \cdots, 1), \bar{F}=\left(\tilde{F}\left(u_{1}\right), \tilde{F}\left(u_{2}\right), \cdots, \tilde{F}\left(u_{n}\right)\right)$ e $\tilde{F}(u)=\int_{0}^{u} f(s) d s$. Desde que para $\left(\zeta_{0}, V_{0}\right) \in \mathbb{R}^{n} \times \mathbb{R}^{n}$ temos $\left[\begin{array}{l}U\left(t, U_{0}, V_{0}\right) \\ V\left(t, U_{0}, V_{0}\right)\end{array}\right] \in C^{2}$, então

$$
\begin{aligned}
& \frac{d}{d t} V_{n}\left(U\left(t, U_{0}, V_{0}\right), V\left(t, U_{0}, V_{0}\right)\right)=\left(A_{n} U(t), V(t)\right) \\
& +2 b\|V(t)\|_{L_{d}^{2}}^{2}+2 b(\dot{V}(t), U(t)\rangle+(\dot{V}(t) \cdot V(t)\rangle-\left\langle\mathrm{f}\left(C^{-}(t)\right) \cdot \Gamma^{r}(t)\right) \\
& =\left\langle A_{n} U+\dot{V}, I^{r}\right\rangle+2 b\left\langle\dot{V^{r}}, U\right\rangle+2 b\left\|\boldsymbol{T}^{*}(t)\right\|_{L_{d}^{2}}^{2}-\left\langle\mathrm{f}\left(C^{-}\right) . \Gamma^{-}\right)
\end{aligned}
$$


Pela equação (4.3). obtemos

$$
\begin{array}{r}
\frac{d}{d t} V_{n}\left(U_{\eta, n}(t), \frac{d U_{\eta, n}}{d t}(t)\right)=\left\langle-2 \eta A_{n}^{\frac{1}{2}} \frac{d U_{\eta, n}}{d t}(t)\right. \\
\left.-2 a \frac{d U_{\eta, n}}{d t}(t)+\mathrm{f}\left(U_{\eta, n}(t)\right), \frac{d U_{\eta, n}}{d t}(t)\right\rangle+2 b\left\|\frac{d U_{\eta, n}}{d t}(t)\right\|_{L_{d}^{2}}^{2} \\
+2 b\left\langle\mathrm{f}\left(U_{\eta, n}(t)\right)-A_{n} U_{\eta, n}(t)-2 \eta A_{n}^{\frac{1}{2}} \frac{d U_{\eta, n}}{d t}(t)-2 a \frac{d U_{\eta, n}}{d t}(t), U_{\eta, n}(t)\right\rangle \\
-\left\langle\mathrm{f}\left(U_{\eta, n}(t)\right), \frac{d U_{\eta, n}}{d t}(t)\right\rangle=-2 \eta\left\|A_{n}^{\frac{1}{4}} \frac{d U_{\eta, n}}{d t}(t)\right\|_{0}^{2}+(2 b-2 a)\left\|\frac{d U_{\eta, n}}{d t}(t)\right\|_{0}^{2} \\
-4 a b\left\langle\frac{d U_{\eta, n}}{d t}(t), U_{\eta, n}(t)\right\rangle-2 b\left\|U_{\eta, n}(t)\right\|_{1}^{2}+2 b\left\langle\mathrm{f}\left(U_{\eta, n}(t)\right), U_{\eta, n}(t)\right\rangle \\
\left.+4 b \eta\left\|A_{n}^{\frac{1}{4}} \frac{d U_{\eta, n}}{d t}(t)\right\|_{L_{d}^{2}} \| A_{n}^{\frac{1}{4}} U_{\eta, n}(t)\right) \|_{L_{d}^{2}}
\end{array}
$$

Como $b \leq \frac{1}{8}$ e pela Desigualdade de Young, temos:

$$
\begin{array}{r}
4 b \eta\left\|A_{n}^{\frac{1}{4}} \frac{d U_{\eta, n}}{d t}(t)\right\|_{L_{d}^{2}}\left\|A_{n}^{\frac{1}{4}} U_{\eta, n}(t)\right\|_{L_{d}^{2}}-2 \eta\left\|A_{n}^{\frac{1}{4}} \frac{d U_{\eta, n}}{d t}(t)\right\|_{L_{d}^{2}}^{2} \\
\leq 2 b \eta\left\|A_{n}^{\frac{1}{4}} U_{\eta, n}(t)\right\|_{L_{d}^{2}}^{2} \leq 2 b \eta\left(1+\frac{2}{\delta}\right)\left\|U_{\eta, n}(t)\right\|_{H_{d}^{1}}^{2} \leq \eta K\left\|U_{\eta, n}(t)\right\|_{H_{d}^{1}}^{2}
\end{array}
$$

Então, por (4.10) e (4.7). temos de (4.9) que para $\eta \leq \eta_{0}$

$$
\frac{d}{d t} V_{n}\left(U_{\eta, n}(t): \frac{d U_{\eta, n}}{d t}(t)\right) \leq-\frac{5}{8} k_{0}\left(\left\|\frac{d U_{\eta, n}}{d t}(t)\right\|_{L_{d}^{2}}^{2}+\left\|U_{\eta, n}(t)\right\|_{H_{d}^{1}}^{2}\right)+2 b\left\langle\mathrm{f}\left(U_{\eta, n}(t)\right), U_{\eta, n}(t)\right\rangle
$$

para $\eta \leq \eta_{0}$ e $k_{0}=\inf \left\{\frac{a}{2} ; \frac{b}{2}\right\}$.

Agora, por (1.3), temos que existe um $c_{\delta / 4}>0$ tal que

$$
\begin{aligned}
f(v) v & \leq-\frac{\delta}{4} v^{2}+c_{\delta / 4} \\
\tilde{F}(v) & \leq-\frac{\delta}{4} v^{2}+c_{\delta / 4}
\end{aligned}
$$

Assim, por (4.12), temos que:

$$
2 b\left\langle\mathrm{f}\left(C_{\eta, n}^{\cdot}(t)\right), U_{\eta, n}(t)\right\rangle \leq-\frac{\delta}{4}\left\|U_{\eta, n}(t)\right\|_{L_{d}^{2}}^{2}+c_{\delta / 4}
$$

Portanto, por (4.14). temos de (4.11) que

$$
\begin{aligned}
\frac{d}{d t} l_{n}\left(U_{\eta, n}(t) \cdot \frac{d U_{\eta, n}}{d t}(t)\right) & =-\frac{5}{8} k_{0}\left(\left\|\frac{d U_{\eta, n}}{d t}(t)\right\|_{L_{d}^{2}}^{2}+\left\|C_{\eta, n}^{*}(t)\right\|_{H_{d}^{1}}^{2}\right)+c_{\delta / 4} \\
& \leq-\frac{1}{2} k_{0} \|\left(U_{\eta, n}(t), \frac{d U_{\eta, n}}{d t}(t) \|_{Y_{n}^{0}}^{2}+c_{\delta / 4}\right.
\end{aligned}
$$

Verificamos também que o funcional $V_{b}$ é limitado inferiormente. De fato, por (4.13) e por (4.5), temos

$$
V_{n}\left(U_{\eta, n}(t) . \frac{d C_{\eta, n}^{-}}{d t}(t)\right) \geq \frac{1}{4}\left(\left\|\frac{d U_{\eta, n}}{d t}(t)\right\|_{L_{d}^{2}}^{2}+\left\|C_{\eta, n}(t)\right\|_{H_{d}^{1}}^{2}\right)-c_{\delta / 4}
$$


Mostraremos que existe $K_{0}>0$ tal que para todo $\left(U_{0}, V_{0}\right)$ com $\left\|\left(U_{0}, V_{0}\right)\right\|_{Y_{n}^{0}} \leq r_{0}$ existe $t_{0}=t\left(r_{0}\right)$ tal que

$$
V_{n}\left(U_{\eta, n}(t), V_{\eta, n}(t)\right) \leq K_{0}
$$

para todo $t \geq t_{0}$ e para todo $n$. A demonstração deste fato é análoga a dada para o caso contínuo, observando que obtemos a uniformidade com relação ao parâmetro $n$. De fato, seja $\bar{r}$ suficientemente grande tal que $-\frac{1}{2} k_{0} \bar{r}^{2}+c_{\delta / 4}=-1$, observamos que $\bar{r}$ é escolhido independente de $n$. Assim, se $\left(\left\|\frac{d U_{\eta, n}}{d t}(t)\right\|_{L_{d}^{2}}^{2}+\left\|U_{\eta, n}(t)\right\|_{H_{d}^{1}}^{2}\right) \geq \bar{r}$,por (4.15) temos que $\frac{d}{d t} V_{n}\left(C_{\eta, n}(t), \frac{d U_{\eta, n}}{d t}(t)\right) \leq-1$.

Seja $M_{n}=\max \left\{V_{n}(U, V) ;\|U\|_{H_{d}^{1}}^{2}+\|V\|_{L_{d}^{2}}^{2} \leq \bar{r}\right\}$ e seja $M=\max \left\{M_{n}, n \in \mathbb{N}\right\}$. Observamos que $M<\propto$ pois $\vec{r}$ independe de $n$. De fato, pois

$$
\begin{gathered}
\left|V_{n}(U, V)\right| \leq \frac{1}{2}\|(U, V)\|_{Y_{n}^{0}}+2 b\|U\|_{0}\|V\|_{0}+\|\tilde{F}(U)\|_{0} \\
\leq \frac{1}{2}\|(U, V)\|_{Y_{n}^{0}}+2 b\|U\|_{0}\|V\|_{0}+M\left(\|U\|_{H_{d}^{1}}\right)\|U\|_{0}
\end{gathered}
$$

onde $M=\max \left\{|f(s)| ;|s| \leq\|U\|_{H_{d}^{1}}\right\}$.

E seja $C_{M}=\left\{(U, V) ; V_{n}(U, V) \leq M\right\}$. Por (4.16), temos que $C_{M}$ é limitado independente de $n$. E; por definição, que $B_{\vec{r}}(0) \subset C_{M}$.

Seja $B_{r_{0}}$, mostraremos que para cada $\left(U_{0}, V_{0}\right) \in B_{r_{0}}$ temos $T_{\eta, n}\left(t_{0}\right)\left(U_{0}, V_{0}\right) \in C_{M}$ para algum $t_{0}=t\left(U_{0}, V_{0}\right)$ e que $\sup \left\{t\left(U_{0}, V_{0}\right) ;\left(U_{0}, V_{0}\right) \in B_{r_{0}}\right\}<\infty$ independente de $n$. De fato, temos dois casos a considerar.

i)Se $\left(L_{0}, T_{0}\right) \in C_{M}$ então nada há a provar.

ii) Se $\left(U_{0}, I_{0}\right) \notin C_{M}$ então $\left(U_{0}, V_{0}\right) \notin B_{\bar{\tau}}(0)$ entāo $\frac{d}{d t} V_{n}\left(U(t) . \top^{\top}(t)\right) \leq-1$ para todo $t$ tal que $\left(U(t), \Gamma^{*}(t)\right) \notin B_{\bar{r}}(0)$, logo $V_{n}(U(t), V(t)) \leq V_{n}\left(U_{0}, V_{0}\right)-t$.

Assim, $V\left(L_{0} . I_{0}^{\circ}\right)>M$ então $V_{n}(U(t), V(t)) \leq K-t$, onde $K^{\circ}=\max \left\{T_{n}^{*}\left(U, \Gamma^{\top}\right):\left(U, \Gamma^{\circ}\right) \in\right.$ $\left.B_{r_{0}}\right\}$. Logo consideramos $t_{0}=K-M>0$ e obtemos $V_{n}(U(t), V(t)) \leq M$. Observamos que $t_{0}$ foi escolhido uniforme em $B_{r_{0}}(0)$ e que. como antes, $K$ é limitado em $n$ e portanto $t_{0}$ também é limitado com $n$.

Verificamos que $C_{M}$ é positivamente invariante sobre $T_{\eta, n}(t)$. De fato, suponha por absurdo que existe um último tempo, $\bar{t}$, tal que $V\left(T_{\eta, n}(\bar{t})\left(U_{0}, V_{0}\right)\right)=M, T_{\eta \cdot n}(t)\left(L_{0} . \Gamma_{0}\right) \in C_{M}$ para $t_{0} \leq t \leq \bar{t}$ e $T_{\eta, n}(t)\left(U_{0}, V_{0}\right) \notin C_{M}$ para $\bar{t}<t$, logo $T_{\eta, n}(t)\left(U_{0}, V_{0}\right) \notin B_{\bar{r}}(0)$. Assim. $\Gamma\left(T_{\eta, n}(t)\left(\tau_{0}^{-} . T_{0}\right)\right)<\Gamma^{\top}\left(T_{\eta, n}(\bar{t})\left(U_{0}, V_{0}\right)\right)=M$, absurdo.

Logo, para qualquer $B_{r_{0}}$ existe $t_{B}$ tal que $F\left(T_{\eta \cdot n}\left(C_{0}, T_{0}\right)\right) \leq M$ para todo $t \geq t_{B}$ e 
conseqüentemente, por (4.16)

$$
\left(\left\|\frac{d U_{\eta, n}}{d t}(t)\right\|_{L_{d}^{2}}^{2}+\left\|U_{\eta, n}(t)\right\|_{H_{d}^{1}}^{2}\right) \leq C_{0}
$$

para $t \geq t_{B}$

Corolário 4.1. Existe uma constante $K_{0}>0$, independente de $n$ e de $\eta$ tal que

$$
\|(U, V)\|_{Y_{n}^{\prime 0}} \leq K_{0}
$$

para todo $(U, V) \in \mathcal{A}_{\eta n}$ e $0 \leq \eta \leq \eta_{0}$ e $n \geq n_{0}$.

Proposição 4.1. Para qualquer $0 \leq \eta \leq \eta_{0}$ e $r_{0}>0$ existe uma constante $C_{0}^{*}\left(r_{0}\right)$ tal que, para qualquer solução $U_{\eta, n}(t)$ de (4.1), com $\left(\left\|U_{0}\right\|_{H_{d}^{1}}^{2}+\left\|V_{0}\right\|_{L_{d}^{2}}^{2}\right)^{\frac{1}{2}} \leq r_{0}$, temos

$$
\int_{0}^{\infty}\left\|\frac{d U_{\eta, n}}{d t}(s)\right\|_{L_{d}^{2}}^{2} d s \leq C_{0}^{*}\left(r_{0}\right)
$$

Demonstração: Para qualquer $\eta>0$. Considere o funcional de Lyapunov

$$
V(U, V)=\frac{1}{2}\|V\|_{L_{d}^{2}}^{2}+\frac{1}{2}\|U\|_{H_{d}^{1}}^{2}-(\bar{F}(U), \overline{1})
$$

onde $\vec{F}$ é dada como anteriormente.

Seja $U_{\eta, n}$ a solução de (4.1) com condição inicial $\left(U_{0}, V_{0}\right)$, então, de (4.9) com $b=0$, obtemos que

$$
\frac{d}{d t} V\left(C_{\eta, n}(t), \frac{d U_{\eta, n}}{d t}(t)\right) \leq-2 a\left\|\frac{d U_{\eta, n}}{d t}(t)\right\|_{L_{d}^{2}}^{2}-2 \eta\left\|A_{n}^{\frac{1}{4}} \frac{d U_{\eta, n}}{d t}(t)\right\|_{L_{d}^{2}}^{2} \leq-2 a\left\|\frac{d U_{\eta, n}}{d t}(t)\right\|_{L_{d}^{2}}^{2} .
$$

Logo,

$$
V\left(U_{\eta, n}(t), \frac{d U_{\eta, n}}{d t}(t)\right)-V\left(U_{0}, \dot{V_{0}}\right) \leq-2 a \int_{0}^{t}\left\|\frac{d U_{\eta, n}}{d t}(s)\right\|_{L_{d}^{2}}^{2} d s
$$

ou seja,

$$
\int_{0}^{t}\left\|\frac{d U_{\eta, n}}{d t}(s)\right\|_{L_{d}^{2}}^{2} d s \leq \frac{1}{2 a}\left(V\left(U_{0}, V_{0}\right)-V\left(U_{\eta, n}(t), \frac{d U_{\eta, n}}{d t}(t)\right)\right)
$$

Como,

i) $V\left(U, \Gamma^{\circ}\right) \geq c\left(\|U\|_{H_{d}^{1}}^{2}+\|V\|_{L_{d}^{2}}^{2}\right)-c^{\prime}$

ii) $V(U, V) \leq c_{1}\left(r_{0}\right)\left(\|U\|_{H_{d}^{1}}^{2}+\|V\|_{L_{d}^{2}}^{2}\right)$ :

Logo,

$$
\begin{array}{r}
\int_{0}^{t}\left\|\frac{d U_{\eta, n}}{d t}(s)\right\|_{L_{d}^{2}}^{2} d s \leq \frac{1}{2 a}\left(V\left(U_{0}, V_{0}\right)-V\left(U_{\eta, n}(t) \cdot \frac{d U_{\eta, n}}{d t}(t)\right)\right) \\
\leq \frac{1}{2 a}\left(V\left(U_{0}, V_{0}\right)-c\left(\left\|U_{\eta, n}(t)\right\|_{H_{d}^{1}}^{2}+\left\|\frac{d U_{\eta, n}}{d t}(t)\right\|_{L_{d}^{2}}^{2}\right)+c^{\prime}\right) \leq \frac{1}{2 a}\left(V\left(U_{0}^{-}, V_{0}\right)+c^{\prime}\right)
\end{array}
$$


E assim,

$$
\int_{0}^{\infty}\left\|\frac{d U_{\eta, n}}{d t}(s)\right\|_{L_{d}^{2}}^{2} d s=\lim _{t \rightarrow \infty} \int_{0}^{t}\left\|\frac{d U_{\eta, n}}{d t}(t)\right\|_{L_{d}^{2}}^{2} d s \leq \frac{1}{2 a}\left({ }^{-}\left(U_{0} \cdot V_{0}\right)+c^{\prime}\right)
$$

Por ii), temos

$$
\int_{0}^{\infty}\left\|\frac{d U_{\eta, n}}{d t}(s)\right\|_{L_{d}^{2}}^{2} d s \leq \frac{1}{2 a}\left(V\left(U_{0}, V_{0}\right)+c^{\prime}\right) \leq c_{1}\left(r_{0}\right)\left(\left\|U_{0}\right\|_{H_{d}^{1}}^{2}+\left\|V_{0}\right\|_{L_{d}^{2}}^{2}\right)+c^{\prime}=C_{0}^{*}\left(r_{0}\right)
$$

Além disso, por (i) temos

$$
\begin{array}{r}
\left(\left\|\frac{d U_{\eta, n}}{d t}(t)\right\|_{L_{d}^{2}}^{2}+\left\|U_{\eta, n}(t)\right\|_{H_{d}^{1}}^{2}\right) \leq \frac{1}{c}\left(\Gamma^{\prime}\left(L_{\eta, n}^{*}(t), \frac{d U_{\eta, n}}{d t}(t)\right)+c^{\prime}\right) \\
\quad \leq \frac{1}{c}\left(V\left(U_{0}, V_{0}\right)+c^{\prime}\right) \leq \frac{1}{c}\left(c_{1}\left(r_{0}\right)\left(\left\|V_{0}^{\prime}\right\|_{L_{d}^{2}}^{2}+\left\|U_{0}^{-}\right\|_{H_{d}^{1}}^{2}\right)+c^{\prime}\right)
\end{array}
$$

E assin, $\left(\left\|\frac{d U_{\eta, n}}{d t}(t)\right\|_{L_{d}^{2}}^{2}+\left\|U_{\eta, n}(t)\right\|_{H_{d}^{2}}^{2}\right) \leq C_{1}^{*}\left(r_{0}\right)$.

\subsubsection{Limitação uniforme em $n$ e em $\eta$ dos atratores $\mathcal{A}_{\eta n}$ na norma de $Y_{n}^{1}$}

Teorema 4.2. Sejam $0<\eta<\eta_{0}$ e $n \geq n_{0}$ então existe uma constante $C_{2}>0$ e para cada $r_{0}$ e $r_{1}$. constantes $C_{2}^{*}\left(r_{0}\right)>0$ e $C_{3}^{*}\left(r_{1}\right)>0$, tal que para toda solução $L_{\eta, n}^{*}(t)$ de (4.1). com $\left\|\left(U_{0} . V_{0}\right)\right\|_{Y_{n}^{0}}^{2} \leq r_{0}$ e $\left\|\left(U_{0}, V_{0}\right)\right\|_{Y_{n}^{1}}^{2} \leq r_{1}$.

$$
\left\|\frac{d^{2}}{d t^{2}} L_{\eta, n}(t)\right\|_{L_{d}^{2}}^{2}+\left\|\frac{d U_{\eta, n}}{d t}(t)\right\|_{H_{d}^{1}}^{2}+\left\|L_{\eta, n}(t)\right\|_{H_{d}^{2}}^{2} \leq C_{2}^{*}\left(r_{0}\right)+C_{3}^{*}\left(r_{1}\right) e^{-C_{2} t}
$$

para todo $t \geq 0$

Demonstração: Agora consideramos o seguinte problema.

$$
\begin{aligned}
& \ddot{W}_{n}+2 \eta A_{n}^{\frac{1}{2}} \dot{W}_{n}^{\prime}+2 a \dot{W}_{n}+A_{n} W_{n}=G\left(\tau_{n}(t), T_{n}(t)\right) \\
& H_{\eta, n}^{-}(0)=T_{\eta, n}(0) \text {, } \\
& \dot{V}_{\eta, n}(0)=-2 \eta A_{n}^{\frac{1}{2}} V_{\eta, n}(0)-2 a T_{\eta, n}(0)-A_{n} L_{\eta, n}(0)+\mathrm{f}\left(C_{\eta, n}^{\tau}(0)\right)
\end{aligned}
$$

onde $G\left(U^{\prime} \Gamma^{\prime}\right)=\left(f^{\prime}\left(U_{1}\right) \Gamma_{i}, f^{\prime}\left(U_{2}\right) V_{2}, \cdots, f^{\prime}\left(L_{n}^{*}\right) \Gamma_{n}^{r}\right)$. Como $G\left(L_{\eta_{i} n}^{-}(t) . \Gamma_{\eta, n}^{r}(t)\right):[0, \infty)$ $\rightarrow \mathbb{R}^{n}$ é de classe $C^{1}$ então para $\left(W_{\eta, n}^{*}(0) . \dot{W}_{\eta \cdot n}(0)\right)$ existe uma única solução. Mas, por unicidadc. $\frac{d L_{\eta, n}^{-}}{d t}(t)=I_{\eta, n}(t) \in C^{2}\left((0 . \infty), \mathbb{R}^{n}\right)$ e satisfaz $(4.21) \log 0 \Pi_{\eta, n}^{-}(t)=\frac{d L_{\eta, n}^{-}}{d t}(t)$. 
Novamente. fixamos uma constante $b>0$ tal que $b$ satisfaz o lema (4.1) e consideramos o seguinte funcional

$$
V(U, V)=\frac{1}{2}\left(A_{n} U, U\right\rangle+2 b(U, V\rangle+\frac{1}{2}(V, V\rangle
$$

Assim: temos, para $W_{\eta, n}$ solução de (4.21):

$$
\begin{array}{r}
\frac{d}{d t} V(W(t), \dot{W}(t))=-2 \eta\left\|A_{n}^{\frac{1}{4}} \dot{W}_{\eta, n}(t)\right\|_{L_{d}^{2}}^{2} \\
\left.+(2 b-2 a)\left\|\dot{W}_{\eta, n}(t)\right\|_{0}^{2}+\left(G\left(U_{\eta, n}(t), V_{\eta, n}(t)\right)\right), \dot{W}_{\eta, n}(t)\right\rangle \\
\left.+4 b \eta\left\|A_{n}^{\frac{1}{4}} \dot{V}_{\eta, n}(t)\right\|_{L_{d}^{2}} \| A_{n}^{\frac{1}{4}} W_{\eta, n}(t)\right)\left\|_{L_{d}^{2}}-4 a b\left(\dot{W}_{\eta, n}(t), W_{\eta, n}(t)\right\rangle-2 b\right\| W_{\eta, n}(t) \|_{1}^{2} \\
\left.+2 b\left\langle G\left(U_{\eta, n}(t), V_{\eta, n}(t)\right)\right), W_{\eta, n}(t)\right)
\end{array}
$$

Como $b \leq \frac{1}{8}$. pela Desigualdade de Young, temos:

$$
4 b \eta\left\|A_{n}^{\frac{1}{4}} \dot{\eta}_{\eta, n}^{\cdot}(t)\right\|_{L_{d}^{2}}\left\|A_{n}^{\frac{1}{4}} W_{\eta, n}(t)\right\|_{L_{d}^{2}}-2 \eta\left\|A_{n}^{\frac{1}{4}} \dot{W}_{\eta, n}^{r}(t)\right\|_{L_{d}^{2}}^{2} \leq \eta K\left\|W_{\eta, n}(t)\right\|_{H_{d}^{1}}^{2}
$$

então, por (4.7):

$$
\begin{aligned}
& \left.\frac{d}{d t} V^{r}(I), \dot{H}(t)\right) \leq-k_{0}\left(\left\|\dot{W}_{\eta, n}(t)\right\|_{0}^{2}+\left\|W_{\eta, n}(t)\right\|_{1}^{2}\right)+\eta K\left\|W_{\eta, n}(t)\right\|_{H_{d}^{1}}^{2} \\
& \left.\left.\quad+2 b\left(G\left(U_{\eta, n}(t), V_{\eta, n}(t)\right)\right), W_{\eta, n}(t)\right\rangle+\left\langle G\left(U_{\eta, n}(t), \digamma_{\eta, n}(t)\right)\right), \dot{W}_{\eta, n}(t)\right\rangle
\end{aligned}
$$

Logo, para $\eta \leq \eta_{0}$. por Schwarz e Young, temos

$$
\begin{array}{r}
\frac{d}{d t} V(W(t), \dot{W}(t)) \leq-\frac{k_{0}}{2}\left(\left\|\dot{W}_{\eta, n}(t)\right\|_{0}^{2}+\left\|H_{\eta, n}^{*}(t)\right\|_{1}^{2}\right) \\
+\frac{k_{0}}{2}\left\|\dot{W}_{\eta, n}^{r}(t)\right\|_{L_{d}^{2}}^{2}+\frac{1}{2 k_{0}}\left\|G\left(C_{\eta, n}^{*}(t), V_{\eta, n}(t)\right)\right\|_{L_{d}^{2}}^{2} \\
+\frac{k_{0}}{2}\left\|W_{\eta, n}(t)\right\|_{H_{d}^{1}}^{2}+\left\|G\left(U_{\eta, n}(t) . V_{\eta, n}^{\cdot}(t)\right)\right\|_{L_{d}^{2}}^{2} \frac{2 b^{2}}{\lambda_{1} k_{0}} \\
\leq-\frac{k_{0}}{4}\left(\left\|\dot{i}_{\eta, n}^{-}(t)\right\|_{0}^{2}+\left\|W_{\eta, n}^{*}(t)\right\|_{1}^{2}\right)+\left(\frac{1}{2 k_{0}}+\frac{2 b^{2}}{\lambda_{1} k_{0}}\right)\left\|G\left(L_{\eta, n}^{-}(t), V_{\eta, n}^{r}(t)\right)\right\|_{L_{d}^{2}}^{2}
\end{array}
$$

Como, $\left\|G\left(C_{\eta, n}^{-}(t) . V_{\eta, n}^{*}(t)\right)\right\|_{L_{d}^{2}}^{2} \leq C^{*}\left(r_{0}\right)\left\|V_{\eta, n}(t)\right\|_{L_{d}^{2}}^{2}$ e por (4.6). temos

$$
\begin{aligned}
& \frac{d}{d t} V\left(U^{-}(t), \dot{H}^{\prime}(t)\right) \leq-\frac{k_{0}}{3}\left(\frac{1}{2}\left\|\dot{W}_{\eta, n}^{r}(t)\right\|_{L_{d}^{2}}^{2}\right. \\
& \left.+2 b\left(\Pi_{\eta, n}^{\cdot}(t) . \dot{\eta}_{\eta, n}^{\cdot}(t)\right\rangle+\frac{1}{2}\left\|\zeta_{\eta, n}^{r}(t)\right\|_{H_{d}^{1}}^{2}\right)+\left(\frac{1}{k_{0}}+\frac{4 b^{2}}{\lambda_{1} k_{0}}\right) C\left(r_{0}\right)\left\|\gamma_{\eta, n}(t)\right\|_{L_{d}^{2}}^{2} \\
& -\frac{k_{0}}{3} V(W(t) \cdot \dot{W}(t))+\left(\frac{1}{k_{0}}+\frac{4 b^{2}}{\lambda_{1} k_{0}}\right) C\left(r_{0}\right)\left\|\zeta_{\eta, n}(t)\right\|_{L_{d}^{2}}^{2}
\end{aligned}
$$


Resolvendo a desigualdade diferencial, temos

$V\left(W(t): \dot{H}^{\prime}(t)\right) \leq e^{-\frac{k_{0}}{3} t} V(W(0), \dot{W}(0))+e^{-\frac{k_{0}}{3} t}\left(\frac{1}{2 k_{0}}+\frac{2 b^{2}}{\lambda_{1} k_{0}}\right) C^{*}\left(r_{0}\right) \int_{0}^{t}\left\|V_{\eta, n}(s)\right\|_{L_{d}^{2}}^{2} d s$

Portanto, pelas desigualdades (4.5) e (4.6), temos

$$
\begin{array}{r}
\left\|\dot{W}_{\eta, n}(t)\right\|_{L_{d}^{2}}^{2}+\left\|W_{\eta, n}(t)\right\|_{H_{d}^{1}}^{2} \leq 4 T^{*}\left(W^{*}(t), \dot{W}(t)\right) \\
\leq 4\left(e^{-\frac{k_{0}}{3} t} V(W(0), \dot{W}(0))+e^{-\frac{k_{0}}{3} t}\left(\frac{1}{2 k_{0}}+\frac{2 b^{2}}{\lambda_{1} k_{0}}\right) C^{*}\left(r_{0}\right) \int_{0}^{t}\left\|T_{\eta, n}(s)\right\|_{L_{d}^{2}}^{2} d s\right. \\
\leq 4\left(e^{-\frac{k_{0}}{3} t} \frac{3}{4}\left(\left\|\dot{W}_{\eta, n}(t)\right\|_{L_{d}^{2}}^{2}+\left\|\Psi_{\eta, n}^{*}(t)\right\|_{H_{d}^{1}}^{2}\right)\right. \\
+e^{-\frac{k_{0}}{3} t}\left(\frac{1}{2 k_{0}}+\frac{2 b^{2}}{\lambda_{1} k_{0}}\right) C^{*}\left(r_{0}\right) \int_{0}^{t}\left\|T_{\eta, n}(s)\right\|_{L_{d}^{2}}^{2} d s
\end{array}
$$

Pela Proposição 4.1, temos

$$
\begin{array}{r}
\left\|\dot{W}_{\eta, n}(t)\right\|_{L_{d}^{2}}^{2}+\left\|W_{\eta, n}(t)\right\|_{H_{d}^{1}}^{2} \leq 4 \Gamma^{-}(W(t), \dot{W}(t)) \\
\leq 3 e^{-\frac{k_{0}}{3} t}\left(\left\|\dot{W}_{\eta, n}(0)\right\|_{L_{d}^{2}}^{2}+\left\|W_{\eta, n}^{\gamma}(0)\right\|_{H_{d}^{\frac{1}{2}}}^{2}\right)+4 e^{-\frac{k_{0}}{3} t}\left(\frac{1}{2 k_{0}}+\frac{2 b^{2}}{\lambda_{1} k_{0}}\right) C^{*}\left(r_{0}\right) C^{*}{ }_{0}\left(r_{0}\right)
\end{array}
$$

De forma análoga ao caso contínuo temos

$$
\left\|U_{\eta, n}\right\|_{H_{d}^{2}}^{2} \leq c\left(\left\|\mathrm{f}\left(U_{\eta, n}\right)\right\|_{L_{d}^{2}}^{2}+\left\|\frac{d W_{\eta, n}}{d t}\right\|_{L_{d}^{2}}^{2}+\left\|\Gamma_{\eta, n}\right\|_{H_{d}^{1}}^{2}\right)
$$

Com isto, obtemos de (4.30) e da equação (4.1), que

$$
\begin{array}{r}
\left(\left\|W_{\eta, n}^{2}(t)\right\|_{H_{d}^{1}}^{2}+\left\|\frac{d W_{\eta, n}}{d t}(t)\right\|_{L_{d}^{2}}^{2}+\left\|C_{\eta, n}^{-}(t)\right\|_{H_{d}^{2}}^{2}\right) \\
\leq c\left\|\mathrm{f}\left(U_{\eta, n}\right)\right\|_{L_{d}^{2}}^{2}+(1+c)\left(\left\|W_{\eta, n}(t)\right\|_{H_{d}^{1}}^{2}+\left\|\frac{d \Pi_{\eta, n}^{-}}{d t}(t)\right\|_{L_{d}^{2}}^{2}\right) \\
\leq C_{4}^{*}\left(r_{0}\right)+(1+c) C_{3}^{*}\left(r_{0}\right)+(1+c) 3 e^{-\frac{k}{3} t}\left(\left\|W_{\eta, n}(0)\right\|_{H_{d}^{2}}^{2}+\left\|\frac{d \Pi_{\eta, d}^{*}}{d t}(0)\right\|_{L_{d}^{2}}^{2}\right)
\end{array}
$$

Lembrando que $\left\|\frac{d W_{\eta, n}}{d t}(t)\right\|_{L_{d}^{2}} \leq 2 \eta\left\|U_{0}\right\|_{H_{d}^{1}}+2 a\left\|V_{0}\right\|_{L_{d}^{2}}+\left\|L_{0}\right\|_{H_{d}^{2}}+\left\|\mathrm{f}\left(C_{0}\right)\right\|_{L_{d}^{2}}$ obtemos, de (4.31), que

$$
\left(\left\|W_{\eta, n}^{-}(t)\right\|_{H_{d}^{1}}^{2}+\left\|\frac{d W_{\eta, n}}{d t}(t)\right\|_{L_{d}^{2}}^{2}+\left\|U_{\eta, n}(t)\right\|_{H_{d}^{2}}^{2}\right) \leq C_{5}^{*}\left(r_{0}\right)+C_{6}^{*} e^{-\frac{k}{3} t} r_{1}
$$

Portanto, seguem os corolários:

Corolário 4.2. Seja $0<\eta \leq \eta_{0}$. O problema (4.1) é limitado dissipativo em $Y_{n}^{-1}$ : uniformemente em $\eta$ e em n, ou seja, existe um conjunto $B \in I_{n}^{-1}$. B limitado tal que para todo subconjunto $C \in Y_{n}^{1}$. C limitado existe $t_{1}=t\left(r_{0} . r_{1}\right)$ e tal que

$$
T_{\eta, n}(t)(C) \subset B: \text { para } t \leq t_{1} .
$$

Observamos que a limitação de $B$ e $t\left(r_{0}, r_{1}\right)$ independem de $n$. 
Corolário 4.3. Sejam n e $0<\eta \leq \eta_{0}$. Então existem constantes $C_{3}$ e $C_{4}$ tais que: i)para todo $(U, V) \in \mathcal{A}_{\eta n}$ temos

$$
\|U\|_{H_{d}^{2}}^{2}+\|V\|_{H_{d}^{1}}^{2} \leq C_{3}
$$

ii)para toda órbita $\left(U_{\eta, n}(t), \frac{d U_{\eta, n}}{d t}(t)\right) \in \mathcal{A}_{\eta n}$ temos

$$
\left\|\frac{d^{2} U_{\eta, n}}{d t^{2}}(t)\right\|_{L_{d}^{2}}^{2} \leq C_{4}
$$

\subsubsection{Semi-continuidade superior dos atratores $\mathcal{A}_{\eta n}$ em $\eta=0$, para cada $n$.}

Teorema 4.3. Para cada $n>0$, os atratores dos problemas (4.1) são semi-contínuos superiormente em $\eta=0$, ou seja, para cada $n$ e $\forall \epsilon>0, \exists \eta_{0}(n)$ tal que $\eta \leq \eta_{0}$ temos

$$
\delta_{Y^{0}}\left(\mathcal{A}_{\eta n}, \mathcal{A}_{n}\right) \leq \epsilon
$$

Demonstração: Pelo Lema 1.2, provar este teorema. é suficiente provar que:

Afirmação 2. Para cada $n$, seja $\eta_{k}$ uma sequência de números positivos tal que $\eta_{k} \rightarrow 0$ quando $k \rightarrow \infty$ e seja $\left(U_{k, n}(t), \dot{U}_{k, n}(t)\right)$ uma sequência de soluções de (4.1) com $\eta=\eta_{k} e$ $n$ e tal que $\left(C_{k, n}(t), \dot{U}_{k, n}(t)\right) \in \mathcal{A}_{\eta_{k} n}$ para todo $t \geq 0$. Então existe uma subsequência de $\eta_{k_{j}}$ tal que $\left(\bar{U}_{k, n}(0), \dot{U}_{k, n}(0)\right)$ converge para $(\bar{U}, \bar{V}) \in \mathcal{A}_{n}$ em $\mathbb{R}^{n} \times \mathbb{R}^{n}$.

Demonstraçāo da afirmação (2). Esta demonstração será feita para cada $n$ fixo, logo abandonaremos o índice $n$.

De fato: Sabemos por (4.1) que existe um conjunto $B$ limitado em $\mathbb{R}^{n} \times \mathbb{R}^{n}$ tal que

$$
\left(\bigcup_{\eta} \mathcal{A}_{\eta}\right) \cup \mathcal{A} \subset B
$$

Assim, $\cup_{t} \cup_{k} L_{k}(t)$ é um conjunto relativamente compacto de $\mathbb{R}^{n}$ (com norma $H^{1}$ discretizada) e a família de aplicações $U_{k}(t) \in C\left(\mathbb{R}, \mathbb{R}^{n}\right)$, é equicontínua de $\mathbb{R}$ em $\mathbb{R}^{n}$.

Seja $J_{m}, m \geq 0$. uma sequência de intervalos compactos de $\mathbb{R}$ tal que $J_{m} \subset J_{m+1} . m \geq 0$ e 
$\cup_{m} J_{m}=\mathbb{R}$. Pelo teorema de Arzelà-Ascoli, existe uma subsequência $U_{k_{0}}$ de $U_{k}$ tal que $U_{k_{0}}$ converge para $\overline{L^{i}}$ em $C\left(J_{0}: \mathbb{R}^{n}\right)$. Por indução, aplicando o teorema de Arzelà-.Ascoli à subsequencia $U_{k_{m}}$ extraimos uma subsequência $U_{k_{m+1}}$ que converge para $\overline{C^{\prime}}$ em $C\left(J_{m+1}, \mathbb{R}^{n}\right)$. Considerando a sequência diagonal, existe uma subsequência de números positivos $\eta_{j_{k}}$ de $\eta_{k}$ e a correspondente subsequência $U_{j_{k}}$ de $U_{k}$ que são soluções de (4.1) com $\eta=\eta_{j_{k}}$ e tal que $U_{j_{k}} \rightarrow \bar{U}$ em $C\left(J, \mathbb{R}^{n}\right)$ para todo intervalo $J$ compacto. Temos que $\bar{U} \in C\left(\mathbb{R}, \mathbb{R}^{n}\right)$ e $\sup _{t \in \mathbb{R}}\|\bar{U}(t)\|_{H_{d}^{1}} \leq K_{1}$. Também, $\left\|A_{n}^{\frac{1}{2}} \dot{U}_{j_{k}}(t)\right\|_{L_{d}^{2}}=\left\|\dot{U}_{j_{k}}(t)\right\|_{H_{d}^{1}} \leq \bar{K}$, para todo $t$ e para todo $\eta_{j_{k}}$. Portanto,

$$
\sup _{t \in \mathbb{R}}\left(2 \eta_{j_{k}}\left\|A_{n}^{\frac{1}{2}} \dot{U}_{j_{k}}(t)\right\|_{L_{d}^{2}}\right) \rightarrow 0
$$

quando $j_{k} \rightarrow \infty$.

Sabemos que, $\left\|\dot{U}_{j_{k}}(t)\right\|_{H_{d}^{1}} \leq K$ e $\left\|\frac{d^{2} U_{j_{k}}}{d t^{2}}(t)\right\|_{L_{d}^{2}} \leq K$, para todo $t \in \mathbb{R}$ e para todo $j_{k}$ assim, $\frac{d U_{j_{k}}}{d t}(t)$ é uma a família em $C\left(J, \mathbb{R}^{n}\right)$ e portanto $\overline{\left(\frac{\overline{d U_{j_{k}}}}{d t}\right)_{k}}$ é compacto em $C\left(J, \mathbb{R}^{n}\right)$. Logo. existe uma subsequência de $\frac{d U_{j_{k}}}{d t}$, que também será denotada por $\frac{d U_{j_{k}}}{d t}$ e tal que existe $\bar{l} \in C\left(J . \mathbb{R}^{n}\right)$ tal que $\frac{d U_{j_{k}}}{d t} \rightarrow \bar{V}$ em $C\left(J, \mathbb{R}^{n}\right)$, para todo compacto $J$. Pelo fato que $\frac{d}{d t}$ é um operador fechado e $U_{j_{k}} \rightarrow \tilde{U}$, então $\frac{d \bar{l}}{d t}=\bar{V}$ e $\sup _{t \in E}\left\{\|\tilde{\Gamma}(t)\|_{0}\right\} \leq \tilde{K}$.

Desde que $(\bar{U}(t), \bar{V}(t))$ é globalmente definida e globalmente limitada em $Y_{n}^{0}$. resta apenas mostrar que $(\bar{U}(t), \bar{V}(t))$ é solução de (4.2). De fato, como $\left(U_{k}(t) . I_{k}(t)\right)$ satisfaz a equação integral:

$$
\left(U_{k}(t), V_{k}(t)\right)=e^{C_{\eta_{k}} t}\left(U_{0 k}, V_{0 k}\right)+\int_{0}^{t} e^{C_{\eta_{k}}(t-s)}\left[\left(0, \mathrm{f}\left(U_{k}(s)\right)\right)+\left(0 .-2 \eta \cdot H_{n}^{\frac{1}{2}} I_{k}(s)\right)\right] d s .
$$

Desde que $\left(U_{0 k}, V_{0 k}\right) \rightarrow(\bar{U}(0), \bar{r}(0))$ em $H_{n}^{1} \times L_{n}^{2}=\mathbb{R}^{n} \times \mathbb{R}^{n}$ e

$$
\left\|e^{C_{\eta_{k}} t}\left(\left(U_{0 k}, V_{0 k}\right)-(\bar{U}(0), \bar{V}(0))\right)\right\|_{Y_{n}^{\prime 0}} \leq M e^{\omega t}\left\|\left(U_{0 k}, T_{0 k}\right)-(\bar{C}(0) \cdot \bar{V}(0))\right\|_{Y_{n}^{\circ 0}}
$$

então $\left\|e^{C_{\eta_{k}} t}\left(\left(U_{0 k}, V_{0 k}^{r}\right)-(\bar{U}(0), \bar{V}(0))\right)\right\|_{Y_{n}^{-0}} \rightarrow 0$ quando $k \rightarrow \infty$ e uniformemente para $t$ em compactos. Também $\left(0, \mathrm{f}\left(U_{k}(s)\right)\right) \rightarrow(0, \mathrm{f}(\bar{C}(s)))$ em $C\left(\mathbb{R} \mathbb{R}^{n} \times \mathbb{R}^{n}\right)$ e uniformemente para $s$ em compactos. E como observado anteriormente,

$$
\left(2 \eta_{j_{k}}\left\|A_{n}^{\frac{1}{2}} \overline{V_{j_{k}}}(s)\right\|_{L_{d}^{2}}\right) \rightarrow 0
$$

quando $j_{k} \rightarrow \infty$, uniformemente para todo $s$.

E assim, $e^{C_{\eta_{k}} t}\left(U_{0 k} \cdot \mathrm{I}_{0 k}\right)+\int_{0}^{t} e^{C_{\eta_{k}}(t-s)}\left[\left(0, \mathrm{f}\left(U_{k}(s)\right)\right)+\left(0 .-2 \eta \cdot \mathrm{H}_{n}^{\frac{1}{2}} \Gamma_{k}(s)\right)\right] d s$ converge para $e^{C_{\eta_{k}} t}\left(\bar{U}(0) \cdot \overline{\Gamma^{\prime}}(0)\right)+\int_{0}^{t} e^{C_{\eta_{k}}(t-s)}\left[(0, \mathrm{f}(\bar{U}(s)))+\left(0 .-2 \eta A_{n}^{\frac{1}{2}} \bar{\Gamma}(s)\right)\right] d s$ quando $k \rightarrow \infty$ mas

$$
e^{C_{\eta_{k}} t}\left(C_{0 k} . V_{0 k}\right)+\int_{0}^{t} e^{C_{\eta_{k}}(t-s)}\left[\left(0, \mathrm{f}\left(U_{k}(s)\right)\right)+\left(0:-2 \eta A_{n}^{\frac{1}{2}} \Gamma_{k}(s)\right)\right] d s=\left(U_{k}(t) \cdot I_{k}(t)\right)
$$


e $\left(U_{k}(t), V_{k}(t)\right) \rightarrow(\bar{U}(t), \bar{V}(t))$. Portanto

$$
(\bar{U}(t), \bar{V}(t))=e^{C_{n} t}(\bar{U}(0), \bar{V}(0))+\int_{0}^{t} e^{C_{n}(t-s)}\left[(0, \mathrm{f}(\bar{U}(s)))+\left(0,-2 \eta A_{n}^{\frac{1}{2}} \bar{V}(s)\right)\right] d s,
$$

e é solução de (4.2). Isto conclui a demonstração.

\subsection{Semi-continuidade inferior}

A demonstração da semi-continuidade inferior, em $\eta$ para cada $n$ fixo, é análoga ao caso contínuo. Todas as hipóteses dos teoremas 1.10, 1.11 e 1.12 são obtidas uniformes em $n$. Como já foi observado, na demonstração do teorema 1.10 é necessário a uniformidade em $n$ do tempo necessário para um certo decaimento do funcional energia sobre a variedade instável na vizinhança dos pontos de equilíbrio. Esta uniformidade são foi possível ser obtida e assim, a semi-continuidade inferior é obtida para cada $n$ fixado.

Começamos com a verificação da semi-continuidade inferior das variedades instáveis locais.

Os pontos de equilíbrio para os problemas (4.3) e (4.4) são os mesmos para qualquer valor do parâmetro $\eta>0$. Estes pontos de equilíbrio são dados por: $Y_{n}=\left(U_{n}, 0\right)$ onde $U_{n}$

satisfaz $A_{n} U_{n}+\mathrm{f}\left(U_{n}\right)=0$. Consideremos um ponto de equilíbrio $Y_{n}$. Para cada $\eta \geq 0$ e $Y_{n}$ sejam $S_{\eta}^{n}$ e $L_{\eta}^{n}$ as variedades estáveis e instáveis respectivamente, para o problema (4.3), linearizado em $Y_{n}$. Sejam $P_{\eta}^{n}$ e $Q_{\eta}^{n}$ as projeções espectrais sobre $U_{\eta}^{n}$ e $S_{\eta}^{n}$ respectivamente. E seja $W_{\eta}^{n}$ a variedade instável para o problema não linear. Existe uma vizinhança $N_{\eta}^{n}$ de $Y_{n}^{r}$ e um homeomorfismo, $\sigma_{\eta}^{n}: N_{\eta}^{n} \cap U_{\eta}^{n} \rightarrow N_{\eta}^{n} \cap W_{\eta}^{n}$.

Conforme Teorema (1.12) devemos mostrar que se

$$
C_{\eta_{, n} n}^{\prime}\left(\left(U_{n}\right)_{j}\right)=\left[\begin{array}{cc}
0 & I \\
-A_{n} & -2\left(\eta A_{n}^{1 / 2}+a\right)
\end{array}\right]+\left[\begin{array}{cc}
0 & 0 \\
\mathrm{f}^{\prime}\left(\left(U_{n}\right)_{j}\right) & 0
\end{array}\right],
$$

onde $\mathrm{f}^{\prime}\left(U_{n}\right)=\operatorname{diag}\left(f^{\prime}\left(\left(U_{n}\right)_{1}\right), \ldots, f^{\prime}\left(\left(U_{n}\right)_{n}\right)\right)$ então existem $\sigma_{-}<0$ e $\sigma_{+}>0$ tais que o espectro de $C_{t, n}^{\prime}\left(U_{n}\right) . \sigma\left(C_{\eta, n}^{\prime}\left(U_{n}\right)\right.$ satisfaz:

$$
\sigma\left(C_{\eta, n}^{\prime}\left(U_{n}\right) \bigcap\left[\sigma_{-,} \sigma_{+}\right]=\emptyset .\right.
$$

Observamos $\sigma_{-}$e $\sigma_{+}$independem de $n$. Vamos mostrar primeiro que os autovalores, $\mu_{\eta k}^{n}$ : de $C_{\eta, n}^{\prime}\left(U_{n}\right)$ que são complexos tem parte real menor ou igual a $-a$, ou seja, $\operatorname{Re}\left(\mu_{\eta k}^{n}\right) \leq-a$. 
De fato. se $\lambda$ é autovalor de $C_{\eta, n}^{\prime}\left(U_{n}\right)$ com respectiva autofunçāo $(U, V) \in \mathbb{R}^{n} \times \mathbb{R}^{n}$ entāo $\lambda$ e $\left(U, I^{-}\right)$derem satisfazer:

$$
\lambda^{2} U+2 a \lambda U+2 \eta \lambda A_{n}^{\frac{1}{2}} U-A_{n} U+\mathrm{f}^{\prime}\left(U_{n}\right) U=0
$$

Se $\lambda \in \mathbb{C}$ então fazemos o produto interno de (4.34) por $\bar{U}$ e considerando $U$ normalizado em $L_{d}^{2}$, obtemos:

$$
\lambda^{2}+2 a \lambda+2 \eta \lambda\left\|A_{n}^{1 / 4} U\right\|_{L_{d}^{2}}^{2}+\|U\|_{H_{d}^{1}}^{2}+\sum_{i=1}^{n} \frac{1}{n}\left(\mathbf{f}^{\prime}\left(U_{n}\right)_{i}\right)\left\|U_{i}\right\|^{2}=0
$$

separando a parte imaginária obtemos:

$$
2 \operatorname{Re}(\lambda) \operatorname{Im}(\lambda)+2 a \operatorname{Im}(\lambda)+2 \eta \operatorname{Im}(\lambda)\left\|A_{n}^{1 / 4} U\right\|_{L_{d}^{2}}^{2}=0
$$

Desde que assumimos que $\lambda \in \mathbb{C}$ então:

$$
\operatorname{Re}(\lambda)=-\eta\left\|A_{n}^{1 / 4} U\right\|_{L_{d}^{2}}^{2}-a \leq-a .
$$

Portanto: para mostrarmos que existe uma separação no espectro para todo valor dos parâmetros $\eta$ e $n$, basta nos preocuparmos com os autovalores reais de $C_{\eta, n}^{\prime}\left(L_{n}^{*}\right)$. Assim, voltamos a ( 4.35$)$, e consideramos apenas a parte real.

$$
\operatorname{Re}(\lambda)^{2}-\operatorname{Im}(\lambda)^{2}+2 a \operatorname{Re}(\lambda)+2 \eta \operatorname{Re}(\lambda)\left\|A_{n}^{1 / 4} L^{i}\right\|_{L_{d}^{2}}^{2}+\|U\|_{H_{d}^{1}}^{2}+\sum_{i=1}^{n} \frac{1}{n}\left(\mathrm{f}^{\prime}\left(U_{n}\right)\right)_{i}\left\|C_{i}^{-}\right\|^{2}=0
$$

Desde que estamos considerando apenas $\lambda \in \mathbb{R}$ então $\operatorname{Iml}(\lambda)=0$ e obtemos:

$$
\operatorname{Re}(\lambda)^{2}+2 a \operatorname{Re}(\lambda)+2 \eta \operatorname{Re}(\lambda)\left\|A_{n}^{1 / 4} U\right\|_{L_{d}^{2}}^{2}+\|U\|_{H_{d}^{1}}^{2}+\sum_{i=1}^{n} \frac{1}{n}\left(\mathrm{f}^{\prime}\left(U_{n}\right)\right)_{i}\left\|U_{i}\right\|^{2}=0 .
$$

Resolrendo como equação de $2^{\circ}$ em $\operatorname{Re}(\lambda)$ obtemos :

$$
\operatorname{Re}(\lambda)=-a-\eta\left\|A_{n}^{1 / 4} U\right\|_{L_{d}^{2}}^{2} \pm \sqrt{\left(a+\eta\left\|A_{n}^{1 / 4} U^{U}\right\|_{L_{d}^{2}}^{2}\right)^{2}-\left(\sum_{i=1}^{n} \frac{1}{n}\left(f^{\prime}\left(L_{n}^{*}\right)\right)_{i} U_{i}^{2}+!_{i} U^{\prime} \|_{H_{d}^{1}}^{2}\right)}
$$

e portanto. $|\lambda|=|\operatorname{Re}(\lambda)| \leq K$ para algum $K>0$ independente de $\eta>0$ e de $n$.

No Apéndice mostramos a separação do espectro para $\eta \leq \eta_{0}$ e para $n \geq n_{0}$ através da convergência dos autovalores. E assim, a hipótese (K2), do teorema (1.12) está satisfeito. 
Lema 4.2. Seja $Y_{n}=\left(U_{n}, 0\right)$ um ponto de equilibrio de (4.1), para todo $\eta \geq 0$ e para cada $n$ fixo. A constante de Lipschitz da aplicação $G: \mathbb{R}^{n} \times \mathbb{R}^{n} \rightarrow \mathbb{R}^{n} \times \mathbb{R}^{n}$ dada por

$$
G\left(\left[\begin{array}{l}
R \\
S
\end{array}\right]\right)=\left[\begin{array}{c}
0 \\
f(R)
\end{array}\right]-\left[\begin{array}{c}
0 \\
f\left(U_{n}\right)
\end{array}\right]-\left[\begin{array}{cc}
0 & 0 \\
f\left(U_{n}\right) & 0
\end{array}\right]\left[\begin{array}{c}
R-U_{n} \\
S
\end{array}\right],
$$

onde $f\left(U_{n}\right)=\operatorname{diag}\left(f^{\prime}\left(\left(U_{n}\right)_{1}\right), \ldots, f^{\prime}\left(\left(U_{n}\right)_{n}\right)\right)$. Então, a constante de Lipschitz de $G$ na bola de raio $\frac{1}{k}$ e centro $U_{n}$ em $Y_{n}^{0}$ vai para zero quando $k \rightarrow \infty$.

Demonstração: Sejam $\left(W_{1}, Z_{1}\right)$ e $\left(W_{2}, Z_{2}\right)$ pontos de $\mathbb{R}^{n} \times \mathbb{R}^{n}$ em $B_{\frac{1}{k}}\left(U_{n}\right)$, então, pelo Teorema do Valor Médio, temos:

$$
\begin{array}{r}
\left\|G\left(\mathrm{~W}_{1}, Z_{1}\right)-G\left(W_{2}, Z_{2}\right)\right\|_{Y_{n}^{0}}^{2}=\left\|F\left(W_{1}\right)-F\left(W_{2}\right)-F^{\prime}(U)\left(W_{1}-W_{2}\right)\right\|_{L_{d}^{2}}^{2} \\
=\sum_{i=1}^{n} \frac{1}{n}\left[\left(F\left(W_{1}\right)\right)_{i}-\left(F\left(W_{2}\right)\right)_{i}-\left(\mathrm{f}^{\prime}\left(\left(U_{n}\right)_{2}\right)\right)\left(W_{1}-W_{2}\right)_{i}\right]^{2} \\
\left.\leq \sum_{i=1}^{n} \frac{1}{n}\left[f^{\prime}\left(\left(W_{1}\right)_{i}-t_{i}\left(W_{1}-W_{2}\right)_{i}\right)-f^{\prime}\left(U_{i}\right)\right]^{2}\left(W_{1}-W_{2}\right)_{i}\right)^{2} \\
\leq K\left(\left\|W_{1}-W_{2}\right\|_{H_{d}^{1}}^{2}\right) \sum_{0}^{n} \frac{1}{n}\left(W_{1}-W_{2}\right)_{i}^{2} \\
\leq K\left(\left\|W_{1}-W_{2}\right\|_{H_{d}^{1}}^{2}\right)\left\|W_{1}-W_{2}\right\|_{L_{d}^{2}}^{2}
\end{array}
$$

onde $K\left(\left\|\Psi_{1}^{-}-W_{2}\right\|_{H_{d}^{1}}^{2}\right)$ vai para zero quando $\left\|W_{1}-W_{2}\right\|_{H_{d}^{1}}$ vai para zero.

Portanto, a hipótese (K1) do teorema (1.12) está satisfeita.

Também temos que

$$
\begin{gathered}
\left\|T_{C_{\eta, n}^{\prime}}(t)(U, V)\right\|_{Y_{n}^{0}} \leq K e^{-\gamma t}\|(U, V)\|_{Y_{n}^{\cdot 0}}, \text { para } t \geq 0 \text { e }(U, V) \in S_{\eta}^{n}, \\
\left\|T_{C_{\eta, n}^{\prime}}(t)(U, V)\right\|_{Y_{n}^{0}} \leq K e^{\gamma t}\|(U, V)\|_{Y_{n}^{0}}, \text { para } t \leq 0 \text { e }(U, V) \in U_{\eta}^{n},
\end{gathered}
$$

para algum $\gamma>0$ Logo, as hipóteses do teorema (1.12) estão satisfeitas e portanto,nós temos as hipóteses (J4), (J5) e (J6) do teorema (1.11) verificadas.

Vamos mostrar que as hipóteses (J1), (J2). e (J3) também estão satisfeitas.

Para a hipótese (J1), ramos fazer a seguinte separação do operador linear $C_{\eta \cdot n}((U, V))$ $+H\left(\left(U, I^{\circ}\right)\right)$.

$$
C_{\eta, n}\left[\begin{array}{l}
U \\
V
\end{array}\right]+H\left(\left[\begin{array}{l}
U \\
V
\end{array}\right]\right)=C_{n}\left[\begin{array}{l}
U \\
V
\end{array}\right]+\left[\begin{array}{c}
0 \\
-2 \eta A_{n}^{\frac{1}{2}} \mathrm{~V}+\mathrm{f}(U)
\end{array}\right]=C_{n}\left[\begin{array}{l}
U \\
V
\end{array}\right]+G\left(\left[\begin{array}{l}
U \\
V
\end{array}\right]\right)+H\left(\left[\begin{array}{l}
U \\
V
\end{array}\right]\right)
$$


onde $G\left(\left[\begin{array}{l}L^{-} \\ I^{-}\end{array}\right]\right)=\left[\begin{array}{c}0 \\ -2 \eta A_{n}^{\frac{1}{2}} V\end{array}\right]$ e $H\left(\left[\begin{array}{l}U \\ V\end{array}\right]\right)=\left[\begin{array}{c}0 \\ \mathrm{f}(U)\end{array}\right]$. Pela fórmula da variação das constantes temos:

$$
T_{\eta, n}(t)\left[\begin{array}{l}
U \\
\digamma
\end{array}\right]=e^{C_{n} t}\left[\begin{array}{l}
U \\
V
\end{array}\right]+\int_{0}^{t} e^{C_{n}(t-s)}\left(G\left(T_{\eta, n}(s)\left(\left[\begin{array}{l}
U \\
V
\end{array}\right]\right)\right)+H\left(T_{\eta, n}(s)\left[\begin{array}{l}
U \\
V
\end{array}\right]\right)\right) d s
$$

$\mathrm{e}$

$$
T_{n}(t)\left[\begin{array}{l}
U \\
V
\end{array}\right]=e^{C_{n} t}\left[\begin{array}{l}
U \\
V
\end{array}\right]+\int_{0}^{t} e^{C_{n}(t-s)} H\left(T_{n}(s)\left(\left[\begin{array}{l}
U \\
V
\end{array}\right]\right)\right) d s
$$

Assim, para $0 \leq t \leq \tau$, temos:

$$
\begin{array}{r}
\left\|T_{\eta, n}(t)\left(\left[\begin{array}{l}
U \\
V
\end{array}\right]\right)-T_{n}(t)\left(\left[\begin{array}{l}
U \\
V
\end{array}\right]\right)\right\|_{Y_{n}^{0}} \\
\leq \int_{0}^{t}\left\|e^{C_{n}(t-s)}\left(H\left(T_{\eta, n}(s)\left(\left[\begin{array}{l}
U \\
V
\end{array}\right]\right)\right)-H\left(T_{n}(s)\left(\left[\begin{array}{c}
U \\
V
\end{array}\right]\right)\right)\right)\right\| d s \\
+\int_{0}^{t}\left\|e^{C_{n}(t-s)} G\left(T_{\eta, n}(s)\left(\left[\begin{array}{l}
U \\
V
\end{array}\right]\right)\right)\right\| d s
\end{array}
$$

Usaremos que $e^{C_{n} t}$ é um semigrupo de contraçōes. Também temos que $G: \mathbb{R}^{n} \times \mathbb{R}^{n} \rightarrow$ $\mathbb{R}^{n} \times \mathbb{R}^{n}$ Lipschitz em limitados. Para $(U, V) \in \mathcal{A}_{n}$ temos que $\left\|\left(U, V^{V}\right)\right\|_{Y_{n}^{1}} \leq r_{1}$ e assim. pelo teorema (4.2), temos que $\left\|T_{\eta, n}(s)\left(\left[\begin{array}{l}U \\ V\end{array}\right]\right)\right\|_{Y_{n}^{1}} \leq K$ para todo $s \geq 0$, para todo $n$ e $(U . V) \in \mathcal{A}_{n}$ então, por (4.36), temos

$$
\begin{array}{r}
\left\|T_{\eta, n}(t)\left(\left[\begin{array}{l}
U \\
V
\end{array}\right]\right)-T_{n}(t)\left(\left[\begin{array}{l}
U \\
V
\end{array}\right]\right)\right\|_{Y_{n}^{0}} \\
\left.\leq \int_{0}^{t} L \| T_{\eta, n}(s)\left(\left[\begin{array}{l}
U \\
V
\end{array}\right]\right)-T_{n}(s)\left(\left[\begin{array}{l}
U \\
V
\end{array}\right]\right)\right) \| d s+2 \eta M K \tau
\end{array}
$$

Por Gronwall. temos

$$
\left\|T_{\eta, n}(t)\left(\left[\begin{array}{l}
U \\
I^{\prime}
\end{array}\right]\right)-T_{n}(t)\left(\left[\begin{array}{l}
U \\
V^{\prime}
\end{array}\right]\right)\right\|_{Y_{n}^{\circ 0}} \leq 2 \eta M K \tau e^{M L t} \leq 2 \eta M K \tau e^{M L \tau}
$$

e assim, (J1) esta verificada, independente de $n$.

Pelo Apéndice, já mostramos a convergência em $\eta$ e $n$. dos autovalores dos problemas linearizados então se os pontos de equilíbrio do problema (3.2) são hiperbólicos então os 
pontos de equilíbrio dos problemas (4.1) para $\eta \leq \eta_{0}$ e $n \geq n_{0}$, também são hiperbólicos. E assim. a hipótese (J2) também está verificada.

Resta apenas verificarmos a hipótese (J3) para que tenhamos a semi-continuidade inferior das variedades instáveis. Mas isto segue do fato que os autovalores e os autovetores convergem, em $\eta$ e $n$, e as projeções sobre as variedades instáveis lineares são sobre espaços de dimensão finita. E assim, também são uniformemente limitadas, em $\eta$ e $n$, as projeções sobre as variedades estáveis.

O teorema 1.11, nos dá a semi-continuidade inferior das variedades instáveis. Desde que $\tau$ na demonstração deste teorema pode ser escolhido independente de $n$ (ver observação no final da seção) e as outras hi póteses são uniformes em $n$ então a semi-continuidade inferior das variedades instáveis locais também é uniforme em $n$.

Vamos agora mostrar que os atratores globais, $\mathcal{A}_{\eta n}$, dos problemas (4.1) são semicontínuos inferiormente em $\eta=0, \mathcal{A}_{n}$.

Teorema 4.4. Os atratores globais, $\mathcal{A}_{\eta n}$, dos problemas (4.1) são semi-contínuos inferiormente em $\eta=0, \mathcal{A}_{n}$, para cada $n$.

Demonstração: As hipóteses $(H 1)_{0, n}$ e $(H 2)_{0, n}$ já foram verificadas na seção 1.4 .2 do capítulo 1. Quanto a hipótese $(H 3)_{0, n}$ temos por [FO] que os pontos de equilíbrio do problema parabólico são genericamente hiperbólicos logo são um número finito e portanto os pontos de equilíbrio do problema (4.2) também são genericamente hiperbólicos (com argumento de perturbação da não linearidade). A hipótese $(H 4)_{\eta, n}$ já foi verificada na seção (1.4.2) do capítulo 1. Como observado para a hipótese (J2), desde que $(H 3)_{0, n}$ é assegurada e temos a convergência, em $\eta$ e em $n$, de autovalores do problema linearizado para $\eta \rightarrow 0$ e $n \rightarrow \infty$ então $(H 5)_{\eta, n}$ é verificada. E a hipótese $(H 6)_{\eta}$ também já foi verificada anteriormente. Assim, resta apenas verificarmos a hipótese $(H 7)_{\eta, n}$. De fato, mostraremos que dados $\epsilon>0$ e $\tau>0$, existem um número real $\delta^{*}=\delta(\epsilon, \tau)>0$ e um $\eta_{0}>0$ tal que

$$
\left\|T_{\eta, n}(t)(U, V)_{\eta, n} T_{n}(t)(U, V)_{n}\right\|_{\leq \epsilon}
$$

para $0 \leq t \leq \tau,\left(U, V^{\top}\right)_{n} \in \mathcal{A}_{n},(U, V)_{\eta, n} \in \mathcal{A}_{\eta n},\left\|(U, V)_{n}-(U, V)_{\eta, n}\right\|_{Y_{n}^{0}} \leq \delta^{*}, \eta \leq \eta_{0} \mathrm{e}$ para todo $n \geq n_{0}$.

Pela hipótese (J1) e por $\left\|T_{\eta, n}(t)\left((U, V)_{\eta, n}\right)-T_{\eta, n}(t)\left(\left(U, V^{\circ}\right)_{n}\right)\right\| \leq e^{L t}\left\|(U, V)-\left(U . V^{V}\right)_{\eta, n}\right\|_{Y_{n}^{0}}$ 
temos

$$
\begin{array}{r}
\left\|T_{\eta, n}(t)\left((U, V)_{\eta, n}\right)-T_{n}(t)\left((U, V)_{n}\right)\right\|_{Y_{n}^{0}} \\
\leq\left\|T_{\eta, n}(t)\left(\left(\tau^{*} \top^{*}\right)_{\eta, n}\right)-T_{\eta, n}(t)\left((U, V)_{n}\right)\right\|_{Y_{n}^{0}}+\left\|T_{\eta, n}(t)\left(\left(U, Y^{-}\right)_{n}\right)-T_{n}(t)\left((U, V)_{n}\right)\right\|_{Y_{n}^{0}} \\
\leq e^{L t}\left\|(U, V)_{\eta, n}-(U, V)_{n}\right\|_{Y_{n}^{0}}+2 \eta M K \tau e^{M L \tau}
\end{array}
$$

Assim, considere $\delta^{*}$ tal que $e^{L \tau}\left\|(U, V)_{\eta, n}-(U, V)_{n}\right\|_{Y_{n}^{0}} \leq \frac{\epsilon}{2}$ e escolha $\eta_{0}$ tal que para $\eta \leq \eta_{0}$ e para todo $n \geq n_{0}$ temos $2 \eta M K \tau e^{M L \tau} \leq \frac{\epsilon}{2}$.

Assim, as hipóteses do teorema 1.10 estão verificadas e temos a semi-continuidade inferior dos atratores $\mathcal{A}_{\eta n}$ em $\eta=0$ para cada $n$ fixado.

Mostraremos agora a indepêdencia em $n$ de $\tau$ do Teorema 1.11.

De fato, seja $w(t)$ solução por $w_{0} \in W(\Phi)$ logo, $w(t)=(x(t), y(t))$ onde $x(t)=\sigma(y(t))$. Seguindo a notaçāo utilizada nos Teoremas 1.11 e teoremadeAlexandreeHines2; temos

$$
w(t)=T_{n}(t)\left(P_{n} w_{0}\right)+\int_{-\infty}^{t} T_{n}(t-s) g_{k}(x(s), y(s)) d s+\int_{0}^{t} T_{n}(t-s) h_{k}(x(s), y(s)) d s
$$

para $t \leq 0$. Adotamos o índice $k$ referente a bola de raio $1 / k$ considerada. Assim,

$$
\|w(t)\| \leq K p e^{\gamma t}\left\|w_{0}\right\|+K p L_{f_{k}} \int_{-\infty}^{0} e^{\gamma s}\|u(s+t)\| d s+K q L_{f_{k}} \int_{t}^{0} e^{\gamma(t-s)}\|w(s)\| d s .
$$

Portanto, temos

$$
\left\|u^{\prime}(t)\right\| \leq K^{\prime \prime} e^{\gamma t}+M^{\prime} \int_{-\infty}^{0} e^{\gamma s}\|u(s+t)\| d s+L^{\prime} \int_{t}^{0} e^{\gamma(t-s)}\|w(s)\| d s .
$$

Seja $\beta=\frac{L^{\prime}}{\gamma}+\frac{M^{\prime}}{r}$. Desde que $\beta \rightarrow 0$ pois $L_{f_{k}} \rightarrow 0$ quando $k \rightarrow \propto$. onde $L_{f_{k}}$ é a constante de Lipschitz de $f$ na bola de raio $1 / k$, então

$$
\|w(t)\| \leq(1-\beta)^{-1} K^{\prime} e^{-\left[\gamma-(1-\beta)^{-1} L^{\prime}\right]|t|}
$$

e para $k$ suficientemente grande temos que $\|w(t)\| \rightarrow 0$ quando $t \rightarrow-\infty$. A convergência acima é uniforme em $n$ pois as constantes $K, \gamma, p$ e $q$ são uniformemente limitadas em $n$. A desigualdade utilizada acima pode ser encontrada en [Ha3], pp 110.

Assim, $\tau$ pode ser escolhido independente de $n$ no Teorema 1.11 .

OBSERI AÇ. İO: Todas as hipóteses do Teorema 1.10 são verificadas com uniformidade em relação ao parâmetro n. Mas, pela observação no final da demonstração do Teorema 1.10 , é necessário a independência em $n$ do decaimento de $I_{n}$ num certo conjunto c isto não foi possível ser obtido. Assim, a semi-continuidade inferior está provada para cada $n$ fixo. 


\title{
Capítulo 5
}

\section{Semi-continuidade superior de}

\author{
atratores dos problemas da onda
}

com atrito forte e sua respectiva

equação discretizada

\section{Introdução}

Nesta parte do trabalho vamos mostrar a semi-continuidade superior dos atratores para os problemas

$$
\begin{aligned}
& u_{t t}+2 \eta A^{1 / 2} u_{t}+2 a u_{t}=-A u+f(u), 0<x<1: t>0 \\
& u_{x}(0)=u_{x}(1)=0, t>0
\end{aligned}
$$

e

$$
\ddot{U}+2 \eta A_{n}^{1 / 2} \dot{U}+2 a \dot{U}=-A_{n} U+\mathrm{f}(U)
$$

onde $\eta>0, a>0, A u=-u_{x x}+\frac{\delta}{2} u, A_{n}$ é a matriz $n \times n$ dada por $A_{n}=\Delta_{n}+\frac{\delta}{2} I$ e $\Delta_{n}$ é dada por $(1.10), \mathrm{f}(U)=\left(f\left(u_{1}\right), \cdots, f\left(u_{n}\right)\right)^{\top}$ e $U=\left(u_{1}, \cdots, u_{n}\right)^{-}$.

Como no Capítulo 1, trabalharemos com as equações acima. respectivamente nas seguintes formas:

$$
\frac{d}{d t}\left[\begin{array}{l}
u \\
v
\end{array}\right]=C_{\eta}\left[\begin{array}{l}
u \\
v
\end{array}\right]+h\left(\left[\begin{array}{l}
u \\
v
\end{array}\right]\right)
$$


e

$$
\frac{d}{d t}\left[\begin{array}{l}
U \\
V
\end{array}\right]=C_{\eta n}\left[\begin{array}{l}
U \\
V
\end{array}\right]+H\left(\left[\begin{array}{l}
U \\
V
\end{array}\right]\right)
$$

onde

$$
C_{\eta}=\left[\begin{array}{cc}
0 & I \\
-A & -2\left(\eta A^{1 / 2}+a\right)
\end{array}\right] \text { e } h\left(\left[\begin{array}{l}
u \\
v
\end{array}\right]\right)=\left[\begin{array}{c}
0 \\
f^{e}(u)
\end{array}\right]
$$

$D\left(C_{\eta}\right)=X^{1} \times X^{\frac{1}{2}}$

$$
C_{\eta n}=\left[\begin{array}{cc}
0 & I_{n} \\
-A_{n} & -2\left(\eta A_{n}^{1 / 2}+a\right)
\end{array}\right] \text { e } H\left(\left[\begin{array}{l}
U \\
V
\end{array}\right]\right)=\left[\begin{array}{c}
0 \\
\mathrm{f}(U)
\end{array}\right]
$$

Pelos resultados do Capítulo 1. temos a existência de um atrator global $\mathcal{A}_{\eta}$ para (5.1) e um atrator global $\mathcal{A}_{\eta n}$ para (5.2).

A demonstração da semi-continuidade superior requer a imersão do espaço de fase do problema discreto no espaço de fase do problema contínuo. Observamos que a técnica da redução através da variedade invariante utilizada no problema escalar parabólico não pode ser utilizada pois neste caso nāo temos a existência de gap no espectro do operador linear do problema, pois $\lim _{k \rightarrow \infty} \operatorname{Re}\left(\lambda_{ \pm(k+1)}\right)-\operatorname{Re}\left(\lambda_{ \pm k}\right)=\eta$, onde $\lambda_{k}$ é o $k$-ésimo autovalor de $C_{\eta}$

Para resolvermos este problema com $\eta>0$ fixado, procedemos da seguinte forma: primeiramente fazemos a comparação dos problemas lineares. ou seja. mostramos a proximidade dos semigrupos lineares, e para isso separamos o problema em duas partes, onde em uma parte utilizamos que $\operatorname{Re}\left(\lambda_{ \pm k}\right) \rightarrow-\infty$, quando $k \rightarrow \infty$ nos restando comparar os semigrupos em um espaço de dimensāo finita e nesta parte. utilizamos a convergência dos autovalores e autovetores do problema discreto para o problema contínuo. Além disso, é necesário que façamos a comparação para condiçōes iniciais dos tipos $i\left(U_{0}, V_{0}\right)$ para $\left(U_{0}, V_{0}\right) \in \mathcal{A}_{\eta n}$ e também para $\left(u_{0}, v_{0}\right) \in C^{\mathrm{I}+\alpha} \times C^{\alpha}$ pois depois fazemos a comparação os semigrupos não lineares. Finalmente inostramos a semi-continuidade superior dos atratores. Esta técnica é utilizada em [ACR2].

A fim de fazermos a imersão de $\mathbb{R}^{n} \times \mathbb{R}^{n}$ em $Y^{\prime 0}$ definimos a seguinte aplicação inclusão: Sejam $(U, V)^{T} \in \mathbb{R}^{n} \times \mathbb{R}^{n}$ ou seja. $\left(u_{1}, u_{2}, \cdots, u_{n}, v_{1}, v_{2}, \cdots, v_{n}\right)^{T} \operatorname{definimos} i(U, V)=$ $(u(x), v(x))$ onde $u(x)$ e $v(x)$ são dadas por 


$$
u(x)=u_{1} \chi_{\left[0, \frac{1}{2 n}\right)}+\sum_{i=1}^{n_{1}}\left(u_{i}+\left(u_{i+1}-u_{i}\right) n\left(x-x_{i}\right)\right) \chi_{\left[\frac{2 i-1}{2 n} \cdot \frac{21+1}{2 n}\right)}+u_{n} \chi_{\left[\frac{2 n-1}{2 n}, 1\right]}
$$

e

$$
v(x)=\sum_{i=1}^{n} v_{i} \chi_{I_{i}}
$$

onde nós denotamos por $I_{i}$ O intervalo $\left[\frac{i-1}{n}, \frac{i}{n}\right)$

Teorema 5.1. A aplicação inclusão, $i: \mathbb{R}^{n} \times \mathbb{R}^{n} \rightarrow H^{1} \times L^{2}$ é contínua.

Demonstração: De fato:

$$
\begin{aligned}
\|u(x)\|_{L^{2}}^{2} & =\frac{u_{1}^{2}}{2 n}+\sum_{j=1}^{n-1} \int_{x_{j}}^{x_{j+1}} u^{2}(x) d x+\frac{u_{n}^{2}}{2 n} \\
& \leq \frac{u_{1}^{2}}{2 n}+\sum_{j=1}^{n-1}\left(\frac{u_{j+1}^{2}}{2 n}+\frac{u_{j}^{2}}{2 n}\right)+\frac{u_{n}^{2}}{2 n}=\sum_{j=1}^{n} \frac{u_{j}^{2}}{n}=\|U\|_{L_{d}^{2}}^{2}
\end{aligned}
$$

também,

$$
\|u(x)\|_{H^{1}}^{2}=\sum_{j=1}^{n-1} n\left(u_{j+1}-u_{j}\right)^{2}=\|U\|_{H_{d}^{1}}^{2}
$$

e $\|v(x)\|_{L^{2}}^{2}=\sum_{j=1}^{n} \frac{1}{n} v_{i}^{2}=\|V\|_{L_{d}^{2}}^{2}$. Assim,

$$
\left\|i\left(L^{\cdot} \cdot V^{\prime}\right)\right\|_{\mathbb{R}^{n} \times \mathbb{R}^{n}}=\left(\|u(x)\|_{H^{1}}^{2}+\frac{\delta}{2}\|u(x)\|_{L^{2}}^{2}+\|v(x)\|_{L^{2}}^{2}\right)^{\frac{1}{2}} \leq \|\left(L^{*} \cdot V^{\prime}\right){\| \mathbb{R}^{n} \times \sum^{n}}
$$

Também para sermos capazes de comparar os semigrupos definimos uma projeção de $\mathrm{Y}^{\circ 0}$ sobre $\mathbb{R}^{n} \times \mathbb{R}^{n}$ da seguinte forma. Consideramos a base de $\mathrm{I}^{\circ}$ é dada por, se os autovalores $\lambda_{ \pm k}$ de $C_{\eta}$ são reais entāo $\phi_{ \pm k}$ é o autovetor correspondente: se os autovalores $\lambda_{ \pm k}$ são complexos então consideramos os vetores $\psi_{+k}=\operatorname{Re}\left(o_{=k}\right)$ e $\psi_{-k}=\operatorname{Im}\left(\phi_{+k}\right)$, onde $\phi_{ \pm k}=\left[\begin{array}{c}e_{k} \\ \lambda_{ \pm k} e_{k}\end{array}\right]$ e $e_{k}$ é autovetor de $A$. Para cada $e_{k}$ definimos $P_{n}\left(e_{k}\right)=U \in \mathbb{R}^{n}$ dado por $U^{*}=\left(u_{1}, u_{2}, \cdots, u_{n}\right)$ onde cada $u_{i}$ é $e_{k}\left(x_{\imath}\right)$. Esta definição pode ser dada para qualquer função contínua. Portanto definimos $P_{2 n}\left(\phi_{ \pm k}\right)=\left(P_{n}\left(e_{k}\right) \cdot \lambda_{ \pm k} P_{n}\left(e_{k}\right)\right)$ e $P_{2 n}\left(\psi_{+k}\right)=\left(P_{n}\left(e_{k}\right), \operatorname{Re}\left(\lambda_{ \pm k}\right) P_{n}\left(e_{k}\right)\right)$ e $P_{2 n}\left(\psi_{-k}\right)=\left(P_{n}\left(e_{k}\right) \cdot \operatorname{Im}\left(\lambda_{-k}\right) P_{n}\left(e_{k}\right)\right)$ estendemos por linearidade sobre $Y^{0}$.

Teorema 5.2. A aplicação projeção, $P_{2 n}$, é contínua, com constante de continuidade independente de $n$. 
Demonstração: De fato, como $P_{n}(\cos (k \pi x))=U=\left(\cos \left(k \pi x_{1}\right), \ldots, \cos \left(k \pi x_{1}\right)\right)$ então temos:

$$
\begin{aligned}
\|U\|_{H_{d}^{1}}^{2} & =\sum_{1}^{n-1} n\left(u_{i+1}-u_{i}\right)^{2}=\sum_{1}^{n-1} n\left(\cos \left(k \pi x_{i+1}\right)-\cos \left(k \pi x_{i}\right)\right)^{2} \\
& =\sum_{1}^{n-1} n(k \pi)^{2} \operatorname{sen}^{2}\left(k \pi \bar{x}_{i}\right) \frac{1}{n^{2}} \leq(k \pi)^{2} \leq 2\|k \pi \operatorname{sen}(k \pi x)\|_{L^{2}}^{2}
\end{aligned}
$$

e

$$
\|U\|_{L_{d}^{2}}^{2}=\sum_{1}^{n} \frac{1}{n} u_{i}^{2}=\sum_{1}^{n} \frac{1}{n} \cos ^{2}\left(k \pi x_{i}\right) \leq 1=2\|\cos (k \pi x)\|_{L^{2}}^{2}
$$

Logo,

$$
\begin{array}{r}
\left\|P_{2 n}\left(\phi_{ \pm k}\right)\right\|_{H_{d}^{1} \times L_{d}^{2}}=\left\|\left(P_{n}\left(e_{k}\right), \lambda_{ \pm k} P_{n}\left(e_{k}\right)\right)\right\|_{H_{d}^{1} \times L_{d}^{2}} \\
=\left(\left\|P_{n}\left(e_{k}\right)\right\|_{H_{d}^{1}}^{2}+\frac{\delta}{2}\left\|P_{n}\left(e_{k}\right)\right\|_{L_{d}^{2}}^{2}+\left\|\left|\lambda_{ \pm k}\right|\right\| P_{n}\left(e_{k}\right) \|_{L_{d}^{2}}^{2}\right)^{\frac{1}{2}} \\
\leq\left(2\left\|e_{k}\right\|_{H^{1}}+\frac{\delta}{2} 2\left\|e_{k}\right\|_{L^{2}}+\left|\lambda_{ \pm k}\right| 2\left\|e_{k}\right\|_{L^{2}}^{2}\right)^{\frac{1}{2}} \\
=\sqrt{2}\left\|\phi_{ \pm k}\right\|_{H^{1} \times L^{2}}
\end{array}
$$

Outro resultado que temos é

Teorema 5.3. i) Sejam $\lambda_{ \pm k}$ os autovalores de $C_{\eta}$ e $\lambda_{ \pm k}^{n}$ os autovalores de $C_{\eta n}$ então para cada $k$ fixado temos que $\lambda_{ \pm k}^{n} \rightarrow \lambda_{ \pm k}$ quando $n \rightarrow \infty$.

ii) Sejam $\phi_{ \pm k}$ os autovetores de $C_{\eta}$ e $\phi_{ \pm k}^{n}$ os autovetores de $C_{\eta n}$ então para cada $k$ fixado temos que $i\left(\phi_{ \pm k}^{n}\right) \rightarrow \phi_{ \pm k}$ quando $n \rightarrow \infty$.

\subsection{Comparação dos Semigrupos Lineares}

Sejam $e^{C_{\eta} t}$ e $e^{C_{\eta n} t}$ os semigrupos gerados por $C_{\eta}$ e $C_{\eta n}$ respectivamente. Mostraremos que $\forall \epsilon>0, \exists n_{o}(\epsilon)$ tal que $\forall n \geq n_{0}$ temos

$$
\left\|e^{C_{\eta} t}\left(u_{0}, v_{0}\right)-i\left(e^{C_{\eta n} t} P_{2 n}\left(u_{0}, v_{0}\right)\right)\right\|_{H^{2} \times L^{2}} \leq \epsilon t^{-\alpha}\left\|\left(u_{0}, v_{0}\right)\right\|_{C^{1-o} \times C^{\circ}} . t>0
$$

para todo $\left(u_{0}, v_{0}\right) \in C^{1+a} \times C^{\alpha} \mathrm{e}$

$$
\left\|e^{C_{\eta} t} i\left(U_{0}, V_{0}\right)-i\left(e^{C_{\eta n} t}\left(U_{0}, V_{0}\right)\right)\right\|_{H^{1} \times L^{2}} \leq \epsilon t^{-\alpha}\left\|\left(U_{0}, V_{0}\right)\right\|_{\left(H^{2} \times H^{1}\right)_{d}}, t>0
$$

para todo $\left(U_{0}, V_{0}\right) \in \bigcup_{n} \mathcal{A}_{\eta n}$. 
Demonstração: Faremos a demonstração para a primeira desigualdade e quando necessário a distinção para o segundo caso.

Seja $\epsilon>0$. Dividiremos a demonstração em duas partes.

i) Para $0<t \leq \epsilon$.

$$
\begin{aligned}
\left\|e^{C_{\eta} t}\left(u_{0}, v_{0}\right)-i\left(e^{C_{\eta n} t} P_{2 n}\left(u_{0}, v_{0}\right)\right)\right\|_{H^{1} \times L^{2}} & \leq K^{\prime} e^{-\delta / 2 t}\left\|\left(u_{0}, v_{0}\right)\right\|_{H^{1} \times L^{2}} \\
& \leq M \epsilon^{\nu} t^{-\alpha}\left\|\left(u_{0}, v_{0}\right)\right\|_{H^{1} \times L^{2}}, \alpha>\nu>0 .
\end{aligned}
$$

ii) Para $t>\epsilon$. Seja $\beta \in(0,1)$ um número fixado. Desde que $\lambda_{ \pm k}^{n} \rightarrow \lambda_{ \pm k}$ quando $n \rightarrow \infty$ e $\operatorname{Re}\left(\lambda_{ \pm k}\right) \rightarrow-\infty$, quando $k \rightarrow \infty$ então existem $K(\epsilon)$ e $N(\epsilon)$ tal que $e^{\operatorname{Re}\left(\lambda_{ \pm k}^{n}\right) t} \leq \epsilon t^{-\beta}$, para todo $n \geq N(\epsilon)$ e $k \geq K(\epsilon)$, e $e^{R e\left(\lambda_{ \pm k}^{n}\right) t} \leq \epsilon t^{-\beta}$ para todo $k \geq K(\epsilon)$.

Queremos estimar

$$
\left\|e^{C_{\eta} t}\left(u_{0}, v_{0}\right)-i\left(e^{C_{\eta^{n}} t} P_{2 n}\left(u_{0}, v_{0}\right)\right)\right\|_{H^{1} \times L^{2}}
$$

para $t \geq \epsilon$.

Veste caso faremos a decomposição dos espaços $H^{1} \times L^{2}$ e $\mathbb{R}^{n} \times \mathbb{R}^{n}$ da seguinte forma. Em $H^{1} \times L^{2}$ escrevemos $H^{1} \times L^{2}=\oplus E_{k}$ onde $E_{k}$ é o auto-espaço 2-dimensional real associado aos autovalores $\lambda_{ \pm k}$. Se $\lambda_{ \pm k}$ é real então $E_{k}=\left[\phi_{+k}, \phi_{-k}\right]$. Se $\lambda_{ \pm k}$ é complexo, consideraremos como base de $E_{k}$ os vetores $\psi_{+k}=\operatorname{Re} \phi_{+k}$ e $\tau_{-k}=\operatorname{Im} \phi_{+k}$.

Observamos que $\cos \prec\left(\psi_{+k}, \psi_{-k}\right)<1-\xi$, para algum $\xi>0$ e para todo $k$ pois

$$
\cos \prec\left(\psi_{+k} \cdot \dot{\psi}_{-k}\right)=\frac{\left\|e_{k}\right\|_{H^{1}}^{2}+\operatorname{Re}\left(\lambda_{+k}\right) \operatorname{Im}\left(\lambda_{+k}\right)}{\sqrt{\left\|e_{k}\right\|_{H^{1}}^{4}+\left(\operatorname{Re}^{2}\left(\lambda_{+k}\right)+\operatorname{Im}^{2}\left(\lambda_{+k}\right)\right)\left\|e_{k}\right\|_{H^{1}}^{2}+\left(\operatorname{Re}\left(\lambda_{+k}\right)\right)^{2}\left(\operatorname{Im}\left(\lambda_{+k}\right)\right)^{2}}}
$$

estamos considerando $\left\|e_{k}\right\|_{L^{2}}=1$ e lembrando que $\operatorname{Re}\left(\lambda_{+k}\right) \sim-\eta\left\|e_{k}\right\|_{H^{1}}$ e $\operatorname{Im}\left(\lambda_{+k}\right) \sim$ $\left(1-\eta^{2}\right)^{\frac{1}{2}}\left\|\epsilon_{k}\right\|_{H^{1}}$ então,

$$
\lim _{k \rightarrow \infty} \cos ^{2} \prec\left(\psi_{+k}, \psi_{-k}\right) \leq \frac{\left(1+\eta\left(1-\eta^{2}\right)^{\frac{1}{2}}\right)^{2}}{2+\eta\left(1-\eta^{2}\right)^{\frac{1}{2}}} \leq 1-\xi
$$

para algum $\xi>0$. Isto nos dá equivalência entre as normas da soma, do máximo e do produto interno em cada subespaço $E_{k}$, com constante de equivaléncia independente de $k$.

Assinl. escrevemos $(u, v) \in H^{1} \times L^{2}$ da seguinte forma

$$
\left(u \cdot v^{\prime}\right)=\sum_{k=1}^{K^{\prime}}\left((u, v)_{k}^{+} \phi_{+k}+(u, v)_{k}^{-} \phi_{-k}\right)+\sum_{k=K^{\prime}+1}^{\infty}\left((u, v)_{k}^{+} v_{+k}+(u, v)_{k}^{-} \imath^{-}-k\right)
$$


onde $(u, v)_{k}$ é a projeção de $(u, v)$ no espaço $E_{k}$ e $\|(u, v)\|=\left(\sum_{k=1}^{\infty}\left\|(u, v)_{k}\right\|^{2}\right)^{1 / 2}$.

Analogamente, em $\mathbb{R}^{n} \times \mathbb{R}^{n}$ escrevemos $\mathbb{R}^{n} \times \mathbb{R}^{n}=\oplus E_{k}^{n}$ é um subespaço 2 dimensional associado aos autovalores $\lambda_{ \pm k}^{n}$. Se $\lambda_{ \pm k}^{n}$ são autovalores reais então $E_{k}^{n}=\left[\phi_{+k}^{n}, \phi_{-k}^{n}\right]$, onde $\phi_{ \pm k}^{n}$ é o autovetor normalizado associado a $\lambda_{ \pm k}^{n}$. Se $\lambda_{ \pm k}^{n}$ é complexo consideraremos a base de $E_{k}^{n}$ como os vetores $\psi_{+k}^{n}=\operatorname{Re} \phi_{+k}^{n}$ e $\psi_{-k}^{n}=\operatorname{Im} \phi_{+k}^{n}$.

Assim, escrevemos $(U, V) \in \mathbb{R}^{n} \times \mathbb{R}^{n}$ da seguinte forma

$$
(U, V)=\sum_{k=1}^{K}\left((U, V)_{k}^{+} \phi_{+k}^{n}+(U, V)_{k}^{-} \phi_{-k}^{n}\right)+\sum_{k=K+1}^{n}\left((U, V)_{k}^{+} \psi_{+k}^{n}+(U, V)_{k}^{-} \psi_{-k}^{n}\right)
$$

onde $\left(U, V^{\top}\right)_{k}$ é a projeção de $(U, V)$ no espaço $E_{k}^{n}$ e $\|(U, V)\|=\left(\sum_{k=1}^{n}\left\|\left(U, \Gamma^{\top}\right)_{k}\right\|^{2}\right)^{1 / 2}$.

Agora voltamos a estimativa de $\left\|e^{C_{\eta} t}\left(u_{0}, v_{0}\right)-i\left(e^{C_{\eta n} t} P_{2 n}\left(u_{0}, v_{0}\right)\right)\right\|_{H^{1} \times L^{2}}$ para $t \geq \epsilon$. Então,

$$
\begin{array}{r}
\left\|e^{C_{\eta} t}\left(u_{0}, v_{0}\right)-i\left(e^{C_{\eta n} t} P_{2 n}\left(u_{0}, v_{0}\right)\right)\right\|_{H^{1} \times L^{2}} \\
\leq\left\|e^{C_{\eta} t} \sum_{k=1}^{K}\left(u_{0}, v_{0}\right)_{k}-i\left(e^{C_{\eta n} t} \sum_{k=1}^{K}\left(P_{2 n}\left(U_{0}, V_{0}\right)\right)_{k}\right)\right\|_{H^{1} \times L^{2}} \\
+\left\|e^{C_{\eta} t} \sum_{k=K+1}^{\infty}\left(u_{0}, v_{0}\right)_{k}\right\|_{H^{1} \times L^{2}}+\left\|i\left(e^{C_{\eta n} t} \sum_{k=K+1}^{n}\left(P_{2 n}\left(u_{0}, v_{0}\right)\right)_{k}\right)\right\|_{H^{1} \times L^{2}}
\end{array}
$$

Pela continuidade, uniforme em $n$, das aplicações inclusão e projeção, temos:

$$
\begin{aligned}
\left\|i\left(e^{C_{\eta n} t} \sum_{k=K+1}^{n}\left(P_{2 n}\left(u_{0}, v_{0}\right)\right)_{k}\right)\right\|_{H^{1} \times L^{2}} & \leq M\left\|e^{C_{n n} t} \sum_{k=K+1}^{n}\left(P_{2 n}\left(u_{0}, v_{0}\right)\right)_{k}\right\|_{\mathbb{R}^{n} \times \mathbb{P}^{n}} \\
& =\left(\sum_{k=K+1}^{n}\left\|e^{C_{\eta n} t}\left(P_{2 n}\left(u_{0}, v_{0}\right)\right)_{k}\right\|_{\mathbb{R}^{n} \times 2^{n}}^{2}\right)^{1 / 2} \\
& \leq\left(\sum_{k=K+1}^{n}\left(e^{R e \lambda_{ \pm}^{n} t}\left\|\left(P_{2 n}\left(u_{0}, v_{0}\right)\right)_{k}\right\|_{\mathbb{E}^{n} \times 2^{n}}\right)^{2}\right)^{1 / 2} \\
& \left.\leq \epsilon t^{-\beta}\left(\sum_{k=K+1}^{n}\left\|\left(P_{2 n}\left(u_{0}, v_{0}\right)\right)_{k}\right\|_{\mathbb{R}^{n} \times \Xi^{n}}\right)^{2}\right)^{1 / 2} \\
& \leq \epsilon t^{-\beta}\left\|P_{2 n}\left(u_{0}, v_{0}\right)\right\|_{\mathbb{P}^{n} \times \mathbb{R}^{n}} \\
& \leq K \epsilon t^{-\beta}\left\|\left(u_{0}, v_{0}\right)\right\|_{H^{1} \times L^{2}}
\end{aligned}
$$

Analogamente. temos

$$
\left\|e^{C_{n} t} \sum_{k=K+1}^{\infty}\left(u_{0}, v_{0}\right)_{k}\right\|_{H^{1} \times L^{2}} \leq K \epsilon t^{-\beta}\left\|\left(u_{0}, v_{0}\right)_{k}\right\|_{H^{1} \times L^{2}}
$$


Consideramos um novo operador $B_{n}$ o qual possui os autoralores de $C_{\eta n}$ e os respectivos autovetores são os autovetores de $C_{\eta}$. Assim,temos:

$$
\begin{array}{r}
\left\|e^{C_{\eta} t} \sum_{k=1}^{K}\left(u_{0}, v_{0}\right)_{k}-i\left(e^{C_{\eta n} t} \sum_{k=1}^{K}\left(P_{2 n}\left(u_{0}, v_{0}\right)\right)_{k}\right)\right\|_{H^{1} \times L^{2}} \\
\leq\left\|e^{C_{\eta} t} \sum_{k=1}^{K}\left(u_{0}, v_{0}\right)_{k}-e^{B_{n} t} \sum_{k=1}^{K}\left(u_{0}, v_{0}\right)_{k}\right\|_{H^{1} \times L^{2}} \\
+\left\|e^{B_{n} t} \sum_{k=1}^{K}\left(u_{0}, v_{0}\right)_{k}-i\left(e^{C_{\eta n} t} \sum_{k=1}^{K}\left(P_{2 n}\left(u_{0}, v_{0}\right)\right)_{k}\right)\right\|_{H^{1} \times L^{2}}
\end{array}
$$

Assim, se cada $\lambda_{ \pm k}^{n}$, para $1 \leq k \leq K$, é real entāo

$$
\begin{array}{r}
\left\|\sum_{k=1}^{K} e^{C_{n} t}\left(u_{0}, v_{0}\right)_{k}-\sum_{k=1}^{K} e^{B_{n} t}\left(u_{0}, v_{0}\right)_{k}\right\|_{H^{1} \times L^{2}} \\
=\left\|\sum_{k=1}^{K} e^{\lambda_{+k} t}\left(u_{0}, v_{0}\right)_{+k}+e^{\lambda_{-k} t}\left(u_{0}, v_{0}\right)_{-k}-e^{\lambda_{+k}^{n} t}\left(u_{0}, v_{0}\right)_{+k}-e^{\lambda_{-k}^{n} t}\left(u_{0}, v_{0}\right)_{-k}\right\|_{H^{1} \times L^{2}} \\
\leq\left\|\sum_{k=1}^{K}\left(e^{\lambda_{+k} t}-e^{\lambda_{+k}^{n} t}\right)\left(u_{0}, v_{0}\right)_{+k}+\left(e^{\lambda_{-k} t}-e^{\lambda_{-k}^{n} t}\right)\left(u_{0}, v_{0}\right)_{-k}\right\|_{H^{1} \times L^{2}} \\
=\left(\left\|\sum_{k=1}^{K}\right\|\left(e^{\lambda_{+k} t}-e^{\lambda_{+k}^{n} t}\right)\left(u_{0}, v_{0}\right)_{+k}+\left(e^{\lambda_{-k} t}-e^{\lambda_{-k}^{n} t}\right)\left(u_{0}, v_{0}\right)_{-k} \|_{H^{1} \times L^{2}}^{2}\right)^{1 / 2} \\
\leq\left(\sum_{k=1}^{K}\left(2 \max \left\{\left|e^{\lambda_{+k} t}-e^{\lambda_{+k}^{n} t}\|\|\left(u_{0}, v_{0}\right)_{+k}\left\|\cdot\left|e^{\lambda_{-k} t}-e^{\lambda_{-k}^{n} t}\right|\right\|\left(u_{0} \cdot v_{0}\right)_{-k} \|\right\}\right)^{2}\right)^{1 / 2}\right. \\
\leq 2\left(\sum_{k=1}^{K}\left(\max _{1}\left\{\left|e^{\lambda_{+k} t}-e^{\lambda_{+k}^{n} t}\right|,\left|e^{\lambda_{-k} t}-e^{\lambda_{-k}^{n} t}\right|\right\} \cdot \max \left\{\left\|\left(u_{0}, v_{0}\right)_{-k}\right\|:\left\|\left(u_{0}, v_{0}\right)_{-k}\right\|\right\}\right)^{2}\right)^{1 / 2} \\
\leq 2 M\left(\left(\max _{1 \leq k \leq K}\left\{\left|e^{\lambda_{+k} t}-e^{\lambda_{+k}^{n} t}\right|,\left|e^{\lambda_{-k} t}-e^{\lambda_{-k}^{n} t}\right|\right\} \cdot\left(\sum_{k=1}^{K}\left\|\left(u_{0}, v_{0}\right)_{k}\right\|^{2}\right)^{1 / 2}\right.\right. \\
\leq 2 M t \max _{1 \leq k \leq K}\left\{\left|e^{\bar{\lambda}_{+k}^{n} t}\left\|\left|\lambda_{+k}-\lambda_{+k}^{n}\right|,\left|e^{\bar{\lambda}_{-k}^{n} t} \| \lambda \lambda_{-k}-\lambda_{-k}^{n}\right|\right\}\left\|\left(u_{0}, v_{0}\right)\right\|_{H^{1} \times L^{2}}\right.\right. \\
\leq \epsilon t^{-\beta}\left\|\left(u_{0}, v_{0}\right)\right\|_{H^{1} \times L^{2}}
\end{array}
$$

para $n \geq n_{1} \geq n_{0}$, onde $\bar{\lambda}_{-k}^{n}$ está entre $\lambda_{-k}$ e $\lambda_{-k}^{n}$. $\tilde{\lambda}_{+k}^{n}$ está entre $\lambda_{+k}$ e $\lambda_{+k}^{n}$.

No caso $\lambda_{ \pm k}$ complexo, se denotamos por $\left(u_{0}, v_{0}\right)_{k}$ a componente de $\left(u_{0}, v_{0}\right)$ em $E_{k}$ então $\left(u_{0}, v_{0}\right)_{k}=2 a(\cos \delta,-\operatorname{sen} \delta)$ na base $\psi_{+k}, \psi_{-k}$. Neste caso.

$$
e^{C_{\eta} t}\left(u_{0}, v_{0}\right)_{k}=2 a e^{\alpha_{k} t}\left(\cos \left(\beta_{k} t+\delta\right) u_{+k}-\operatorname{sen}\left(3_{k} t+\delta\right) \tau_{-k}\right)
$$

e

$$
e^{B_{n} t}\left(u_{0}, v_{0}\right)_{k}=2 a e^{\alpha_{k}^{n} t}\left(\cos \left(\beta_{k}^{n} t+\delta\right) \dot{v}_{+k}-\operatorname{sen}\left(3_{k}^{n} t+\delta\right) u_{-k}\right)
$$


, onde $\lambda_{ \pm k}=\alpha_{k} \pm \beta_{k}$ e $\lambda_{ \pm k}^{n}=\alpha_{k}^{n} \pm \beta_{k}^{n}$, então

$$
\begin{array}{r}
\left\|e^{C_{\eta} t}\left(u_{0}, v_{0}\right)_{k}-e^{B_{n} t}\left(u_{0}, v_{0}\right)_{k}\right\|_{H^{1} \times L^{2}} \\
\leq\left\|2 a e^{\alpha_{k} t}\left[\left(\cos \left(\beta_{k} t+\delta\right)-\cos \left(\beta_{k}^{n} t+\delta\right)\right) \psi_{+k}-\left(\operatorname{sen}\left(\beta_{k} t+\delta\right)-\operatorname{sen}\left(\beta_{k}^{n} t+\delta\right)\right) \psi_{-k}\right]\right\| \\
+\left\|2 a\left[\cos \left(\beta_{k}^{n} t+\delta\right) \psi_{+k}-\operatorname{sen}\left(\beta_{k}^{n} t+\delta\right) \psi_{-k}\right]\right\|\left|e^{\alpha_{+k} t}-e^{\alpha_{+k}^{n} t}\right| \\
\leq e^{\alpha_{+k} t} t\left|\beta_{k}-\beta_{k}^{n}\right|\left\|-2 a \operatorname{sen}\left(\bar{\beta}_{k}^{n}+\delta\right) \psi_{+k}-2 a \cos \left(\bar{\beta}_{k}^{n} t+\delta\right) \psi_{-k}\right\| \\
+e^{\bar{\alpha}_{+k} t} t\left|\alpha_{k}-\alpha_{k}^{n}\right|\left\|2 a \cos \left(\beta_{k}^{n} t+\delta\right) \psi_{+k}-2 a \operatorname{sen}\left(\beta_{k}^{n} t+\delta\right) \psi_{-k}\right\| \\
\leq L\left(e^{\alpha_{+k} t} t\left|\beta_{k}-\beta_{k}^{n}\right|+e^{\bar{\alpha}_{+k} t} t\left|\alpha_{k}-\alpha_{k}^{n}\right|\right)\left\|\left(u_{0}, v_{0}\right)_{k}\right\|
\end{array}
$$

Se $\left(P_{2 n}\left(u_{0}, v_{0}\right)\right)_{k}=a_{+k}^{n} \psi_{+k}^{n}+a_{-k}^{n} \psi_{-k}^{n}$ e $\left(u_{0}, v_{0}\right)_{k}=a_{+k} \psi_{+k}+a_{-k} \psi_{-k}$ então

$$
\begin{array}{r}
\left\|e^{B_{n} t} \sum_{k=1}^{K}\left(u_{0}, v_{0}\right)_{k}-i\left(e^{C_{n n} t} \sum_{k=1}^{K}\left(P_{2 n}\left(u_{0}, v_{0}\right)\right)_{k}\right)\right\|_{H^{1} \times L^{2}} \\
\leq\left\|\sum_{k=1}^{K} e^{B_{n} t}\left(u_{0}, v_{0}\right)_{k}-\sum_{k=1}^{K} e^{B_{n} t}\left(a_{+k}^{n} v_{+k}+a_{-k}^{n} \psi_{-k}\right)\right\|_{H^{1} \times L^{2}} \\
+\left\|\sum_{k=1}^{K} e^{B_{n} t}\left(a_{+k}^{n} \psi_{+k}+a_{-k}^{n} \psi_{-k}\right)-\sum_{k=1}^{K} i\left(e^{C_{\eta n} t}\left(P_{2 n}\left(u_{0}, v_{0}\right)\right)_{k}\right)\right\|_{H^{1} \times L^{2}} \\
\leq e^{-\alpha_{1}^{n} t} \sum_{k=1}^{K}\left(\left|a_{+k}-a_{+k}^{n}\right|\left\|\psi_{+k}\right\|+\left|a_{-k}-a_{-k}^{n}\right|\left\|\psi_{-k}\right\|\right) \\
+\left\|\sum_{k=1}^{K} e^{B_{n} t}\left(a_{+k}^{n} \psi_{+k}+a_{-k}^{n} \psi_{-k}\right)-\sum_{k=1}^{K} i\left(e^{C_{\eta n} t} a_{+k}^{n} v_{+k}^{n}+a_{-k}^{n} \psi_{-k}^{n}\right)\right\|_{H^{1} \times L^{2}}
\end{array}
$$

Assim precisamos estimar $\left|a_{+k}-a_{+k}^{n}\right|$ e $\left|a_{-k}-a_{-k}^{n}\right|$. Como $a_{+k}^{n}=\frac{b_{+k}^{n}}{c_{+k}^{n}}$ e $a_{+k}=\frac{b_{+k}}{c_{+k}}$ onde

$$
\begin{gathered}
b_{+k}^{n}=\left\langle P_{2 n}\left(u_{0}, v_{0}\right), \psi_{+k}^{n}\right)\left\|\psi_{-k}^{n}\right\|^{2}-\left\langle P_{2 n}\left(u_{0}, v_{0}\right), 2_{-k}^{n}\right\rangle\left\langle\psi_{-k}^{n}, \psi_{+k}^{n}\right\rangle, \\
c_{+k}^{n}=\left\|\psi_{+k}^{n}\right\|^{2}\left\|\psi_{-k}^{n}\right\|^{2}-\left\langle\psi_{+k}^{n} \cdot \psi_{-k}^{n}\right\rangle^{2}
\end{gathered}
$$

e

$$
\begin{gathered}
b_{+k}=\left\langle\left(u_{0}, v_{0}\right), \psi_{+k}\right)\left\|\psi_{-k}\right\|^{2}-\left(\left(u_{0}, v_{0}\right): \psi_{-k}\right\rangle\left\langle\psi_{-k}, \psi_{+k}\right\rangle, \\
c_{+k}=\left\|\psi_{+k}\right\|^{2}\left\|\psi_{-k}\right\|^{2}-\left\langle\dot{\psi}_{\perp_{k}} \cdot \psi_{-k}\right\rangle^{2} .
\end{gathered}
$$

Assim,

$$
\left|a_{-k}-a_{-k}^{n}\right| \leq \frac{\left|c_{+k}^{n}\right|\left|b_{+k}-b_{+k}^{n}\right|+\left|c_{+k}-c_{+k}^{n}\right|\left|b_{+k}^{n}\right|}{\left|c_{+k}\right|\left|c_{+k}^{n}\right|}
$$

e basta estimarmos $\left|b_{+k}-b_{+k}^{n}\right|$ e $\left|c_{+k}-c_{+k}^{n}\right|$. Neste caso faremos duas distinções:

I) $\left(u_{0}, v_{0}\right)=i\left(U_{0}, V_{0}^{r}\right)$ e $P_{2 n}\left(u_{0}, v_{0}\right)=\left(U_{0}, V_{0}\right)$ para $\left(U_{0} . V_{0}\right) \in \mathcal{A}_{\eta n}$ : 
II) $\left(u_{0}, v_{0}\right)$ em $C^{1+\alpha} \times C^{\alpha}$.

Como:

i) $\left\|\psi_{+k}\right\|_{H^{1} \times L^{2}}=\left\|\psi_{+k}^{n}\right\|_{\mathbb{R}^{n} \times \mathbb{R}^{n}}+O\left(\frac{1}{n}\right)$,

ii) $\left\langle\psi_{+k}, \psi_{-k}\right\rangle_{H^{1} \times L^{2}}=\left\langle\psi_{+k}^{n}, \psi_{-k}^{n}\right)_{\mathbb{R}^{n} \times \mathbb{R}^{n}}+O\left(\frac{1}{n}\right)$

iii) $\left\langle i\left(P_{n}\left(u_{0}\right)\right) \cdot \cos (k \pi x)\right\rangle_{H^{1}}=\sum_{i=1}^{n-1} \int_{x_{i}}^{x_{i+1}} n\left(u_{i+1}-u_{i}\right) k \pi \operatorname{sen}(k \pi x) d x$,

iv) $\left\langle P_{n}\left(u_{0}\right), P_{n}(\cos (k \pi x)\rangle_{H_{d}^{1}}=\sum_{i=1}^{n-1} \int_{x_{i}}^{x_{i+1}} n\left(u_{i+1}-u_{i}\right) k \pi \operatorname{sen}\left(k \pi \overline{x_{i}}\right) d x\right.$.

Logo:

$$
\mid\left\langle i\left(P_{n}\left(u_{0}\right)\right), \cos (k \pi x)\right\rangle_{H^{1}}-\left(P_{n}\left(u_{0}\right), P_{n}(\cos (k \pi x))_{H_{d}^{1}} \mid \leq k^{2} \pi^{2} \sum_{i=1}^{n-1} \frac{1}{n}\left(u_{i+1}-u_{i}\right) .\right.
$$

Agora, relembrando que se $\left(u_{0}, v_{0}\right)=i\left(U_{0}, V_{0}\right)$ para algum $\left(U_{0} \cdot V_{0}\right) \in \mathcal{A}_{\eta n}$ então, como já vimos no capítulo 4 , Teorema $4.2, \bigcup_{n} \mathcal{A}_{\eta n}$ é limitado em $H_{d}^{2} \times H_{d}^{1}$ e portanto $n\left|u_{i+1}-u_{i}\right| \leq$ $\|U\|_{H_{d}^{1}}+\left\|C^{-}\right\|_{H_{d}^{2}} \leq 2 K$ para $1 \leq i \leq n-1$ e assim,

$$
\mid\left\langle i\left(P_{n}\left(u_{0}\right)\right) \cdot \cos (k \pi x)\right\rangle_{H^{1}}-\left\langle P_{n}\left(u_{0}\right), P_{n}(\cos (k \pi x)\rangle_{H_{d}}\right| \leq \frac{k^{2} \pi^{2}}{n}\left(\left\|U_{0}\right\|_{H_{d}^{1}}+\left\|U_{0}\right\|_{H_{d}^{2}}\right) .
$$

Também:

v) $\left(i\left(P_{n}\left(v_{0}\right)\right) \cdot \cos (k \pi x)\right\rangle_{L^{2}}=\sum_{i=1}^{n} \int_{\frac{i-1}{n}}^{\frac{i}{n}} v_{i} \cos (k \pi x) d x$, iv) $\left(P_{n}\left(v_{0}\right) \cdot P_{n}(\cos (k \pi x)\rangle_{L_{d}^{2}}=\sum_{i=1}^{n} \int_{\frac{i=1}{n}}^{\frac{1}{n}} v_{i} \cos \left(k \pi x_{i}\right) d x\right.$.

Logo:

$$
\mid\left\langle i\left(P_{n}\left(v_{0}\right)\right): \cos (k \pi x)\right\rangle_{L^{2}}-\left\langle P_{n}\left(v_{0}\right),\left.P_{n}(\cos (k \pi x))\right|_{L_{d}^{2}} \leq k \pi \sum_{i=1}^{n} \frac{1}{n^{2}} v_{i} .\right.
$$

Agora, relembrando que se $\left(u_{0}, v_{0}\right)=i\left(U_{0}, V_{0}\right)$ para algum $\left(U_{0}, V_{0}\right) \in \mathcal{A}_{\eta n}$ então, utilizando novamente que $\bigcup_{n} \mathcal{A}_{\eta n}$ é limitado em $H_{d}^{2} \times H_{d}^{1}$ e portanto $\left|v_{i}\right| \leq\|V\|_{L_{d}^{2}}+\|V\|_{H_{d}^{1}} \leq 2 K$ para $1 \leq i \leq n$ e assim.

$$
\mid\left\langle i\left(P_{n}\left(v_{0}\right)\right), \cos (k \pi x)\right\rangle_{L^{2}}-\left(P_{n}\left(v_{0}\right), P_{n}(\cos (k \pi x)\rangle_{L_{d}^{2}} \mid \leq \frac{k^{2} \pi^{2}}{n}\left(\left\|V_{0}\right\|_{L_{d}^{2}}+\left\|V_{0}\right\|_{H_{d}^{1}}\right) .\right.
$$

Logo,

$$
\left|\left\langle i\left(P_{2 n}\left(u_{0}, v_{0}\right)\right), \iota_{ \pm k}\right\rangle_{H^{1} \times L^{2}}-\left\langle P_{2 n}\left(u_{0}, v_{0}\right), \psi_{ \pm k}^{n}\right\rangle_{H_{d}^{1} \times L_{d}^{2}}\right| \leq \frac{\overline{K^{n}}}{n}\left\|\left(U_{0}, V_{0}\right)\right\|_{H_{d}^{2} \times H_{d}^{1}} .
$$

Se $\left(u_{0} . v_{0}\right) \neq i\left(U_{0} . I_{0}\right)$, mas $\left(u_{0}, v_{0}\right) \in C^{1+\alpha} \times C^{\alpha}$ então nós temos $\left|u_{i+1}-u_{i}\right| \leq$ $n^{-1}\left\|u_{0}\right\|_{C^{1+\alpha}} \cdot\left|u_{i}\right| \leq\|v\|_{C^{\circ}}$ e assim

$$
\begin{array}{r}
\mid\left\langle u_{0}, \cos (k \pi x)\right\rangle_{H^{1}}-\left\langle P_{n}\left(u_{0}\right), P_{n}(\cos (k \pi x)\rangle_{H_{d}^{1}}\right| \\
\leq\left|\left\langle u_{0}, \cos (k \pi x)\right\rangle_{H^{1}}-\left\langle i\left(P_{n}\left(u_{0}\right)\right) \cdot \cos (k \pi x)\right\rangle_{H_{d}^{1}}\right| \\
+\mid\left\langle i\left(P_{n}\left(u_{0}\right)\right), \cos (k \pi x)\right\rangle_{H^{1}}-\left\langle P_{n}\left(u_{0}\right) \cdot P_{n}(\cos (k \pi x)\rangle_{H_{d}^{1}}\right| .
\end{array}
$$


Mas,

$$
\left\|\left(u_{0}, v_{0}\right)-i\left(P_{2 n}\left(u_{0}, v_{0}\right)\right)\right\|_{H^{1} \times L^{2}} \leq \frac{1}{n^{\alpha}}\left\|\left(u_{0}, v_{0}\right)\right\|_{C^{1+\alpha} \times C^{\alpha}}
$$

E portanto,

$$
\left|\left\langle\left(u_{0}, v_{0}\right), \psi_{ \pm k}\right\rangle_{H^{\mathrm{x}} \times L^{2}}-\left\langle P_{2 n}\left(u_{0}, v_{0}\right), \psi_{ \pm k}^{n}\right\rangle_{H_{d}^{1} \times L_{d}^{2}}\right| \leq \frac{\tilde{K}}{n^{\alpha}}\left\|\left(u_{0}, v_{0}\right)\right\|_{C^{1+\alpha} \times C^{\alpha}}
$$

Logo, para o caso I)

$$
\left|b_{+k}-b_{+k}^{n}\right| \leq M n^{-1}\left\|\left(U_{0}, V_{0}\right)\right\|_{H_{d}^{2} \times H_{d}^{1}}
$$

para o caso II),

$$
\left|b_{+k}-b_{+k}^{n}\right| \leq M n^{-\alpha}\left\|\left(u_{0}, v_{0}\right)\right\|_{C^{1+\alpha} \times C^{\alpha}}
$$

e de forma análoga, para todo $k$ tal que $1 \leq k \leq K$ temos

$$
\left|c_{+k}-c_{+k}^{n}\right| \leq M n^{-1}
$$

Analogamente, calculamos $\left|a_{-k}-a_{-k}^{n}\right|$.

Voltando a estimativa $e^{-\alpha_{1}^{n} t} \sum_{k=1}^{K}\left(\left|a_{+k}-a_{+k}^{n} !\left\|\psi_{+k}\right\|+\right| a_{-k}-a_{-k}^{n}\left|\| \psi_{-k}\right| \mid\right)$, temos, no caso I)

$$
\begin{array}{r}
e^{-\alpha_{1}^{n} t} \sum_{k=1}^{K}\left(\left|a_{+k}-a_{+k}^{n}\right|\left\|\psi_{+k}\right\|+\left|a_{-k}-a_{-k}^{n}\right|\left\|\psi_{-k}\right\|\right) \\
\leq e^{-\alpha_{1}^{n} t} \tilde{M} n^{-1}\left\|\left(U_{0}, V_{0}\right)\right\|_{H_{d}^{2} \times H_{d}} \sum_{k=1}^{K}\left(\left\|\psi_{+k}\right\|+\left\|\psi_{-k}\right\|\right) \\
\leq \epsilon t^{-\beta}\left\|\left(U_{0}, V_{0}\right)\right\|_{H_{d}^{2} \times H_{d}^{1}}
\end{array}
$$

e para o caso II)

$$
\begin{array}{r}
e^{-\alpha_{1}^{n} t} \sum_{k=1}^{K}\left(\left|a_{+k}-a_{+k}^{n}\right|\left\|\psi_{+k}\right\|+\mid a_{-k}-a_{-k}^{n}\|\| \psi_{-k} \|\right) \\
\leq e^{-\alpha_{k}^{n} t} \tilde{M} n^{-\alpha}\left\|\left(u_{0}, v_{0}\right)\right\|_{C^{1+\alpha} \times C^{\alpha}} \sum_{k=1}^{K}\left(\left\|\psi_{+k}\right\|+\left\|\psi_{-k}\right\|\right) \\
\leq \epsilon t^{-\beta}\left\|\left(u_{0}, v_{0}\right)\right\|_{C^{1+\alpha} \times C^{\alpha}}
\end{array}
$$

Agora vamos estimar

$$
\left\|\sum_{k=1}^{K} e^{B_{n} t}\left(a_{+k}^{n} \psi_{+k}+a_{-k}^{n} \psi_{-k}\right)-\sum_{k=1}^{K} i\left(e^{C_{n n} t} a_{+k}^{n} \psi_{+k}^{n}+a_{-k}^{n} \psi_{-k}^{n}\right)\right\|_{H^{1} \times L^{2}}
$$


Para isso. ramos considerar a inclusão complexa, como inclusão da parte real e inclusão da parte imginária. Neste caso estaremos considerando as soluções complexas.

Como $a_{+k}^{n} v_{-k}+a_{-k}^{n} \psi_{-k}=b_{+k}^{n} \phi+k+b_{-k}^{n} \phi-k$ e $a_{+k}^{n} \psi_{+k}^{n}+a_{-k}^{n} \psi_{-k}^{n}=b_{+k}^{n} \phi+k^{n}+b_{-k}^{n} \phi-k^{n}$ onde $b_{+k}=1 / 2\left(a_{+k}^{n}+a_{-k}^{n}\right)$ e $b_{-k}=1 / 2\left(a_{+k}^{n}-a_{-k}^{n}\right)$ então

$$
e^{B_{n} \iota}\left(a_{+k}^{n} \psi_{+k}+a_{-k}^{n} \psi_{-k}\right)=e^{\lambda_{+k}^{n} t} b_{+k}^{n} \phi_{+k}+e^{\lambda_{-k}^{n} t} b_{-k}^{n} \phi_{-k}
$$

$\mathrm{e}$

$$
\begin{aligned}
i\left(e^{C_{\eta n} t}\left(a_{+k}^{n} \psi_{+k}^{n}+a_{-k}^{n} \psi_{-k}^{n}\right)\right) & =i\left(e^{\lambda_{+k}^{n} t} b_{+k}^{n} \phi_{+k}^{n}+e^{\lambda_{-k}^{n} t} b_{-k}^{n} \phi_{-k}^{n}\right) \\
= & b_{+k}^{n} i\left(e^{\lambda_{+k}^{n} t} \phi_{+k}^{n}\right)+b_{-k}^{n} i\left(e^{\lambda_{-k}^{n} t} \phi_{-k}^{n}\right)
\end{aligned}
$$

Logo:

$$
\begin{array}{r}
\left\|e^{B_{n} t}\left(a_{+k}^{n} \psi_{+k}+a_{-k}^{n} \psi_{-k}\right)-i\left(e^{C_{\eta n} t}\left(a_{+k}^{n} \psi_{+k}^{n}+a_{-k}^{n} \psi_{-k}^{n}\right)\right)\right\| \\
\leq\left|b_{+k}^{n}\right|\left\|e^{\lambda_{+k}^{n} t} \phi_{+k}-i\left(e^{\lambda_{+k}^{n} t} \phi_{+k}^{n}\right)\right\|+\left|b_{-k}^{n}\right|\left\|e^{\lambda_{-k}^{n} t} \phi_{-k}-i\left(e^{\lambda_{-k}^{n} t} \phi_{-k}^{n}\right)\right\| \\
=\left|b_{+k}^{n}\right|\left\|e^{\lambda_{+k}^{n} t} \phi_{+k}-e^{\lambda_{+k}^{n} t} i\left(\phi_{+k}^{n}\right)\right\|+\left|b_{-k}^{n}\right|\left\|e^{\lambda_{-k}^{n} t} \phi_{-k}-e^{\lambda_{-k}^{n} t} i\left(\phi_{-k}^{n}\right)\right\| \\
\leq\left|b_{+k}^{n}\right| e^{\alpha_{+k}^{n} t}\left\|\phi_{+k}-i\left(\phi_{+k}^{n}\right)\right\|+\left|b_{-k}^{n}\right| e^{\alpha_{-k}^{n} t}\left\|\phi_{-k}-i\left(\phi_{-k}^{n}\right)\right\| \\
\leq\left|b_{+k}^{n}\right| e^{\alpha_{+k}^{n} t} K / n\left\|\dot{\phi}_{+k}\right\|+\left|b_{-k}^{n}\right| e^{\alpha_{-k}^{n} t} K / n\left\|\phi_{-k}\right\| \\
\leq e^{\alpha_{+k}^{n} t} \tilde{K} / n\left\|\left(u_{0}, v_{0}\right)_{k}\right\| \leq \epsilon t^{-\beta}\left\|\left(u_{0}, v_{0}\right)_{k}\right\|
\end{array}
$$

Portanto.

$\left\|\sum_{k=1}^{K} e^{B_{n} t}\left(a_{+k}^{n} v_{+k}+a_{-k}^{n} \psi_{-k}\right)-\sum_{k=1}^{K} i\left(e^{C_{\eta n} t} a_{+k}^{n} \psi_{+k}^{n}+a_{-k}^{n} \psi_{-k}^{n}\right)\right\|_{H^{1} \times L^{2}} \leq \epsilon t^{-\beta}\left\|\left(u_{0}, v_{0}\right)\right\|_{H^{1} \times L^{2}}$

Finalmente. para o caso I),

$$
\left\|e^{B_{n} t} \sum_{k=1}^{K}\left(u_{0} \cdot v_{0}\right)_{k}-i\left(e^{C_{\eta n} t} \sum_{k=1}^{K}\left(P_{2 n}\left(u_{0}, v_{0}\right)\right)_{k}\right)\right\|_{H^{1} \times L^{2}} \leq \epsilon t^{-B}\left\|\left(u_{0}, v_{0}\right)\right\|_{H^{1} \times L^{2}}
$$

para o caso II)

$$
\left\|e^{B_{n} t} \sum_{k=1}^{K}\left(u_{0}, v_{0}\right)_{k}-i\left(e^{C_{\eta n} t} \sum_{k=1}^{K}\left(P_{2 n}\left(u_{0}, v_{0}\right)\right)_{k}\right)\right\|_{H^{1} \times L^{2}} \leq \epsilon t^{-\beta}\left\|\left(u_{0}, v_{0}\right)\right\|_{C^{1+\alpha} \times C^{\alpha}} .
$$




\subsection{Comparação dos semigrupos não lineares}

Sejam $T_{\eta}(t)$ e $T_{\eta n}(t)$ os semigrupos não lineares associados a (1.17) e (1.27), respectivamente. Pela fórmula da variação das constantes e para $\left(U_{0}, V_{0}\right) \in \mathcal{A}_{\eta n}$ temos:

$$
\begin{aligned}
& T_{\eta n}\left(t,\left(U_{0}, V_{0}\right)\right)=e^{C_{\eta n} t}\left(U_{0}, V_{0}\right)+\int_{0}^{t} e^{C_{\eta n}(t-s)} H\left(T_{\eta n}\left(s,\left(U_{0}, V_{0}\right)\right)\right) d s \\
& T_{\eta}\left(t, i\left(U_{0}, V_{0}\right)\right)=e^{C_{\eta} t} i\left(U_{0}, V_{0}\right)+\int_{0}^{t} e^{C_{\eta}(t-s)} h\left(T_{\eta}\left(s, i\left(U_{0}, V_{0}\right)\right)\right) d s
\end{aligned}
$$

Então, para $t \in(0, \tau)$

$$
\begin{array}{r}
\left\|T_{\eta}\left(t, i\left(U_{0}, V_{0}\right)\right)-i\left(T_{\eta n}\left(t,\left(U_{0}, V_{0}\right)\right)\right)\right\|_{H^{1} \times L^{2}} \\
\leq\left\|e^{C_{\eta} t} i\left(U_{0}, V_{0}\right)-i\left(e^{C_{\eta n} t}\left(U_{0}, V_{0}\right)\right)\right\|_{H^{1} \times L^{2}} \\
+\left\|\int_{0}^{t} e^{C_{\eta}(t-s)} h\left(T_{\eta}\left(s, i\left(U_{0}, V_{0}\right)\right)\right)-i\left(e^{C_{\eta n}(t-s)} P_{2 n} h\left(T_{\eta}\left(s . i\left(U_{0}, V_{0}\right)\right)\right)\right) d s\right\|_{H^{1} \times L^{2}} \\
+\left\|\int_{0}^{t} i\left(e^{C_{\eta n}(t-s)} P_{2 n} h\left(T_{\eta}\left(s, i\left(U_{0}, V_{0}\right)\right)\right)\right)-i\left(e^{C_{\eta n}(t-s)} H\left(T_{\eta n}\left(s,\left(U_{0}, V_{0}\right)\right)\right)\right) d s\right\|_{H^{1} \times L^{2}} \\
\leq \epsilon t^{-\beta}\left\|\left(U_{0}, V_{0}\right)\right\|_{\mathbb{R}^{n} \times \mathbb{R}^{n}} \\
+\int_{0}^{t}\left\|e^{C_{\eta}(t-s)} h\left(T_{\eta}\left(s, i\left(U_{0}, V_{0}\right)\right)\right)-i\left(e^{C_{\eta n}(t-s)} P_{2 n} h\left(T_{\eta}\left(s . i\left(U_{0}, V_{0}\right)\right)\right)\right)\right\|_{H^{1} \times L^{2}} d s \\
+\int_{0}^{t}\left\|i\left(e^{C_{\eta n}(t-s)} P_{2 n} h\left(T_{\eta}\left(s, i\left(U_{0}, V_{0}\right)\right)\right)\right)-i\left(e^{C_{\eta n}(t-s)} H\left(T_{\eta n}\left(s .\left(U_{0}, V_{0}\right)\right)\right)\right)\right\|_{H^{1} \times L^{2} d s}
\end{array}
$$

Como $H\left(T_{\eta n}\left(s,\left(U_{0}, V_{0}\right)\right)\right)=P_{2 n}\left(h\left(i\left(T_{\eta n}\left(s,\left(U_{0} \cdot V_{0}\right)\right)\right)\right)\right)$ então

$$
\begin{array}{r}
\epsilon t^{-\beta}\left\|\left(U_{0}, V_{0}\right)\right\|_{\mathbb{R}^{n} \times \mathbb{R}^{n}} \\
+\int_{0}^{t}\left\|e^{C_{\eta}(t-s)} h\left(T_{\eta}\left(s, i\left(U_{0}, V_{0}\right)\right)\right)-i\left(e^{C_{\eta n}(t-s)} P_{2 n} h\left(T_{\eta}\left(. s . i\left(U_{0}, V_{0}\right)\right)\right)\right)\right\|_{H^{1} \times L^{2}} d s \\
+\int_{0}^{t}\left\|i\left(e^{C_{\eta n}(t-s)} P_{2 n} h\left(T_{\eta}\left(s, i\left(U_{0}, V_{0}\right)\right)\right)\right)-i\left(e^{C_{\eta n}(t-s)} H\left(T_{\eta n}\left(s .\left(U_{0}, V_{0}\right)\right)\right)\right)\right\|_{H^{1} \times L^{2}} d s \\
\leq \epsilon t^{-\beta}\left\|\left(U_{0}, V_{0}\right)\right\|_{H_{d}^{2} \times H_{d}^{1}}+\epsilon \int_{0}^{t}(t-s)^{-\beta}\left\|h\left(T_{\eta}\left(s . i\left(U_{0}, V_{0}\right)\right)\right)\right\|_{C^{1+\alpha} \times C^{\alpha}} d s \\
+\int_{0}^{t}\left\|e^{C_{\eta n}(t-s)}\right\|\left\|P_{2 n}\left(h\left(T_{\eta}\left(s, i\left(U_{0}, V_{0}\right)\right)\right)\right)-P_{2 n}\left(h\left(i\left(T_{\eta n}\left(s .\left(U_{0}, V_{0}^{r}\right)\right)\right)\right)\right)\right\|_{H_{d}^{1} \times L_{d}^{2} d s}
\end{array}
$$

Também temos que $i\left(U_{0}, V_{0}\right)$ é limitado em $H^{1} \times L^{2}$. logo $\left(T_{\eta}\left(s, i\left(U_{0}, V_{0}\right)\right)\right)_{1}$ é limitada em $C^{\alpha}$ e portanto $\left\|h\left(T_{\eta}\left(s, i\left(U_{0}, V_{0}\right)\right)\right)\right\|_{C^{i+\alpha} \times C^{\alpha}}$ é limitado para todo $\left(U_{0}, V_{0}\right) \in \bigcup_{n} \mathcal{A}_{\eta n}$. 
Assim,

$$
\begin{array}{r}
\epsilon t^{-\beta}\left\|\left(U_{0}, V_{0}\right)\right\|_{H_{d}^{2} \times H_{d}^{1}}+\epsilon \int_{0}^{t}(t-s)^{-\beta}\left\|h\left(T_{\eta}\left(s, i\left(U_{0}, V_{0}\right)\right)\right)\right\|_{C^{1+\alpha} \times C^{a}} d s \\
+\int_{0}^{t}\left\|e^{C_{\eta n}(t-s)}\right\|\left\|P_{2 n}\left(h\left(T_{\eta}\left(s, i\left(U_{0}, V_{0}\right)\right)\right)\right)-P_{2 n}\left(h\left(i\left(T_{\eta n}\left(s,\left(U_{0}, V_{0}\right)\right)\right)\right)\right)\right\|_{H_{d}^{1} \times L_{d}^{2}} d s \\
\leq \epsilon t^{-\beta} K_{0}+\epsilon \tau \frac{t^{-\beta} K_{0}}{1-\beta} \\
+L \int_{0}^{t}\left\|\left(T_{\eta}\left(s, i\left(U_{0}, V_{0}\right)\right)\right)-i\left(T_{\eta n}\left(s,\left(U_{0}, V_{0}\right)\right)\right)\right\|_{H^{1} \times L^{2}} d s
\end{array}
$$

Portanto, pela Desigualdade de Gronwall, temos que existe uma constante $M(3, \tau, L)$ tal que

$$
\left\|T_{\eta}\left(t, i\left(U_{0}, V_{0}\right)\right)-i\left(T_{\eta n}\left(t,\left(U_{0}, I_{0}^{r}\right)\right)\right)\right\|_{H^{1} \times L^{2}} \leq M \epsilon K_{0} t^{-\beta},
$$

para $t \in(0 . \tau),\left(U_{0}, V_{0}\right) \in \mathcal{A}_{\eta n}$ e para $n \leq n(\epsilon)$

\subsection{Semi-continuidade superior dos atratores $\mathcal{A}_{\eta} \mathrm{e}$} $i\left(\mathcal{A}_{\eta n}\right)$ em $H^{1} \times L^{2}$

Como $\bigcup_{n} \mathcal{A}_{\eta n}$ é limitado em $\mathbb{R}^{n} \times \mathbb{R}^{n}$ e também $\|i(U, V)\|_{H^{1} \times L^{2}} \leq\|(U, V)\|_{\mathbb{E}^{n} \times \mathbb{R}^{n}}$ então $\left\|i\left(\bigcup_{n} \mathcal{A}_{\eta n}\right)\right\|_{H^{1} \times L^{2}} \leq\left\|\bigcup_{n} \mathcal{A}_{n}\right\|_{\mathbb{R}^{n} \times \mathbb{R}^{n}} \leq K$.

$\mathrm{O}$ atrator $\mathcal{A}_{\eta}$ atrai limitados de $H^{1} \times L^{2}$, ou seja, $\forall \delta>0$, existe $\tau=\tau(\delta)$ tal que

$$
\operatorname{dist}\left(T_{\eta}\left(\tau, i\left(\phi_{n}\right)\right) \cdot \mathcal{A}_{\eta}\right) \leq \delta / 2
$$

para toda $\dot{\phi}_{n} \in \mathcal{A}_{\eta n}$ e para todo $n$.

Os atratores $\mathcal{A}_{\eta n}$ são invariantes, assim se $\imath_{n} \in \mathcal{A}_{\eta n}$ então existe $\phi_{n} \in \mathcal{A}_{\eta n}$ tal que $T_{\eta n}\left(\tau, \phi_{n}\right)=\psi_{n}$.

Assim, escolhemos $n_{0}(\delta)=n(\epsilon(\delta))>0$ tal que

$$
\left\|T_{\eta}\left(\tau . i\left(\phi_{n}\right)\right)-i\left(T_{\eta n}\left(\tau, \phi_{n}\right)\right)\right\| \leq M \epsilon \tau^{-\beta}\left\|\phi_{n}\right\| \leq \delta / 2
$$

para $n \geq n_{0}(\delta)$

Assim,

$$
\operatorname{dist}\left(i\left(\psi_{n}\right), \mathcal{A}_{\eta}\right) \leq \operatorname{dist}\left(i\left(\psi_{n}\right), T_{\eta}\left(\tau, i\left(o_{n}\right)\right)+\operatorname{dist}\left(T_{\eta}\left(\tau, i\left(\varphi_{n}\right)\right), \mathcal{A}_{\eta}\right) \leq \delta\right.
$$

para $\imath_{n} \in \mathcal{A}_{\eta n}$, para todo $n \geq n_{0}(\delta)$. 



\section{Apêndice A}

\section{Convergência em $n$ e $\eta$ dos}

\section{autovalores do problema linearizado}

Este apêndice esta subdivido em três seções. Na primeira delas obtemos algumas estimativas que serão necessárias para o estudo da convergência dos autovalores e autovetores. Na segunda seção estudamos a relação entre o espectro de um operador diferencial de segunda ordem e o espectro da sua discretização. Na terceira seção, estudamos a convergência em $n$ e $\eta$ dos autovalores da discretizaçāo do problema de ondas linearizado para os autoralores do problema contínuo.

\section{A.1 Algumas estimativas}

Nesta seção utilizaremos a notação do Capítulo $\bar{j}$, ou seja, as aplicações $i: \mathbb{R}^{n} \rightarrow H^{1}(0,1)$ e $P_{n}: H^{1}(0,1) \rightarrow \mathbb{R}^{n}$ são dadas por

$$
i(U)=u(x)=u_{1} \chi_{\left[0, \frac{1}{2 n}\right)}+\sum_{i=1}^{n_{1}}\left(u_{i}+\left(u_{i+1}-u_{i}\right) n\left(x-x_{i}\right)\right) \chi_{\left[\frac{2 i-1}{2 n}, \frac{2 i+1}{2 n}\right)}+u_{n} \chi_{\left[\frac{2 n-1}{2 n}, 1\right]}
$$

e $P_{n}(u(x))=\left(u_{1}, \ldots, u_{n}\right)$ onde $u_{2}=u\left(x_{i}\right)$ para $x_{i}=\frac{2 i-1}{2 n}$.

Para estudar a convergência de um número fixo de autovalores é necessário algumas estimativas sobre certas expressões e seus correspondentes discretos, através da projeção. De forma análoga, fazemos estimativas sobre certas expressões e seus correspondentes contínuos através da inclusão. Desde que vamos comparar um número finito de autovalores podemos fazer as seguintes considerações. 
Consideraremos $\phi \in C^{2}(0,1)$ tais que $\|\phi\|_{\infty} \leq M,\left\|\phi^{\prime}\right\|_{\infty} \leq M$ e $\left\|\phi^{\prime \prime}\right\|_{\infty} \leq M \mathrm{e}$ $W^{*} \in \mathbb{R}^{n}$ tais que $\|W\|_{\infty} \leq M^{\prime}$ e $\|W\|_{H_{d}^{1}} \leq M^{\prime}$.

Teorema A.1. Sejam $\phi, \psi \in H^{1}$ e $U, V, W \in \mathbb{R}^{n}$ então:

i) $\left|\langle\phi, \psi\rangle-\left\langle P_{n}(\phi), P_{n}\langle\psi\rangle\right\rangle\right| \rightarrow 0$ quando $n \rightarrow \infty$. Assim,

se $\langle\phi, \psi\rangle=0$ então $\left\langle P_{n}(\phi), P_{n}(\psi)\right) \rightarrow 0$ quando $n \rightarrow \infty$;

se $\|\phi\|=1$ então $\left\|P_{n}(\phi)\right\| \rightarrow 1$ quando $n \rightarrow \infty$;

ii) $\left|\sum_{i=1}^{n} \frac{1}{n} f_{n}^{\prime}\left(u_{i}\right) w_{i}^{2}-\int_{0}^{1} i\left(f_{n}^{\prime}(U)\right) i(W)^{2} d x\right| \rightarrow 0$ quando $n \rightarrow \infty$;

iii) $\mid \int_{0}^{1}\left(\phi^{\prime}\right)^{2}(x) d x-\int_{0}^{1}\left\langle i\left(P_{n}(\phi)^{\prime}\right)^{2} d x\right| \rightarrow 0$ quando $n \rightarrow \infty$;

iv) $\left|\int_{0}^{1} i\left(f_{n}^{\prime}(u)\right)\left(i\left\langle P_{n} \phi\right)\right)^{2} d x-\int_{0}^{1} i\left(f_{n}^{\prime}(u)\right) \phi^{2}(x) d x\right| \rightarrow 0$;

v) $|\langle U, Y\rangle-\langle i(U), i(V)\rangle| \rightarrow 0$ quando $n \rightarrow \infty$. Assim,

se $\langle U, V\rangle=0$ então $\langle i(U), i(V)\rangle \rightarrow 0$ quando $n \rightarrow \infty$;

se $\|U\|=1$ entâa $\| i\langle U\rangle) \| \rightarrow 1$ quando $n \rightarrow \infty$;

vi) $\left|\int_{0}^{1} f^{\prime}(u) i(W)^{2} d x-\int_{0}^{1} i\left(f_{n}^{\prime}(u)\right) i(W)^{2}(x) d x\right| \rightarrow 0$ quando $n \rightarrow \infty$;

vii) $\left|\int_{0}^{1} f^{\prime}(u) i\left(P_{n} \phi\right)^{2} d x-\int_{0}^{1} f^{\prime}(u) \phi^{2}(x) d x\right| \rightarrow 0$ quando $n \rightarrow \infty$.

Observamos que por ii), vi) e vii), para $W=P_{n} w$ temos que

$$
\left|\sum_{i=1}^{n} \frac{1}{n} f^{\prime}\left(u_{i}\right) w_{i}^{2}-\int_{0}^{1} f^{\prime}(u) w^{2} d x\right|=O\left(\frac{1}{\sqrt{n}}\right) .
$$

\section{Demonstração:}

i)

$$
\langle\phi, \psi\rangle=\sum_{i=1}^{n} \int_{\frac{i-1}{n}}^{\frac{1}{n}} \phi(x) \psi(x) d x
$$

Desde que $\phi(x)=\phi\left(x_{i}\right\rangle+\phi^{\prime}\left(\bar{x}_{i}\right)\left(x-x_{i}\right), \psi(x)=\psi\left(x_{i}\right)+\psi^{\prime}\left(\tilde{x}_{i}\right)\left(x-x_{i}\right)$ para $\bar{x}_{i}$ e $\tilde{x}_{i}$ entre $x$ e $x_{i}$ então:

$$
\begin{array}{r}
\int_{\frac{i-1}{n}}^{\frac{i}{n}} \phi(x) \psi(x) d x=\int_{\frac{i-1}{n}}^{\frac{i}{n}}\left\{\phi\left(x_{i}\right) \psi\left(x_{i}\right)\right. \\
\left.\left.+\left[\Phi\left(x_{i}\right) \psi^{\prime}\left(\tilde{x}_{i}\right)+\psi\left(x_{i}\right) \phi^{\prime}\left(\bar{x}_{i}\right)\right]\left(x-x_{i}\right)+\psi^{\prime}\left(\bar{x}_{i}\right) \phi^{\prime}\left(\bar{x}_{i}\right)\right]\left(x-x_{i}\right)^{2}\right\} d x
\end{array}
$$

Então

$$
\begin{array}{r}
\left|\sum_{1=1}^{n} \int_{\frac{i-1}{n}}^{\frac{1}{n}} \phi(x) \psi(x) d x-\sum_{1}^{n} \frac{1}{n} \phi\left(x_{i}\right) \psi\left(x_{i}\right)\right| \\
\left.=\mid \sum_{i=1}^{n} \int_{\frac{1-1}{n}}^{\frac{i}{n}}\left[\phi\left(x_{i}\right) \psi^{\prime}\left(\tilde{x}_{i}\right)+\psi\left(x_{i}\right) \phi^{\prime}\left(\bar{x}_{i}\right)\right]\left(x-x_{i}\right)+\dot{\psi}^{\prime}\left(\tilde{x}_{i}\right) O^{\prime}\left(\bar{x}_{i}\right)\right]\left(x-x_{i}\right)^{2} d x \mid \\
\leq \sum_{i=1}^{n}\|\phi\|_{\infty}\left\|\psi^{\prime}\right\|_{\infty} \frac{1}{2 n^{2}}+\left\|\phi^{\prime}\right\|_{\infty}\|\psi\|_{\infty} \frac{1}{2 n^{2}}+\left\|\phi^{\prime}\right\|_{\infty}\left\|r^{\prime}\right\|_{\infty} \frac{1}{4 n^{3}}=O\left(\frac{1}{n}\right)
\end{array}
$$


ii) Como

$$
\begin{array}{r}
\int_{0}^{1} i\left(f_{n}^{\prime}(U)\right)\left(i\left(\Pi^{\circ}\right)\right)^{2} d x=\frac{1}{2 n} f^{\prime}\left(u_{1}\right) u_{1}^{2} \\
+\sum_{i=1}^{n} \int_{\frac{i-\frac{1}{2}}{n}}^{\frac{i+\frac{1}{2}}{n}}\left[f^{\prime}\left(u_{i}\right)+n\left(f^{\prime}\left(u_{i+1}\right)-f^{\prime}\left(u_{i}\right)\right)\left(x-x_{i}\right)\right] \\
.\left[w_{i}^{2}+2 n w_{i}\left(w_{i+1}-w_{i}\right)\left(x-x_{i}\right)+n^{2}\left(x-x_{i}\right)^{2}\left(w_{i+1}-w_{i}\right)^{2}\right] d x+\frac{1}{2 n} f^{\prime}\left(u_{n}\right) w_{n}^{2} .
\end{array}
$$

Calculando:

$$
\begin{array}{r}
\int_{\frac{i-\frac{1}{2}}{n}}^{\frac{i+\frac{1}{2}}{2}}\left[f^{\prime}\left(u_{i}\right)+n\left(f^{\prime}\left(u_{i+1}\right)-f^{\prime}\left(u_{i}\right)\right)\left(x-x_{i}\right)\right] \\
.\left[w_{i}^{2}+2 n w_{i}\left(w_{i+1}-w_{i}\right)\left(x-x_{i}\right)+n^{2}\left(x-x_{i}\right)^{2}\left(u_{i+1}-w_{i}\right)^{2}\right] d x \\
=\frac{1}{n} f^{\prime}\left(u_{i}\right) w_{i}^{2}+f^{\prime}\left(u_{i}\right) \frac{1}{n} w_{i}\left(w_{i+1}-w_{i}\right)+f^{\prime}\left(u_{i}\right)\left(u_{i+1}-w_{i}\right)^{2} \frac{1}{3 n} \\
+\frac{1}{2 n}\left(f^{\prime}\left(u_{i+1}\right)-f^{\prime}\left(u_{i}\right)\right) w_{i}^{2}+\frac{2}{3 n}\left(f^{\prime}\left(u_{i+1}\right)-f^{\prime}\left(u_{i}\right)\right) u_{i}\left(w_{i+1}-w_{i}\right) \\
+\frac{1}{4 n}\left(f^{\prime}\left(u_{i+1}\right)-f^{\prime}\left(u_{i}\right)\right)\left(w_{i+1}-w_{i}\right)^{2} \\
=\frac{1}{4 n} f^{\prime}\left(u_{i}\right) w_{i}^{2}+\frac{1}{6 n} f^{\prime}\left(u_{i}\right) w_{i+1} w_{i}+\frac{1}{12 n} f^{\prime}\left(u_{i}\right) w_{i+1}^{2} \\
+\frac{1}{12 n} f^{\prime}\left(u_{i+1}\right) w_{i}^{2}+\frac{1}{6 n} f^{\prime}\left(u_{i+1}\right) w_{i+1} w_{i}+\frac{1}{4 n} f^{\prime}\left(u_{i+1}\right) w_{i+1}^{2}
\end{array}
$$

Como estamos assumindo que $\|W\|_{H_{d}^{1}} \leq K$ então temos: $\left|w_{i+1}-w_{i}\right| \leq \frac{K}{\sqrt{n}}$ e assim. $w_{i+1}=w_{i}+O(1 / \sqrt{n})$, ou $w_{i}=w_{i+1}+O(1 / \sqrt{n})$. Também. $\|U\|_{\infty} \leq K$ e $\left\|W^{-}\right\|_{\infty} \leq K$ e assim.

$$
\begin{gathered}
\frac{1}{6 n} f^{\prime}\left(u_{i}\right) w_{i+1} w_{i}=\frac{1}{6 n} f^{\prime}\left(u_{i}\right) w_{i}^{2}+O\left(n^{-\frac{3}{2}}\right) \\
\frac{1}{6 n} f^{\prime}\left(u_{i+1}\right) w_{i+1} w_{i}=\frac{1}{6 n} f^{\prime}\left(u_{i+1}\right) w_{i+1}^{2}+O\left(n^{-\frac{3}{2}}\right) \\
\frac{1}{12 n} f^{\prime}\left(u_{i}\right) w_{i+1}^{2}=\frac{1}{12 n} f^{\prime}\left(u_{i}\right) w_{i}^{2}+O\left(n^{-\frac{3}{2}}\right) \\
\frac{1}{12 n} f^{\prime}\left(u_{i+1}\right) w_{i}^{2}=\frac{1}{12 n} f^{\prime}\left(u_{i+1}\right) w_{i+1}^{2}+O\left(n^{-\frac{3}{2}}\right)
\end{gathered}
$$

Portanto,

$$
\begin{array}{r}
\int_{\frac{i-\frac{1}{2}}{n}}^{\frac{i+\frac{1}{2}}{n}}\left[f^{\prime}\left(u_{i}\right)+n\left(f^{\prime}\left(u_{i+1}\right)-f^{\prime}\left(u_{i}\right)\right)\left(x-x_{i}\right)\right] \\
.\left[u_{i}^{2}+2 n w_{i}\left(w_{i+1}-w_{i}\right)\left(x-x_{i}\right)+n^{2}\left(x-x_{i}\right)^{2}\left(u_{i-1}-w_{i}\right)^{2}\right] d x \\
=\frac{1}{2 n} f^{\prime}\left(u_{i}\right) w_{i}^{2}+\frac{1}{2 n} f^{\prime}\left(u_{i+1}\right) u_{i-2}^{2}+O\left(n^{-\frac{3}{2}}\right)
\end{array}
$$


Logo,

$$
\begin{array}{r}
\int_{0}^{1} i\left(f_{n}^{\prime}(U)\right)(i(W))^{2} d x=\frac{1}{2 n} f^{\prime}\left(u_{1}\right) w_{1}^{2} \\
+\sum_{i=1}^{n} \int_{\frac{i-\frac{1}{2}}{n}}^{\frac{i+\frac{1}{2}}{2}}\left[f^{\prime}\left(u_{i}\right)+n\left(f^{\prime}\left(u_{i+1}\right)-f^{\prime}\left(u_{i}\right)\right)\left(x-x_{i}\right)\right] \\
.\left[w_{i}^{2}+2 n w_{i}\left(w_{i+1}-w_{i}\right)\left(x-x_{i}\right)+n^{2}\left(x-x_{i}\right)^{2}\left(w_{i+1}-w_{i}\right)^{2}\right] d x+\frac{1}{2 n} f^{\prime}\left(u_{n}\right) w_{n}^{2} \\
=\left(\sum_{i=1}^{n} \frac{1}{n} f^{\prime}\left(u_{i}\right) w_{i}^{2}\right)+O(1 / \sqrt{n})
\end{array}
$$

Assim,

$$
\left.\mid \int_{0}^{1} i\left(f_{n}^{\prime}(U)\right)(i(W))^{2} d x-\sum_{i=1}^{n} \frac{1}{n} f^{\prime}\left(u_{i}\right) w_{i}^{2}\right\}=O(1 / \sqrt{n}) .
$$

iii)

$$
\int_{0}^{1}\left(i\left(P_{n}(\phi)\right)^{\prime}\right)^{2} d x=\sum_{i=1}^{n} \int_{x_{i}}^{x_{i+1}} \phi^{\prime}\left(\xi_{i}\right)^{2} d x
$$

onde para alguns $\xi_{i} ; \xi_{i} \in\left(x_{i}, x_{i+1}\right)$.

Assim,

$$
\begin{array}{r}
\left|\int_{0}^{1}\left(\phi^{\prime}(x)\right)^{2} d x-\int_{0}^{1}\left(i\left(P_{n}(\phi)\right)^{\prime}\right)^{2} d x\right| \\
=\left|\int_{0}^{x_{1}}\left(\phi^{\prime}(x)\right)^{2} d x+\sum_{i=1}^{n} \int_{x_{i}}^{x_{i+1}}\left[\left(\phi^{\prime}(x)\right)^{2}-\left(\phi^{\prime}\left(\xi_{i}\right)\right)^{2}\right] d x+\int_{x_{n}}^{1}\left(\phi^{\prime}(x)\right)^{2} d x\right| \\
\leq \int_{0}^{x_{1}}\left|2 \phi^{\prime}\left(\zeta_{0}\right) \phi^{\prime \prime}\left(\zeta_{0}\right)\right| x d x+\sum_{i=1}^{n} \int_{x_{i}}^{x_{i+1}}\left|2 \phi^{\prime}\left(\zeta_{i}\right) \phi^{\prime \prime}\left(\zeta_{i}\right)\right|\left|x-\xi_{i}\right| d x \\
+\int_{x_{n}}^{1}\left|2 \phi^{\prime}\left(\zeta_{n+1}\right) \phi^{\prime \prime}\left(\zeta_{n+1}\right)\right|(1-x) d x \leq K^{2} \frac{1}{2 n^{2}}+\sum_{i=1}^{n} 2 K^{2} \frac{1}{n^{2}}=O\left(\frac{1}{n}\right)
\end{array}
$$

iv)

$$
\begin{array}{r}
1 \int_{0}^{1} i\left(f_{n}^{\prime}(U)\right)\left(i\left(P_{n}(\phi)\right)\right)^{2} d x-\int_{0}^{1} i\left(f_{n}^{\prime}(U)\right) \phi^{2} d x \mid \\
=\left|\int_{0}^{1} i\left(f_{n}^{\prime}(U)\right)\left[\left(i\left(P_{n}(\phi)\right)\right)^{2}-\phi^{2}\right] d x\right| \\
\leq\left\|f^{\prime}(u)\right\|_{\infty} \int_{0}^{1}\left|\left(i\left(P_{n}(\phi)\right)\right)^{2}-\phi^{2}\right| d x \leq 2\left\|f^{\prime}(u)\right\|_{\infty}\|\phi\|_{\infty} \int_{0}^{1}\left|i\left(P_{n}(\phi)\right)-\phi\right| d x
\end{array}
$$


Calculamos primeiramente $\int_{0}^{1}\left|\left(i\left(P_{n}(\phi)\right)\right)-\phi\right| d x$

$$
\begin{array}{r}
\int_{0}^{1}\left|\left(i\left(P_{n}(\phi)\right)\right)-\phi\right| d x=\int_{0}^{x_{1}}\left|\phi\left(x_{1}\right)-\phi(x)\right| d x \\
+\sum_{i+1}^{n} \int_{x_{i}}^{x_{i+1}}\left|\phi\left(x_{i}\right)-n\left(\phi\left(x_{i+1}\right)-\phi\left(x_{i}\right)\right)\left(x-x_{i}\right)-\phi(x)\right| d x \\
+\int_{x_{n}}^{1}\left|\phi\left(x_{n}\right)-\phi(x)\right| d x \leq \frac{2 K}{n}+\sum_{i=1}^{n} \int_{x_{i}}^{x_{i+1}}\left|\left(\phi^{\prime}\left(\zeta_{i}\right)-\phi^{\prime}\left(\varsigma_{i}\right)\right)\left(x-x_{i}\right)\right| d x \\
\leq \frac{2 K}{n}+\sum_{i=1}^{n} \frac{K}{n^{2}} \leq \frac{3 K}{n}
\end{array}
$$

Logo,

$$
\left|\int_{0}^{1} i\left(f_{n}^{\prime}(U)\right)\left(i\left(P_{n}(\phi)\right)\right)^{2} d x-\int_{0}^{1} i\left(f_{n}^{\prime}(U)\right) \phi^{2} d x\right|=\frac{6 K^{3}}{n}\left\|f^{\prime}(u)\right\|_{\infty}
$$

v)

$$
\begin{array}{r}
\int_{0}^{1} i\left(\Pi^{\prime}\right) i(Z) d x=\int_{0}^{x_{1}} w_{1} z_{1} d x+\sum_{i=1}^{n} \int_{x_{i}}^{x_{i+1}}\left[w_{i}+n\left(u_{i+1}-w_{i}\right)\left(x-x_{i}\right)\right] \\
.\left[z_{i}+n\left(z_{i+1}-z_{i}\right)\left(x-x_{i}\right)\right] d x+\int_{x_{n}}^{1} w_{n} z_{n} d x
\end{array}
$$

Para cada $i$ calcularemos

$$
\begin{array}{r}
\int_{x_{i}}^{x_{i+1}}\left[u_{i}+n\left(w_{i+1}-w_{i}\right)\left(x-x_{i}\right)\right]\left[z_{i}+n\left(z_{i+1}-z_{i}\right)\left(x-x_{i}\right)\right] d x \\
=\frac{1}{n} w_{i} \tilde{\sim}_{i}+\frac{1}{2 n} w_{i}\left(z_{i+1}-z_{i}\right)+\frac{1}{2 n} z_{i}\left(w_{i+1}-u_{i}\right)+\frac{1}{3 n}\left(u_{i-1}-u_{i}\right)\left(z_{i+1}-z_{i}\right) \\
=\frac{1}{3 n} w_{i} z_{i}+\frac{1}{6 n} u_{i} \tilde{\sim}_{i+1}+\frac{1}{6 n} u_{i-1} z_{i}+\frac{1}{3 n} w_{i+1} z_{i+1}
\end{array}
$$

Analogamente a iii) temos

$$
\begin{gathered}
w_{i+1} z_{i}=w_{i} z_{i}+O(1 / \sqrt{n}): \\
w_{i} z_{i+1}=w_{i+1} z_{i+1}+O(1 / \sqrt{n}) .
\end{gathered}
$$

E assim,

$$
\begin{array}{r}
\int_{x_{i}}^{x_{i+1}}\left[w_{i}+n\left(w_{i+1}-w_{i}\right)\left(x-x_{i}\right)\right]\left[z_{i}+n\left(z_{i+1}-z_{i}\right)\left(x-x_{i}\right)\right] d x \\
=\frac{1}{2 n} w_{i} z_{i}+\frac{1}{2 n} w_{i+1} \tilde{z}_{i-1}+O(1 / \sqrt{n})
\end{array}
$$

Portanto,

$$
\left|\int_{0}^{1} i(W) i(Z) d x-\sum_{i=1}^{n} \frac{1}{n} u_{i} z_{i}\right|=O(1 / \sqrt{n})
$$


vi)

$$
\begin{array}{r}
\left|\int_{0}^{1} i\left(f_{n}^{\prime}(U)\right) i(W)^{2} d x-\int_{0}^{1} f^{\prime}(u) i(W)^{2} d x\right| \leq\|W\|_{\infty}^{2} \int_{0}^{1}\left|i\left(f_{n}^{\prime}(U)\right)-f^{\prime}(u)\right| d x \\
\leq K^{2}\left(\int_{0}^{x_{1}}\left|f^{\prime}\left(u_{1}\right)-f^{\prime}(u)\right| d x+\int_{x_{n}}^{1}\left|f^{\prime}\left(u_{n}\right)-f^{\prime}(u)\right|\right) d x \\
\left.+\sum_{i=1}^{n} \int_{x_{i}}^{x_{i+1}}\left|f^{\prime}\left(u_{i}\right)-n\left(f^{\prime}\left(u_{i+1}\right)-f^{\prime}\left(u_{i}\right)\right)\left(x-x_{i}\right)-f^{\prime}(u)\right| d x\right) \\
\leq K^{2}\left(\frac{2 K}{n}+\sum_{i=1}^{n} \int_{x_{i}}^{x_{i+1}} \mid\left(f^{\prime \prime}\left(u\left(\zeta_{i}\right)\right) u^{\prime}\left(\zeta_{i}\right)-f^{\prime \prime}\left(u\left(\varsigma_{i}\right)\right) u^{\prime}\left(\varsigma_{i}\right)\right)\left(x-x_{i}\right) d x\right) \\
\leq K^{2}\left(\frac{2 K}{n}+\sum_{i=1}^{n} K^{2} \frac{1}{n^{2}}\right) \leq\|W\|_{\infty, d} \frac{\tilde{K}}{n}
\end{array}
$$

Assim,

$$
\left|\int_{0}^{1} i\left(f_{n}^{\prime}(U)\right) i(W)^{2} d x-\int_{0}^{1} f^{\prime}(u) i(W)^{2} d x\right|=O\left(\frac{1}{n}\right)
$$

vii)

$$
\begin{gathered}
\left|\int_{0}^{1} f^{\prime}(u) i\left(P_{n} \phi\right)^{2} d x-\int_{0}^{1} f^{\prime}(u) \phi^{2} d x\right| \leq\left\|f^{\prime}(u)\right\|_{\infty} \int_{0}^{1}\left|\left(i\left(P_{n} \phi\right)\right)^{2}-\phi^{2}\right| d x \\
\leq 2\left\|f^{\prime}(u)\right\|_{\infty}\|\phi\|_{\infty} \int_{0}^{1}\left|i\left(P_{n} \phi\right)-\phi\right| d x \leq 2\left\|f^{\prime}(u)\right\|_{\infty} K\left(\int_{0}^{x_{1}}\left|\phi_{i}-\phi(x)\right| d x\right. \\
\left.+\sum_{i=1}^{n} \int_{x_{i}}^{x_{i+1}}\left|\phi_{i}+n\left(\phi_{i+1}-\phi_{i}\right)\left(x-x_{i}\right)-\phi(x)\right| d x+\int_{x_{n}}^{1}\left|\phi_{n}-\phi(x)\right| d x\right) \\
\leq 2\left\|f^{\prime}(u)\right\|_{\infty} K\left(2 K \frac{1}{n}+\sum_{i=1}^{n} \int_{x_{i}}^{x_{i+1}}\left|\left(\phi^{\prime}\left(\zeta_{i}\right)-\phi^{\prime}\left(\varsigma_{i}\right)\right)\left(x-x_{i}\right)\right| d x\right) \\
\leq 2\left\|f^{\prime}(u)\right\|_{\infty} K\left(2 K \frac{1}{n}+2 K \frac{1}{n}\right)
\end{gathered}
$$

Portanto.

$$
\left|\int_{0}^{1} f^{\prime}(u) i\left(P_{n} \phi\right)^{2} d x-\int_{0}^{1} f^{\prime}(u) \phi^{2} d x\right|=O\left(\frac{1}{n}\right)
$$

\section{A.2 Convergência de autovalores em $n$}

Agora, estamos em condições de mostrar a convergência dos $k$ primeiros autovalores do problema

$$
-L_{n} W+\mathrm{f}^{\prime}\left(L_{n}\right) W=\lambda W
$$

para os $k$ primeiros autovalores de

$$
\Delta w+f^{\prime}(u) w=\lambda w
$$


onde $\Delta$ é o operador Laplaciano com condição de Neumann. quando $n \rightarrow \infty$.

Para isso, vamos estudar 3 problemas:

1) $-L_{n} W+\mathrm{f}^{\prime}\left(U_{n}\right) W=\lambda W$

2) $-L_{n} W^{-}+P_{n} f^{\prime}(u) W=\lambda W$

3) $\Delta w+f^{\prime}(u) w=\lambda w$.

Primeiramente, mostraremos a convergência dos autovalores dos problemas 2) e 3).

Seja $\lambda_{1}$ o $1^{\circ}$ autovalor do problema 3), então:

$$
\lambda_{1}=\min \left\{\int_{0}^{1}\left(\phi^{\prime}\right)^{2}+\int_{0}^{1} f^{\prime}(u) \phi^{2} d x ; \phi \in H^{1}(0,1) e \int_{0}^{1} \phi^{2} d x=1\right\}
$$

e $\exists \phi_{1} \in H^{1}(0,1)$ tal que $\lambda_{1}=\int_{0}^{1}\left(\phi_{1}^{\prime}\right)^{2}+\int_{0}^{1} f^{\prime}(u) \phi_{1}^{2} d x$. Assim. consideremos $\phi_{1}^{n}=P_{n} \phi_{1} \in$ $\mathbb{R}^{n}$, pelo Teorema A.1 i) temos que $\left\|\phi_{1}^{n}\right\|=1+O\left(\frac{1}{n}\right)$. Assim, pelo Teorema A.1 ii), iii), vi) e vii) temos

$$
\begin{array}{r}
\left(L_{n} \phi_{1}^{n}, \phi_{1}^{n}\right\rangle+\sum_{i=1}^{n} \frac{1}{n} f_{n}^{\prime}\left(u_{i}\right)\left(\phi_{1}^{n}\right)_{i}^{2} \\
=\int_{0}^{1}\left(i\left(\phi_{1}^{n}\right)^{\prime}\right)^{2} d x+\int_{0}^{1} i\left(f_{n}^{\prime}(u)\right) i\left(\phi_{1}^{n}\right)^{2}+O(1 / \sqrt{n}) \\
=\int_{0}^{1}\left(\phi_{1}^{\prime}\right)^{2} d x+\int_{0}^{1} f^{\prime}(u) i\left(\phi_{1}^{n}\right)^{2} d x+O(1 / \sqrt{n}) \\
=\int_{0}^{1}\left(\phi_{1}^{\prime}\right)^{2} d x+\int_{0}^{1} f^{\prime}(u) \phi_{1}^{2} d x+O(1 / \sqrt{n})=\lambda_{1}+O(1 / \sqrt{n})
\end{array}
$$

Assim,

$$
\begin{array}{r}
\lambda_{1}^{n}=\min \left\{\left(L_{n} W, W\right\rangle+\sum_{i=1}^{n} \frac{1}{n} f_{n}^{\prime}\left(u_{i}\right)(W)_{i}^{2} ; W \in \mathbb{R}\left\|\mathfrak{W}^{\top}\right\|_{L_{d}^{2}}=1\right\} \\
\leq\left(\lambda_{1}+O(1 / \sqrt{n}) \cdot(1+O(1 / n))^{-1}\right.
\end{array}
$$

Seja $\lambda_{1}^{n}$ o primeiro autovalor do problema 2), então

$$
\lambda_{1}^{n}=\min \left\{\left(L_{n} W, W\right\rangle+\sum_{i=1}^{n} \frac{1}{n} f_{n}^{\prime}\left(u_{i}\right)(W)_{i}^{2} ; \Pi \in \mathbb{R} .\|W\|_{L_{d}^{2}}=1\right\}
$$

e existe $W_{1}^{n}$ tal que $\lambda_{1}^{n}=\left\langle L_{n} W_{1}^{n}, W_{1}^{n}\right\rangle+\sum_{i=1}^{n} \frac{1}{n} f_{n}^{\prime}\left(u_{i}\right)\left(W_{1}^{n}\right)_{i}^{2}$. Consideramos $i\left(W_{1}^{n}\right)$. Observamos que $\left\|W_{1}^{n}\right\| \leq \lambda_{1}^{n}+\left\|f^{\prime}(u)\right\|_{\infty}$ e portanto limitado en $n$. Então por $r$ ) temos 
que $\left\|i\left(W_{1}^{n}\right)\right\|_{2}=1+O(1 / \sqrt{n})$. Pelo Teorema A.1 vi) e ii) temos:

$$
\begin{array}{r}
\int_{0}^{1}\left(i\left(W_{1}^{n}\right)^{\prime}\right)^{2} d x+\int_{0}^{1} f^{\prime}(u) i\left(W_{1}^{n}\right)^{2} d x \\
=\left\langle L_{n} W_{1}^{n}, W_{1}^{n}\right\rangle+\int_{0}^{1} i\left(f_{n}^{\prime}\left(u_{i}\right)\right) i\left(W_{1}^{n}\right)^{2} d x+O(1 / \sqrt{n}) \\
=\left\langle L_{n} W_{1}^{n}, W_{1}^{n}\right\rangle+\sum_{i=1}^{n} \frac{1}{n} f_{n}^{\prime}\left(u_{i}\right)\left(W_{1}^{n}\right)_{i}^{2}+O(1 / \sqrt{n})=\lambda_{1}^{n}+O(1 / \sqrt{n})
\end{array}
$$

Assim,

$$
\begin{array}{r}
\lambda_{1}=\min \left\{\int_{0}^{1}\left(\phi^{\prime}\right)^{2}+\int_{0}^{1} f^{\prime}(u) \phi^{2} d x ; \phi \in H^{1}(0,1) e \int_{0}^{1} \phi^{2} d x=1\right\} \\
\leq\left(\lambda_{1}^{n}+O(1 / \sqrt{n})\right)(1+O(1 / n))^{-1}
\end{array}
$$

Portanto, $\lambda_{1} \leq\left(\lambda_{1}^{n}+O(1 / \sqrt{n})\right)(1+O(1 / n))^{-1} \leq \lambda_{1}+O(1 / \sqrt{n})$. Logo, $\lambda_{1}^{n} \rightarrow \lambda_{1}$ quando $n \rightarrow \infty$.

Queremos mostrar a convergência de $i\left(W_{1}^{n}\right)$ para $\phi_{1}$ quando $n \rightarrow \infty$, e consequentemente $\left\|r_{1}^{r_{n}}-P_{n} \phi_{1}\right\| \rightarrow 0$.

Primeiramente, vamos mostrar que $\left(i\left(W_{1}^{n}\right)\right)_{n}$ é convergente. De fato, desde que

$$
\int_{0}^{1}\left(i\left(W_{1}^{n}\right)^{\prime}\right)^{2} d x+\int_{0}^{1} i\left(f_{n}^{\prime}\left(u_{i}\right)\right) i\left(W_{1}^{n}\right)^{2} d x=\lambda_{1}^{n}+O(1 / \sqrt{n})
$$

e $\lambda_{1}^{n} \rightarrow \lambda_{1}$ então $\int_{0}^{1}\left(i\left(W_{1}^{n}\right)^{\prime}\right)^{2} d x+\int_{0}^{1} i\left(f_{n}^{\prime}\left(u_{i}\right)\right) i\left(W_{1}^{n}\right)^{2} d x \leq M$ para $n \geq n_{0}$. Mas

$$
\int_{0}^{1} i\left(f_{n}^{\prime}\left(u_{i}\right)\right) i\left(W_{1}^{n}\right)^{2} d x \leq\left\|f^{\prime}(u)\right\|_{\infty} \int_{0}^{1} i\left(W_{1}^{n}\right)^{2} d x=\left\|f^{\prime}(u)\right\|_{\infty}\left(1+O(1 / \sqrt{n}) \leq M^{\prime}\right.
$$

para $n \geq n_{0}$. Então $\int_{0}^{1}\left(i\left(W_{1}^{n}\right)^{\prime}\right)^{2} d x$ é limitado e portanto $\left(i\left(H_{1}^{n}\right)\right)_{n}$ é uma sequência limitada em $H^{1}$. Desde que a inclusão de $H^{1}$ em $L^{2}$ é compacta então existe uma subsequência de $\left(i\left({W_{1}^{*}}_{1}^{n}\right)\right)_{n}$; que ainda será denotada por $\left(i\left(W_{1}^{n}\right)\right)_{n}$, que converge em $L^{2}$ para algum $w_{1}$. Mas $\int_{0}^{1}\left(i\left(W_{1}^{n}\right)^{\prime}\right)^{2} d x+\int_{0}^{1} i\left(f_{n}^{\prime}\left(u_{i}\right)\right) i\left(W_{1}^{n}\right)^{2} d x=\lambda_{1}^{n}+O(1 / \sqrt{n}) . i\left(f_{n}^{\prime}(U)\right) \rightarrow f^{\prime}(u)$ em $L^{2}$ e $i\left(W_{1}^{n}\right) \rightarrow u_{1}$ em $L^{2} \operatorname{logo} \int_{0}^{1}\left(i\left(W_{1}^{n}\right)^{\prime}\right)^{2} d x \rightarrow \lambda_{1}-\int_{0}^{1} f^{\prime}(u) w_{1}^{2} d x$. Desde que $\left\|i\left(\|_{1}^{n}\right)\right\|_{H^{1}}$ é convergente e $i\left(W_{1}^{n}\right)$ converge fracamente em $H^{1}$ então $i\left(W_{1}^{n}\right)$ é convergente em $H^{1}$. Para ver que $u_{1}$ é $\pm \phi_{1}$ basta observar que $\left\|w_{1}\right\|_{L^{2}}=1$ e $\int_{0}^{1}\left(w_{1}^{\prime}\right)^{2} d x+\int_{0}^{1} f^{\prime}(u) w_{1}^{2} d x=\lambda_{1}$ e que $\pm \Phi_{1}$ são os únicos com esta propriedade.

Agora vamos mostrar a convergência do segundo autovalor.

Seja $\lambda_{2}$ o segundo autovalor do problema 3), ou seja,

$$
\lambda_{2}=\min \left\{\int_{0}^{1}\left(\phi^{\prime}\right)^{2}+\int_{0}^{1} f^{\prime}(u) \phi^{2} d x ; \phi \in H^{1}(0,1), \int_{0}^{1} \phi^{2} d x=1 \text { e } \int_{0}^{1} \phi \phi_{1} d x=0\right\}
$$


e $\exists \phi_{2} \in H^{1}(0.1)$ tal que $\lambda_{2}=\int_{0}^{1}\left(\left(\phi_{2}^{n}\right)^{\prime}\right)^{2}+\int_{0}^{1} f^{\prime}(u) \phi_{2}^{n} d x$ e $\int_{0}^{1} \phi_{2} \dot{\phi}_{1} d x=0$. Assim, consideremos $\phi_{2}^{n}=P_{n} \phi_{2} \in \mathbb{R}^{n}$, pelo Teorema A.1 i) temos que $\left\|\phi_{1}^{n}\right\|=1+O\left(\frac{1}{n}\right)$ e $\left\langle\phi_{1}^{n}, \phi_{2}^{n}\right)=O\left(\frac{1}{n}\right)$ e portanto $\left\langle W_{1}^{n}, \phi_{2}^{n}\right\rangle=\left\langle W_{1}^{n}-\phi_{1}^{n}, \phi_{2}^{n}\right)+\left\langle\phi_{1}^{n}, \phi_{2}^{n}\right\rangle \rightarrow 0$ quando $n \rightarrow \infty$. Como antes, pelo Teorema A.1 ii), iii), vi) e vii) temos

$$
\begin{array}{r}
\left\langle L_{n} \phi_{2}^{n}, \phi_{2}^{n}\right)+\sum_{i=1}^{n} \frac{1}{n} f_{n}^{\prime}\left(u_{i}\right)\left(\phi_{2}^{n}\right)_{i}^{2} \\
=\int_{0}^{1}\left(i\left(\phi_{2}^{n}\right)^{\prime}\right)^{2} d x+\int_{0}^{1} i\left(f_{n}^{\prime}(u)\right) i\left(\phi_{2}^{n}\right)^{2}+O(1 / \sqrt{n}) \\
=\int_{0}^{1}\left(\phi_{2}^{\prime}\right)^{2} d x+\int_{0}^{1} f^{\prime}(u)_{i}\left(\phi_{2}^{n}\right)^{2} d x+O(1 / \sqrt{n}) \\
=\int_{0}^{1}\left(\phi_{2}^{\prime}\right)^{2} d x+\int_{0}^{1} f^{\prime}(u) \phi_{2}^{2} d x+O(1 / \sqrt{n})=\lambda_{2}+O(1 / \sqrt{n})
\end{array}
$$

Agora, consideremos $\bar{\phi}_{2}^{n}=\phi_{2}^{n}-\left\langle W_{1}^{n}, \phi_{2}^{n}\right) W_{1}^{n}, \operatorname{logo}\left\langle{W_{1}^{n}}_{1}^{n}, \tilde{\phi}_{2}^{n}\right)=0$. Assim:

$$
\begin{array}{r}
\left\langle L_{n} \tilde{\phi}_{2}^{n}, \tilde{\phi}_{2}^{n}\right\rangle+\sum_{i=1}^{n} \frac{1}{n} f_{n}^{\prime}\left(u_{i}\right)\left(\tilde{\phi}_{2}^{n}\right)_{i}^{2} \\
=\left\langle L_{n} \phi_{2}^{n}, \phi_{2}^{n}\right\rangle-2\left\langle L_{n}^{\frac{1}{2}} \phi_{2}^{n}, L_{n}^{\frac{1}{2}} I_{1}^{-n}\right\rangle\left\langle\phi_{2}^{n}: W_{1}^{n}\right\rangle \\
+\left\langle\phi_{2}^{n}, W_{1}^{n}\right)^{2}\left\langle L_{n} W_{1}^{n}, W_{1}^{n}\right\rangle+\sum_{i=1}^{n} \frac{1}{n} f_{n}^{\prime}\left(u_{i}\right)\left(\phi_{2}^{n}\right)_{i}^{2} \\
-2 \sum_{i=1}^{n} \frac{1}{n} f_{n}^{\prime}\left(u_{i}\right)\left(\phi_{2}^{n}\right)_{i}\left(W_{1}^{n}\right)_{i}\left\langle\phi_{2}^{n}: W_{1}^{n}\right\rangle+\sum_{i=1}^{n} \frac{1}{n} f_{n}^{\prime}\left(u_{i}\right)\left(I_{1}^{-n}\right)_{i}^{2}\left(\phi_{2}^{n}, W_{1}^{n}\right)^{2} \\
\leq \lambda_{2}+O(1 / \sqrt{n})+\lambda_{1}^{n}\left\langle\delta_{2}^{n}, I_{1}^{r n}\right\rangle^{2} \\
+2\left\|\dot{o}_{2}^{n}\right\|_{H_{d}^{1}}\left\|W_{1}^{n}\right\|_{H_{d}^{1}}\left|\left(\phi_{2}^{n}, W_{1}^{n}\right\rangle\right|+2\left|\left\langle\phi_{2}^{n}, W_{1}^{n}\right\rangle\right|\left\|f_{n}^{\prime}(U\rangle\right\|_{\infty}\left\|\delta_{2}^{n}\right\|_{L_{d}^{2}}\left\|W_{1}^{n}\right\|_{L_{d}^{2}} \\
\leq \lambda_{2}+h(n)
\end{array}
$$

onde $h(n) \rightarrow 0$ quando $n \rightarrow \infty$

Portanto.

$$
\begin{array}{r}
\lambda_{2}^{n}=\min \left\{\left\langle L_{n} W, W\right\rangle+\sum_{i=1}^{n} \frac{1}{n} f_{n}^{\prime}\left(u_{i}\right)(W)_{i}^{2} ; W^{-} \in \mathbb{R},\left\|W^{-}\right\|_{L_{d}^{2}}=1 \text { e }\left\langle W^{*} W_{1}^{n}\right\rangle=0\right\} \\
\leq\left(\lambda_{2}+h(n)\right) \cdot(1+g(n))^{-1}
\end{array}
$$

onde $g(n) \rightarrow 0$ quando $n \rightarrow \infty$ pois $\left\|\bar{\phi}_{2}^{n}\right\|=1+g(n)$.

Seja $\lambda_{2}^{n}$ o segundo autovalor do problema 2), então

$$
\lambda_{2}^{n}=\operatorname{nim}\left\{\left\langle L_{n} W ;, W\right\rangle+\sum_{i=1}^{n} \frac{1}{n} f_{n}^{\prime}\left(u_{i}\right)(W)_{i}^{2}: W^{\top} \in \mathbb{R},\left\|W^{\top}\right\|_{L_{d}^{2}}=1 \text { e }\left\langle W: W_{1}^{n}\right\rangle=0\right\}
$$


e existe $W_{2}^{n}$ tal que $\left\|W_{2}^{n}\right\|=1,\left\langle W_{2}^{n}, W_{1}^{n}\right\rangle=0$ e $\lambda_{2}^{n}=\left\langle L_{n} W_{2}^{n}, W_{2}^{n}\right\rangle+\sum_{i=1}^{n} \frac{1}{n} f_{n}^{\prime}\left(u_{i}\right)\left(W_{2}^{n}\right)_{i}^{2}$. Consideramos $i\left(W_{2}^{n}\right)$ entāo por v) temos que $\left\|i\left(W_{1}^{n}\right)\right\|_{2}=1+O(1 / \sqrt{n}),\left\langle i\left(W_{2}^{n}\right), i\left(W_{1}^{n}\right)\right\rangle$ $=O(1 / \sqrt{n})$ e $\left\langle i\left(W_{2}^{n}\right), \phi_{1}\right\rangle=\left\langle i\left(W_{2}^{n}\right), \phi_{1}-i\left(W_{1}^{n}\right)\right\rangle+\left\langle i\left(W_{2}^{n}\right), i\left(W_{1}^{n}\right)\right\rangle \rightarrow 0$ quando $n \rightarrow \infty$.

Como antes, pelo Teorema A.1 vi) e ii) temos:

$$
\begin{array}{r}
\int_{0}^{1}\left(i\left(W_{2}^{n}\right)^{\prime}\right)^{2} d x+\int_{0}^{1} f^{\prime}(u) i\left(W_{2}^{n}\right)^{2} d x \\
=\left\langle L_{n} W_{2}^{n}, W_{2}^{n}\right\rangle+\int_{0}^{1} i\left(f_{n}^{\prime}\left(u_{i}\right)\right) i\left\langle W_{2}^{n}\right)^{2} d x+O(1 / \sqrt{n}) \\
=\left\langle L_{n} W_{2}^{n}, W_{2}^{n}\right\rangle+\sum_{i=1}^{n} \frac{1}{n} f_{n}^{\prime}\left(u_{i}\right)\left(W_{2}^{n}\right)_{i}^{2}+O(1 / \sqrt{n})=\lambda_{2}^{n}+O(1 / \sqrt{n})
\end{array}
$$

Agora, consideremos $\widetilde{i\left(W_{2}^{n}\right)}=i\left(W_{2}^{n}\right)-\left\langle i\left(W_{2}^{n}\right), \phi_{1}\right\rangle \phi_{1}$, logo $\left\langle\phi_{1}, \widetilde{i\left(W_{2}^{n}\right)}\right\rangle=0$. Assim,

$$
\begin{array}{r}
\int_{0}^{1}\left(\widetilde{i\left(W_{2}^{n}\right)}\right)^{2} d x+\int_{0}^{1} f^{\prime}(u){\widetilde{i\left(W_{2}^{n}\right)}}^{2} d x \\
=\int_{0}^{1}\left(i\left(W_{2}^{n}\right)^{\prime}\right)^{2} d x-2 \int_{0}^{1} i\left(W_{2}^{n}\right) \phi_{1} d x \int_{0}^{1} i\left(W_{2}^{n}\right)^{\prime} \phi_{1}^{\prime} d x \\
+\left(\int_{0}^{1} i\left(W_{2}^{n}\right) \phi_{1} d x\right)^{2} \int_{0}^{1}\left(\phi_{1}^{\prime}\right)^{2} d x+\int_{0}^{1} f^{\prime}(u) i\left(W_{2}^{n}\right)^{2} d x \\
-2 \int_{0}^{1} f^{\prime}(u) \phi_{1} i\left(W_{2}^{n}\right) d x \int_{0}^{1} i\left(W_{2}^{n}\right) \phi_{1} d x+\int_{0}^{1} f^{\prime}(u)\left(\phi_{1}\right)^{2} d x\left(\int_{0}^{1} i\left(W_{2}^{n}\right) \phi_{1} d x\right)^{2} \\
\leq \lambda_{2}^{n} O(1 / \sqrt{n})+\lambda_{1}\left(\int_{0}^{1} i\left\langle W_{2}^{n}\right) \phi_{1} d x\right)+2\left|\int_{0}^{1} i\left(W_{2}^{n}\right) \phi_{1} d x\right|\left\|\phi_{1}\right\|_{H^{1}}\left\|i\left(W_{2}^{n}\right)\right\|_{H^{1}} \\
+2\left|\int_{0}^{1} i\left(W_{2}^{n}\right) \phi_{1} d x\right|\left\|f^{\prime}(u)\right\|_{\infty}\left\|\phi_{1}\right\|_{L^{2}}\left\|i\left(W_{2}^{n}\right)\right\|_{L^{2}}=\lambda_{2}^{n}+h_{1}(n)
\end{array}
$$

onde $h_{1}(n) \rightarrow 0$ quando $n \rightarrow \infty$.

Assim,

$$
\begin{array}{r}
\lambda_{2}=\min \left\{\int_{0}^{1}\left(\phi^{\prime}\right)^{2}+\int_{0}^{1} f^{\prime}(u) \phi^{2} d x ; \phi \in H^{1}(0,1), \int_{0}^{1} \phi^{2} d x=1 \text { e } \int_{0}^{1} \phi_{1} \phi d x=0\right\} \\
\leq\left(\lambda_{2}^{n}+g_{1}(n)\right)
\end{array}
$$

onde $g_{1}(n) \rightarrow 0$ quando $n \rightarrow \infty$ Portanto, $\lambda_{2} \leq\left(\lambda_{2}^{n}+g_{1}(n)\right) \leq \lambda_{2}+g_{2}(n)$. Logo. $\lambda_{2}^{n} \rightarrow \lambda_{2}$ quando $n \rightarrow \infty$.

De forma análoga a anterior, mostra-se que $i\left(W_{2}^{n}\right) \rightarrow \phi_{2}$.

Por indução, temos que os $k$ primeiros autovalores e as $K$ primeiras autofunções dos problema 2) convergem para os $K$ primeiros autovalores e as $k$ primeiras autofunçōes dos problema 3) quando $n \rightarrow \infty$ 
Observando que os pontos de equilíbrio $\left(U_{n}\right)_{j}$ convergem para os equilíbrios $u_{j}$, para $j=1, \cdots, N$ dado que os campos da equação do calor e sua discretização estão $C^{1}$ suficientemente próximos para $n$ suficientemente grande. Então, de forma análoga, obtemos a convergência dos autovalores e autofunções, dos problemas 1) e 2).

\section{A.3 Convergência de autovalores em $\eta$ e $n$}

Como já foi mostrado no capítulo (1), temos que os pontos de equilíbrio para os problemas (4.3) e (4.4) são os mesmos para qualquer valor do parâmetro $\eta>0$. Estes pontos de equilíbrio são dados por: $Y_{n}=\left(U_{n}, 0\right)$ onde $U_{n}$ satisfaz $A_{n} U_{n}+\mathrm{f}\left(U_{n}\right)=0$.

Fixemos um ponto de equilíbrio $\left(Y_{n}\right)_{j}$, assim não escreveremos mais o índice $j$. Consideramos as equações linearizadas em $Y_{n}$, ou seja,

$$
\frac{d}{d t}\left[\begin{array}{l}
U \\
V
\end{array}\right]=\left[\begin{array}{cc}
0 & I \\
-A_{n} & -2\left(\eta A_{n}^{1 / 2}+a\right)
\end{array}\right]\left[\begin{array}{l}
U \\
V
\end{array}\right]+\left[\begin{array}{c}
0 \\
\mathrm{f}^{\prime}\left(U_{n}\right) U
\end{array}\right]
$$

Denotaremos por $C_{n}^{\prime}\left(U_{n}\right)=\left[\begin{array}{cc}0 & I \\ -A_{n} & -2 a\end{array}\right]+\left[\begin{array}{cc}0 & 0 \\ \mathrm{f}^{\prime}\left(U_{n}\right) & 0\end{array}\right]$

e $A_{\eta, n}^{\prime}\left(U_{n}\right)=\left[\begin{array}{cc}0 & I \\ -A_{n} & -2\left(\eta A_{n}^{1 / 2}+a\right)\end{array}\right]+\left[\begin{array}{cc}0 & 0 \\ \mathrm{f}^{\prime}\left(U_{n}\right) & 0\end{array}\right]$

Mostraremos que os autovalores de $C_{\eta, n}^{\prime}\left(U_{n}\right)$ convergem para os autovalores de $C_{n}^{\prime}\left(U_{n}\right)$ quando $\eta \rightarrow 0$ e a convergência é uniforme em $n$ sobre compactos. Para isto, faremos a seguinte separação de $C_{\eta, n}^{\prime}\left(U_{n}\right)$.

$$
C_{\eta, n}^{\prime}\left(U_{n}\right)=C_{n}^{\prime}\left(U_{n}\right)+B_{\eta, n},
$$

onde $B_{\eta}=\left[\begin{array}{cc}0 & 0 \\ 0 & -2 \eta A_{n}^{1 / 2}\end{array}\right]$. Observamos primeiramente que $B_{\eta, n}$ é $C_{n}^{\prime}\left(U_{n}\right)$-relativamente limitado. De fato,

$$
\left\|B_{\eta, n}\left(\left[\begin{array}{l}
U \\
V
\end{array}\right]\right)\right\|_{\mathbb{R}^{n} \times \mathbb{R}^{n}}=2 \eta\left\|A_{n}^{1 / 2} V\right\|_{L_{d}^{2}}=2 \eta\|V\|_{H_{d}^{1}} \leq 2 \eta\left\|C_{n}^{\prime}\left(U_{n}\right)\left(\left[\begin{array}{l}
U \\
V
\end{array}\right]\right)\right\|_{\mathbb{R}^{n} \times \mathbb{R}^{n}}
$$

Como, para $\lambda \in \rho\left(C_{n}^{\prime}\left(U_{n}\right)\right)$ e $\lambda$ tal que $\left\|B_{\eta, n}\left(\lambda-C_{n}^{\prime}\left(U_{n}\right)\right)^{-1}\right\|<1$, temos $\lambda \in \rho\left(C_{\eta, n}^{\prime}\left(U_{n}\right)\right)$ pois $\left(\lambda I-C_{\eta, n}^{\prime}\left(U_{n}\right)\right)^{-1}=\left(I-B_{\eta, n}\left(\lambda-C_{n}^{\prime}\left(U_{n}\right)\right)^{-1}\right)^{-1}\left(\lambda-C_{n}^{\prime}\left(U_{n}\right)\right)^{-1}$. É claro que dado 
$\lambda \in \rho\left(C_{n}^{\prime}\left(U_{n}\right)\right)$ existe um $\eta_{0}(\lambda, n)$ tal que $\lambda \in \rho\left(C_{\eta, n}^{\prime}\left(U_{n}\right)\right)$ para $\eta \leq \eta_{0}$ pois:

$$
\left\|B_{\eta, n}\left(\lambda-C_{n}^{\prime}\left(U_{n}\right)\right)^{-1}\right\|<2 \eta\left(1+|\lambda|\left\|R\left(\lambda, C_{n}^{\prime}\left(U_{n}\right)\right)\right\| .\right.
$$

Queremos mostrar que dado $\epsilon>0$, existem $\eta_{0}$ e $n_{0}$ tal que para $\eta \leq \eta_{0}$ e $n \geq n_{0}$ temos que $K_{\epsilon} \subset \rho\left(C_{\eta, n}^{\prime}\left(U_{n}\right)\right)$, onde $K_{\epsilon}=B_{K} \backslash \bigcup_{j} B_{\epsilon}\left(\mu_{j}\right)$ e $\mu_{j} \in \sigma\left(C^{\prime}(\phi)\right)$, onde $\phi$ é o limite de $U_{n}$.

Denotamos por $A^{\prime}(\phi)$ e $A_{n}^{\prime}\left(U_{n}\right)$ os operadores $\Delta-f^{\prime}(\phi)$ e $-L_{n}-f^{\prime}\left(U_{n}\right)$, respectiramente. Os autovalores de $C^{\prime}(\phi)$ são da forma

$$
\mu_{j \pm}=-a \pm \sqrt{a^{2}-\nu_{j}}
$$

onde $\nu_{j}$ é o j-ésimo autovalor de $-A^{\prime}(\phi)$. De forma análoga, temos que os autovalores de $C_{n}^{\prime}\left(U_{n}\right)$ são da forma

$$
\mu_{j \pm}^{n}=-a \pm \sqrt{a^{2}-\nu_{j}^{n}}
$$

onde $\nu_{j}^{n}$ é o j-ésimo autovalor de $-A_{n}^{\prime}\left(U_{n}\right)$.

Desde que os autovalores de $A_{n}^{\prime}\left(U_{n}\right)$ convergem para os autovalores de $A^{\prime}(\phi), \nu_{j}^{n} \rightarrow \nu_{j}$ uniforme para um número finito então $\mu_{j \pm}^{n} \rightarrow \mu_{j \pm}$ quando $n \rightarrow \infty$, uniformemente para $\mu_{j \pm}$ em $B_{K}$.

Observamos que basta mostrar que $\left\|R\left(\lambda, C_{n}^{\prime}\left(U_{n}\right)\right)\right\| \leq M$, com $M$ independente de $\eta$ e de $n$ para $\eta \leq \eta_{0}$ e $n \geq n_{0}$ e $\lambda \in K_{\epsilon}$.

Para cada $n$. seja $\phi_{j}^{n}$ o autovetor de $-A_{n}^{\prime}\left(C_{n}\right)$ associado a $\nu_{j}^{n}$. Os autovetores $\phi_{j}^{n}$ formam uma base ortogonal em $\mathbb{R}^{n}$. Os autovetores de $C_{n}^{\prime}\left(C_{n}\right)$ são da forma: $\psi_{j \pm}^{n}=$ $\left(\phi_{j}^{n}, \mu_{j \pm}^{n} \phi_{j}^{n}\right)$. Como estamos interessados no cálculo do operador resolvente então devemos considerar o operador $C_{n}^{\prime}\left(U_{n}\right)$ como um operador sobre $\mathbb{C}^{n} \times \mathbb{C}^{n}$ e $\psi_{j \pm}^{n}=\left(\phi_{j}^{n}, \mu_{j \pm}^{n} \phi_{j}^{n}\right)_{j \pm}$ é uma base para $\mathbb{C}^{n} \times \mathbb{C}^{n}$. Por trabalharmos com esta nova base, vamos considerar uma nova norma a qual é equivalente a norma original e com constantes de equivalência independentes de $n$. Seja $\lambda \gg 1$ entāo definimos a seguinte norma

$$
\left\|\left(K^{\prime} Z\right)\right\|=\left(\left(\left(-A^{\prime}\left(U_{n}\right)+\lambda\right) W, \bar{W}\right)+\langle Z \cdot \bar{Z}\rangle\right)^{\frac{1}{2}} .
$$

Esta norma é proveniente do produto interno

$$
\left\langle\left(W_{1}^{*}, Z_{1}\right) \cdot\left(W_{2}^{\prime}, Z_{2}\right)\right\rangle=\left\langle\left(-A^{\prime}\left(U_{n}\right)+\lambda\right) W_{1}, H_{2}^{\circ}\right\rangle+\left\langle Z_{1}, Z_{2}\right\rangle,
$$


observamos que a notação $\langle$,$\rangle denota o produto interno \left\langle U, V^{\prime}\right\rangle=\sum_{i=1}^{n} \frac{1}{n} u_{i} \bar{v}_{i}$, ou seja, o produto interno $L^{2}$ discretizado sobre $\mathbb{C}^{n}$. Também temos que para $j \neq l$,

$$
\left\langle\psi_{j \pm}^{n}, \psi_{l \pm}^{n}\right\rangle=\left\langle\left(\nu_{j}^{n}+\lambda\right) \phi_{j}^{n}, \phi_{l}^{n}\right\rangle+\left\langle\mu_{j \pm}^{n} \phi_{j}^{n}, \mu_{l \pm}^{n} \phi_{l}^{n}\right\rangle=0
$$

Ou seja, temos que os auto-espaços dois dimensional, associados aos autovalores \pm , são ortogonais entre si.

Além disso, o 'ângulo' formado entre os vetores da base de cada espaço dois dimensional não vai para zero quando $j \rightarrow \infty$ De fato, calculemos primeiramente

$$
\begin{gathered}
\left\langle\psi_{j+}^{n}, \psi_{j-}^{n}\right)=\left\langle\left(\nu_{j}^{n}+\lambda\right) \phi_{j}^{n}, \phi_{j}^{n}\right\rangle+\left\langle\mu_{j+}^{n} \phi_{j}^{n}, \mu_{j-}^{n} \phi_{j}^{n}\right\rangle \\
=\left(\nu_{j}^{n}+\lambda+\mu_{j+}^{n} \overline{\mu_{j-}^{n}}\right)\left\|\phi_{j}^{n}\right\|^{2} \\
\left\|\psi_{j+}^{n}\right\|^{2}=\left(\nu_{j}^{n}+\lambda+\mu_{j+}^{n} \overline{\mu_{j+}^{n}}\right)\left\|\phi_{j}^{n}\right\|^{2} \\
\left\|\psi_{j-}^{n}\right\|^{2}=\left(\nu_{j}^{n}+\lambda+\mu_{j-}^{n} \overline{\mu_{j-}^{n}}\right)\left\|\phi_{j}^{n}\right\|^{2}
\end{gathered}
$$

Como, $\mu_{j+}^{n}=\overline{\mu_{j-}^{n}}$ então $\mu_{j+}^{n} \overline{\mu_{j-}^{n}}=a^{2}-\left(\nu_{j}^{n}-a^{2}\right)-2 a \sqrt{\nu_{j}^{n}-a^{2}} i \operatorname{logo}\left(\nu_{j}^{n}+\lambda+\mu_{j+}^{n} \overline{\mu_{j-}^{n}}\right)=$ $\left(2 a^{2}+\lambda\right)-2 a \sqrt{\nu_{j}^{n}-a^{2}} i$. Também, $\left|\mu_{j+}^{n}\right|^{2}=\left|\mu_{j-}^{n}\right|^{2}=\nu_{j}^{n} \operatorname{logo},\left(\nu_{j}^{n}+\lambda+\mu_{j-}^{n} \overline{\mu_{j+}^{n}}\right)^{\frac{1}{2}}\left(\nu_{j}^{n}+\right.$ $\left.\lambda+\mu_{j-}^{n} \overline{\mu_{j-}^{n}}\right)^{\frac{1}{2}}=2 \nu_{j}^{n}+\lambda$. Então:

$$
\frac{\left\langle\psi_{j+}^{n}, \psi_{j-}^{n}\right)}{\left\|\psi_{j+}^{n}\right\|\left\|\psi_{j-}^{n}\right\|}=\frac{\left(2 a^{2}+\lambda\right)-2 a \sqrt{\nu_{j}^{n}-a^{2}} i}{2 \nu_{j}^{n}+\lambda}
$$

Logo, quando $\nu_{j}^{n} \rightarrow \infty$ temos que

$$
\frac{\left\langle\psi_{j+}^{n}, \psi_{j-}^{n}\right)}{\left\|\psi_{j+}^{n}\right\|\left\|\psi_{j-}^{n}\right\|} \rightarrow 0+0 i
$$

Agora, vamos calcular $R\left(\xi, C_{n}^{\prime}\left(U_{n}\right)\right) \psi_{j \pm}$ para $\xi \in K_{\epsilon}$.

$$
\left(\xi I-C_{n}^{\prime}\left(U_{n}\right)\right) \psi_{j \pm}^{n}=\left(\xi-\mu_{j \pm}^{n}\right) \psi_{j \pm}^{n}
$$

Logo, $\left\|\left(\xi I-C_{n}^{\prime}\left(U_{n}\right)\right) \psi_{j \pm}^{n}\right\|=\mid \xi-\mu_{j \pm}^{n}\|\| \psi_{j \pm}\|\geq \epsilon\| \psi_{j \pm} \|$ e portanto $\left\|R\left(\xi, A^{\prime}\left(U_{n}\right)\right)\right\| \leq$ $\frac{1}{\epsilon}\left\|\psi_{j \pm \|}\right\|$

Calcularemos $\left\|R\left(\xi, C_{n}^{\prime}\left(U_{n}\right)\right)(W, Z)\right\|$ para qualquer $(W, Z) \in \mathbb{C}^{n} \times \mathbb{C}^{n}$ e $\xi \in K_{\epsilon}$. Para isso escrevemos $(W, Z)$ na base de $\mathbb{C}^{n} \times \mathbb{C}^{n}$, ou seja, $(W, Z)=\sum_{j=1}^{n}\left(C_{j+} \psi_{j+}^{n}+C_{j-} \psi_{j-}^{n}\right)$, 
observamos que a soma em $j$ é ortogonal. Assim,

$$
\begin{array}{r}
\left\|R\left(\xi, C_{n}^{\prime}\left(C_{n}\right)\right)(W, Z)\right\|^{2}=\sum_{j=1}^{n}\left\|C_{j+} R\left(\xi, C_{n}^{\prime}\left(U_{n}\right)\right) \psi_{j+}^{n}+C_{\jmath-} R\left(\xi, C_{n}^{\prime}\left(U_{n}\right) \psi_{j-}^{n}\right)\right\|^{2} \\
=\sum_{j=1}^{n}\left\|C_{j+}\left(\xi-\mu_{j+}^{n}\right)^{-1} \psi_{j+}^{n}+C_{j-}\left(\xi-\mu_{j-}^{n}\right)^{-1} \psi_{j-}^{n}\right\|^{2} \\
\leq K^{2} \sum_{j=1}^{n}\left(\left\|C_{j+}\left(\xi-\mu_{j+}^{n}\right)^{-1} \psi_{j+}^{n}\right\|+\left\|C_{j-}\left(\xi-\mu_{j-}^{n}\right)^{-1} \psi_{j-}^{n}\right\|\right)^{2} \\
\leq K^{2} \max _{j= \pm 1, \pm 2, \cdots, \pm n}\left|\xi-\mu_{j+}^{n}\right|^{-1} \sum_{j=1}^{n}\left(\left\|C_{j+} \psi_{j+}^{n}\right\|+\| C_{j-}\left(\psi_{j-}^{n} \|\right)^{2}\right. \\
\leq K^{2} K^{\prime 2} \frac{1}{\epsilon} \sum_{j=1}^{n}\left(\left\|C_{j+} \psi_{j+}^{n}+\right\| C_{j-}\left(\psi_{j-}^{n}\right)^{2}=K^{2} K^{2} \frac{1}{\epsilon}\|(W, Z)\|\right.
\end{array}
$$

Portanto, $\left\|R\left(\xi . C_{n}^{\prime}\left(U_{n}\right)\right)\right\| \leq M$ para $\xi \in K_{\epsilon}$ e para todo $n \geq n_{0}$. Logo, $2 \eta(1+$ $\left.|\lambda|\left\|R\left(\xi, C_{n}^{\prime}\left(U_{n}\right)\right)\right\| \mid\right) \leq 1$, para $n \geq n_{0}$ e $\eta \leq \eta_{0}$.

Observamos que acima foi utilizado que as normas do produto interno e da soma são equivalentes com constantes de equivalência independentes de $j$ e de $n$. Agora faremos a demonstração deste fato.

Teorema A.2. As normas da soma e do produto interno são equivalentes independentes de $j$ e de $n$, ou seja, existem $K$ e $K^{\prime}$ positivos tais que se $(\Pi ; Z)=\alpha \psi_{j+}^{n}+\beta \psi_{j-}^{n}$ então

$$
K^{\prime}\left(\left\|\alpha v_{j++}^{n}\right\|+\left\|\beta \psi_{j-}^{n}\right\|\right) \leq\|(W, Z)\| \leq K\left(\left\|\alpha \psi_{j+}^{n}\right\|+\left\|\beta \psi_{j-}^{n}\right\|\right)
$$

Demonstração: A desigualdade do lado direito é trivial pois

$$
\begin{array}{r}
\left\|\left(W^{*} Z\right)\right\|=\left\|\alpha \psi_{j+}^{n}\right\|^{2}+2 \operatorname{Re}\left(\left\langle\alpha \psi_{j+}^{n}, \beta \psi_{j-}^{n}\right)\right)+\left\|\beta \psi_{j-}^{n}\right\|^{2} \\
\leq\left\|\alpha \psi_{j+-}^{n}\right\|^{2}+2\left|\left\langle\alpha \psi_{j+}^{n}, \beta \psi_{j-}^{n}\right)\right|+\left\|\beta \psi_{j-}^{n}\right\|^{2} \\
\leq\left\|\alpha \psi_{j+}^{n}\right\|^{2}+2\left\|\alpha \psi_{j+}^{n}\right\|\left\|\beta \psi_{j-}^{n}\right\|+\left\|3 \psi_{j-}^{n}\right\|^{2} \\
\leq\left(\left\|\alpha \psi_{j+}^{n}\right\|+\left\|3 v_{j-}^{n}\right\|\right)^{2}
\end{array}
$$

Antes de fazer a demonstração da desigualdade do lado esquerdo, faremos algumas considerações. Consideraremos $\psi_{j \pm}^{n}$ normalizados. Seja $r=\min _{|\alpha|+|\beta|=1}\left\|\alpha \psi_{j+}^{n}+\beta \psi_{j-}^{n}\right\|=$ $\left.\min _{|\alpha|+|\beta|=1}\left|\alpha_{\mid}^{2}+2 \operatorname{Re}\left(\left\langle\alpha v_{j+}^{n}, \beta \psi_{j-}^{n}\right\rangle\right)+\right| \beta\right|^{2}$ então $r \geq r_{0}>0$ onde $r_{0}$ independe de $j$ e de 
n. De fato, como

$$
\begin{array}{r}
\frac{2 \operatorname{Re}\left(\left\langle\alpha \psi_{j+}^{n}, \beta \psi_{j-}^{n}\right\rangle\right)}{|\alpha|^{2}+|\beta|^{2}} \\
=\frac{2 \operatorname{Re}(\alpha \bar{\beta})}{|\alpha|^{2}+|\beta|^{2}} \operatorname{Re}\left(\left\langle\psi_{j+}^{n}, \psi_{j-}^{n}\right\rangle\right)-\frac{2 \operatorname{Im}(\alpha \bar{\beta})}{|\alpha|^{2}+|\beta|^{2}} \operatorname{Im}\left(\left(\psi_{j+}^{n}, \psi_{j-}^{n}\right\rangle\right) \\
=\frac{2 \operatorname{Re}(\alpha \bar{\beta})}{|\alpha|^{2}+|\beta|^{2}} \frac{2 a^{2}+\lambda}{2 \nu_{j}^{n}+\lambda}+\frac{2 \operatorname{Im}(\alpha \bar{\beta})}{|\alpha|^{2}+|\beta|^{2}} \frac{2 a \sqrt{\nu_{j}^{n}-a^{2}}}{2 \nu_{j}^{n}+\lambda} \rightarrow 0
\end{array}
$$

Portanto, para $j$ e $n$ suficientemente grandes temos que

$$
\frac{2 \operatorname{Re}\left(\left\langle\alpha \psi_{j+}^{n}, \beta \psi_{j-}^{n}\right\rangle\right)}{|\alpha|^{2}+|\beta|^{2}} \geq-1+r_{0}
$$

$\mathrm{E}$ assim, $\left.|\alpha|^{2}+2 \operatorname{Re}\left(\left\langle\alpha \psi_{j+}^{n}, \beta \psi_{j-}^{n}\right\rangle\right)+|\beta|^{2} \geq r_{0}\left(|\alpha|^{2}+|\beta|^{2}\right\rangle \geq \tilde{r_{0}}\right)$.

Agora mostraremos que $B_{(\ldots, .)}(r) \subset B_{S}(1)$ onde $B_{(., .)}(r)$ denota a bola de raio $r$ na norma do produto interno e $B_{S}(1)$ denota a bola de raio 1 na norma da soma. De fato, suponha, por absurdo que existe $\Phi$ tal que $\|\Phi\|_{(., .)}=r$ e tal que $\|\Phi\|_{S}>1$ então considere $\Psi=\frac{\Phi}{\|\Phi\|_{s}}$ assim, $\|\Psi\|_{S}=1$ e $\|\Psi\|_{(. . .)}=\frac{\|\Phi\|_{(\ldots)}}{\|\Phi\|_{S}} \leq r$ absurdo pois $r=\min \left\{\|\Phi\|_{(., .)} ;\|\Phi\|_{S}=1\right\}$. Logo, $\|\Phi\|=1$ implica que $\|\Phi\|_{(., .)} \geq r$ e portanto $r\|\Phi\|_{S}=r \leq\|\Phi\|_{(., .)}$. e $r \geq r_{0}>0$ com $r_{0}$ independente de $j$ e de $n$. 


\section{Referências Bibliográficas}

[ACR1] J. Arrieta, A. N. Carvalho and A. Rodriguez-Bernal, Attractors for Parabolic Problems with Nonlinear Boundary Conditions. Uniform Bounds, Communications in Partial Differential Equations, 25, (2000).

[ACR2] J. M. Arrieta, A. N. Carvalho and A. Rodríguez-Bernal, Upper Semicontinuity of Attractors for Parabolic Problems with Localized Large Diffusion and Nonlinear Boundary Conditions, aceito para publicação no Journal of Differential Equations.

[C] A. ‥ Carvalho, Reaction-Diffusion Problems with Nonlinear Boundary Conditions in Cell Tissues, Resenhas, Vol. 03, no. 01, pp. 125-140 (1997).

[CDR] A. N. Carvalho, T. Dlotko and H. M. Rodrigues, Upper Semicontinuity of Attractors and Synchronization, J. Math. Anal. Appl, Vol. 220, pp. 13-41, (1998).

[CH] A. ․ Carvalho and G. Hines, Lower Semicontinuity of Unstable Manifolds for Gradient Systems, Dynamic Systems and Applications, Vol. 9, $\mathrm{n}^{\circ}$ 1: pp 37-50. (2000).

[CP] A. N. Carvalho and A. L. Pereira, A Scalar Parabolic Equation Whose Asymptotic Behavior is Dictated by a System of Ordinary Differential Equations, Journal of Differential Equations, Vol. 112, No. 1, (1994).

[CHo] R. G. Casten and C. J. Holland, Instability Results for Reaction Diffusion Equations with Neumann Boundary Conditions, Journal of Differential Equations, Vol. 27, pp. 266-273, (1978). 
[Ch] N. Chafee, Asymptotic Behavior for Solutions of a One-Dimensional Parabolic Equation with Homogeneous Neumann Boundary Conditions, Journal of Differential Equations, Vol. 18; pp. 111-134, (1975).

[CI] $\quad$. Chafee and E. F. Infante, A bifurcation problem for a nonlinear partial differential equation of parabolic type, Appl. Anal., Vol. 4, pp. 17-31, (1974).

[CT1] S. Chen e R. Triggiani, Proof of Extensions of two Conjectures on Structural Damping for Elastic Systems, Pacific Journal of Mathematics, Vol 136, $\mathrm{n}^{\circ} 1$, $15-55(1989)$.

[CT2] S. Chen e R. Triggiani, Characterization of Domains of Fractional Powers of Certain Operators Arising in Elastic Systems, and Applications, Journal of Differential Equations, Vol. 88, 279-293 (1990).

[E] M. A. Evgrafov, Asymptotic Estimates and Entire Functions, Gordon and Breach, New York (1961).

[FO] G. Fusco and W. M. Oliva, Jacobi matrices and transversality, Proceedings of the Royal Society of Edinburgh, 109A, 231-243, (1988).

[FR] B. Fiedler and C. Rocha, Orbit Equivalence of Global Attractors of Semilinear Parabolic Differential Equations, Transactions of the American Mathematical Societr, 352, $\mathrm{n}^{\circ} 1$, pp 257-284, (2000).

[Ha1] J. K. Hale, Numerical and Dynamics, Chaotic Numerics, Contemporary Mathematics, Vol. 172, pp. 1-30. A.M.S. (1994).

[Ha2] J. K. Hale, Asvmptotic Behavior of Dissipative systems, Mathematical Surveys and Monographs, Vol 25. AMS, (1988).

[Ha3] J. K. Hale, Ordinary Differential Equations, John Wiley \& Sons, (1969).

[HMO] J. K. Hale, L. T. Magalhães e W. M. Oliva. An Introduction to Infinite Dimensional Dynamical Systems - Geometric Theorv, Applied Mathematical Sciences, Vol 47; Springer-Verlag, (1984). 
[HR1] J. K. Hale e G. Raugel, Upper Semicontinuity of Attractor for a Singularly Perturbed Hyperbolic Equation, Journal of Differential Equations, Volume 73, no. 2, pp. 197-214 (1988)

[HR2] J. K. Hale e G. Raugel, Lower Semicontinuity of Attractors of Gradient Systems and Applications, Ann. Mat. Pura and Appl., (4), 154, 281-326, (1989).

[He1] D. Henry, Some Infinite-Dimensional Morse-Smale Systems Defined by Parabolic Partial Differential Equations, Journal of Differential Equations, Vol. 59, (1985).

[He2] D. Henry, Geometric theory of semilinear parabolic equations, Lecture Notes in Mathematics 840, Springer-Verlag, Berlin, (1981).

[M] Mora, X.; Finite-dimensional attracting invariant manifolds for damped semilinear wave equations, Contributions do nonllinear partial differential equations, volume II, pp 172-183, Paris (1985).

[MSM] Mora, X.; Sola-Morales, J., Existence and nonexistence of finite-dimensional globally attracting invariant manifolds in semilinear damped wave equations. Dynamics of infinite-dimensional systems, NATO Adv. Sci. Inst. Ser. F: Comput Systems Sci., 37, pp 187-210, Springer, Berlin-New York (1987).

[Ro] C. Rocha, Bifurcations in Discretized Reaction-Diffusion Equations, Resenhas IME-USP, 1, 403-419, (1994).

[Sm] G. D. Smith, Numerical Solution of Partial Differential Equations - Finite Difference Methods, Oxford University Press, (1978). 


\section{ERRATA}

Página 4, última linha trocar $A u_{x x}$ por $A u$.

Página 6, linha 2, trocar

$$
C_{\eta \theta}=\left[\begin{array}{cc}
0 & I \\
-A & -2\left(A^{\theta}+a\right)
\end{array}\right]
$$

por

$$
C_{\eta \theta}=\left[\begin{array}{cc}
0 & I \\
-A & -2\left(\eta A^{\theta}+a\right)
\end{array}\right] .
$$

Página 30 , linha -5 , trocar convegência por convergência.

Página 40, linha 12, trocar $\phi_{3, \lambda}$ por $\phi_{N, \lambda}$.

Página 51, linhas 2 e 4, trocar $T_{\lambda}$ por $T_{L_{\lambda}}$.

Página 63, linha 7, trocar $s(n)=\|\| \tilde{\sigma}_{n}\|\|=\sup _{v_{n} \in \mathbb{R}^{n}}\left\{\tilde{\sigma}_{n}\left(v_{n}\right)\right\}$ por $s(n)=\left\|\tilde{\sigma}_{n}\right\| \|=$ $\sup _{v_{n} \in \mathbb{R}^{n}}\left\{\left\|\tilde{\sigma}_{n}\left(v_{n}\right)\right\|\right\}$.

Página 75 , linha 8 , trocar

$$
2 b\left\langle f\left(u_{\eta}(t)\right), u_{\eta}(t)\right\rangle \leq-\frac{\delta}{4}\left\|u_{\eta}(t)\right\|_{L^{2}}^{2}+c_{\delta / 4}
$$

por

$$
2 b\left\langle f\left(u_{\eta}(t)\right), u_{\eta}(t)\right) \leq 2 b\left(-\frac{\delta}{4}\left\|u_{\eta}(t)\right\|_{L^{2}}^{2}+c_{\delta / 4}\right) .
$$

Página 78, linha 3, retirar $i=0,1$.

Página 78 , linha -5 , trocar "então pela unicidade então" por "então pela unicidade temos".

Página 84, linha -9, trocar $A_{\eta}^{\prime}\left(\Phi_{j}\right), \sigma\left(A_{\eta}^{\prime}\left(\Phi_{j}\right)\right)$ por $C_{\eta}^{\prime}\left(\Phi_{j}\right), \sigma\left(C_{\eta}^{\prime}\left(\Phi_{j}\right)\right)$.

Página 87, linha 6, trocar $A\left[\begin{array}{l}u \\ v\end{array}\right]$ por $C\left[\begin{array}{l}u \\ v\end{array}\right]$

Página 89, linha 1 trocar "sem-continuidade" por "semi-continuidade".

Página 94, linha 18, trocar

$$
2 b\left\langle\mathrm{f}\left(U_{\eta, n}(t)\right), U_{\eta, n}(t)\right\rangle \leq-\frac{\delta}{4}\left\|U_{\eta, n}(t)\right\|_{L_{d}^{2}}^{2}+c_{\delta / 4}
$$

por

$$
2 b\left\langle\mathrm{f}\left(U_{\eta, n}(t)\right), U_{\eta, n}(t)\right) \leq 2 b\left(-\frac{\delta}{4}\left\|U_{\eta, n}(t)\right\|_{L_{d}^{2}}^{2}+c_{\delta / 4}\right)
$$


Página 11 0 , lunha 9, trocar $e^{\operatorname{Re}\left(\lambda_{ \pm k}^{n}\right) t} \leq \epsilon t^{-\beta}$ por $e^{\operatorname{Re}\left(\lambda_{ \pm k}\right) t} \leq \epsilon t^{-\beta}$.

Página 116, linha 1, trocar $\lambda_{ \pm k}=\alpha_{k} \pm \beta_{k}$ e $\lambda_{ \pm k}^{n}=\alpha_{k}^{n} \pm \beta_{k}^{n}$ por $\lambda_{ \pm k}=\alpha_{k} \pm i \beta_{k} \mathrm{e}$ $\lambda_{ \pm k}^{n}=\alpha_{k}^{n} \pm i \beta_{k}^{n}$.

Página 119, linha 4, trocar $b_{+k}=1 / 2\left(a_{+k}^{n}+a_{-k}^{n}\right)$ e $b_{-k}=1 / 2\left(a_{+k}^{n}-a_{-k}^{n}\right)$ por $b_{+k}=$ $1 / 2\left(a_{+k}^{n}-i a_{-k}^{n}\right)$ e $b_{-k}=1 / 2\left(a_{+k}^{n}+i a_{-k}^{n}\right)$.

Página 132 , linhas -2 e -3 , trocar $K$ por $k$.

Página 133, linha 13, trocar $A_{\eta, n}^{\prime}\left(U_{n}\right)$ por $C_{\eta, n}^{\prime}\left(U_{n}\right)$.

Página 134, linha -3 , trocar $\bar{W}$ e $\bar{Z}$ por $W$ e $Z$.

Página 136, linha 6, trocar $\leq K^{2} K^{\prime 2} \frac{1}{\epsilon} \sum_{j=1}^{n}\left(\left\|C_{j+} \psi_{j+}^{n}+\right\| C_{j-}\left(\psi_{j-}^{n}\right)^{2}=K^{2} K^{\prime 2} \frac{1}{\epsilon}\|(W, Z)\|\right.$ por $\leq K^{2} K^{\prime 2} \frac{1}{\epsilon} \sum_{j=1}^{n}\left\|C_{j+} \psi_{j+}^{n}+C_{j-}\left(\psi_{j-}^{n}\right)\right\|^{2}=K^{2} K^{\prime 2} \frac{1}{\epsilon}\|(W, Z)\|^{2}$.

Página 136, linha -7 , trocar $\|(W, Z)\|$ por $\|(W, Z)\|^{2}$.

OBS: Elaborada posteriormente a defesa 